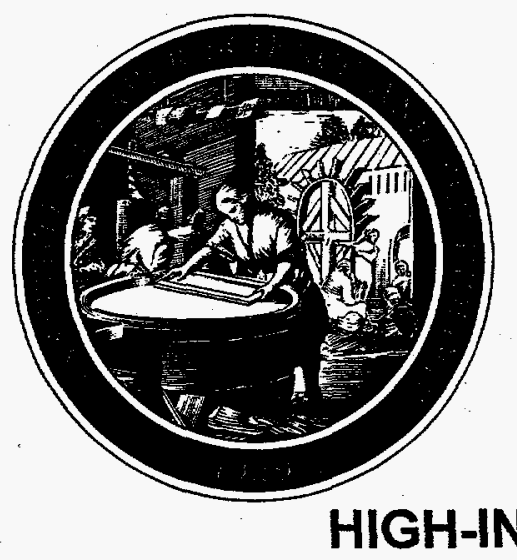

\title{
Institute of Paper Science and Technology
}

\section{HIGH-INTENSITY DRYING PROCESSES - IMPULSE DRYING}

PRODUCTION OF LINERBOARD ON A PILOT PAPER MACHINE, SUBSEQUENT COMMERCIAL CONVERTING TRIALS AND PRELIMINARY ECONOMIC ASSESSMENT

DOE/CE/40738-T15

Distribution Category UC-310

IPST Project 3595

REPORT 15

A Final Report

to the

U. S. DEPARTMENT OF ENERGY

April 1999

DISTABBUTON OF THIS DOCLNENT IS UARIMIED

\section{MASTER}
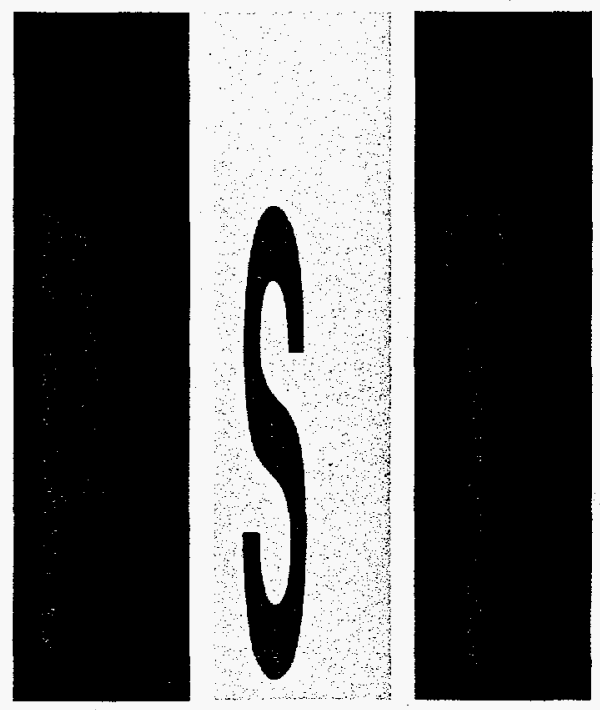


\section{INSTITUTE OF PAPER SCIENCE AND TECHNOLOGY PURPOSE AND MISSION STATEMENT}

The Institute of Paper Science and Technology is a unique organization whose charitable, educational, and scientific purpose evolves from the singular relationship between the Institute and the pulp and paper industry which has existed since 1929. The purpose of the Institute is fulfilled through three missions, which are:

- to provide high quality students with a multidisciplinary graduate educational experience which is of the highest standard of excellence recognized by the national academic community and which enables them to perform to their maximum potential in a society with a technological base; and

- to sustain an international position of leadership in dynamic scientific research which is participated in by both students and faculty and which is focused on areas of significance to the pulp and paper industry; and

- to contribute to the economic and technical well-being of the nation through innovative educational, informational, and technical services.

\section{ACCREDITATION}

The Institute of Paper Science and Technology is accredited by the Commission on Colleges of the Southern Association of Colleges and Schools to award the Master of Science and Doctor of Philosophy degrees.

\section{NOTICE AND DISCLAIMER}

The Institute of Paper Science and Technology (IPST) has provided a high standard of professional service and has put forth its best efforts within the time and funds available for this project. The information and conclusions are advisory and are intended only for internal use by any company who may receive this report. Each company must decide for itself the best approach to solving any problems it may have and how, or whether, this reported information should be considered in its approach.

IPST does not recommend particular products, procedures, materials, or service. These are included only in the interest of completeness within a laboratory context and budgetary constraint. Actual products, procedures, materials, and services used may differ and are peculiar to the operations of each company.

In no event shall IPST or its employees and agents have any obligation or liability for damages including, but not limited to, consequential damages arising out of or in connection with any company's use of or inability to use the reported information. IPST provides no warranty or guaranty of results.

The Institute of Paper Science and Technology assures equal opportunity to all qualified persons without regard to race, color, religion, sex, national origin, age, handicap, marital status, or Vietnam era veterans status in the admission to, participation in, treatment of, or employment in the programs and activities which the Institute operates. 


\section{DISCLAIMER}

This report was prepared as an account of work sponsored by an agency of the United States Government. Neither the United States Government nor any agency thereof, nor any of their employees, makes any warranty, express or implied, or assumes any legal liability or responsibility for the accuracy, completeness, or usefulness of any information, apparatus, product, or process disclosed, or represents that its use would not infringe privately owned rights. Reference herein to any specific commercial product, process, or service by trade name, trademark, manufac. turer, or otherwise does not necessarily constitute or imply its endorsement, recommendation, or favoring by the United States Government or any agency thereof. The views and opinions of authors expressed herein do not necessarily state or reflect those of the United States Government or any agency thereof. 


\section{DISCLAIMER}

Portions of this document may be illegible in electronic image products. Images are produced from the best available original document. 
DOE/CE/40738-T15

Distribution Category UC-310

\section{HIGH - INTENSITY DRYING PROCESSES - IMPULSE DRYING}

PRODUCTION OF LINERBOARD ON A PILOT PAPER MACHINE, SUBSEQUENT

COMMERCIAL CONVERTING TRIALS AND PRELIMINARY ECONOMIC ASSESSMENT

REPORT 15

A Final Report

To the

U.S. DEPARTMENT OF ENERGY

Work Performed Under Contract DE-FG07-85CE40738

By

D. I. Orloff

April 1999

INSTITUTE OF PAPER SCIENCE AND TECHNOLOGY

Atlanta, Georgia

Project 3595

Prepared for:

Valri Robinson

Program Manager

U.S. Department of Energy

Office of Industrial Programs

Conservation and Renewable Energy

Washington, DC 20585 
TABLE OF CONTENTS

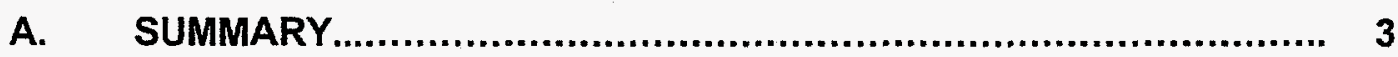

B. PILOT PRODUCTION TRIALS................................................ 4

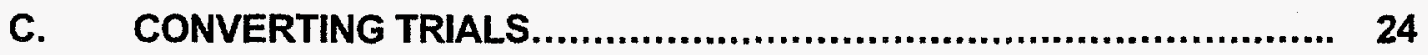

D. OPERATING ECONOMICS................................................ 43

E PAPER, BOARD AND BOX SAMPLING AND TESTING RESULTS........... 58 


\section{A. SUMMARY}

In September 1998, 33\# liner was produced on the \#4 pilot machine under both singlefelted wet pressing and impulse drying conditions. In October 1998, the pilot produced liner and commercial liner were converted to combined board and corrugated boxes at a commercial box plant. In January 1999, linerboard, medium, and combined board and box testing were completed.

The pilot trials demonstrated that $33 \#$ liner could be impulse dried at a reel speed of 380 $\mathrm{m} / \mathrm{min}$. Press dryness was improved by as much as 4 points, while CD STFI and CD ring crush were improved by more than $10 \%$. Improvements to the smoothness of heated side of sheet were also realized.

Commercial box plant converting trials demonstrated that impulse dried linerboard can be used to increase ECT and box compression strength by as much as $10 \%$. As anticipated, print quality was found to be superior.

A preliminary economic analysis was performed in which an impulse dryer would be installed on a dryer limited machine. It was assumed that the impulse dryer would increase press dryness by 4 points and would allow the basis weight to be reduced by $10 \%$. The economic model showed that the 4 points in dryness would translate to a $17 \%$ tonnage increase. Applying the $10 \%$ basis weight reduction resulted in an increase in productivity, on an area basis, of $30 \%$. The pulp cost savings was found to outweigh any additional electric power costs. 


\section{B. PILOT PRODUCTION TRIALS}

\section{B1.) Summary:}

In September 1998, the Institute and Beloit Corporation were successful in impulse drying $161 \mathrm{~g} / \mathrm{m}^{2}$ (33\#) linerboard on a one-meter wide pilot paper machine. This was the first time that impulse-dried linerboard had been reeled. The demonstration included a comparison of impulse drying to single-felted wet pressing.

Test results show that impulse drying, when compared to single-felted wet pressing, yields significant increases in press dryness, CD STFI, CD ring crush, and Mullen burst. The impulse-dried liner was also considerably smoother than the wet-pressed controls.

The demonstration also showed that runnability issues, such as start-up procedures, as well as operational issues, such as roll sticking and sheet delamination, have been resolved.

\section{B2.) Background}

Impulse drying has the promise of reducing capital costs, increasing machine productivity, reducing fiber use, reducing energy use, and improving paper physical properties. The Institute of Paper Science and Technology has been working to commercialize the impulse drying of board grades since the mid-1980s. In the early 1990s, the research focus was to control the physical aspects of the web to make it less susceptible to delamination, or to modify press roll surface properties to control heat flux. More recently $[1,2,3]$, work has been undertaken to control the cause of delamination, i.e., flash vaporization.

In a unique experiment [1], Institute researchers showed that application of increased ambient pressure during and after the nip opening process inhibits sheet delamination. This result had significant implications for impulse drying commercialization. The work suggested that by sufficiently increasing the ambient pressure at nip opening, press roll surface temperature could be increased without inducing web delamination. In a general way, the work pointed out the importance of properly designing and controlling the nip opening process.

In subsequent research [2], temperature distributions were measured within layers of impulse-dried sheets during nip opening to various ambient pressures. Using these data and thermodynamic reasoning, pressure profiles were determined within the web.

Based on these profiles, the hypothesis that delamination was caused by an imbalance of internal and external sheet pressure was tested. The results supported the view that delamination occurs when the pressure difference across the sheet is too high, and the buildup of internal pressure disrupts the sheet.

While opening the nip to ambient pressures in excess of one atmosphere may eventually prove to be practical, other methods that may be easier to implement were sought. In particular, subsequent laboratory experiments [3] showed that delamination could be inhibited by properly controlling the load applied to the sheet as the nip opens. The experiment consisted of identifying nip opening load conditions that would be sufficient to suppress the delamination of linerboard handsheets. The work demonstrated that delamination could be inhibited by applying a controlled decompression during nip opening. 
Utilizing these discoveries, the Institute of Paper Science and Technology and the Beloit Corporation embarked on a joint project to develop impulse drying for application to board grades. The objectives of the project were to develop the necessary technology, to demonstrate the technology on a pilot paper machine, and to conduct converting trials at a commercial box plant.

In a recent paper [4], Institute and Beloit personnel described the process modifications that allow impulse drying of board grades to become commercially feasible and reported the results of initial pilot paper-machine experiments.

These initial pilot paper-machine experiments confirmed that the ramp decompression concept could be used to increase critical impulse drying temperature, thus opening the operating window of the technology. A specially designed adjustable ramp shoe allowed on-the-fly adjustment of nip decompression, which facilitated optimization. In addition, a combination of a specially designed press roll surface and the use of a TET doctor helped to eliminate picking and control sticking. Venting of the nip and blanket groove geometry were also found to be important.

\section{B3.) Experimental}

In September 1998, success was achieved in producing 33\# linerboard on Beloit's \#4 pilot paper machine. This is the first time that impulse dried linerboard had been reeled. The demonstration included a comparison of impulse drying to single-felted wet pressing. Figure B1 shows a schematic diagram of the press section of the pilot paper machine. The machine consisted of a gap former, bi-nip press, a shoe press, a dryer section, calender, and reel. The shoe press was of a closed design that could function as a single-felted wet press or as an impulse dryer. The shoe press was outfitted with a 0.23 -m-long standard shoe followed by a $0.11-\mathrm{m}$-long ramp shoe. The pressure profile of the ramp shoe could be adjusted "on-the-fly" until the ramp profile was optimized to achieve the highest press roll temperature without experiencing sheet delamination. Figure B2 shows the ramp shoe pressure profile centered in the $C D$ direction and measured from the position of peak load pressure. The profile is similar to that used in previously reported experiments on Beloit's No. 2 pilot paper machine [4].

Table B1 shows the chronology of the linerboard production trials. Reels of impulse-dried liner were produced at two press roll temperatures and two calender loadings on the first day. Press dryness measurements were also taken. On the second day, reels of single-felted wet-pressed liner at two calender loadings were produced. Measurements of press dryness were taken and calendering experiments were conducted to determine the impact of calender loading on linerboard properties. The second day was also used to repeat, over a range of press roll temperatures, the impulse drying that was accomplished on the first day.

Table B2 shows the paper-machine conditions that were recorded for the two days of the trial. Freeness was targeted at $650 \mathrm{ml} \mathrm{CSF}$ for both days. Note that the freeness during the first day was $613 \mathrm{ml} \mathrm{CSF}$ and during the second day was $669 \mathrm{ml} \mathrm{CSF}$. This difference in refining level was inadvertent and was only discovered at the end of the second day of trials.

Table B3 shows web solids as measured after the couch, after the flatbox, and after the bi-nip press that was ahead of the impulse dryer. 
Detailed measurements of the physical properties of the impulse-dried linerboard made at various press roll surface temperatures on the first and second day showed that they were slightly different. These differences are explored in the physical property development section of this paper.

\section{B4.) Economics of Energy Usage}

The electric power usage of the induction heating system was measured during the impulse drying experiments on both days. On the first day, while the reels were being produced, the induction heating system drew $495 \mathrm{~kW}$ at a roll temperature of $255^{\circ} \mathrm{C}$ and $531 \mathrm{~kW}$ at a roll temperature of $271^{\circ} \mathrm{C}$.

Figure B3 shows the electric power usage as a function of average press roll surface temperature as measured on both days of the linerboard trials. Note that the energy usage on Day \#1, during the reel production phase of the trial, was lower than on Day \#2, when short duration experiments were conducted at increasing temperature. Note also that the later data are less consistent. This comparison suggests that the press roll was not in equilibrium during the later experiments.

Based on electric power usage data from Day \#1, $171.5 \mathrm{~kW}$-hr/ton were used when the roll was heated to a target roll temperature of $260^{\circ} \mathrm{C}$. Based on an estimated electrical power cost of $\$ 0.03 / \mathrm{kW}-\mathrm{hr}$, our roll heating cost was $\$ 5.14 / \mathrm{ton}$. The estimated cost savings in reduced steam usage (assuming a 3.8-point increase in dryness at the press section and a $\$ 2.83$ million Btu steam cost) was $\$ 1.18 /$ ton. Since some of the improvement in physical properties was due to increased refining, the estimated electric power costs associated with this incremental refining was $\$ 0.79 /$ ton (based on an estimate of $26.3 \mathrm{~kW}$-hr/ton to refine from $669 \mathrm{ml} \mathrm{CSF}$ to $613 \mathrm{ml} \mathrm{CSF}$ ). Hence, the net increase in energy costs was about $\$ 4.75 /$ ton. Therefore, to make this application viable, there must be fiber savings and productivity improvements that justify a $\$ 4.75 /$ ton energy cost penalty.

\section{B5.) Press Solids}

Figure B4 shows press solids outgoing from the impulse dryer as a function of target roll surface temperature for experiments performed on the first and second day of the trials. Also included are the outgoing press solids for the wet pressing performed on Day \#2 of the trials. As will be shown in the physical property development section, impulse drying temperatures of as high as $260^{\circ} \mathrm{C}$ could be reached without the sheet showing signs of sheet delamination. Hence, impulse drying could be used to increase outgoing solids by about 3.3 to 4.0 points of dryness as compared to the wet-pressed control.

\section{B6.) Physical Property Development}

\section{Preliminary Measurements}

Linerboard properties were measured at Beloit's paper testing laboratory. In these measurements, there was no attempt to distinguish cross-directional variations in paper . physical properties. In addition, physical property indexes are based on average conditioned basis weight and no. confidence limits were available. Based on the reported results, Figures B5 and B6 show CD STFI compression index and CD ring crush index, respectively, plotted against target roll surface temperature. Comparing impulse drying 
(from the first day of the trial) to single-felted wet pressing (from the second day of the trial), there is [7] an $18 \%$ improvement in CD STFI and a $7 \%$ improvement in CD ring crush.

\section{Finalized Measurements}

Detailed measurement of linerboard properties was undertaken at the Institute of Paper Science and Technology. In these measurements, the linerboard was tested in three cross-directional lanes (operator lane, center, and drive lane). Test frequency was increased so that it would reduce the error bars $(95 \%$ confidence limits) to an acceptable level. In addition, physical property indexes are based on oven-dried weights of individual test strips.

The cross-directional profile of the reels of linerboard produced on the first and second day of the trial was explored. Figure B7 shows CD SFTI index and Figure B8 shows MD STFI index, both as measured in the drive, center, and operator lanes of the single-felted wet-pressed and impulse-dried linerboard. The drive lane is presented as a white bar, the center lane is reported as a black bar, and the operator lane is shown as a gray bar. In Figure B7 the operator lane was normally stronger than the drive lane, which was in turn stronger than the center lane. In Figure B8 the operator lane was stronger than the center lane, which was stronger than the drive lane. This could be an artifact of cross directional nonuniformities (pressure, moisture, fiber orientation, and basis weight) associated with the setup of the paper machine. Figure B9 shows the MD/CD tensile ratio as measured in each of the three lanes. The web was consistently MD oriented (with an MD/CD tensile ratio of about 2.5) and tended to be more MD oriented in the center lane. The fact that CD STFI index tended to be lowest in the center lane suggests the need to also measure properties of corrugated board in edge and center lanes.

The data has been averaged over the web width in the remaining figures showing linerboard properties. In previous work, it was found that use of zd-ultrasonics is an effective and sensitive test for sheet delamination. Figure B10 shows the zd-specific elastic modulus of wet-pressed and impulse-dried linerboard as a function of the target roll surface temperature. Note that there was a drop-off in modulus at roll temperatures above $260^{\circ} \mathrm{C}$. This suggests a critical impulse drying temperature of $260^{\circ} \mathrm{C}$ for the experiments.

Figures B11 and B12 show the CD STFI index and CD ring crush index, respectively, as plotted against target roll surface temperature. Both of these properties increase with increased roll temperature. It is important to compare these properties in a range of roll temperatures from 240 to $260^{\circ} \mathrm{C}$ on both days of the trial. It is observed that the strength of the impulse-dried liner produced on the first day of the trial tended to be stronger than that produced at a similar temperature on the second day. This can also be seen in Figure $B 13$, where CD STFI index is plotted as a function of apparent density. Hence, the difference is attributed to increased refining on the first day.

While CD STFI compression strength and CD ring crush influence the ultimate strength of corrugated board, linerboard smoothness is most important as a predictor of printability [8]. In the present work, the smoothness of the roll side of the linerboard was measured as Bendtsen roughness and as Emveco roughness. Figure B14 reports the micro average Emveco roughness of the hot roll side of liner produced during our trials. Figure $B 15$ reports the micro deviation Emveco roughness. In both cases the roughness in both the $C D$ and MD were recorded. It is observed that the samples are always smoother in the MD. It is also observed that the samples become smoother as the roll surface temperature was increased and when the liner is calendered. The key finding is that 
impulse drying significantly reduced the roughness of the linerboard and that impulsedried liner would not need to be calendered.

In summary, Table B4 shows the percent improvement in critical physical properties of the reels of impulse dried linerboard as compared to the appropriate wet-pressed controls. Impulse drying was found to increase; CD STFI by about $10 \%$, CD ring crush by between 11 to $14 \%$, and Mullen burst by between 13 and $20 \%$. Hence, basis weight reductions of $10 \%$ or more may be possible.

\section{B7.) References}

1. Orloff, D. I., Patterson, T., Krause, A. M., "Opening the Operating Window of Impulse Drying - Part I. The Effect of Ambient Pressure at Nip Opening," Tappi Journal 81(7):113116 (July 1998).

2. Orloff, D. I., Patterson, T., Parviainen, P. M., "Opening the Operating Window of Impulse Drying - Part II. Pressure Differential as a Source Of Delamination," Tappi Journal 81(8):195-203 (August 1998).

3. Orloff, D. I., Patterson, T., Phelan, P., Rudman, I., "Opening the Operating Window of Impulse Drying - Part III. Controlled Decompression Experiments," Tappi Joumal 81(9):159-162 (September 1998).

4. Orloff, D. I. and Crouse, J. W., "Impulse Drying: Status of the Pilot-Scale Research Program," 1998 TAPPI Proceedings of the International Engineering Conference, Book 3, pp. 879-902, September 13-17, 1998.

5. Orloff, D.I., and Sobczynski, S.F., "Impulse Drying Pilot Press Demonstration: Ceramic Surfaces Inhibit Delamination," Paper Technology 34(10): 24-33 (December 1993).

6. Soczka, E., personal communication, December 1998, Institute of Paper Science and Technology, Atlanta, Georgia.

7. Orloff, D. I., "Impulse Drying of Board Grades: An Emerging Technology," PaperAge, 114(12): 22-23 (December 1998).

8. Orloff, D.I., Challas, J., and Rudman, I., "Flexographic Printing of Linerboard," TAGA Proceedings, 1:277-303(1995).

\section{B8.) Acknowledgements}

The author would like to thank the Member Companies of the Institute of Paper Science and Technology, the Beloit Corporation, and the U.S. Department of Energy (through Grant No. DOE/CE/40738) for supporting this research. The author would also like to thank Jere Crouse and David Lange of Beloit Corporation and Charles McWilliams of Tekscan, iric. for their contributions to this work. 
B9.) Tables

Table B1. Chronology of Linerboard Production Trials

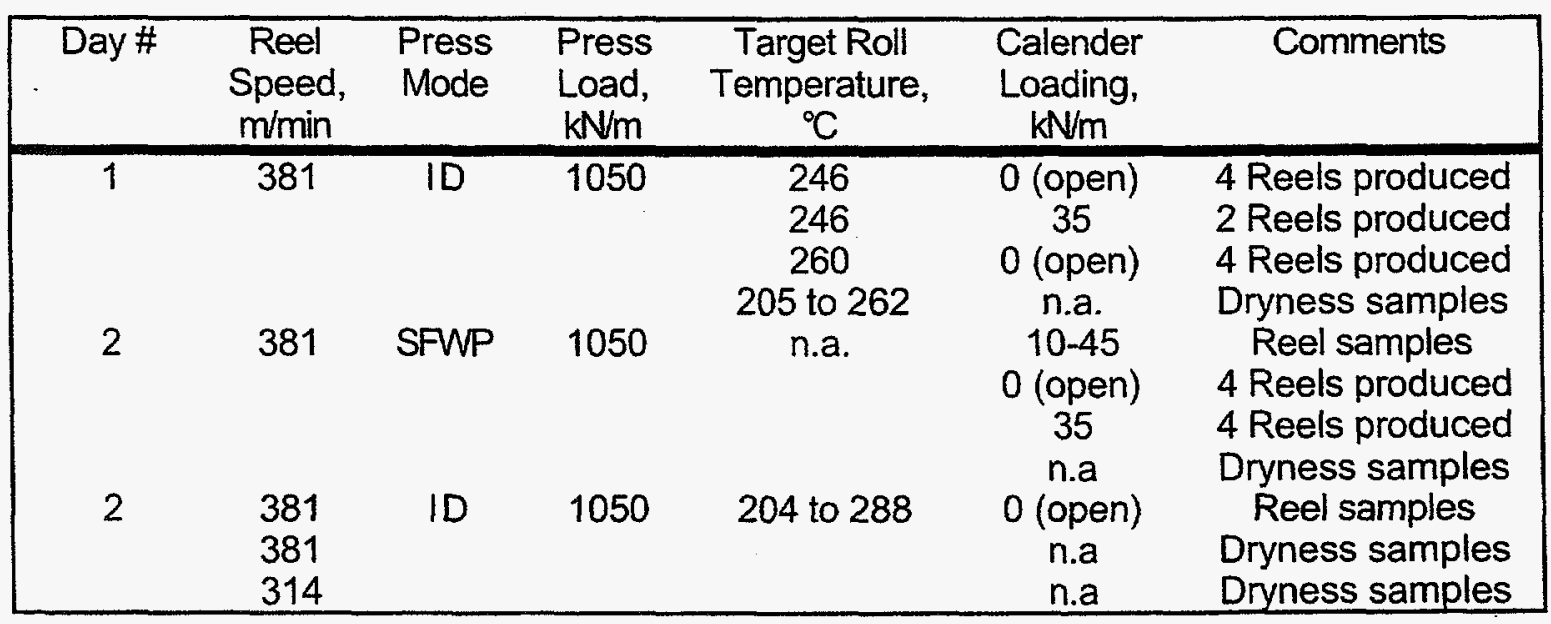

Table B2. Typical Production Conditions

\begin{tabular}{|c|c|c|}
\hline Condition & Day 1 & Day 2 \\
\hline Machine Chest Temp., ${ }^{\circ} \mathrm{C}$ & 58 & 63 \\
\hline Freeness, ml CSF & 613 & 669 \\
\hline WRV & 2.15 & 2.05 \\
\hline Target Cond. Basis Wt, gsm & 160 & 160 \\
\hline Jet-to-Wire Ratio & 1.22 & 1.22 \\
\hline 1st Press Load, kN/m & 105 & 105 \\
\hline 2nd Press Load, kN/m & 140 & 140 \\
\hline Calender Temp., ${ }^{\circ} \mathrm{C}$ & 121 & 121 \\
\hline Target Reel Moisture, $\%$ & 5 & 5 \\
\hline
\end{tabular}

Table B3. Typical Press Solids

\begin{tabular}{|c|c|c|}
\hline Condition & Day 1 & Day 2 \\
\hline After Couch, \% Solids & 20.2 & 21.0 \\
\hline After Flatbox, \% Solids & 23.5 & 24.5 \\
\hline After Bi-Nip, \% Solids & 40.4 & 42.0 \\
\hline
\end{tabular}


Table B4. Percentage Improvement in Linerboard Properties (Compared to the Wet Pressed Controls)

\begin{tabular}{|c|c|c|c|c|}
\hline $\begin{array}{c}\text { Impulse-Drying } \\
\text { Temperature, } \\
{ }^{\circ} \mathrm{C}\end{array}$ & Calendering & $\begin{array}{c}\text { Improvement } \\
\text { CD STFI, } \\
\%\end{array}$ & $\begin{array}{c}\text { Improvement } \\
\text { CD Ring Crush, } \\
\%\end{array}$ & $\begin{array}{c}\text { Improvement } \\
\text { Mullen Burst, } \\
\%\end{array}$ \\
\hline 246 & no & 9.8 & 11.4 & 17.1 \\
\hline 260 & no & 9.7 & 11.4 & 13.2 \\
\hline 246 & yes & 9.4 & 13.7 & 19.8 \\
\hline
\end{tabular}

\section{B10.) Figures}

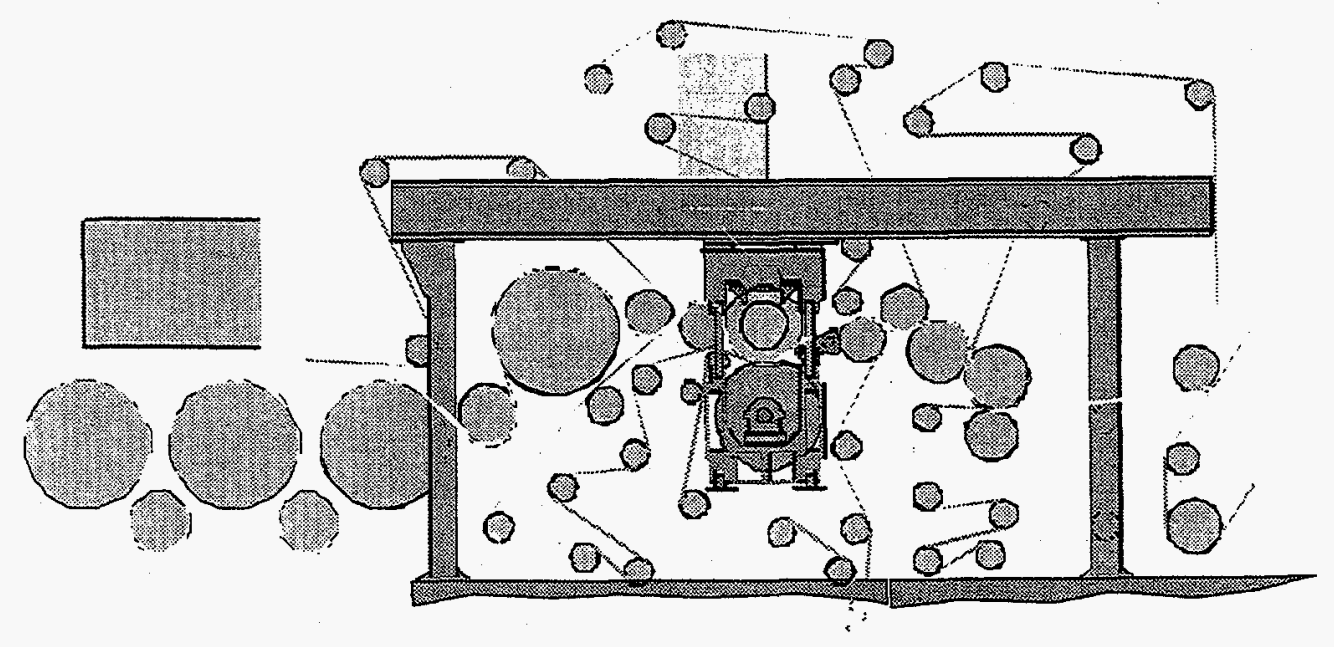

Figure B1. Press Section of the Pilot Paper Machine Showing the Impuise Dryer. 


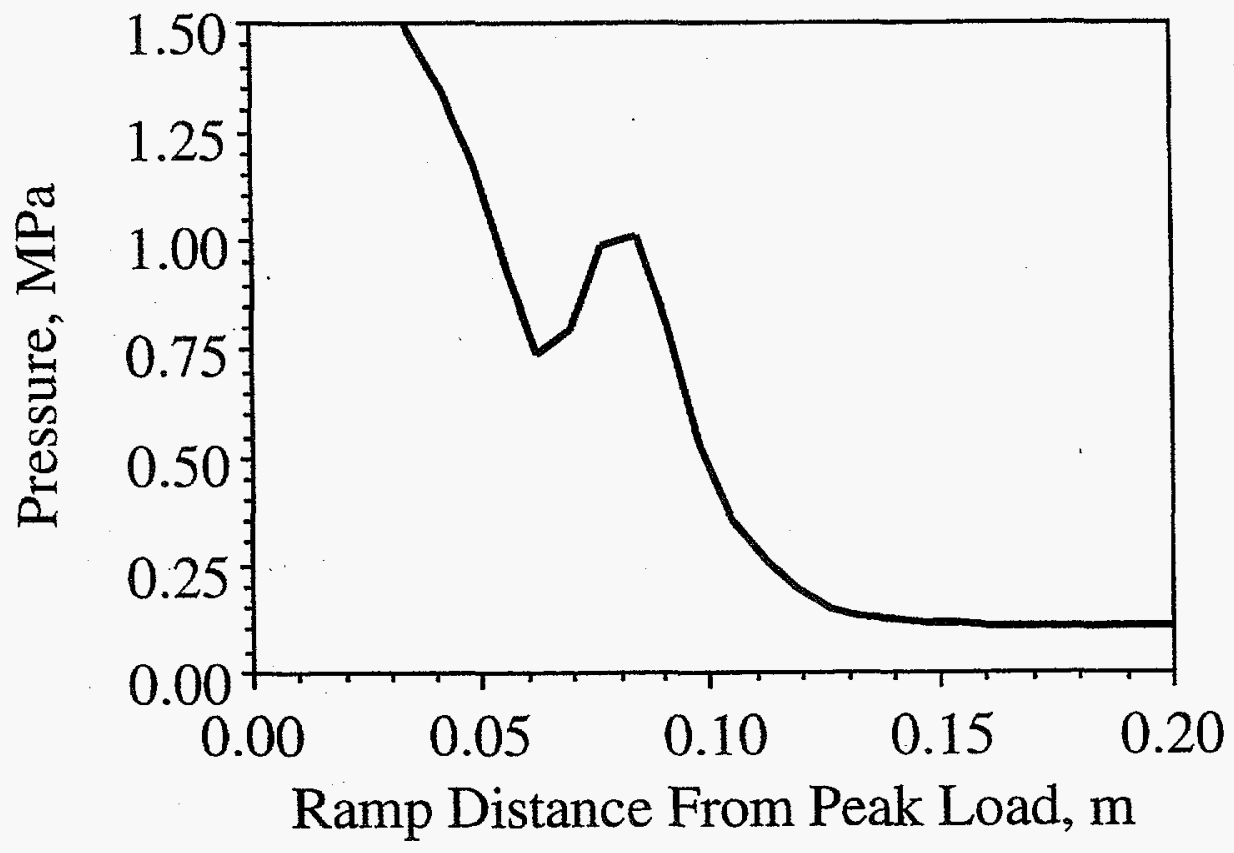

Figure B2. Ramp Pressure Profile. 


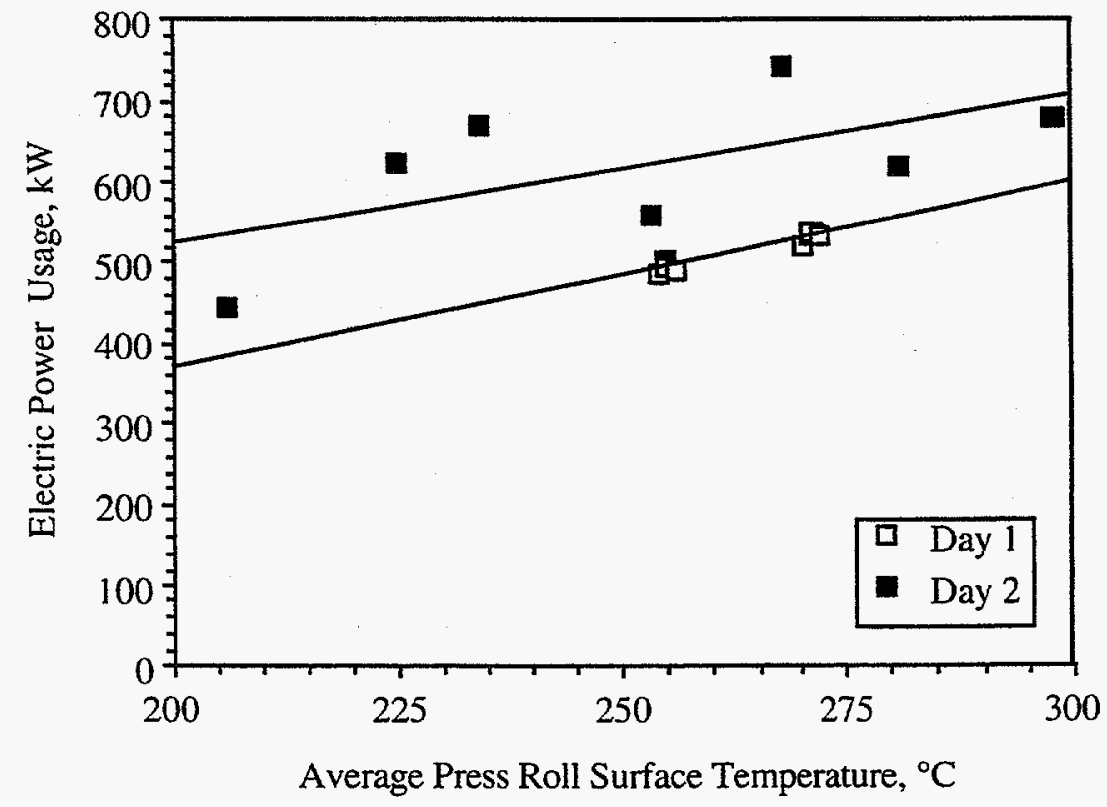

Figure B3. Electric Power Usage versus Average Press Roll Temperature. 


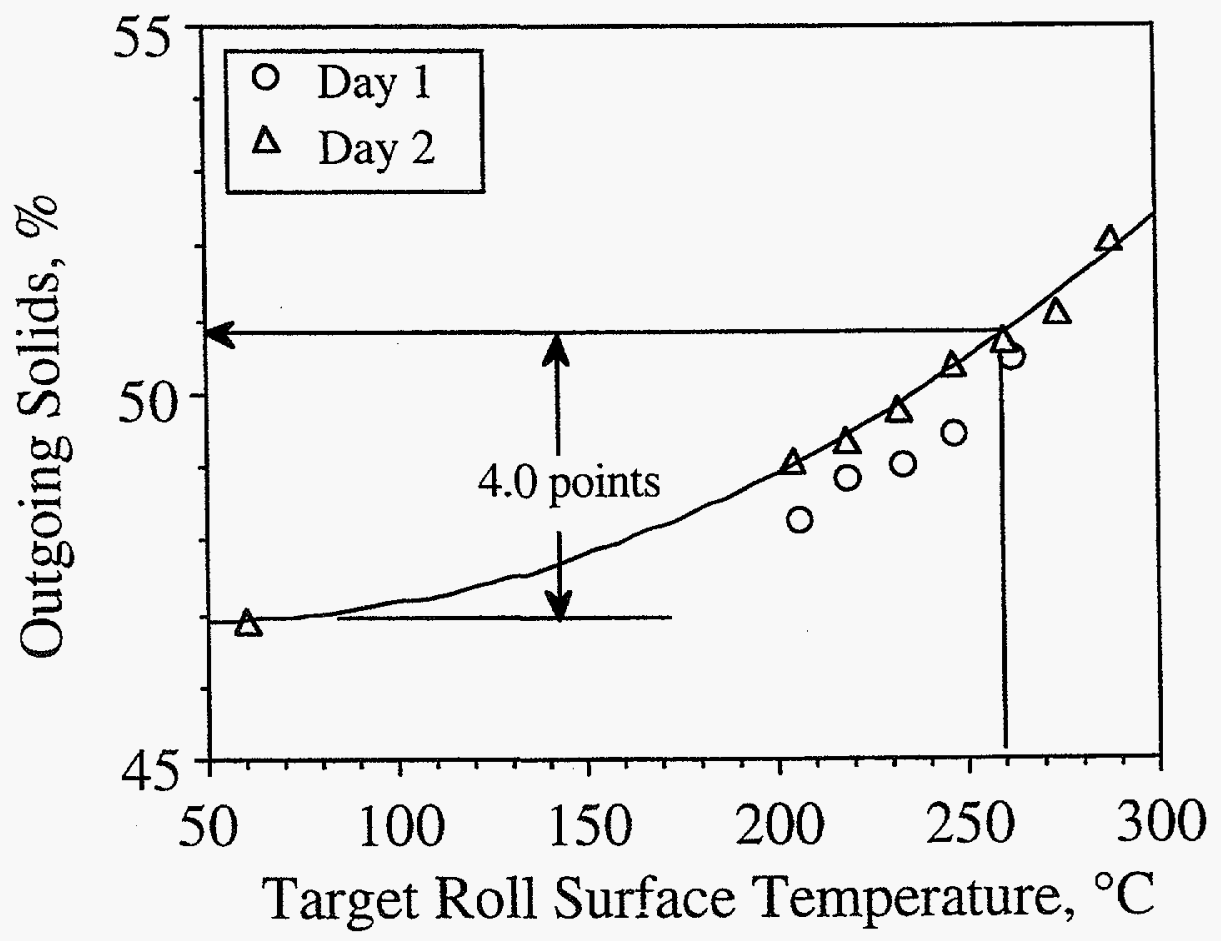

Figure B4. Outgoing Solids versus Target Roll Surface Temperature. 


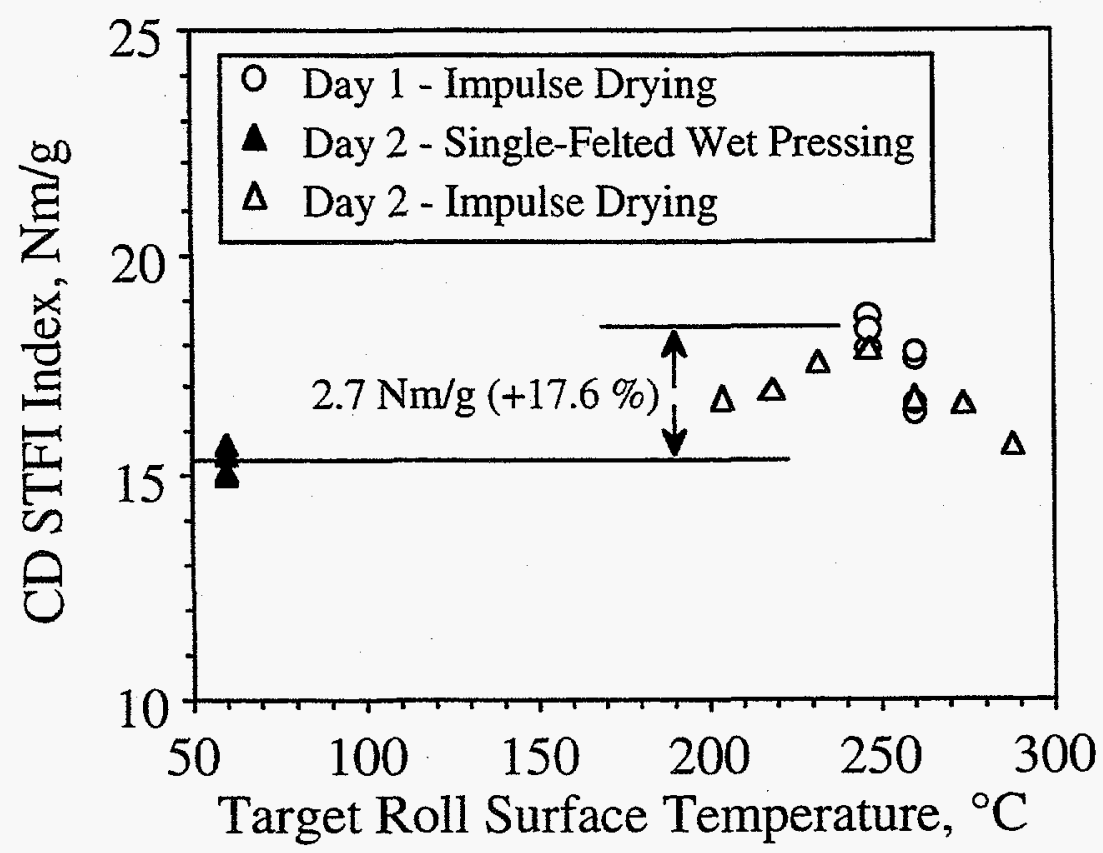

Figure B5. Cross-Directional STFI Compression Strength Index versus Target Roll Surface Temperature. 


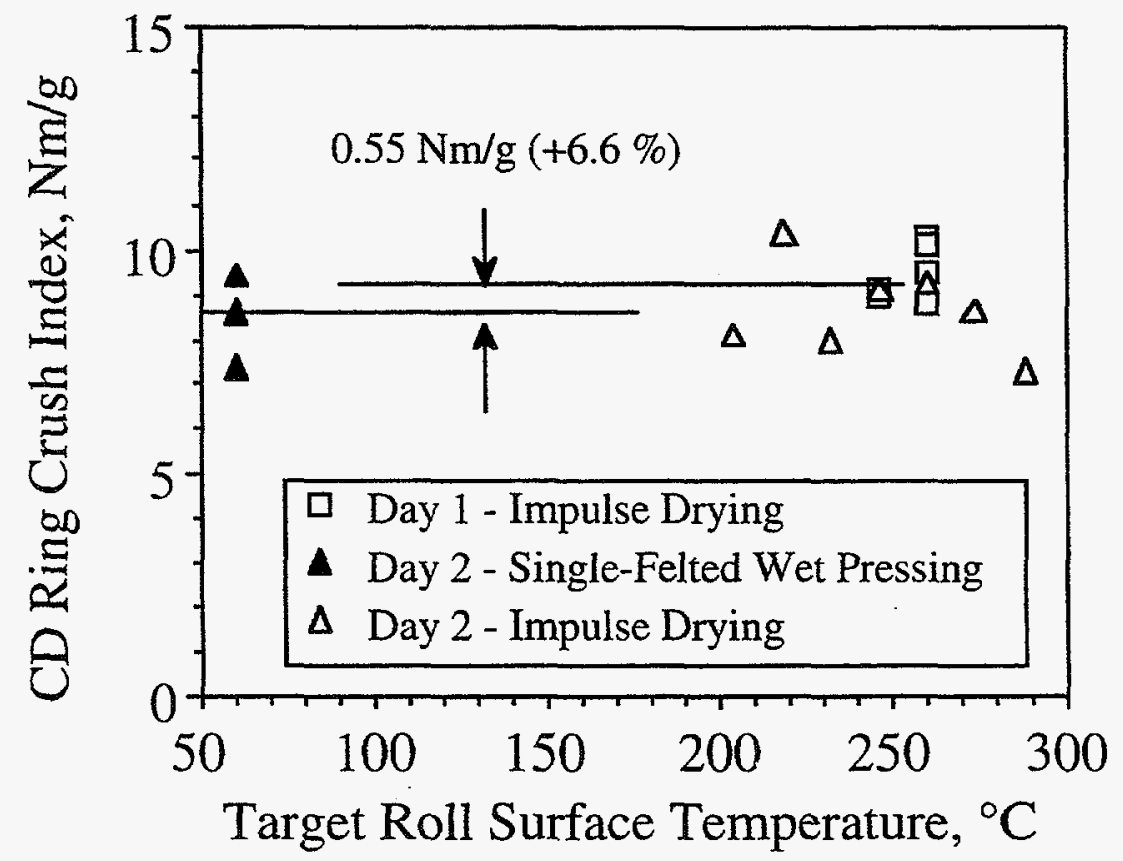

Figure B6. Cross-Directional Ring Crush Index versus Target Roll Surface Temperature. 


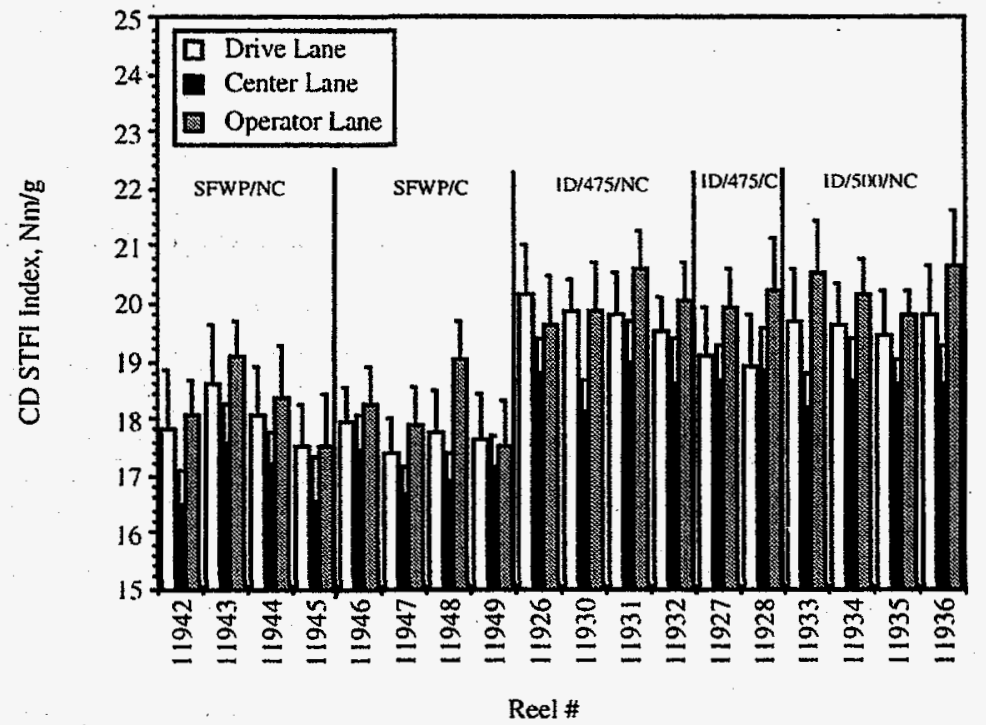

Figure B7. Cross-Directional STFI Index versus Reel Number 


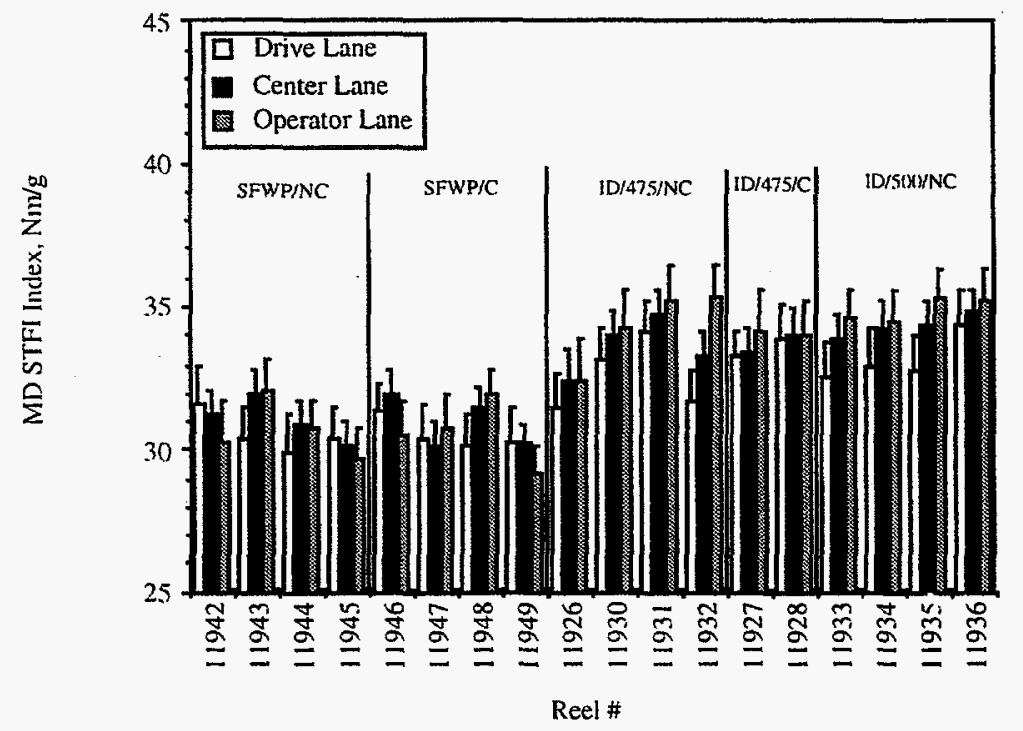

Figure B8. Machine-Directional STFI Index versus Reel Number 


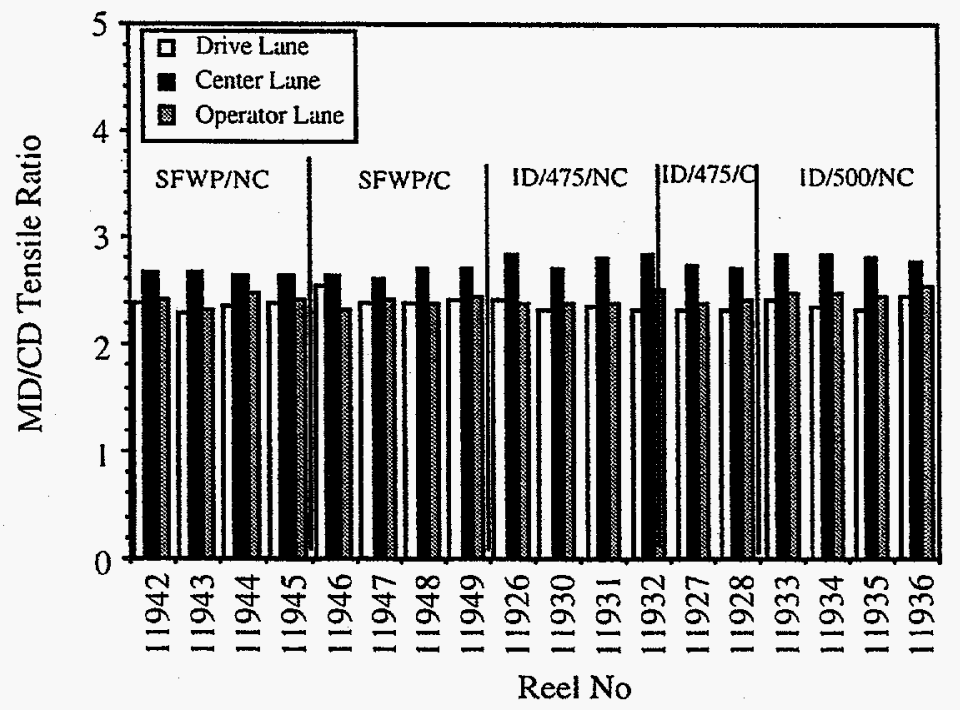

Figure B9. MD/CD Tensile Ratio Versus Reel Number 


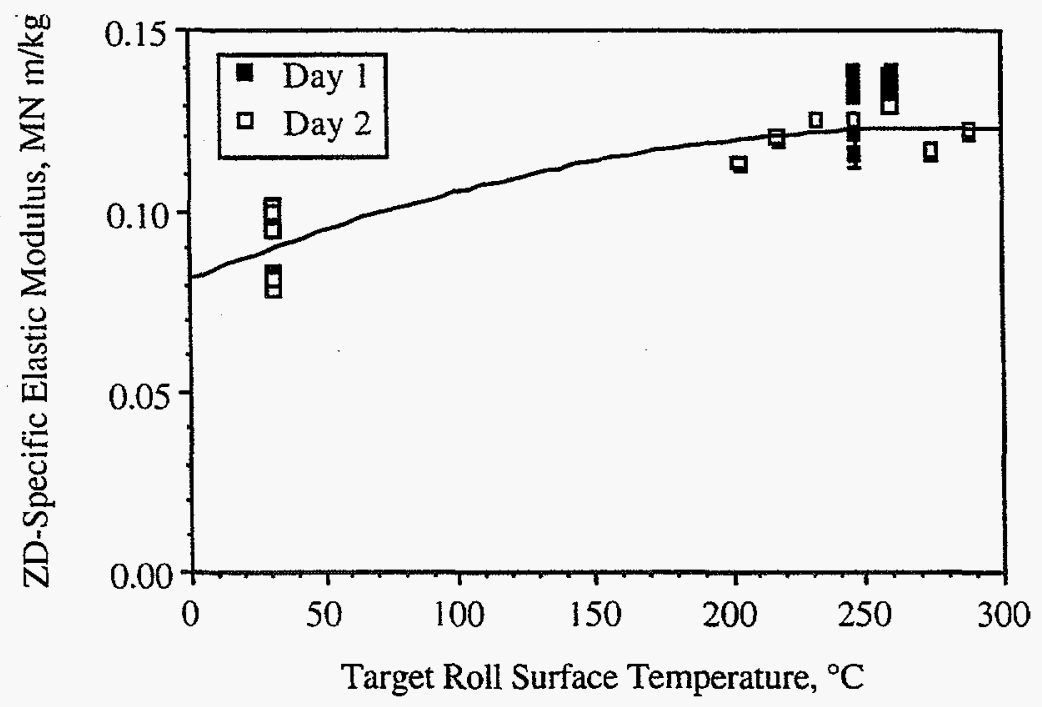

Figure B10. ZD- Specific Elastic Modulus versus Target Roll Surface Temperature. 


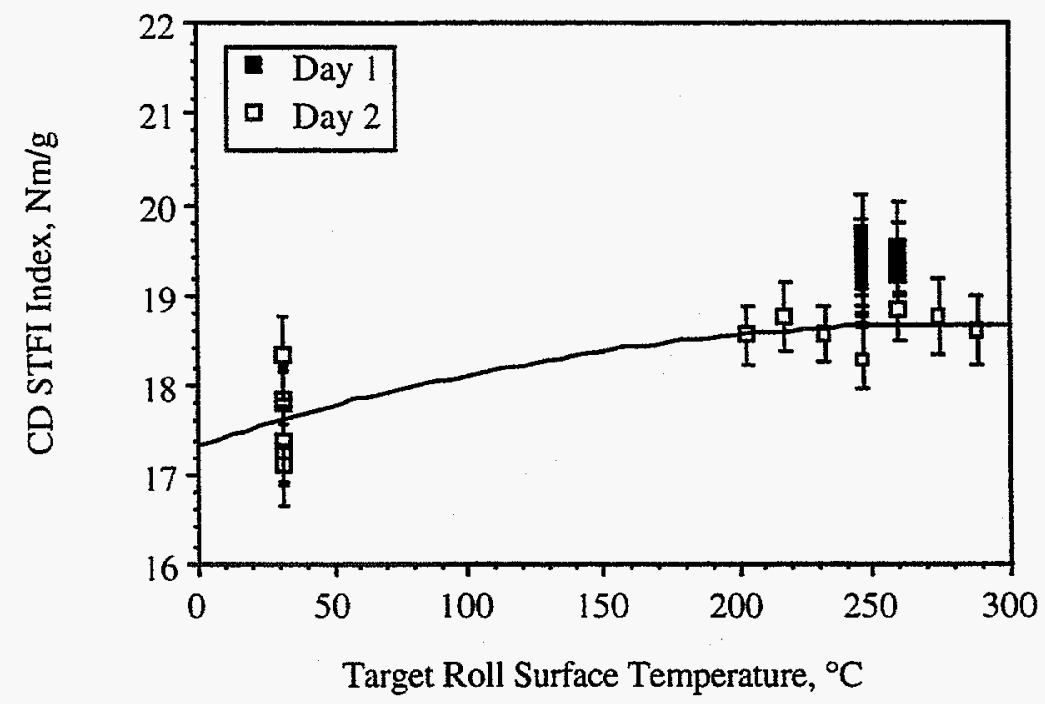

Figure B11. Cross-Directional STFI Compression Index versus Target Roll Surface Temperature. 


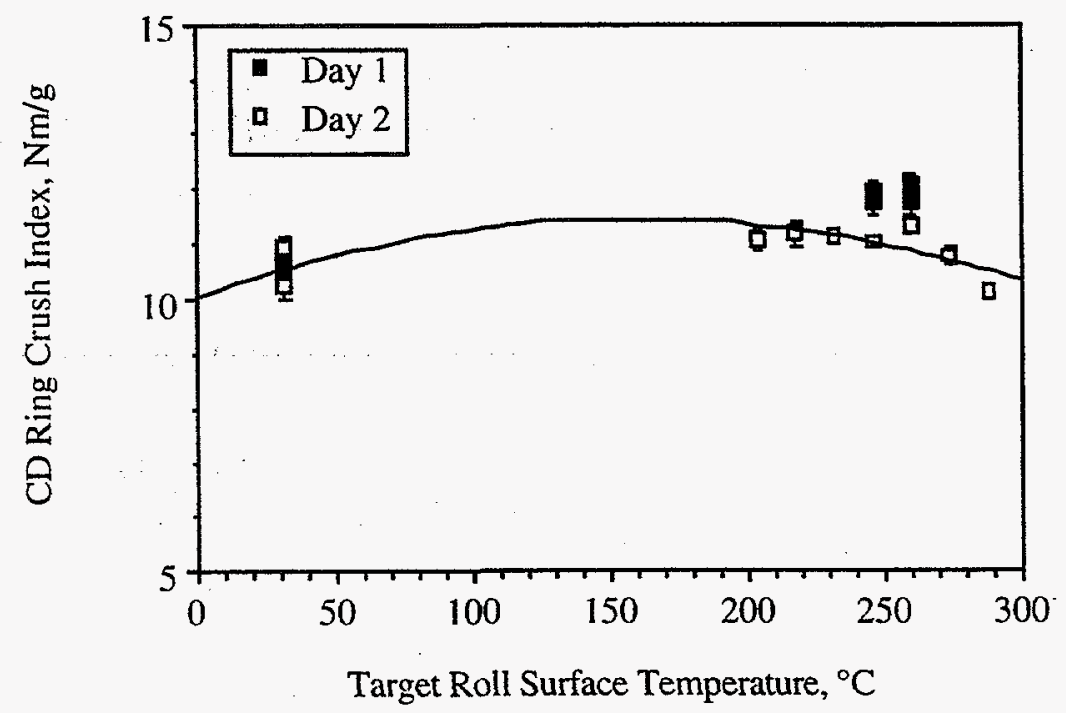

Figure B12. Cross-Directional Ring Crush Index versus Target Roll Surface Temperature. 


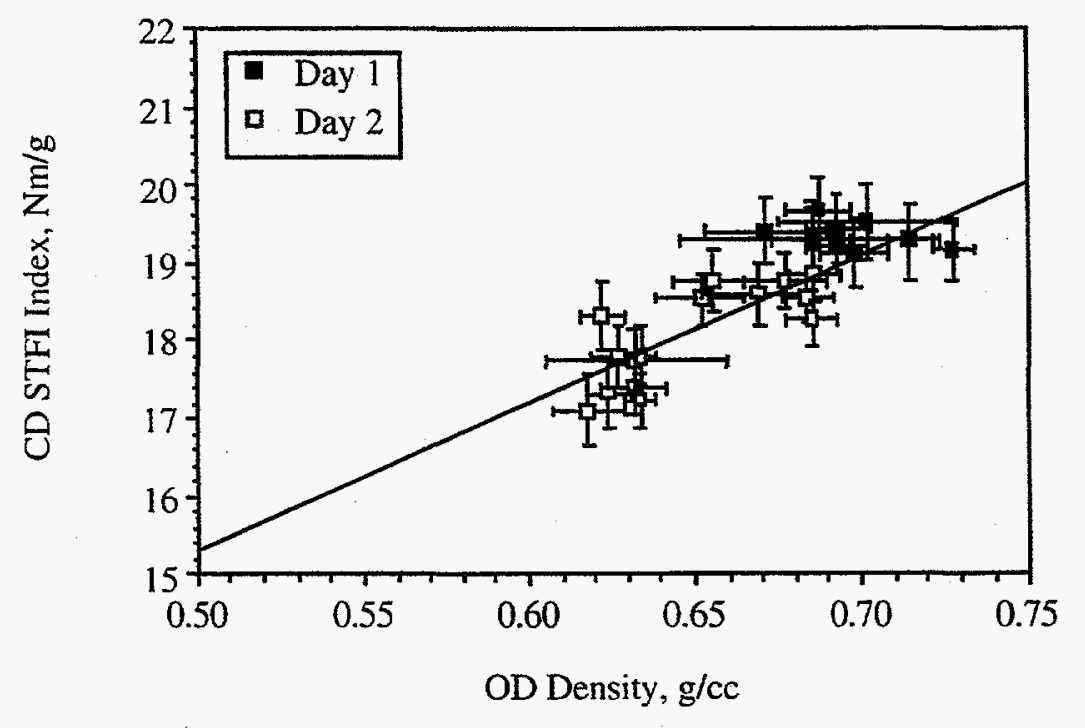

Figure B13. Cross-Directional STFI Compression index versus OD Density. 
Roll Side Emveco Roughness, Micro Average

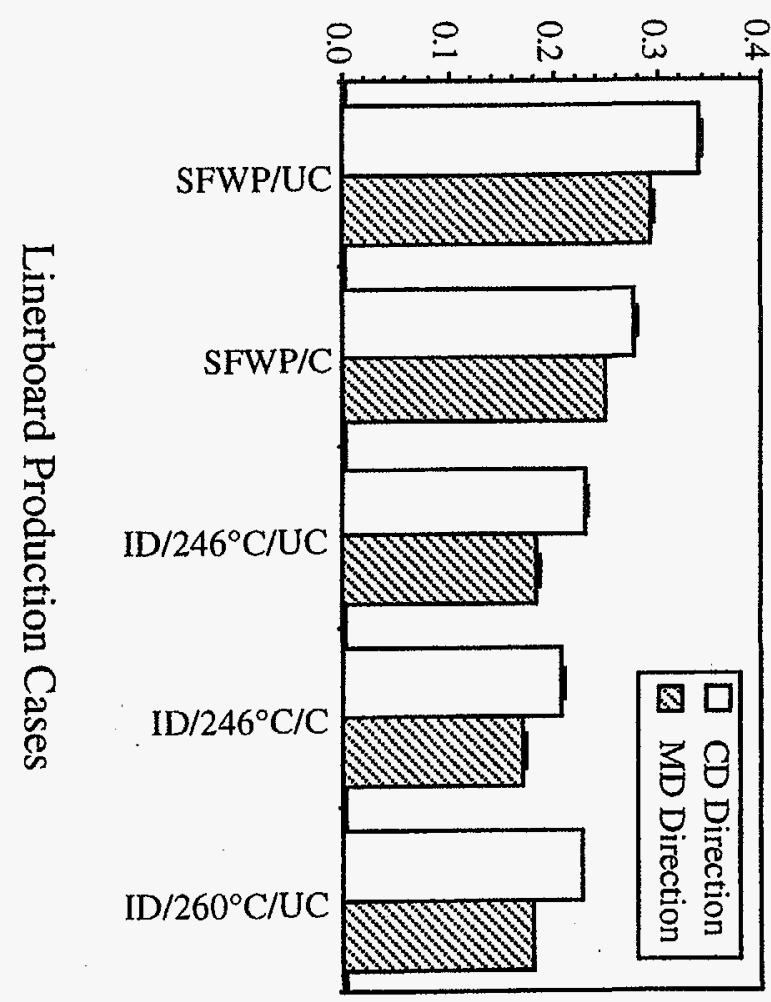




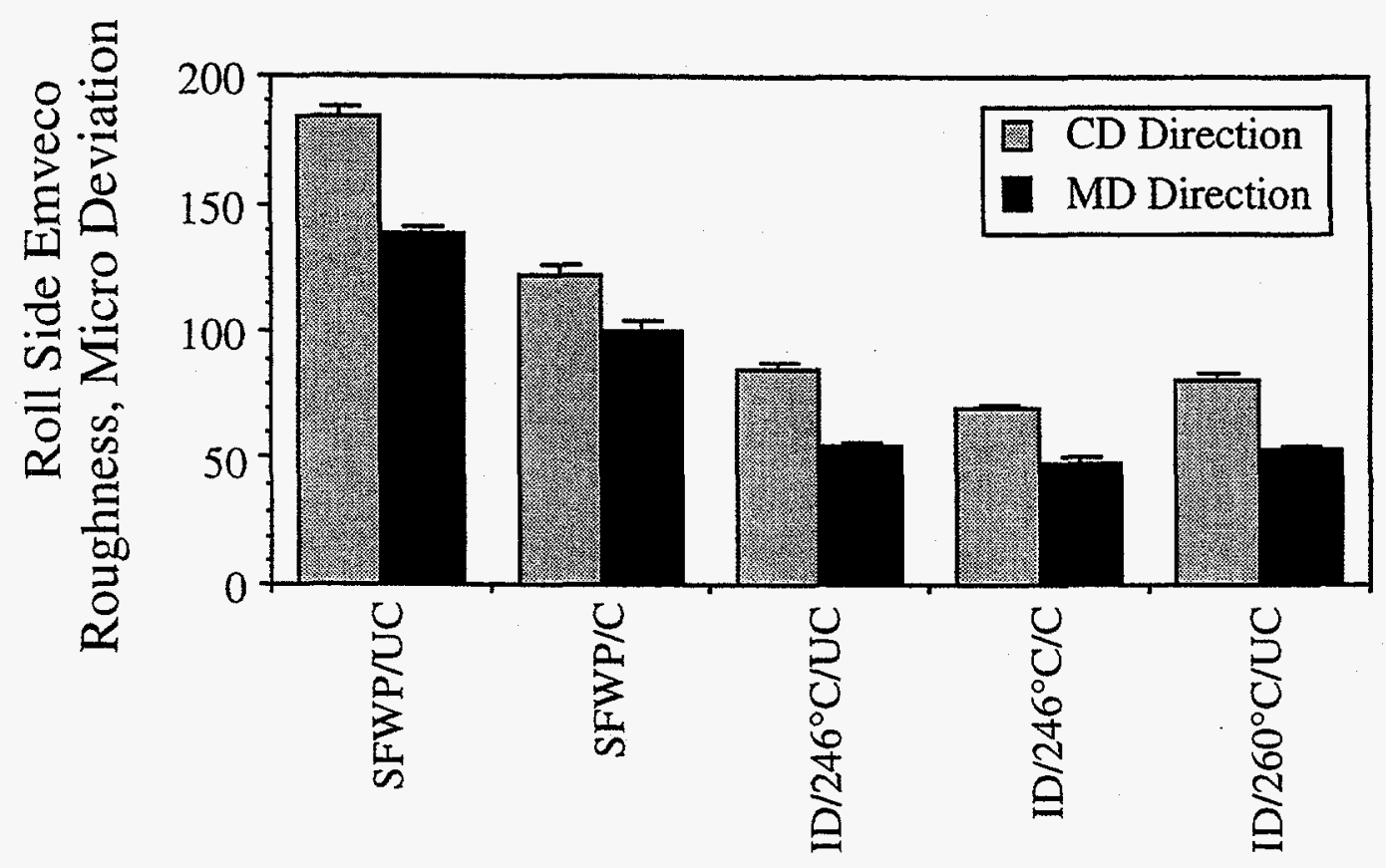

\section{Linerboard Production Cases}

Figure B15. Micro Deviation Roll Side Emveco Roughness for Linerboard Production Cases.

\section{CONVERTING TRIALS}

\section{C1.) Summary}

Reels of linerboard produced on a pilot paper machine were converted on a commercial corrugator and the results compared to those obtained for a commercial liner. The pilot paper machine-produced liner included single-felted wet pressed liner as well as impulse dried liner.

The converting trials were conducted at the Stone Container plant in Keokuk, lowa, in October 1998. The flexo, folder, gluer operation proceeded quite smoothly. In addition, there were no problems encountered during die cutting and no score cracking problems were noted. Finished containers were tested at the Institute for edge crush, flexural stiffness, pin adhesion, and box compression strength.

The performance of the impulse dried liner was compared to the single-felted wet pressed control as well as the commercial sample. 


\section{C2.) Experimental}

The plan was to convert the Beloit $0.76 \mathrm{~m}$ (30 inch) wide rolls on a commercial corrugator to make combined board. Each run would produce a minimum of 1000 blanks, $1.52 \mathrm{~m}$ (60 inches) long and $0.69 \mathrm{~m}$ ( 27 inches) wide, from which 750 would be printed and converted into shipping containers and 250 blanks would be die cut and not printed. The resulting single-wall container would be $0.38 \mathrm{~m}$ (15 inches) long, $0.36 \mathrm{~m}$ (14 inches wide), and $0.30 \mathrm{~m}$ (12 inches) high.

A printing plate was selected for printing comparisons. The print copy included an Institute of Paper Science and Technology letterhead logo that was enlarged to $0.15 \mathrm{~m}$ (6 inches) in diameter. It also included conventional halftones of Edgar Allen Poe; lines, from $1 / 2$ point to 8 point; portions of UPC and shipper UPC; and a heliograph of a child car seat.

A number of container plants were contacted to determine if they could run five sets of narrow $0.76 \mathrm{~m}$ (30 inch) wide rolls to produce combined board, as well as print and produce containers from impulse dried $161 \mathrm{~g} / \mathrm{m}^{2}(33 \#) \mathrm{kraft}$ linerboard made on Beloit pilot paper machine. One set of $161 \mathrm{~g} / \mathrm{m}^{2}(33 \#)$ commercial kraft liner in any roll of their plant inventory would also be run. Each of the six runs would have a maximum of 3048 lineal meters $(10,000$ lineal feet). If the runs on the corrugator went smoothly, it could be shortened to 1524 lineal meters (5000 lineal feet).

The results from the inquiries were not encouraging at first; however, we eventually were fortunate to receive a positive response from the Stone Container plant in Keokuk, lowa. Converting trials were conducted in October 1998.

Tables $\mathrm{C} 1$ through $\mathrm{C} 3$ show specific details of the three unit operations performed at the box plant. The corrugator crew had been fully informed and coached by the plant manager in advance of the run and the implementation of the actual run went very smoothly. The single-facer and double-backer splicers were loaded with appropriate rolls in the planned sequence and when 305 lineal meters (1000 lineal feet) had been produced, the splicers were manually turned on starting the next sequence. The commercial rolls of $161 \mathrm{~g} / \mathrm{m}^{2}$ (33\#) liner were run as a wider roll, $1.689 \mathrm{~m}$ (66.5 inch), from the plant inventory. The corrugating medium, $1.686 \mathrm{~m}$ (66.375 inch) wide, came from the same lot as the $0.759 \mathrm{~m}$ ( $29.875 \mathrm{inch}$ ) wide rolls used for the Beloit pilot liner.

Table $\mathrm{C} 4$ identifies the liner used in each of the corrugator cases. Corrugator case 1 was conducted with commericial linerboard. Corrugator cases 2A, 2B, 4, and 6 used impulsedried liner while cases 3 and 5 used the single-felted wet pressed control. The same medium was used for all corrugator cases. Note that case $2 \mathrm{~A}$ and $2 \mathrm{~B}$ were produced from different reels produced at the same nominal conditions. Table C5 shows some pertinent physical properties of the liner and medium used in each case.

The flexo, folder, gluer operation also proceeded quite smoothly after adjusting for proper ink coverage and printing pressure on all six sets of blanks. Finished containers were placed on pallets, shrink wrapped for final shipment, and tested at the Institute.

No problems were observed in the die cutting operation, and no score cracking problems noted in $M D, C D$, or angle scores. 


\section{C3.) Results}

Combined board and boxes produced for each case were numbered in the order of corrugating prior to the initiation of testing. Four sequential sets of samples were taken from each of the cases, resulting in a total of 28 sample sets.

\section{Lane and Sequence Effects}

Lane-specific testing of the liner demonstrated a CD profile in each of the reels produced on the Beloit pilot machine. The center lane typically was found to exhibit lower strength. It was therefore important to test the combined board in both the center and edge lanes for edge crush, caliper, and pin adhesion. The results of these tests are plotted versus corrugator sequence in Figures $\mathrm{C} 1$ through $\mathrm{C} 4$.

In Figure $\mathrm{C} 1$ the edge crush as measured in the center lane was typically lower than that measured on the edges for cases $2 A$ through 6 . This is consistent with the lower STFI compression strength and ring crush of the center lane of the liner. The edge crush of case 1, the commercial control, was position independent, as expected.

In Figure $\mathrm{C} 2$ the caliper of the combined board is reported for both the center and edges of the samples. There was a slight tendency for the center to be of lower caliper than the edges.

Single-facer pin adhesion data is reported in Figure C3. There was no observed bias regarding test position as the results in the center are similar to those on the edges. Double-backer pin adhesion data is reported in Figure C4. Here, the pin adhesion strength in the center was typically higher than at the edges. This was also true for the commercial control. Hence, it is probable that this bias was due to converting equipment rather than the liner.

Figure $\mathrm{C} 5$ shows the flexural stiffness of the combined board as measured in both the $M D$ and $C D$ directions. Due to the size of the test specimen, only the center was tested.

Figure $\mathrm{C} 6$ shows the peak load as measured during top-to-bottom box compression testing. Note that case 6 could not be included as we only made combined board blanks and did not make boxes in this case. Figure $\mathrm{C} 7$ shows the deflection at peak load as measured during the box compression testing.

\section{Case Effects}

In Figures C8 through C14, the four sequential sets in each case are pooled together to obtain average properties per case. Figure $C 8$ shows that the highest edge crush values were obtained for cases $1,2 A, 2 B$, and 4 , while the lowest edge crush values were obtained in cases 3,5 , and 6 .

Figure C 9 shows that the commercial liner yielded combined board with the highest caliper, while board made from the pilot produced reels were of consistently lower caliper. 
Figure $\mathrm{C} 10$ shows that the highest single-facer pin adhesion values were obtained for cases 3 and 5 where the single-felted wet pressed liner was utilized. Pin adhesion for the impulse dried cases were lower but consistent with that obtained using the commercial liner, see case 1. Figure $\mathrm{C} 11$ shows that the double-backer pin adhesion for board made from the pilot-produced liner was at least as strong as that obtained from the commercial sample, case 1.

Figure $\mathrm{C} 12$ shows that the flexural stiffness was increased when the liner was impulse dried. Further analysis (see below) shows that this was related to increases in Young's modulus of the impulse dried liner.

Figure $\mathrm{C} 13$ shows an improvement in box compression strength for the impulse dried cases $2 \mathrm{~A}, 2 \mathrm{~B}$ and 4 as compared to the wet pressed control cases 3 and 5 . The impulse dried cases are a least as strong as the commercial control. Boxes made from liner impulse dried at the highest temperature were superior in strength to those made from the wet pressed control as well as the commercial control.

Figure $\mathrm{C} 14$ shows that box defiection at peak load was fairly independent of case.

Table C6 shows the average edge crush, combined board caliper, and single-facer and double-backer pin adhesions for each case. Table $\mathrm{C} 7$ shows the percentage change for each of these as compared to the corresponding wet-pressed control. It is observed that impulse drying resulted in as much as a $10.6 \%$ increase in edge crush, a decrease in single-facer pin adhesion of as much as $27.3 \%$, and an increase in double-backer pin adhesion of as much as $9.5 \%$.

Table C8 shows the average peak load from top-to-bottom, end-to-end, and side-to-side box compression testing, as well as the MD and CD flexural stiffness of the combined board. Table 2-9 reports the percent change of these properties as compared to the appropriate wet pressed control. Impulse drying resulted in as much as a $10.3 \%$ increase in top-to-bottom box compression strength.

Visual inspection of the printed boxes showed significant improvement to print coverage. Impulse dried samples had superior print quality to boxes made from the wet pressed control liners as well as those made from the commercial liner.

\section{C4.) Discussion}

\section{Box Compression Strength}

In 1963 McKee [1] published an equation which could be used to predict box compression strength as a function of edge crush strength, flexural stiffness, and box perimeter.

The McKee equation is,

$P=2.028\left(P_{m}\right)^{0.746}\left(D_{x} D_{y}\right)^{0.127}(Z)^{0.492}$

Where,

$\mathrm{P} \quad=$ Box Compression Strength, $\mathrm{lb}$

$P_{m} \quad=$ Edge Crush Test Strength, $\mathrm{lb} / \mathrm{in}$

$D_{x} \quad=$ MD Flexural Stiffness, lb-in

$D_{y} \quad=$ CD Flexural Stiffness, lb-in 
$Z \quad=$ Box Perimeter, in

Based on the powers in the McKee formula, it is recognized that edge crush strength plays a dominant role in determining box compression strength. Flexural stiffness and box perimeter play lesser roles.

\section{Edge Crush}

For two grade ranges, Witsitt [2] has suggested equations for predicting edge crush from the ring crush of liner and medium used to manufacture corrugated board. These are given as,

ECT Grades 23-32 lb/in:

$P_{m}=0.80(2 L+t M)+12$

ECT Grades 38-60 lb/in:

$P_{m}=1.27(2 L+t M)-6$

Where,

$L \quad=C D$ ring crush of the linerboard, $\mathrm{lb} / \mathrm{in}$

$M=C D$ ring crush of the medium, $\mathrm{lb} / \mathrm{in}$

$\mathrm{t}=$ draw or take-up factor

A-flute $=1.55$

B-flute $=1.36$

C-flute $=1.42$

Whittsit also suggests an equation to predict edge crush from STFI compression strength,

$P_{m}=0.545\left(2 L_{s}+t M_{s}\right)+4.785$

Where,

$L_{s} \quad=$ CD STFI compression strength of the linerboard, lb/in

$\mathrm{M}_{\mathrm{s}} \quad=\mathrm{CD}$ STFI compression strength of the medium, $\mathrm{Ib} / \mathrm{in}$

Hence, assuming that corrugating conditions are normal, we can predict edge crush from linerboard and medium strength properties. If corrugating process variables deviate from normal practice, edge crush may also depend on these corrugating variables. Along these lines, Batelka [3] has developed a correlation for edge crush in terms of some box plant process variables. For the range of his experiments, the correlation was,

$P_{m}=33.7-a(A)+b(B)-c(C)-d(D)-e(E)$

Where,
$a=$
$0.0397, \quad A=$
Leaning Flute Angle, degrees
$b=$
$0.1500, \quad B=\quad$ Single-Face Pin Adhesion, lb
$c=$
$0.0534, \quad C=$
$d=$
$0.1340, \quad D=$
High/Low Flutes @ 4 mils or Greater, \%
$\mathrm{e}=$
$0.1300, \quad E=$ Actual Crushing, mils
Pressure Roll Cutting, \% Mullen Loss 
from the coefficients we see that single-face pin adhesion, flute crushing, and pressure roll cutting are the dominant factors.

\section{Flexural Stiffness}

Based on Whittsit's work [2] we can get additional insight into edge crush by exploring how flexural stiffness is related to the properties of the linerboard and medium as well as the geometry of the combined board. Whittsit gives the following equations as approximations,

$$
\begin{aligned}
& D_{x}=E_{x f} T H^{2} / 2 \\
& D_{y}=E_{y f} T H^{2} / 2+E_{y m} I
\end{aligned}
$$

Where,

$$
\begin{array}{ll}
\text { Dx, Dy } & =\text { Flexural stiffness in the MD and CD directions, Ib-in } \\
E_{x f}, E_{y f} & =\text { Young's modulus of the linerboard in the MD and CD directions, } \mathrm{Ib} / \mathrm{in}^{2} \\
T & =\text { Average linerboard thickness, in } \\
H & =\text { Combined board thickness, in } \\
E_{\mathrm{zm}} & =\text { Young's modulus of the medium in the CD direction, } \mathrm{lb} / \mathrm{in}^{2} \\
\mathrm{I} & =\text { Moment of inertia of the flute, } \mathrm{in}^{4} / \mathrm{in}
\end{array}
$$

In these equations, MD flexural stiffness is primarily dependent on the Young's modulus of the linerboard and the combined board caliper. In the CD direction, the flexural stiffness is dependent on the Young's modulus of the linerboard and the medium as well as the moment of inertia of the flute.

\section{Predicting Box Compression From Linerboard Properties}

Assuming normal corrugating conditions, box compression strength, $\mathrm{P}$, may be expressed as,

$P=2.028\left(P_{m}\right)^{0.746}\left(D_{x} D_{y}\right)^{0.127}(Z)^{0.492}$

In our experiments we have produced linerboard that has different properties than conventional linerboard. We expect the following properties of the linerboard to have changed; $L, E_{x f}, E_{y f}$, and $T$. Since the linerboard thickness changes, so will $H$, the combined board caliper. Using Equations $\{1\},\{2\},\{6\}$ and $\{7\}$, the change in $P$ may be calculated from measured changes in $L, E_{x f}, E_{y f}, T$, and $H$. By differentiation of $P$, we obtain,

$\Delta \mathrm{P}=(\delta \mathrm{P} / \delta \mathrm{L}) \Delta \mathrm{L}+(\delta \mathrm{P} / \delta \mathrm{Exf}) \Delta \mathrm{E}_{\mathrm{xf}}+\left(\delta \mathrm{P} / \delta \mathrm{E}_{\mathrm{yf}}\right) \Delta \mathrm{Eyf}+(\delta \mathrm{P} / \delta \mathrm{T}) \Delta \mathrm{T}+(\delta \mathrm{P} / \delta \mathrm{H}) \Delta \mathrm{H}$ 
Taking the partial derivatives,

$$
\begin{aligned}
& (\delta P / \delta L)=1.1936\left\{P / P_{m}\right\}=\{1.1936 /(0.80(2 L+t M)+12)\} P \\
& \left(\delta P / \delta E_{x f}=0.0635\left\{T H^{2} / D_{x}\right\} P=\left\{0.127 / E_{x f}\right\} P\right. \\
& \left(\delta P / \delta E_{y f}\right)=0.0635\left\{T H^{2} / D_{y}\right\} P=0.0635\left\{T H^{2} /\left(E_{y f}\left(T H^{2} / 2\right)+E_{y m}\right)\right\} P \\
& (\delta P / \delta T)=\{0.254 / T\}\left\{\left(E_{y y} H^{2} T+E_{y m} l\right) /\left(E_{y} H^{2} T+2 E_{y m} l\right)\right\} P \\
& (\delta P / \delta H)=0.508\left\{\left(E_{y y} H^{2} T+E_{y m} \mid\right) /\left(E_{y f} H^{3} T+2 E_{y m} H I\right)\right\} P
\end{aligned}
$$

Based on the linerboard data and the above equations, Table C10 was constructed.

Here we have assumed that the following properties were constant;

$$
\begin{aligned}
& \mathrm{M} \quad=5.10 \mathrm{lb} / \mathrm{in} \\
& \mathrm{E}_{\mathrm{ym}} \quad=10222 \mathrm{lb} / \mathrm{in}^{2} \\
& \mathrm{t}=1.42 \\
& 1=0.0002863 \mathrm{in}^{4} / \mathrm{in} \text {, (estimated, see [4]) }
\end{aligned}
$$

From Table $\mathrm{C} 10$, the theory predicts that use of the impulse dried linerboard would yield a $17.14 \mathrm{lb}$ increase in box compression strength compared to the case where the wet pressed linerboard was used. Most of the increase comes from the increase in ring crush with smaller increases from the Young's modulus terms. It is noted that the reduced linerboard thickness yields a negative contribution to box compression strength that is less than half of the magnitude of the ring crush term. It is also noted that, in actuality, we measured an increase in box compression strength of $42.72 \mathrm{lb}$. The discrepancy with the theory may result from the fact that the equations used are based on correlations. In any case, impulse drying yielded higher box compression strength because of the increase in ring crush (or STFI) and to a lesser extent from increases in Young's modulus of the linerboard.

\section{Pin Adhesion Strength}

In Equation $\{5\}$ we saw the importance of pin adhesion as a corrugation variable. Hence, we present a brief summary of some key points in the literature on pin adhesion.

According to Daub [5], glueability is the ability of paper to anchor and to permit setting of the glue, which is determined by the setting mechanism. For starch glue, the setting mechanism is gelatinization, which requires heat and water. The main parameters of the gluing process are the amount of glue applied (or spread), the joining pressure, heat, and the open time and closed time as determined by web speed. He reports that the paper properties which probably affect the glueability are basis weight, apparent density, moisture content, porosity, absorbency, thermal conductivity, surface energy, and roughness. The absorbency behavior of paper is different for glue and water. Looking at it another way, it also depends on the capillary forces and external pressure.

Highton [6] has presented a theoretical analysis of the physical conditions to which fluted medium is subjected as it passes through a single-facer. He states that the critical part of the single-facer process is the impact of the pressure roll forcing the liner against the glued flute tip of the fluting medium. The openness, density, and compressibility of the 
linerboard and medium determines how far the glue is forced into the fluting medium and liner and how much of the glue is squeezed out onto the shoulders of the flute. The density and compressibility of the liner and medium also control how much heat from the corrugator roll and pressure roll is conducted into the glue zone of the joint.

Lepoutre [7] has reviewed the principles of adhesion and has examined the main aspects of glueability at the corrugator. He reports that penetration at the single-facer is mainly a function of pressure. While pressure plays a much less important role in penetration at the double-backer.

Rutherford [8] has reported on the effect that the use of high-performance (high-density) liners has had on pin adhesion. He states that the downside of high-performance liners is that densification leads to fewer and smaller openings in the surface of the sheet. Thus, it becomes difficult for the corrugating adhesive to work its way into the surface. Such paper will be more difficult to bond, producing shallow surface bonds, at best, if corrugating conditions are not altered.

Wallace [9] has found that high-performance liners, with their increased density and reduced moisture content, offer improved ring crush. However, because of their increased density and reduced moisture content, they are more prone to overheating, poor adhesive interaction, and dry cracking. These high-performance liners are produced on paper machines with extended nip presses. With these presses, and as press loading is increased, the sheet becomes more compressed, and interfiber bonding (hydrogen bonding) improves. The sheet becomes tighter and denser, resulting in higher ring crush values. However, the increased density also affects other properties of the paper. Highdensity liners have closer fibers, making the sheet much less porous than conventional kraft papers. Reducing porosity in the sheet increases the potential of bonding problems because of poor adhesive penetration. In addition, the moisture content of the highperformance liners is typically lower than conventional kraft liners ( 4 to $4.5 \%$ compared to 8 to $9 \%$ ). In addition, with closer fiber contact and less insulating air space, heat easily passes through high-density paper. Thus these liners become hotter more quickly than conventional liners. Wallace reports that these problems can be overcome by adding moisture to the liner and medium and by careful control of temperature.

In the box plant trial, the single-facer pressure was set by fixing the clearance between the pressure roll and the upper corrugator roll. Hence, the actual applied pressure increased with increased caliper of the liner, and single-facer pin adhesion decreased with decreased caliper or increased apparent density as we have observed (see Figure 2-15). Hence, we expect that we could have increased single-facer pin adhesion of the impulse dried cases by suitable adjustment of pressure.

\section{C5.) References}

1. McKee, R.C., Gander, J.W., and Wachuta, J. R., "Compression Strength Formula for Corrugated Boxes," Paperboard Packaging 48(8):149-159 (August 1963).

2. Whitsitt, W. J., "Corrugated Board and Box Performance," Chapter 20, Paper Properties and Their Measurement, IPST, (October 1990).

3. Batelka, J. J., "Corrugating Medium: Its Influence on Box Plant Operations and Package Performance," Project 3808, Report to the Containerboard and Kraft Paper Group of the American Forest and Paper Association from the Institute of Paper Science and Technology, 1993. 
4. Koning, J.W. and Moody R.C., "Predicting Flexural Stiffness of Corrugated Fiberboard," Tappi J. 54(11):1879-1881(November 1971).

5. Daub, E., Hoke, U., and Gottsching, L., "Gluing Corrugating Medium and Linerboard Together on the Corrugator," Tappi J. 73(6): 171-178 (June 1990).

6. Highton, P. A., "Physical Aspects of Single-Facer Operation," Tappi J. 76(7): 130-139 (July 1993).

7. Lepoutre, P. and Inoue, M., "Glueability at the Corrugator," Tappi J. 72(11): 113-120 (November 1989).

8. Rutherford, J. H., "The Evolution to High Performance Liners," MARI Board Corrugating News, 5(6): 47 (1992).

9. Wallace, J. R., Brombeerek, A. E., and Young, S. N., "High Density Papers / Corrugator Runnability," Proceedings of the 1993 International Corrugated Containers Conference, pp. 15-23, (1993).

\section{C6.) Acknowledgements}

The author would like to thank the Member Companies of the Institute of Paper Science and Technology, the Beloit Corporation, and the U.S. Department of Energy (through Grant No. DOE/CE/40738) for supporting this research. The author would also like to thank Chuck Boswell, Bill Sampson, and David Carlson of Stone Container, and Joseph Schlinkert and Bill Morrissey of Borden Chemical for their contributions to this work.

\section{C7.) Tables}

Table C1. Corrugating Conditions

\begin{tabular}{|c|c|}
\hline Manufacturer & Langston \\
\hline Type & XD \\
\hline Width & $2.21 \mathrm{~m}(87 \mathrm{inch})$ \\
\hline Run Speed & $121.9 \mathrm{~m} / \mathrm{min}(400 \mathrm{ft} / \mathrm{min})$ \\
\hline Liner Width & $0.762 \mathrm{~m}(30 \mathrm{inch})$ \\
\hline Liner Basis Weight & $161 \mathrm{~g} / \mathrm{m}^{2}(33 \mathrm{lb} / \mathrm{msf})$ \\
\hline Medium Width & $0.759 \mathrm{~m}(29.875 \mathrm{inches})$ \\
\hline Medium Type & $40 \%$ nonsulfur $-60 \%$ OCC \\
\hline Medium Basis Weight & $126 \mathrm{~g} / \mathrm{m}^{2}(26 \mathrm{lb} / \mathrm{msf})$ \\
\hline Starch for Single-facer & modified pearl, $26 \%$ solids, \\
& $62.2^{\circ} \mathrm{C}\left(144^{\circ} \mathrm{F}\right) \mathrm{Gel}$ Point \\
\hline Starch for Double-backer & $20 \%$ cooked, $80^{\circ}$ raw, $26 \%$ solids, \\
& $64.4^{\circ} \mathrm{C}\left(148^{\circ} \mathrm{F}\right) \mathrm{Gel} \mathrm{point}$ \\
\hline Anilox roll & 1.378 lines $/ \mathrm{mm}(35$ lines $/$ inch $)$ \\
\hline
\end{tabular}


Table C2. Printing Conditions

\begin{tabular}{|c|c|}
\hline Manufacturer & Ward Machinery \\
\hline Width & $1.27 \mathrm{~m}(50$ inch $) \times 2.79 \mathrm{~m}(110 \mathrm{inch})$ \\
\hline Blank size & $1.52 \mathrm{~m}(60$ inch $) \times 0.69 \mathrm{~m}(27$ inch $)$ \\
\hline Speed & 80 kicks per minute \\
\hline Ink & GCMI black (Borden Chemical Co. $)$ \\
\hline Anilox & 6.299 lines/mm $(160$ lines/inch) \\
\hline
\end{tabular}

Table C3. Die Cutting Conditions

\begin{tabular}{|c|c|}
\hline Manufacturer & United Container Machinery Group \\
\hline Size & $1.27 \mathrm{~m}$ (50 inch) \\
\hline
\end{tabular}

Table C4. Identification of Liner and Medium Used

\begin{tabular}{|c|c|c|c|c|}
\hline Paper Type & $\begin{array}{c}\text { Third Press } \\
\text { Type }\end{array}$ & $\begin{array}{c}\text { Impulse Drying } \\
\text { Temperature, } \\
{ }^{\circ} \mathrm{C}\end{array}$ & Calendering & $\begin{array}{c}\text { Used In Case } \\
\text { Number }\end{array}$ \\
\hline Liner & Commercial & & & 1 \\
\hline Liner & Impulse-Dried & 246 & no & $2 \mathrm{~A}$ \\
\hline Liner & Impulse-Dried & 246 & no & $2 \mathrm{~B}$ \\
\hline Liner & S.F. Wet Press & & yes & 3 \\
\hline Liner & Impulse-Dried & 260 & no & 4 \\
\hline Liner & S.F. WetPress & & no & 5 \\
\hline Liner & Impulse-Dried & 246 & yes & 6 \\
\hline Medium & Commercial & & & $1-6$ \\
\hline
\end{tabular}

Table C5. Physical Properies Of The Liner And Medium

\begin{tabular}{|c|c|c|c|c|c|c|}
\hline Case & $\begin{array}{c}\text { O.D. } \\
\text { Basis } \\
\text { Weight, } \\
\mathrm{g} / \mathrm{m}^{2}\end{array}$ & $\begin{array}{c}\text { Soft } \\
\text { Caliper }\end{array}$ & $\begin{array}{c}\mathrm{CD} \\
\text { STFI } \\
\text { Index, } \\
\mathrm{Nm} / \mathrm{g}\end{array}$ & $\begin{array}{c}\mathrm{C} \\
\text { Ring } \\
\text { Crush } \\
\text { Index, } \\
\mathrm{Nm} / \mathrm{g}\end{array}$ & Burst, & $\begin{array}{c}\text { Printed Side } \\
\text { Emveco } \\
\text { Roughness, } \\
\text { Micro } \\
\text { Deviation }\end{array}$ \\
\hline Liner-1 & 147 & 210 & 21.6 & 12.0 & 574 & 184 \\
\hline Liner-2A & 150 & 218 & 19.4 & 11.9 & 539 & 85 \\
\hline Liner-2B & 150 & 218 & 19.4 & 11.9 & 539 & 85 \\
\hline Liner-3 & 151 & 238 & 17.6 & 10.4 & 445 & 123 \\
\hline Liner -4 & 150 & 218 & 19.4 & 11.9 & 521 & 81 \\
\hline Liner -5 & 152 & 246 & 17.6 & 10.7 & 460 & 184 \\
\hline Liner -6 & 150 & 208 & 19.2 & 11.8 & 533 & 69 \\
\hline Medium - (1-6) & 115 & 185 & 16.6 & 7.7 & 243 & n.m. \\
\hline
\end{tabular}


Table C6. Combined Board Properties

\begin{tabular}{|c|c|c|c|c|}
\hline $\begin{array}{c}\text { Case } \\
\text { No. }\end{array}$ & $\begin{array}{c}\text { Average } \\
\text { Single- } \\
\text { Face Pin } \\
\text { Adhesion, } \\
\mathrm{Nm}\end{array}$ & $\begin{array}{c}\text { Average } \\
\text { Double- } \\
\text { Back Pin } \\
\text { Adhesion, } \\
\mathrm{Nm}\end{array}$ & $\begin{array}{c}\text { Average } \\
\text { Combined } \\
\text { Caliper, } \\
\mathrm{mm}\end{array}$ & $\begin{array}{c}\text { Average } \\
\text { Edge } \\
\text { Crush, } \\
\text { kN/m }\end{array}$ \\
\hline 1 & 589 & 606 & 3.995 & 5.230 \\
\hline $2 \mathrm{~A}$ & 534 & 693 & 3.810 & 5.095 \\
\hline $2 \mathrm{~B}$ & 557 & 740 & 3.680 & 5.125 \\
\hline 3 & 660 & 729 & 3.725 & 4.640 \\
\hline 4 & 565 & 642 & 3.710 & 5.075 \\
\hline 5 & 735 & 677 & 3.765 & 4.635 \\
\hline 6 & 599 & 699 & 3.605 & 4.685 \\
\hline
\end{tabular}

Table C7. Percent Change Compared To Wet Pressed Control

\begin{tabular}{|c|c|c|c|}
\hline $\begin{array}{c}\text { Case } \\
\text { No. }\end{array}$ & $\begin{array}{c}\text { Average } \\
\text { Single- } \\
\text { Facer Pin } \\
\text { Adhesion, } \\
\text { \& Change }\end{array}$ & $\begin{array}{c}\text { Average } \\
\text { Double- } \\
\text { Facer Pin } \\
\text { Adhesion, } \\
\% \text { Change }\end{array}$ & $\begin{array}{c}\text { Average } \\
\text { Edge } \\
\text { Crush, } \\
\% \text { Change }\end{array}$ \\
\hline 1 & & & \\
\hline $2 \mathrm{~A}$ & -27.3 & 2.4 & 9.9 \\
\hline $2 \mathrm{~B}$ & -24.2 & 9.3 & 10.6 \\
\hline 3 & & & \\
\hline 4 & -23.1 & -5.2 & 9.5 \\
\hline 5 & & & \\
\hline 6 & -9.3 & -4.1 & 1.0 \\
\hline
\end{tabular}

Table C8. Combined Board and Box Properties

\begin{tabular}{|c|c|c|c|c|c|}
\hline $\begin{array}{c}\text { Case } \\
\text { No. }\end{array}$ & $\begin{array}{c}\mathrm{MD} \\
\text { Flexural } \\
\text { Stiffness, } \\
\mathrm{Nm}\end{array}$ & $\begin{array}{c}\text { CD } \\
\text { Flexural } \\
\text { Stiffness, } \\
\mathrm{Nm}\end{array}$ & $\begin{array}{c}\text { Top-to-Bot } \\
\text { Box Compr. } \\
\text { Peak Load, } \\
\text { WN }\end{array}$ & $\begin{array}{l}\text { End-to-End } \\
\text { Box Compr. } \\
\text { Peak Load, } \\
\text { WN }\end{array}$ & $\begin{array}{l}\text { Side-to-Side } \\
\text { Box Compr. } \\
\text { Peak Load, } \\
\text { WN }\end{array}$ \\
\hline 1 & 9.83 & 4.97 & 1.99 & 1.27 & 1.62 \\
\hline $2 \mathrm{~A}$ & 8.70 & 4.32 & 2.00 & 1.36 & 1.64 \\
\hline $2 \mathrm{~B}$ & 9.12 & 3.92 & 2.04 & 1.17 & 1.54 \\
\hline 3 & 8.90 & 3.57 & 1.85 & 1.26 & 1.44 \\
\hline 4 & 9.82 & 4.30 & 2.14 & 1.41 & 1.76 \\
\hline 5 & 8.82 & 3.80 & 1.94 & 1.33 & 1.67 \\
\hline 6 & 8.80 & 4.04 & Not avail. & Not avail & Not avail. \\
\hline
\end{tabular}


Table C9. Percent Change Compared To Wet Pressed Control

\begin{tabular}{|c|l|l|c|c|c|}
\hline Case & $\begin{array}{l}\text { MD } \\
\text { Flexural } \\
\text { Stiffness, } \\
\text { \% Change }\end{array}$ & $\begin{array}{l}\text { F } \\
\text { Flexural } \\
\text { Stiffness, } \\
\% \text { Change }\end{array}$ & $\begin{array}{l}\text { Top-to-Bot } \\
\text { Box Compr. } \\
\text { Peak Load, } \\
\% \text { Change }\end{array}$ & $\begin{array}{c}\text { End-to-End } \\
\text { Box Compr. } \\
\text { Peak Load, } \\
\text { \% Change }\end{array}$ & $\begin{array}{c}\text { Side-to-Side } \\
\text { Box Compr. } \\
\text { Peak Load, } \\
\% \text { Change }\end{array}$ \\
\hline 1 & & & & & \\
\hline $2 \mathrm{~A}$ & -1.36 & 13.68 & 3.09 & 2.25 & -1.79 \\
\hline $2 \mathrm{~B}$ & 3.40 & 3.16 & 5.15 & -12.03 & -7.78 \\
\hline 3 & & & & & \\
\hline 4 & 11.34 & 13.16 & 10.31 & 6.01 & 5.39 \\
\hline 5 & & & & & \\
\hline 6 & -1.12 & 13.17 & Not avail. & Not avail. & Not avail. \\
\hline
\end{tabular}

Table C10. Measured and Predicted Change In Properties

\begin{tabular}{|l|c|c|c|}
\hline Property & $\begin{array}{c}\text { Wet Press Control } \\
\text { (case 3) }\end{array}$ & $\begin{array}{c}\text { Impulse Dried } \\
\text { (case 2B) }\end{array}$ & $\begin{array}{c}\text { Measured Change } \\
\Delta(\text { Property) }\end{array}$ \\
\hline $\mathrm{L}, \mathrm{kN} / \mathrm{m}$ or $(\mathrm{b} / \mathrm{in})$ & $1.57(8.97)$ & $1.78(10.17)$ & $0.21(+1.20)$ \\
\hline Exf, $\mathrm{MN} / \mathrm{m}$ or $\left(\mathrm{lb} / \mathrm{in}^{2}\right)$ & $360.5(52292)$ & $375.7(54483)$ & $15.2(+2191)$ \\
\hline Eyf, $\mathrm{MN} / \mathrm{m}$ or $\left(\mathrm{b} / \mathrm{in}^{2}\right)$ & $132.3(19189)$ & $146.4(20957)$ & $14.1(+1768)$ \\
\hline $\mathrm{T}, \mathrm{mm}$ or $(\mathrm{in})$ & $0.24(0.0094)$ & $0.22(0.0086)$ & $-0.02(-0.0008)$ \\
\hline $\mathrm{H}, \mathrm{mm}$ or $(\mathrm{in})$ & $3.73(0.1470)$ & $3.68(0.1450)$ & $-0.05(-0.0020)$ \\
\hline $\mathrm{P}, \mathrm{kN}$ or $(\mathrm{lb})$ & $1.85(415.9)$ & $2.04(458.61)$ & $0.19(+42.71)$ \\
\hline$(\delta \mathrm{P} / \delta \mathrm{L}) \Delta \mathrm{L}, \mathrm{kN}$ or $(\mathrm{lb})$ & $0.082(+18.53)$ & & \\
\hline$\left(\delta \mathrm{P} / \delta \mathrm{E}_{\mathrm{xf}}\right) \Delta \mathrm{E}_{\mathrm{xf}, \mathrm{kN} \text { or }(\mathrm{bb})}$ & $0.010(+2.21)$ & & \\
\hline$\left(\delta \mathrm{P} / \delta \mathrm{E}_{\mathrm{yf}}\right) \Delta \mathrm{E}_{\mathrm{yf}} \mathrm{kN}$ or $(\mathrm{bb})$ & $0.009(+1.94)$ & & \\
\hline$(\delta \mathrm{P} / \delta \mathrm{T}) \Delta \mathrm{T}, \mathrm{kN}$ or $(\mathrm{lb})$ & $-0.028(-6.21)$ & & \\
\hline$(\delta \mathrm{P} / \mathrm{HH}) \Delta \mathrm{H}_{,} \mathrm{kN}$ or $(\mathrm{lb})$ & $0.003(+0.67)$ & & \\
\hline
\end{tabular}




\section{C8.) Figures}

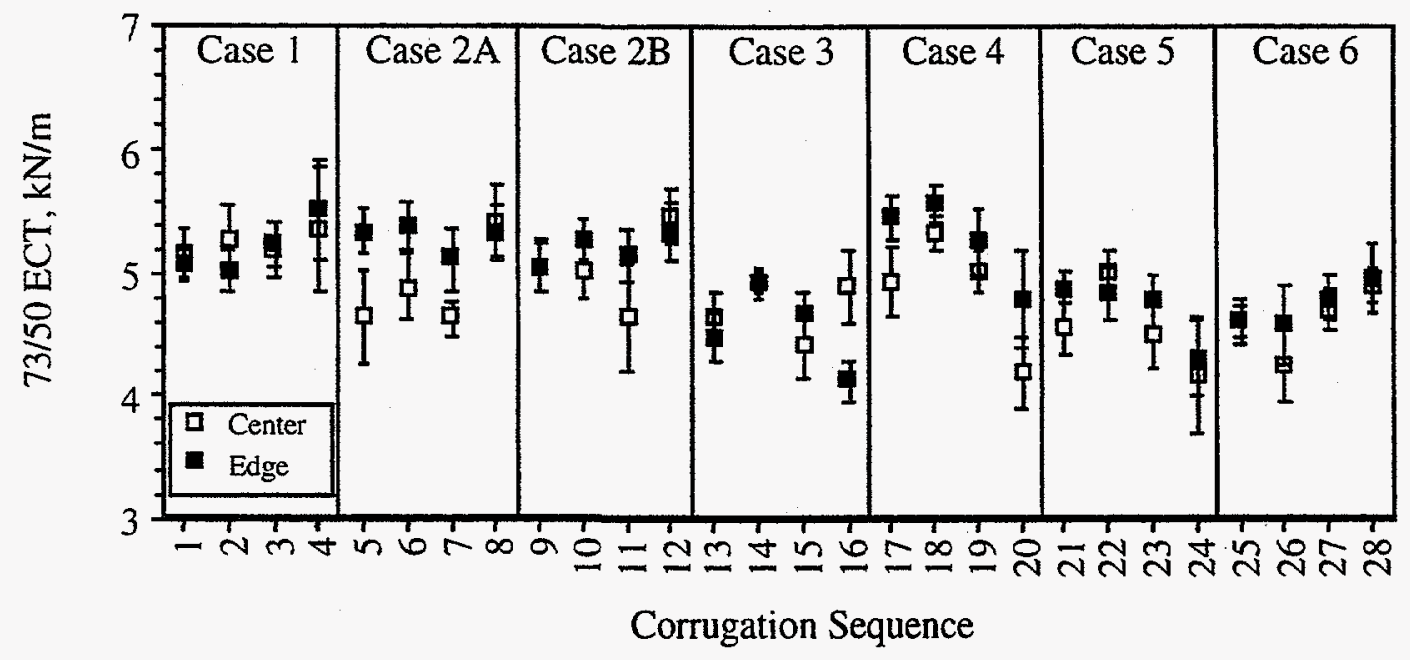

Figure C1. Edge Crush Test Of Corrugated Board (As Measured in The Center And On The Edges Of The Board) Plotted Versus Corrugation Sequence.

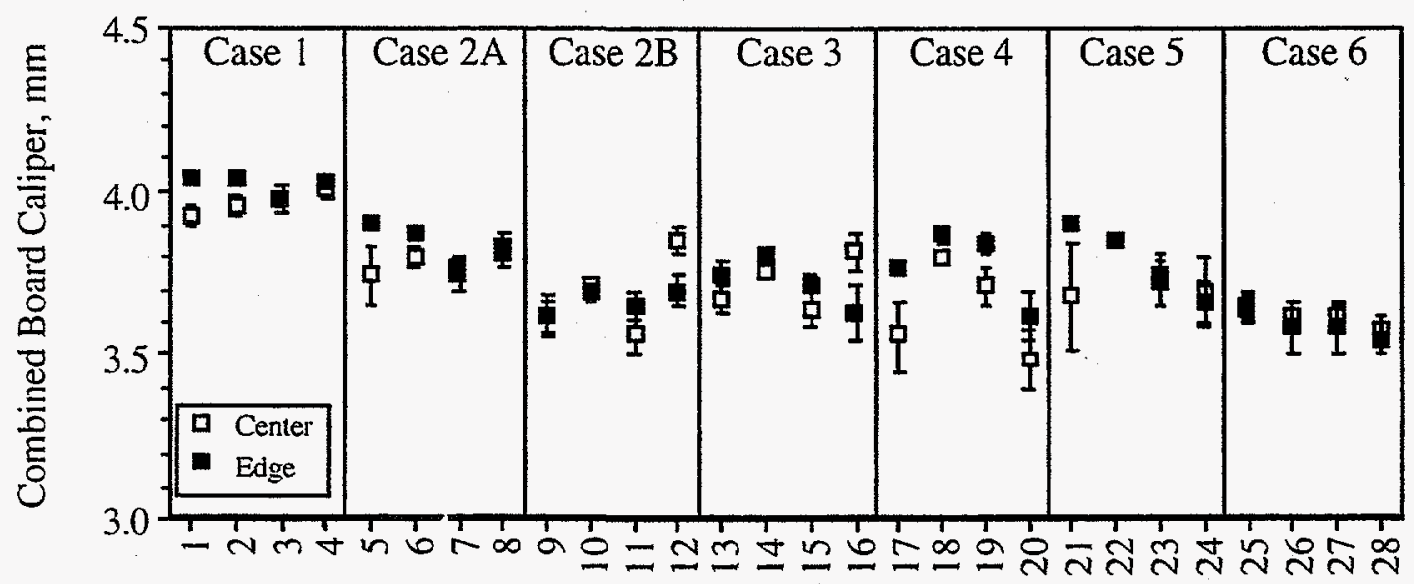

Corrugation Sequence

Figure C2. Caliper Of Corrugated Board (As Measured In The Center And On The Edges Of The Board) Plotted Versus Corrugation Sequence. 


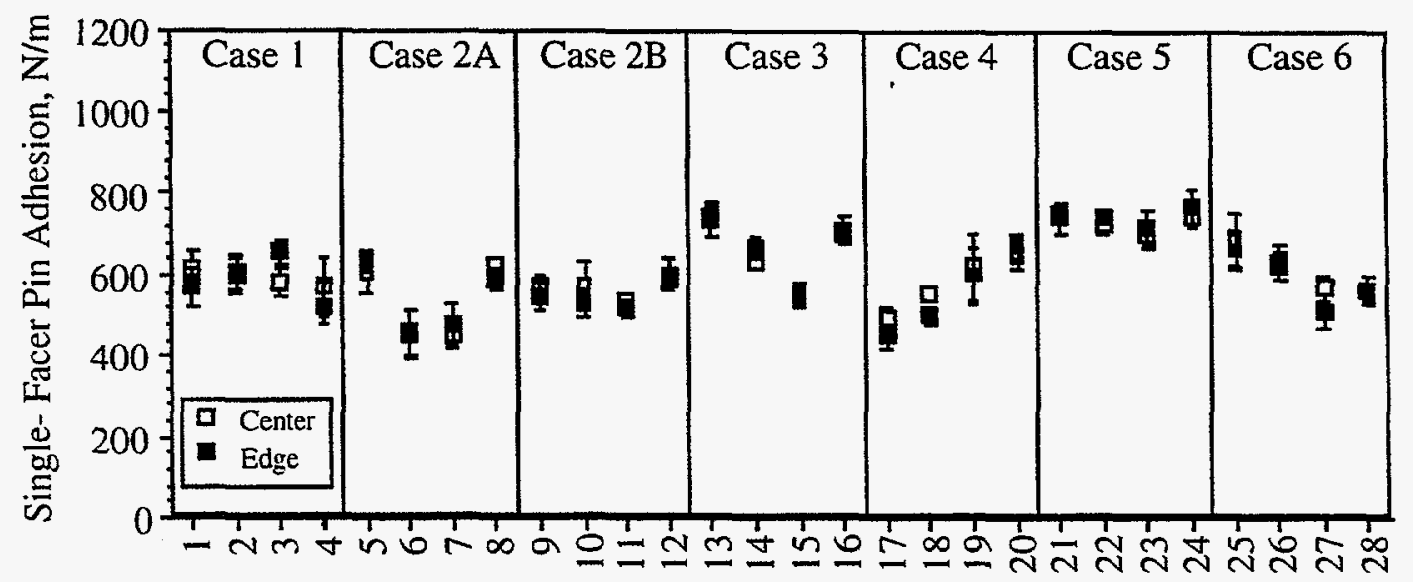

Corrugation Sequence

Figure C3. Single-Facer Pin Adhesion Of Corrugated Board (As Measured In The Center And On The Edges Of The Board) Plotted Versus Corrugation Sequence.

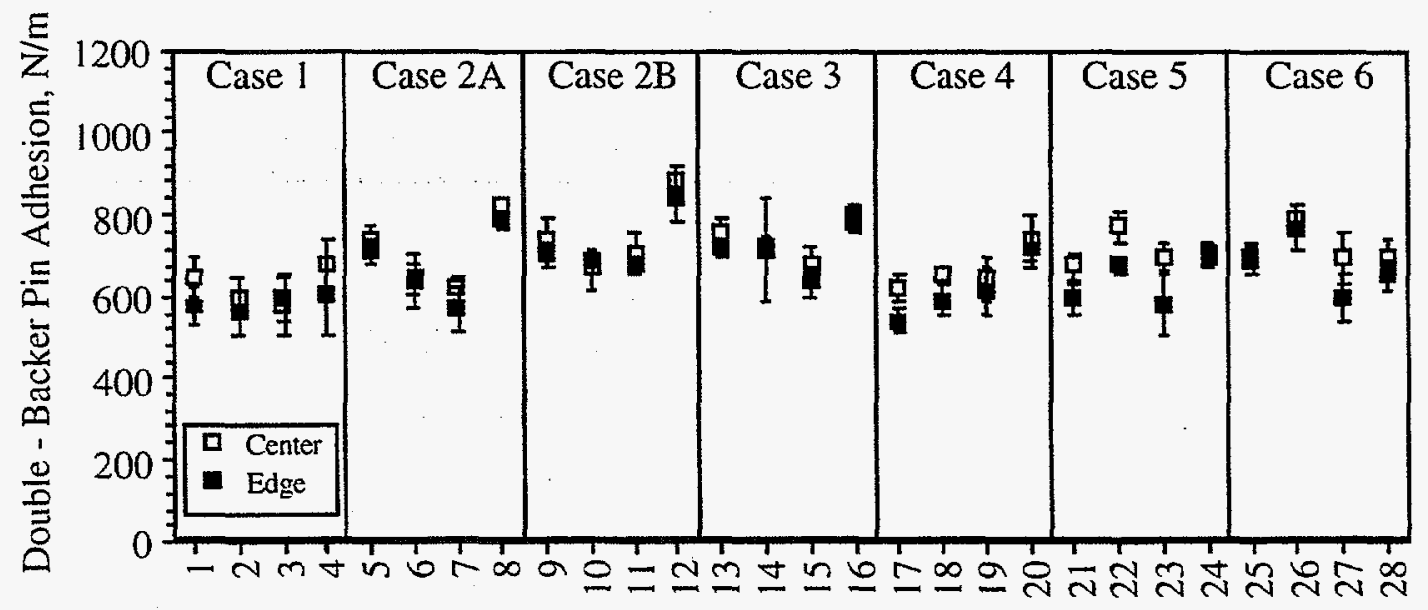

Corrugation Sequence

Figure C4. Double-Backer Pin Adhesion Of Corrugated Board (As Measured In The Center And On The Edges Of The Board) Plotted Versus Corrugation Sequence. 


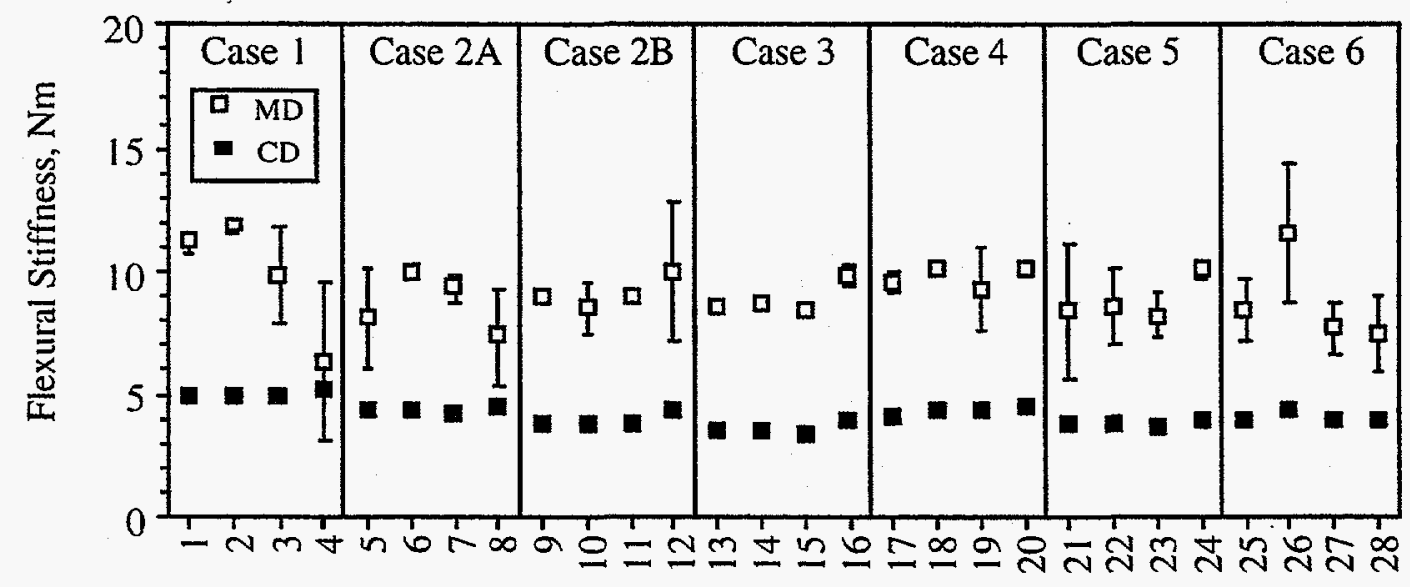

Corrugation Sequence

Figure C5. Flexural Stiffness Of Corrugated Board (As Measured In MD and CD Directions) Plotted Versus Corrugation Sequence.

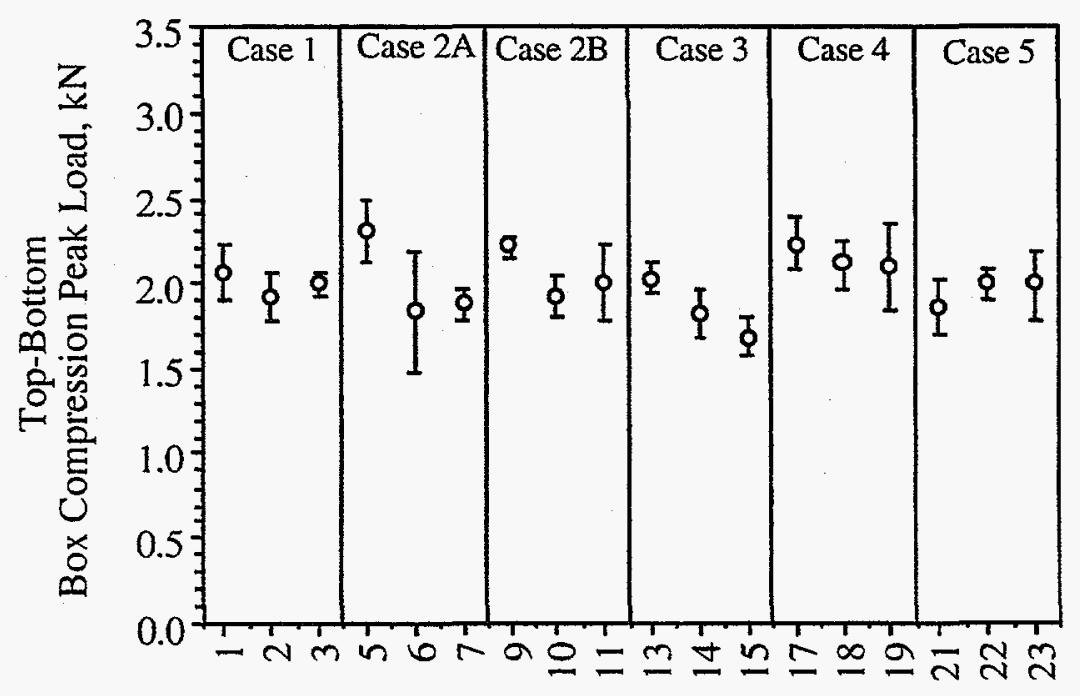

Corrugation Sequence

Figure C6. Top-To-Bottom Box Compression Peak Load Plotted Versus Corrugation Sequence. 


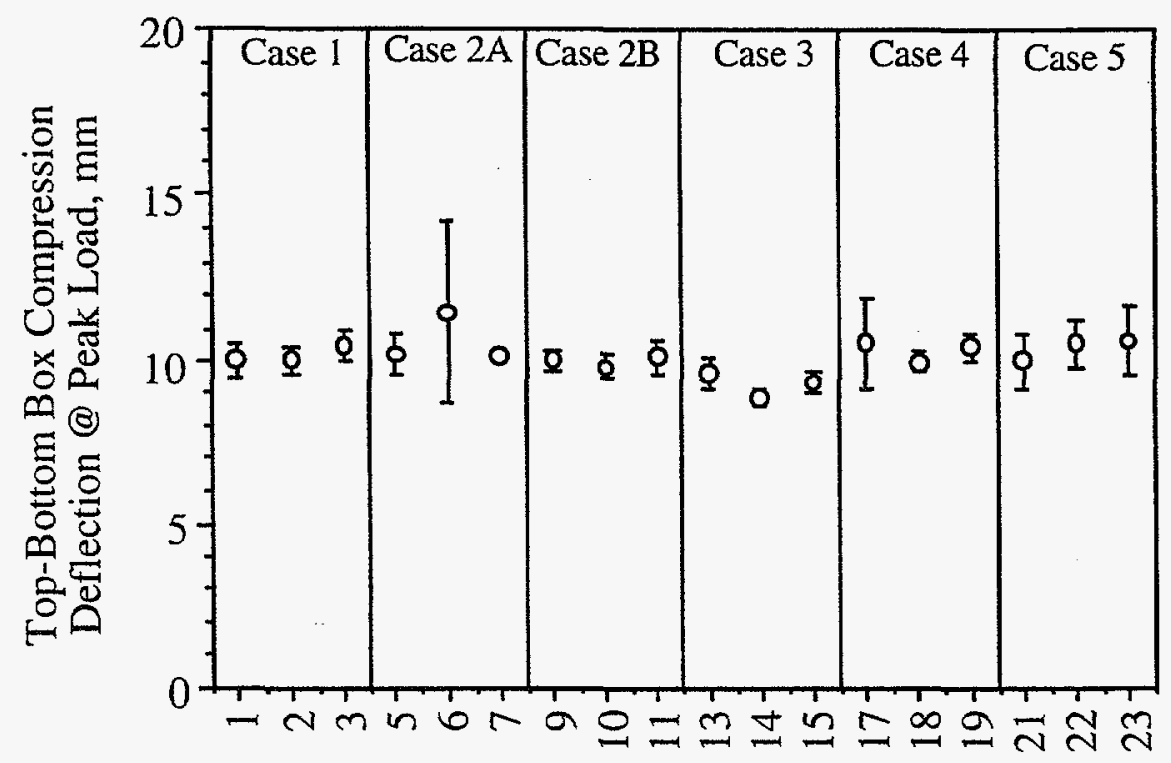

Corrugation Sequence

Figure C7. Top-To-Bottom Box Compression Deflection at Peak Load Plotted Versus Corrugation Sequence.

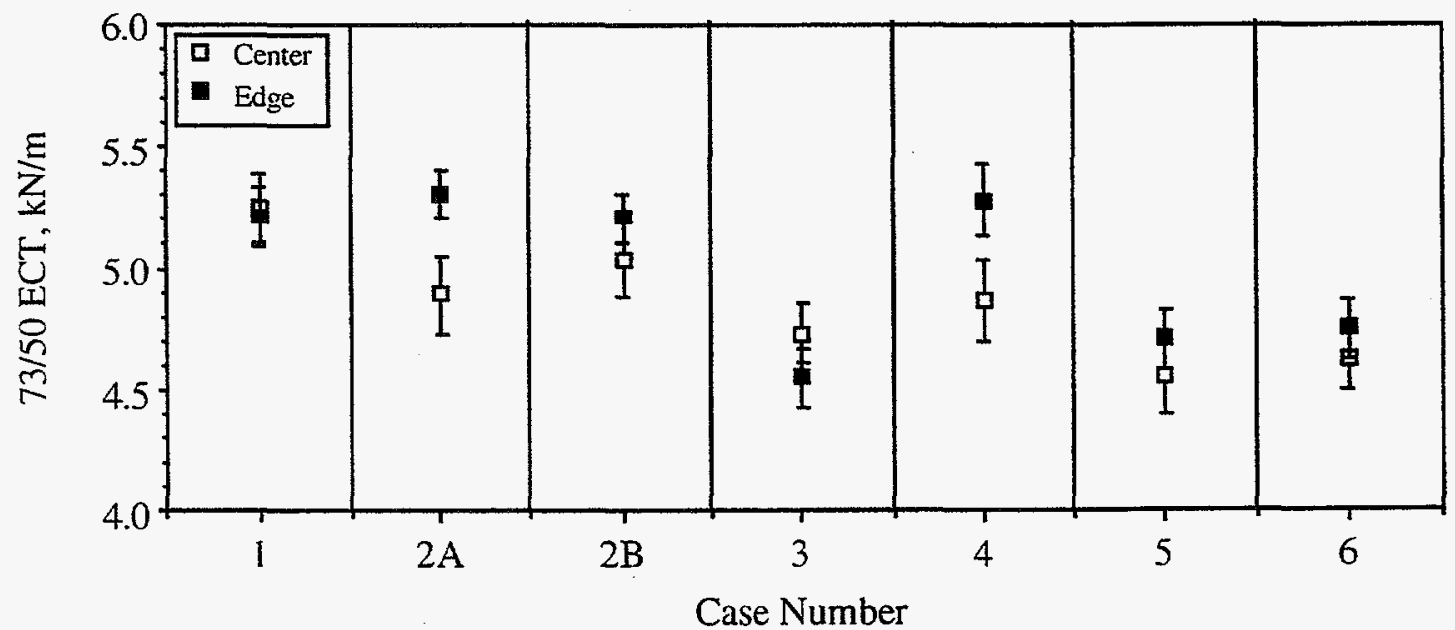

Figure C8. Edge Crush Test Of Corrugated Board (As Measured In The Center And On The Edges Of The Board) Plotted Versus Corrugation Case Number. 


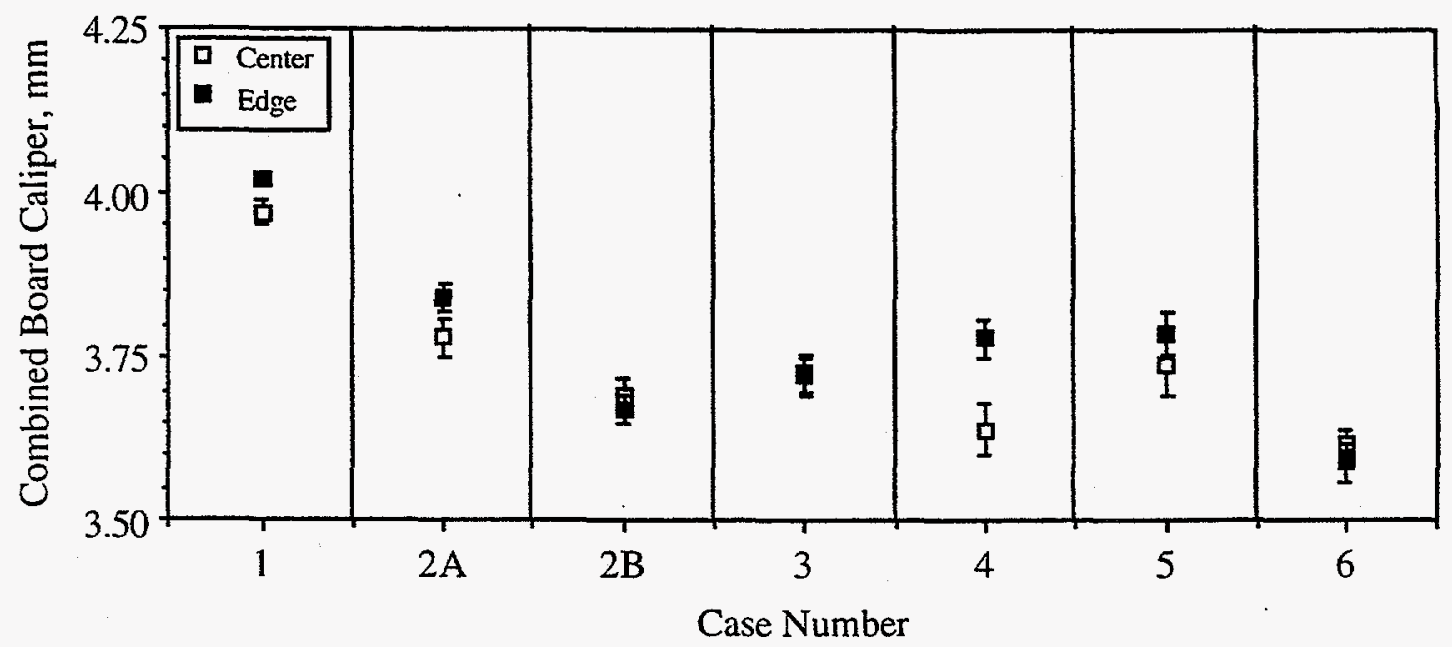

Figure C9. Caliper Of Corrugated Board (As Measured In The Center And On The Edges Of The Board) Plotted Versus Corrugation Case Number.

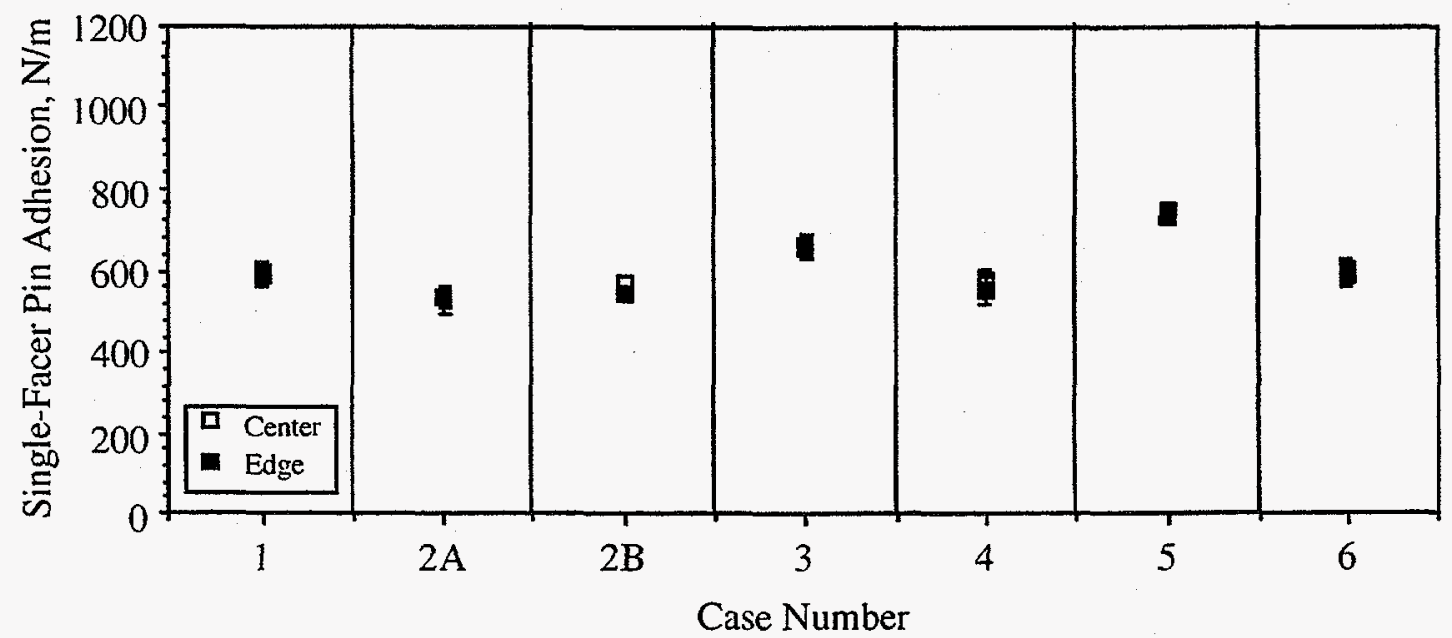

Figure C10. Single-Facer Pin Adhesion Of Corrugated Board (As Measured In The Center And On The Edges Of The Board) Plotted Versus Corrugation Case Number. 


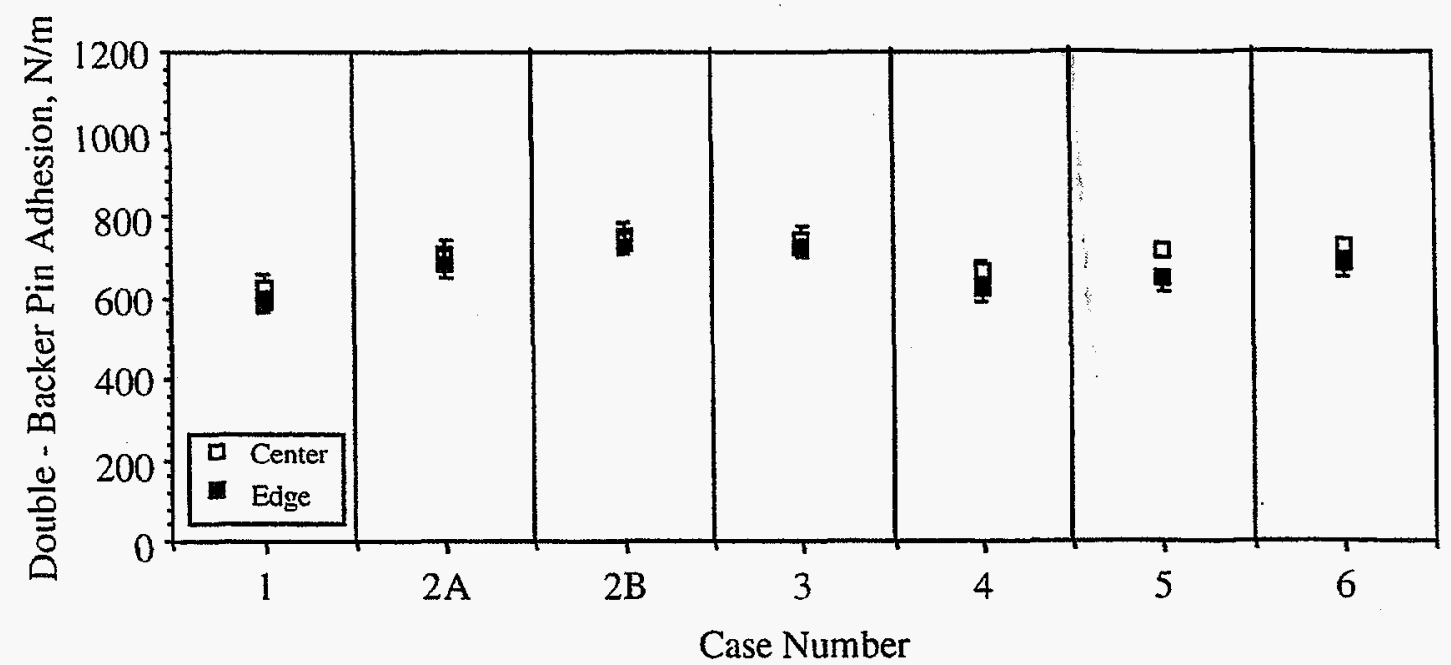

Figure C11. Double-Backer Pin Adhesion Of Corrugated Board (As Measured In The Center And On The Edges Of The Board) Plotted Versus Corrugation Case Number.

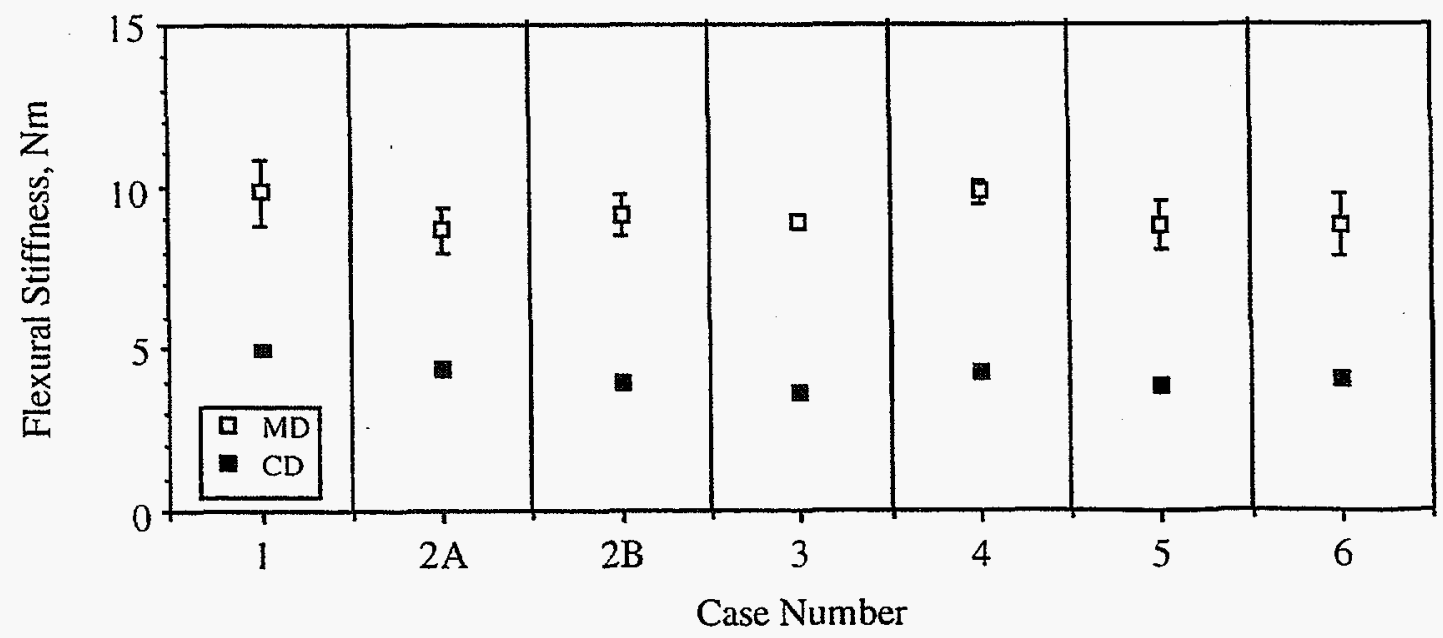

Figure C12. Flexural Stiffness Of Coriugated Board (As Measured In MD and CD Directions) Plotted Versus Corrugation Case Number. 


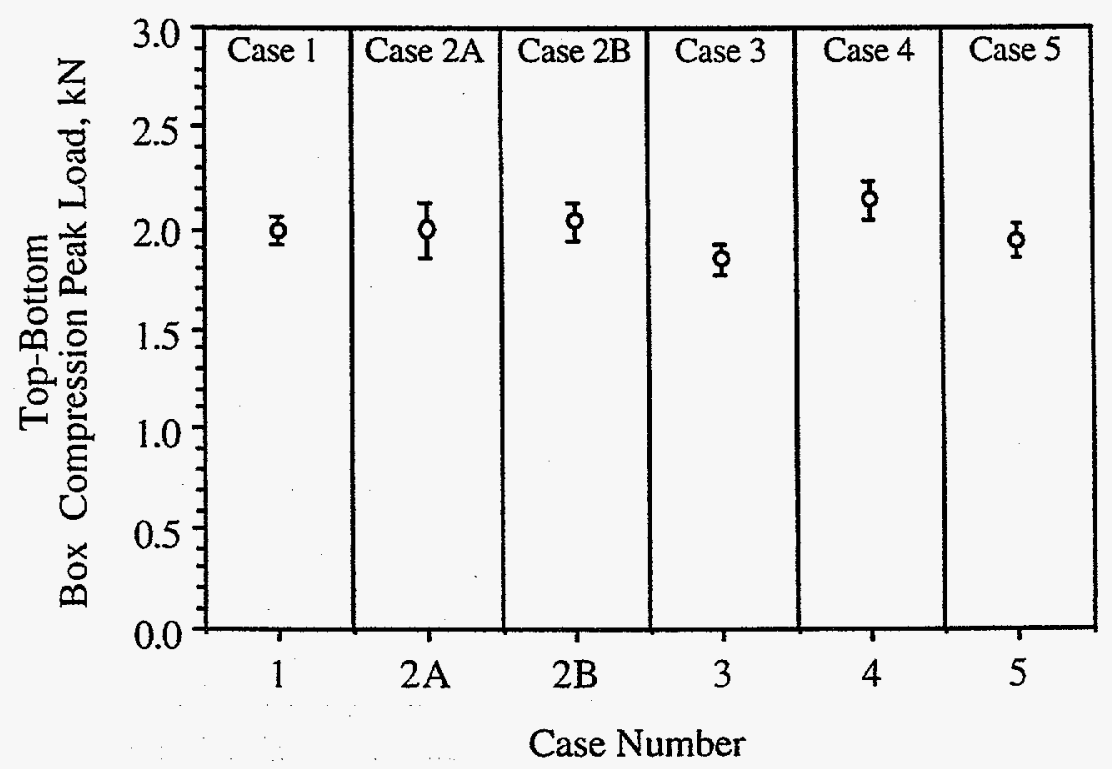

Figure C13. Top-To-Bottom Box Compression Peak Load Plotted Versus Corrugation Case Number.

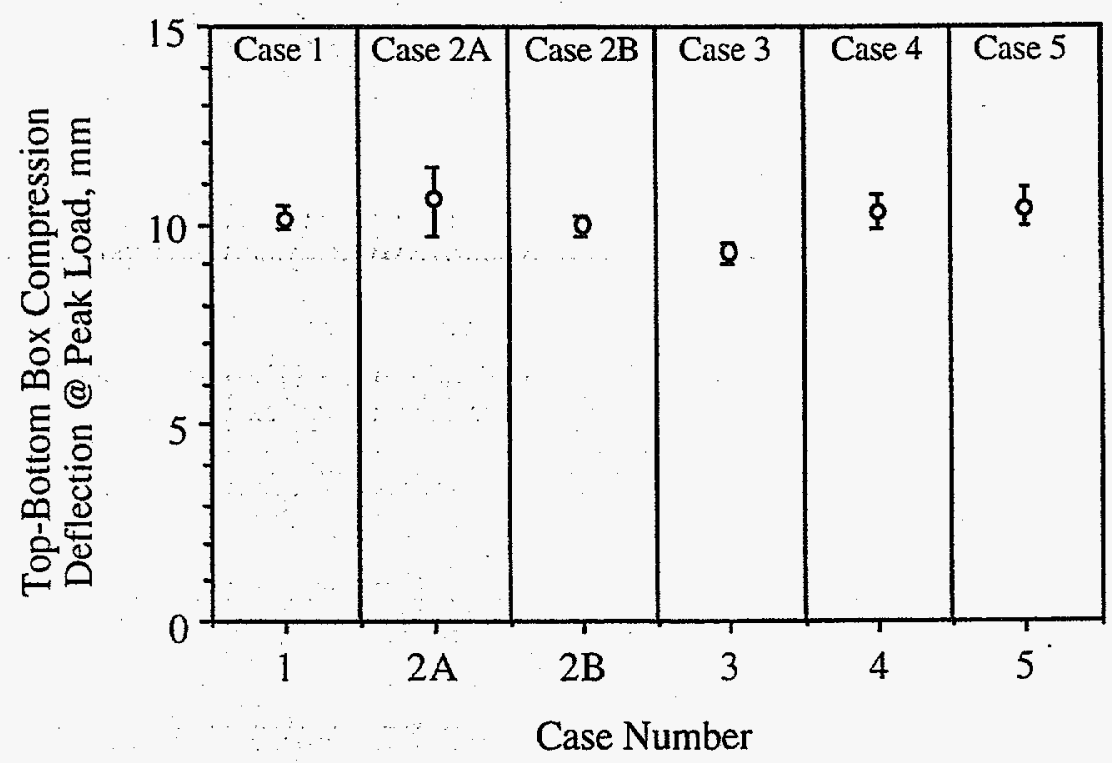

Figure C14. Top-To-Bottom Box Compression Deflection at Peak Load Plotted Versus 


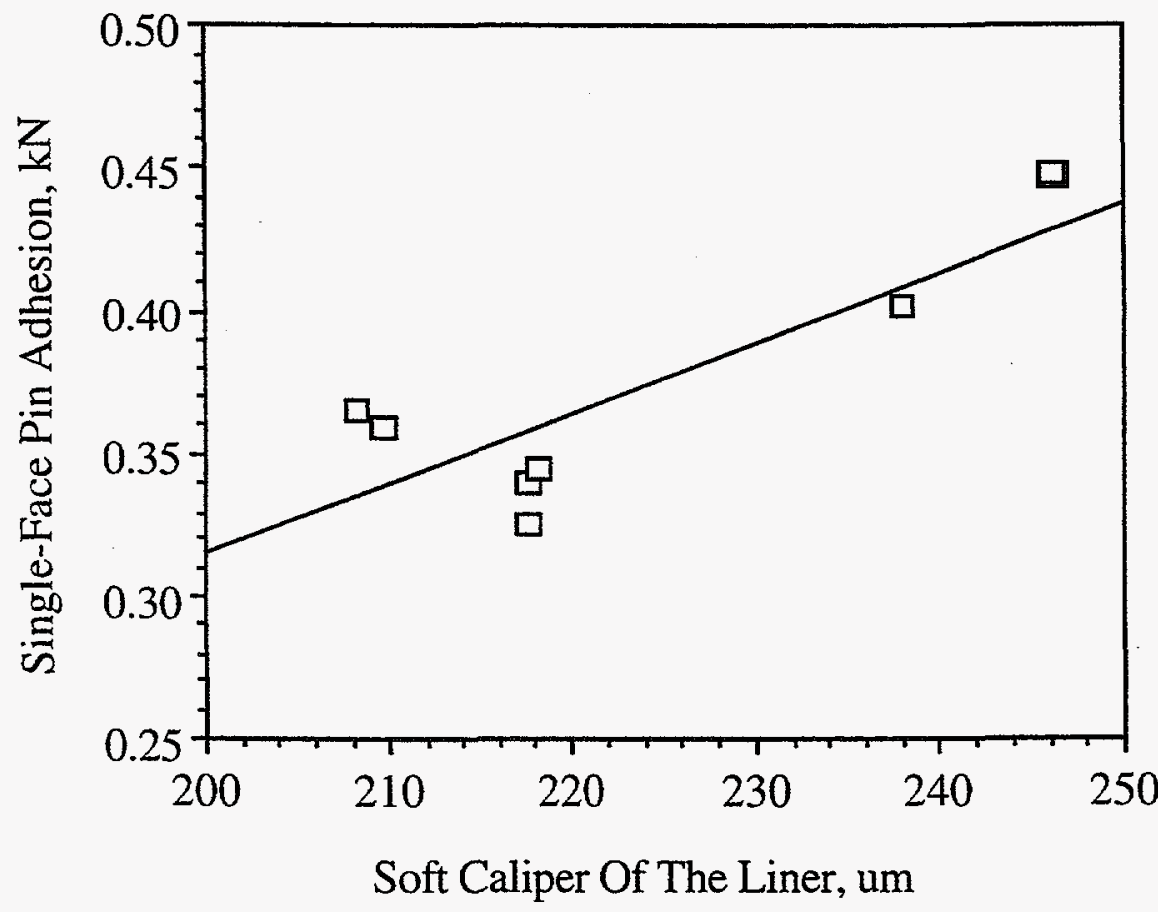

Figure C15. Single-Face Pin Adhesion Versus Linerboard Soft Caliper.

\section{ECONOMICS}

\section{D1.) Summary}

In September 1998 the Institute and Beloit Corporation were successful in producing 161 $\mathrm{g} / \mathrm{m}^{2}$ (33\#) linerboard on a one-meter wide pilot paper machine. The demonstration included a comparison of impulse drying to single-felted wet pressing.

Test results show that impulse drying, when compared to single-felted wet pressing, yielded a 4 point increase in press dryness, and a $10 \%$ increase in CD STFI and CD ring crush.

Reels of wet pressed and impulse dried linerboard, produced on a pilot paper machine, were converted on a Stone Container commercial corrugator in October of 1998.

Combined board and boxes made from the impulse dried linerboard were $10 \%$ stronger than board and boxes produced from the wet pressed linerboard.

Economic calculations demonstrate that impulse drying may be used to increase papermachine productivity, on a tonnage basis, by about $17.2 \%$ for dryer limited machines. On 
an area basis, basis weight reductions can be used to improve productivity by as much as $30 \%$ with substantial pulp cost savings.

\section{D2.) Assumptions}

It is assumed that an impulse dryer will be used to replace a third press on a paper machine that is currently dryer limited. It is further assumed that the installation of the impulse dryer will yield an improvement in press solids. The impuise dryer will also yield a strength improvement that will allow a basis weight reduction. The increased press dryness and basis weight reduction will allow the paper-machine speed to be increased until the machine is once again dryer limited.

For the wet pressing case, energy costs will include electric energy costs associated with pulp refining and steam costs associated with conventional drying. For the impulse drying case, the electric energy costs will include those for impulse dryer roll heating as well as pulp refining; steam costs for conventional drying will also be calculated.

As linerboard is sold on the basis of strength rather than by basis weight, the energy cost penalty of using impulse drying will be calculated on an area production basis. In a similar way, pulp cost savings will also be calculated on an area production basis.

\section{D3. Calculations}

\section{Wet Pressing}

Table D1 shows the input data for the conventional wet pressed case. In this example, the product at the reel is at $R \mathbf{S}=95 \%$ solids and the width at the reel is $\mathbf{W}=10 \mathrm{~m}$. The linerboard that is produced has an o.d. basis weight of OBWM $=150 \mathrm{~g} / \mathrm{m}^{2}$ (OBWF $=30.7$ $\left.\mathrm{lb} / 1000 \mathrm{ft}^{2}\right)$. The basis weight at the reel is calculated from the reel solids and o.d. basis weight as $\mathrm{RBWM}=158 \mathrm{~g} / \mathrm{m}^{2}$ (RBWF $\left.=32.3 \mathrm{lb} / 1000 \mathrm{ft}^{2}\right)$.

$$
\begin{aligned}
& \text { OBWF }=0.20482(\text { OBWM }) \\
& \text { RBWF }=100(\text { OBWF } /(\text { RS }) \\
& \text { RBWM }=100(\text { OBWM }) /(\text { RS })
\end{aligned}
$$

The structure of the sheet influences the average freeness, which impacts needed refining energy. Hence, the freeness and percent of total weight of each of two plies are given. For the case shown, the top ply represents $\% B W(1)=15 \%$ of the weight and is refined to $F(1)=669 \mathrm{ml} \mathrm{CSF}$, while the bottom ply, representing the remaining $\% B W(2)=$ $85 \%$ of the weight, is refined to a freeness of $F(2)=669 \mathrm{ml} \mathrm{CSF}$. The o.d. basis weight of each ply, $B W(i)$, is calculated from the weight percentages and the o.d. basis weight of the sheet.

$$
B W(i)=(\% B W(i))(O B W F) / 100
$$

The input and output conditions of the dryer section are given. In the example, solids into the dryer was $\% \operatorname{Sin}=47 \%$ and solids out of the dryer was $\%$ Sout $=95 \%$. As an estimate, the mass of steam used in drying was assumed to be $R=1.5$ times the mass of water that must be evaporated. The pressure of the steam in the conventional dryers was assumed to be $\mathbf{P}=7.74$ bar. Based on the steam tables, the latent heat of the steam was calculated as $\mathrm{Hfg}=2051 \mathrm{~kJ} / \mathrm{kg}$. 


$$
\mathrm{Hfg}=2240.2-30.61 \mathrm{P}+0.79767 \mathrm{P}^{2}
$$

At this point, the cost data for both the wet pressing and impulse drying cases are entered in Table 3-1. In the example, electricity costs C1 $=\$ 0.03 / \mathrm{kW}-\mathrm{hr}$, pulp costs $\mathbf{C 3}=$ $\$ 200 /$.d. ton and steam costs $\mathbf{C 2}=\$ 0.01 / \mathrm{kW}-\mathrm{hr}\left(\mathbf{C 2}^{\prime}=\$ 2.83 / \mathrm{million}^{\prime}\right.$ Btu $)$.

$$
\mathrm{C}^{\prime}=282.85(\mathrm{C} 2)
$$

The paper-machine speed was also entered. For the example, the dryer limited machine speed was taken as $S=600 \mathrm{~m} / \mathrm{min}$.

In Table D2 the water evaporated by the dryer section was calculated as We $=161$ $\mathrm{g} / \mathrm{m}^{2}(\mathrm{WE}=58,052 \mathrm{~kg} / \mathrm{hr})$.

$$
\begin{aligned}
& W e=[\{(1-(\% \operatorname{Sin} / 100)) /(\% \operatorname{Sin} / 100)\}-\{(1- \\
& (\% \text { Sout } / 100)) /(\% \text { Sout } / 100)\}](\text { OBWM }) \\
& W E=0.06 \text { We (S) (W) }
\end{aligned}
$$

The amount of steam used in the dryer section is calculated as $S U=87,077 \mathrm{~kg} / \mathrm{hr}$. Likewise, the steam energy is calculated as SEU $=178,601,144 \mathrm{~kJ} / \mathrm{hr}$. The steam energy per $\mathrm{kg}$ of product at the reel is calculated as SEUR $=0.873 \mathrm{~kW}-\mathrm{hr} / \mathrm{kg}$.

$$
\begin{aligned}
S U & =R(W E) \\
\text { SEU } & =H f g(\text { SU }) \\
\text { SEUR } & =0.0002778 \text { SEU/Pr }
\end{aligned}
$$

Where the amount of paper produced at the reel is $\mathrm{Pr}=56,842 \mathrm{~kg} / \mathrm{hr}$, or on an area basis, $\mathrm{PR}=360,000 \mathrm{~m}^{2} / \mathrm{hr}$.

$$
\begin{aligned}
\mathrm{Pr} & =0.06 \mathrm{~S}(\mathrm{~W})(\mathrm{RBWM}) \\
\mathrm{PR} & =1000(\mathrm{Pr}) /(\mathrm{RBWM})
\end{aligned}
$$

Also shown in Table 3-2 are the pulp usage, $\mathrm{PU}=0.0154 \mathrm{OD}$ ton $/ 1000 \mathrm{ft}^{2}$ and pulp costs, $\mathrm{CP}=\$ 3.07 / 1000 \mathrm{ft} 2$, at the reel.

$$
\begin{aligned}
& \mathrm{PU}=0.00010241 \text { (OBWM) } \\
& \mathrm{CP}=\mathrm{PU}(\mathrm{C} 3)
\end{aligned}
$$

In order to calculate energy used in refining, the average freeness was first calculated as Fave $=669 \mathrm{ml} \mathrm{CSF}$. The refining energy is then estimated as $R e=71 \mathrm{~kW}$-hr/ton at the reel $(\mathrm{RER}=0.079 \mathrm{~kW}-\mathrm{hr} / \mathrm{kg}$ at the reel) for the sample case.

$$
\begin{aligned}
\text { Fave } & =[(\% B W(1) / 100)(F(1))]+[(\% B W(2) / 100)(F(2))] \\
R e & =390.77-0.47733(\text { Fave }) \\
\text { RER } & =0.0011023(\mathrm{Re})
\end{aligned}
$$

The cost of energy may now be calculated. In particular, the total energy cost for the sample case was calculated as CTEAF $=\$ 0.16 / 1000 \mathrm{ft}^{2}$ of production at the reel.

$$
\mathrm{CEL}=\mathrm{RER}(\mathrm{C} 1)
$$


$\begin{array}{rlr}\text { CST } & =\text { SEUR }(\mathrm{C} 2) & \{20\} \\ \text { CTE }=\text { CEL }+ \text { CST } & \{21\} \\ \text { CTET }=907.186(\text { CTE }) & \{22\} \\ \text { CELA }=\text { CEL (RBWM)/1000 } & \{23\} \\ \text { CSTA }=\text { CST (RBWM)/1000 } & \{24\} \\ \text { CTEA }=\text { CELA + CSTA } & \{25\} \\ \text { CTEAF }=92.905(\text { CTEA }) & \{26\}\end{array}$

\section{Impulse Drying}

The impulse drying case, as shown in Table D3, assumes that the product basis weight can be reduced by $\%$ WR $=10 \%$ without sacrificing product strength. The o.d. basis weight can then be calculated as OBWMd $=135 \mathrm{~g} / \mathrm{m}^{2}\left(\right.$ OBWFd $\left.=27.7 \mathrm{lb} / 1000 \mathrm{ft}^{2}\right)$ and the basis weight at the reel as RBWMd $=142 \mathrm{~g} / \mathrm{m}^{2}$ (RBWFd $=29.1 \mathrm{lb} / 1000 \mathrm{ft}^{2}$ ).

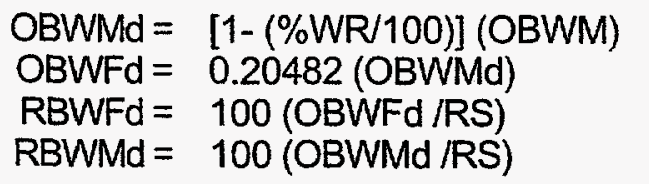

Reflecting the increased refining of our pilot experiments, the freeness of each of the layers of the sample case are assumed to be Fd (i) $=613 \mathrm{ml} \mathrm{CSF}$. Also, as in our pilot experiments, we have utilized an experimental correlation to predict press dryness of $\%$ Sind $=51 \%$, resulting from the impulse dryer operating at a press roll temperature of $\mathbf{T}$ $=260^{\circ} \mathrm{C}$.

$$
\% \text { Sind }=47.121-0.0086215 \mathrm{~T}+0.000087128 \mathrm{~T}^{2}
$$

Given the press solids performance of the impulse dryer, the water that must be evaporated in the dryer section can be calculated for the sample case as, Wed $=124$ $\mathrm{g} / \mathrm{m}^{2}$, see Table D4. Based on the water that can be evaporated by the "dryer-limited" dryer, and the reduction in basis weight of the product, the increased paper-machine speed may be calculated as $\mathbf{S d}=781 \mathrm{~m} / \mathrm{min}$.

$$
\begin{aligned}
\text { Wed }= & {[\{(1-(\% \text { Sind } / 100)) /(\% \text { Sind } / 100)\}-\{(1-} \\
& (\% \text { Soutd } / 100)) /(\% \text { Soutd } / 100)\}](\text { OBWMd }) \\
\text { Sd }= & 0.06 \text { WE } /(\text { Wed })(W)
\end{aligned}
$$

Given production at the reel of Prd $=66,633 \mathrm{~kg} / \mathrm{hr}\left(\mathrm{PRd}=468,900 \mathrm{~m}^{2} / \mathrm{hr}\right)$, steam usage may be calculated as SEURd $=0.745 \mathrm{~kW}-\mathrm{hr} / \mathrm{kg}$ at the reel.

$$
\begin{aligned}
\text { SEURd }= & 0.0002778 \text { SEU } /(\text { Prd }) \\
\text { Prd }= & 0.06 \mathrm{Sd}(\mathrm{W})(\mathrm{RBWMd}) \\
\text { PRd }= & 1000 \mathrm{Prd} /(\mathrm{RBWMd})
\end{aligned}
$$

Pulp usage may then be calculated as Pud $=0.0138$ o.d. ton $/ 1000 \mathrm{ft}^{2}$ and the cost of that pulp may be calculated as $\mathrm{CPd}=\$ 2.77 / 1000 \mathrm{ft}^{2}$.

$$
\text { Pud }=0.00010241(\text { OBWMd) }
$$


$\mathrm{CPd}=\operatorname{Pud}(\mathrm{C} 3)$

To calculate refining energy, the average freeness was then calculated as Faved $=613$ $\mathrm{ml} \mathrm{CSF}$. The refining energy may then be estimated as Red $=98 \mathrm{~kW}-\mathrm{hr} / \mathrm{ton}$ at the reel (RERd $=0.108 \mathrm{~kW}-\mathrm{hr} / \mathrm{kg}$ at the reel) for the sample case.

$$
\begin{aligned}
\text { Faved }= & {[(\% \mathrm{BWd}(1) / 100)(\mathrm{Fd}(1))]+[(\% \mathrm{BWd}(2) / 100)} \\
& (\mathrm{Fd}(2))] \\
\text { Red }= & 390.77-0.47733(\text { Faved }) \\
\text { RERd }= & 0.0011023(\text { Red })
\end{aligned}
$$

Based on experimental roll heating measurements, the energy transferred by the impulse dryer, including losses, was RETd $=97 \mathrm{~kJ} / \mathrm{m}^{2}$ when the press roll was heated to $T=260$ ${ }^{\circ} \mathrm{C}$. The roll heating power usage may then be calculated as RHPUd $=12,606 \mathrm{~kW}$-hr/hr. The roll heating power is then RHPAd $=0.0269 \mathrm{~kW}-\mathrm{hr} / \mathrm{m}^{2}(\mathrm{RHPMd}=0.189 \mathrm{~kW}-\mathrm{hr} / \mathrm{kg}$ at the reel).

$$
\begin{aligned}
\text { RETd } & =0.19116(-92.257+2.3021 \mathrm{~T}) \\
\text { RHPUd } & =0.000278 \text { RETd }(\mathrm{PRd}) \\
\text { RHPAd } & =0.000053(-92.257+2.3021 \mathrm{~T}) \\
\text { RHPMd } & =1000 \mathrm{RHPAd} /(\mathrm{RBWMd})
\end{aligned}
$$

The cost of energy may now be calculated. In particular the total energy cost for the sample case is calculated as CTEAFd $=\$ 0.22 / 1000 \mathrm{ft}^{2}$ of production at the reel.

$$
\begin{aligned}
\text { CELd } & =(\text { RERd + RHPMd })(C 1) \\
\text { CSTd } & =\text { SEURd }(\text { C2 }) \\
\text { CTEd } & =\text { CELd }+ \text { CSTd } \\
\text { CTETd } & =907.186(\text { CTEd }) \\
\text { CELAd } & =\text { CELd }(\text { RBWMd }) / 1000 \\
\text { CSTAd } & =\text { CSTd }(\text { RBWMd }) / 1000 \\
\text { CTEAd } & =\text { CELAd + CSTAd } \\
\text { CTEAFd } & =92.905(\text { CTEAd })
\end{aligned}
$$

For simplicity, all of the input and output data are summarized on Table D5. Also shown at the bottom of Table d5 are the calculations of energy cost penalty of $\$ 4.79 /$ ton at the reel $\left(\$ 0.05 / 1000 \mathrm{ft}^{2}\right)$, productivity increase of $17.2 \%$ on a tonnage basis and $30.2 \%$ on a product area basis. Pulp cost savings for the sample case was $\$ 0.31 / 1000 \mathrm{ft}^{2}$. Clearly, the benefit of saving pulp costs far outweighs slightly higher energy costs.

$$
\begin{aligned}
\text { Energy Cost Penalty (Tonnage) } & =\text { CTETd }- \text { CTET } \\
\text { Energy Cost Penalty (Area) } & =\text { CTEAFd }- \text { CTEAF } \\
\text { Productivity Increase (Tonnage) } & =100(\text { Prd-Pr)/(Pr) } \\
\text { Productivity Increase (Area) } & =100(\text { PRd-PR) } /(P R) \\
\text { Pulp Cost Savings (Area) } & =\text { CP-CPd }
\end{aligned}
$$

\section{D4.) Discussion}

The economic model described in the previous section was exercised to explore the impact of operating parameters (basis weight reduction, electric power cost, and pulp cost) on economic incentives of the technology. All of the other parameiers of the model were set to those of the sample case reviewed in the previous section. 
Figure D1 shows the impact of basis weight reduction and electric power costs on the energy penalty (on a tonnage basis) associated with using the technology. At a typical electric power cost of $\$ 0.03$ to $\$ 0.04$ per $\mathrm{kW}-\mathrm{hr}$, a cost penalty of between $\$ 5.00$ and $\$ 7.00$ per ton should be anticipated.

As linerboard is sold on strength properties and not basis weight, it is more relevant to explore the economics of the technology on the basis of cost per $1000 \mathrm{ft}^{2}$ of product produced. On that basis, Figure D2 shows that the energy cost penalty should range from $\$ 0.05$ to $\$ 0.08$ per $1000 \mathrm{ft}^{2}$ of production.

To put this energy cost penalty into perspective, we need to consider that reducing the basis weight will allow us to significantly increase productivity. This productivity increase is shown in Figure D3. It is observed that even without reducing the basis weight, impulse drying will result in a $17 \%$ increase in production. If we can reduce the basis weight by $10 \%$, we can increase productivity by about $30 \%$.

Our ability to reduce basis weight has a large impact on pulp costs. Figure D4 shows pulp cost savings as a function of pulp cost and the amount of basis weight reduction that we are able to achieve. Bracketing the pulp cost between $\$ 100$ to $\$ 200$ per ton and bracketing the basis weight reduction to between $7 \%$ and $10 \%$, we should anticipate pulp costs savings of between $\$ 0.10$ and $\$ 0.30$ per $1000 \mathrm{ft}^{2}$.

Combining the energy cost penalty with the pulp cost savings, impulse drying will positively impact operating costs from $\$ 0.02$ to $\$ 0.25$ per $1000 \mathrm{ft}^{2}$ of production. For a machine producing 121 millionft ${ }^{2} /$ day, operating cost savings of between $\$ 2,400$ to $\$ 30,000 /$ day could be realized.

\section{D5.) Acknowledgements}

The authors would like to thank the Member Companies of the Institute of Paper Science and Technology, the Beloit Corporation, and the U.S. Department of Energy (through Grant No. DOE/CE/40738) for supporting this research. The author would also like to thank David White of Union Camp Corporation for his contributions to this work. 
Table D1. Input Data: Conventional

\begin{tabular}{|c|c|c|}
\hline \multirow[t]{2}{*}{$\begin{array}{r}\text { Reel State: } \\
\text { (1a) }\end{array}$} & $\begin{array}{c}\text { Solids @ Reel } \\
\text { RS, \% }\end{array}$ & $\begin{array}{c}\text { Width @ Reel } \\
\text { W, m }\end{array}$ \\
\hline & 95 & 10 \\
\hline
\end{tabular}

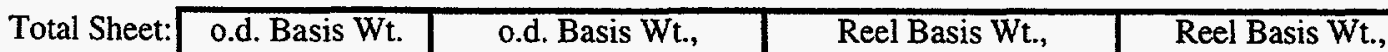

(1b) OBWM, g/m^2 OBWF, $\mathrm{lbm} / 1000 \mathrm{ft}^{\wedge} 2$

RBWF, $\mathrm{lbm} / 1000 \mathrm{ft}^{\wedge} 2$

RBWM, $\mathrm{g} / \mathrm{m}^{\wedge} 2$

150

30.7

32.3

158

\begin{tabular}{|c|c|c|c|c|}
\hline $\begin{array}{r}\text { Sheet Structure: } \\
\text { (1c) }\end{array}$ & $\begin{array}{l}\text { Ply Number } \\
\text { PLY(i) }\end{array}$ & $\begin{array}{c}\text { Freeness, } \\
\text { F(i), ml CSF }\end{array}$ & $\begin{array}{l}\text { OD Basis Wt., } \\
\text { \% BW(I), \% }\end{array}$ & $\begin{array}{c}\text { OD Basis Wt., } \\
\text { BW(i), } l b m / 1000 \mathrm{ft}^{\wedge} 2\end{array}$ \\
\hline & $\begin{array}{l}1 \\
2\end{array}$ & $\begin{array}{l}669 \\
669\end{array}$ & $\begin{array}{l}15 \\
85\end{array}$ & $\begin{array}{c}4.6 \\
26.1\end{array}$ \\
\hline
\end{tabular}

\begin{tabular}{|c|c|c|c|c|}
\hline $\begin{array}{l}\text { Drying Input Data: } \\
\text { (1d) }\end{array}$ & $\begin{array}{c}\text { Solids } \\
\text { Into Dryer } \\
\text { \% Sin, \% }\end{array}$ & $\begin{array}{c}\text { Solids } \\
\text { Out Of Dryer } \\
\text { \%Sout, \% }\end{array}$ & $\begin{array}{c}\text { Steam/Water } \\
\text { Use Ratio } \\
\text { R }\end{array}$ & $\begin{array}{c}\text { Steam Pressure } \\
\mathbf{P}, \text { bar }\end{array}$ \\
\hline & 47 & 95 & $\overline{1.5}$ & 7.74 \\
\hline \multirow[t]{2}{*}{$\begin{array}{r}\text { Cost Input Data: } \\
\text { (1e) }\end{array}$} & $\begin{array}{c}\text { Electricity } \\
\text { Cost } \\
\text { C1, \$/kW-hr }\end{array}$ & $\begin{array}{c}\text { Steam } \\
\text { Cost } \\
\text { C2, \$/kW-hr }\end{array}$ & $\begin{array}{c}\text { Steam } \\
\text { Cost } \\
\text { C2', } \$ / \text { million Btu }\end{array}$ & $\begin{array}{c}\text { Pulp } \\
\text { Cost } \\
\text { C3, \$/o.d.Ton }\end{array}$ \\
\hline & 0.030 & 0.01 & 2.83 & 200 \\
\hline
\end{tabular}

Dryer Limited Speed @ Reel

PM Conditions: $\quad$ S, $\mathrm{m} / \mathrm{min}$

(1f)




\begin{tabular}{|c|c|c|c|}
\hline \multirow{2}{*}{$\begin{array}{r}\text { Water Evaporated by } \\
\text { Dryer Section } \\
(2 \mathrm{a})\end{array}$} & $\begin{array}{c}\text { Water Evaporated } \\
\text { We, } g / \mathrm{m}^{\wedge} 2\end{array}$ & $\begin{array}{c}\text { Water Evaporated } \\
\text { WE, } \mathrm{kg} / \mathrm{hr}\end{array}$ & \\
\hline & 161 & 58052 & \\
\hline \multirow{2}{*}{$\begin{array}{r}\text { Steam Used By } \\
\text { Dryer Section: } \\
(\mathbf{2 b})\end{array}$} & $\begin{array}{l}\text { Steam Used } \\
\text { SU, kg/hr }\end{array}$ & $\begin{array}{l}\text { Steam Energy Used } \\
\text { SEU, kJ/hr }\end{array}$ & $\begin{array}{c}\text { Steam Energy Used } \\
\text { SEUR, kW-hr/kg @ Reel }\end{array}$ \\
\hline & 87077 & 178601144 & 0.873 \\
\hline
\end{tabular}

Paper Production Rate: Paper Produced @ Reel Paper Produced @ Reel

(2c)

$\frac{\operatorname{Pr}, \mathrm{kg} / \mathrm{h}}{56842}$

PR, $\mathrm{m}^{\wedge} 2 / \mathrm{hr}$

360000

Pulp Usage

PU, o.d.Ton $/ 1000 \mathrm{ft}^{\wedge} 2$

0.0154

Pulp Cost

$\mathrm{CP}, \$ / 1000 \mathrm{ft}^{\wedge} 2$

3.07

\begin{tabular}{|c|c|c|c|}
\hline \multirow[t]{2}{*}{$\begin{array}{r}\text { Refining Energy: } \\
\text { (2d) }\end{array}$} & $\begin{array}{c}\text { Average Freeness } \\
\text { Fave, ml CSF }\end{array}$ & $\begin{array}{l}\text { Refining Energy Use } \\
\text { Re, kW-hr/ton @ Reel }\end{array}$ & $\begin{array}{c}\text { Refining Energy Use } \\
\text { RER, kW-hr/kg @ Reel }\end{array}$ \\
\hline & 669 & 71 & 0.079 \\
\hline
\end{tabular}

Cost Of Energy:

(2e)

Cost Of Electric Power CEL, \$/kg@ Reel

Cost Of Steam CST, \$/kg@ Reel

Total Energy Cost CTE, \$/kg @ Reel 0.0087 0.0111

Total Energy Cost CTET, \$/ton @ Reel 0.0024

Cost Of Steam

Total Energy Cost CTEA, $\$ / \mathrm{m}^{\wedge} 2$ @ Reel

Total Energy Cost

Cost Of Energy: \begin{tabular}{l} 
Cost Of Electric Power \\
CELA, $\$ / \mathrm{m}^{\wedge} 2 @$ Reel \\
\hline 0.00037
\end{tabular} CSTA, \$/m^2@Reel 0.00138 0.0018 CTEAF, $\$ / 1000 \mathrm{ft}^{\wedge} 2 @$ Reel

Variable Costs: Variable Energy Costs VECOST, \$/day

Variable Pulp Costs VPCOST, \$day

Total Variable Costs 15,130 285,719 TVCOST, \$/day 10.06

(2g)

15,130

285,719


Table D3. Input Data: Impulse Drying

\begin{tabular}{|c|c|c|}
\hline \multirow[t]{2}{*}{$\begin{array}{r}\text { Reel State: } \\
(3 \mathbf{a})\end{array}$} & $\begin{array}{c}\text { Solids @ Reel } \\
\text { RS, \% }\end{array}$ & $\begin{array}{c}\text { Width @ Reel } \\
\text { W, m }\end{array}$ \\
\hline & 95 & 10 \\
\hline
\end{tabular}

(3b)

o.d. Wt. Reduction \%WR, \%

10

Total Sheet: o.d. Basis Wt.

(3c) OBWMd, $g / m^{\wedge} 2$

o.d. Basis Wt.,

Reel Basis Wt.,

Reel Basis Wt.,

OBWFd, $\mathrm{lbm} / 1000 \mathrm{ft}^{\wedge} 2$

RBWFd, $1 \mathrm{bm} / 1000 \mathrm{ft} \wedge 2$

RBWMd, $\mathrm{g} / \mathrm{m}^{\wedge} 2$

135

27.7

29.1

142

Sheet Structure:

\begin{tabular}{|c|}
\hline $\begin{array}{c}\text { Ply Number } \\
\text { PLY(i) }\end{array}$ \\
\hline 1 \\
2 \\
\hline
\end{tabular}

\begin{tabular}{c}
$\begin{array}{c}\text { Freeness, } \\
\text { Fo(i), ml CSF }\end{array}$ \\
\hline 613 \\
613 \\
\hline
\end{tabular}

\begin{tabular}{|c|}
\hline $\begin{array}{l}\text { o.d. Basis Wt., } \\
\% \text { BWd(i), \% }\end{array}$ \\
\hline $\begin{array}{l}15 \\
85\end{array}$ \\
\hline
\end{tabular}
BWd(i), $\mathrm{lbm} / 1000 \mathrm{ft}^{\wedge} 2$

4.1

23.5

Drying input Data:

(3e)

\begin{tabular}{|c|c|c|c|c|}
\hline $\begin{array}{c}\text { Solids } \\
\text { Into Dryer } \\
\% \text { Sind, \% }\end{array}$ & $\begin{array}{c}\text { Solids } \\
\text { Out Of Dryer } \\
\% \text { Sout, } \%\end{array}$ & $\begin{array}{c}\text { Steam/Water } \\
\text { Use Ratio } \\
\mathbf{R}\end{array}$ & $\begin{array}{c}\text { Steam Pressure } \\
\text { P, bar }\end{array}$ & $\begin{array}{c}\text { Latent Heat } \\
\text { Of Steam } \\
\mathbf{H f g}, \mathrm{kJ} / \mathrm{kg}\end{array}$ \\
\hline 51 & 95 & 1.5 & 7.74 & 2051 \\
\hline
\end{tabular}

Cost Input Data:

(3f)

\begin{tabular}{|c|c|c|c|}
\hline $\begin{array}{c}\text { Electricity } \\
\text { Cost } \\
\text { C1, } \$ / \mathrm{kW}-\mathrm{hr}\end{array}$ & $\begin{array}{c}\text { Steam } \\
\text { Cost } \\
\text { c2, } \$ / \mathrm{kW}-\mathrm{hr}\end{array}$ & $\begin{array}{c}\text { Steam } \\
\text { Cost } \\
\text { C2', \$/million Btu }\end{array}$ & $\begin{array}{c}\text { Pulp } \\
\text { Cost } \\
\text { C3, \$/o.d. Ton }\end{array}$ \\
\hline 0.030 & 0.01 & 2.83 & 200 \\
\hline
\end{tabular}

Impulse Drying Data: Roll Temperature

(3g) $\quad T,{ }^{\circ} \mathrm{C}$

260 
Table D4. Calculations: Impuise Drying

\begin{tabular}{r|c|} 
Water Evaporated & $\begin{array}{c}\text { Water Evaporated } \\
\text { Wed, } \mathrm{g} / \mathrm{m}^{\wedge} 2\end{array}$ \\
\hline By Dryer Section: & 124 \\
\hline
\end{tabular}

\begin{tabular}{r|r|}
$\begin{array}{r}\text { Speed @ Reel: } \\
\text { Table 4b }\end{array}$ & $\begin{array}{c}\text { Speed @ Reel } \\
\text { Sd, } \mathrm{m} / \mathrm{min}\end{array}$ \\
\hline 781 \\
\hline
\end{tabular}

\begin{tabular}{r|c|c|c|}
\cline { 2 - 4 } $\begin{array}{r}\text { Steam Used By } \\
\text { Dryer Section: }\end{array}$ & $\begin{array}{c}\text { Steam Used } \\
\text { SU, kg/hr }\end{array}$ & $\begin{array}{c}\text { Steam Energy Used } \\
\text { SEU, kJ/hr }\end{array}$ & $\begin{array}{c}\text { Steam Energy Used } \\
\text { SEURd, kW-hr/kg @ Reel }\end{array}$ \\
\cline { 2 - 4 } & 87077 & 178601144 & 0.745 \\
\hline
\end{tabular}

Paper Production Rate: Paper Produced @ Reel 1 Paper Produced @ Reel

(4d)

\begin{tabular}{|c|c|}
\hline $\begin{array}{c}\text { Paper Produced @ Reel } \\
\text { Prd, } \mathrm{kg} / \mathrm{hr}\end{array}$ & \\
\hline 66633 &
\end{tabular}
PRd, $m^{\wedge} 2 / h r$

Pulp Usage Pud, OD Ton/1000f^^2

Pulp Cost 66633 468900 0.0138

CPd, $\$ / 1000 \mathrm{ft}^{\wedge} 2$ 2.77

\begin{tabular}{r|c|c|c|} 
Refining Energy: & $\begin{array}{c}\text { Average Freeness } \\
\text { Faved, ml CSF }\end{array}$ & $\begin{array}{c}\text { Refining Energy Use } \\
\text { Red, kW-hr/Ton @ Reel }\end{array}$ & $\begin{array}{c}\text { Refining Energy Use } \\
\text { RERd, kW-hr/kg @ Reel }\end{array}$ \\
\hline 613 & 98 & 0.108 \\
\hline
\end{tabular}

1.D. Energy:

Poil Energy Transfer
RETd, kJ/m^2

Roll Heating Power Use RHPUd, kW-hr/hr

97 12606 Roll Heating Power
RHPAd kW-hr/m^?

Roll Heating Power RHPMd, kW-hr/kg @ Reel

\begin{tabular}{l|l}
0.0269 & 0.189
\end{tabular}

Cost Of Energy:

\begin{tabular}{|c|}
\hline $\begin{array}{c}\text { Cost Of Electric Power } \\
\text { CELd, } \$ / \mathrm{kg} @ \text { Reel }\end{array}$ \\
\hline 0.0089
\end{tabular}

Cost Of Steam CSTd, \$/ kg@Reel

0.0074

\begin{tabular}{|c|}
\hline $\begin{array}{c}\text { Total Energy Cost } \\
\text { CTEd, } \$ / \mathrm{kg} @ \text { Reel }\end{array}$ \\
\hline $\mathbf{0 . 0 1 6 4}$
\end{tabular}

Total Energy Cost CTETd, \$/ Ton @ Reel

0.0089

Cost Of Energy:

Cost Of Electric Power
CELAd, $\$ / m^{\wedge} 2 @$ Reel

Cost Of Steam CSTAd, \$/m^2 @ Reel

\begin{tabular}{|c} 
Total Energy Cost \\
CTEAd, $\$ / \mathrm{m}^{\wedge} 2$ @ Reel
\end{tabular}

Total Energy Cost 0.0013

0.0011
0.0023 CTEAFd, $\$ / 1000 \mathrm{ft}^{\wedge} 2$ @ Reel

Variable Costs:

Variable Energy Costs
VECOSTd, $\$$ day

\begin{tabular}{|r|r|}
\hline $\begin{array}{l}\text { Variable Pulp Costs } \\
\text { VPCOSTd, \$/day }\end{array}$ & \\
\hline 334,934 &
\end{tabular}

Total Variable Costs TVcostd, \$/day 


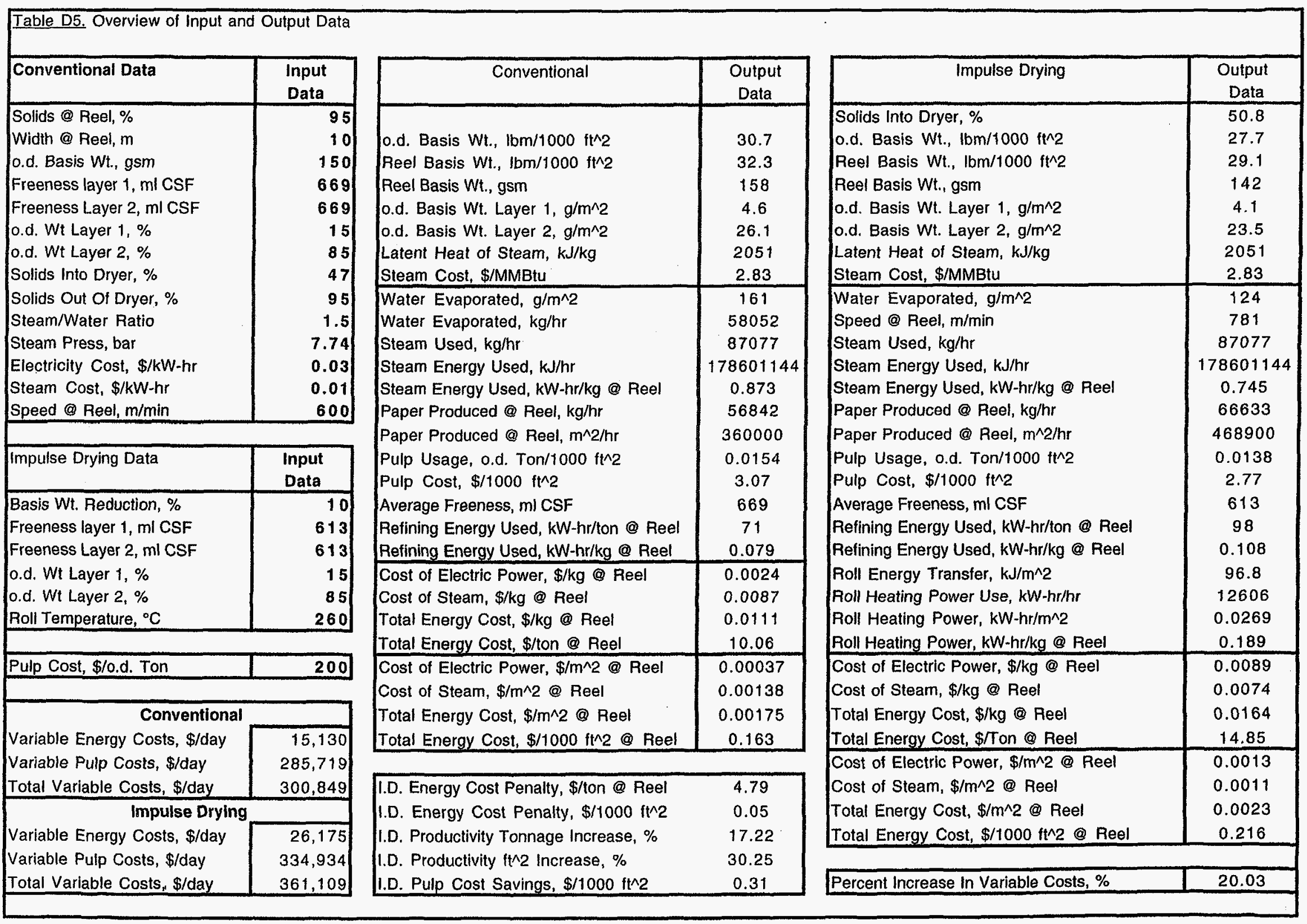




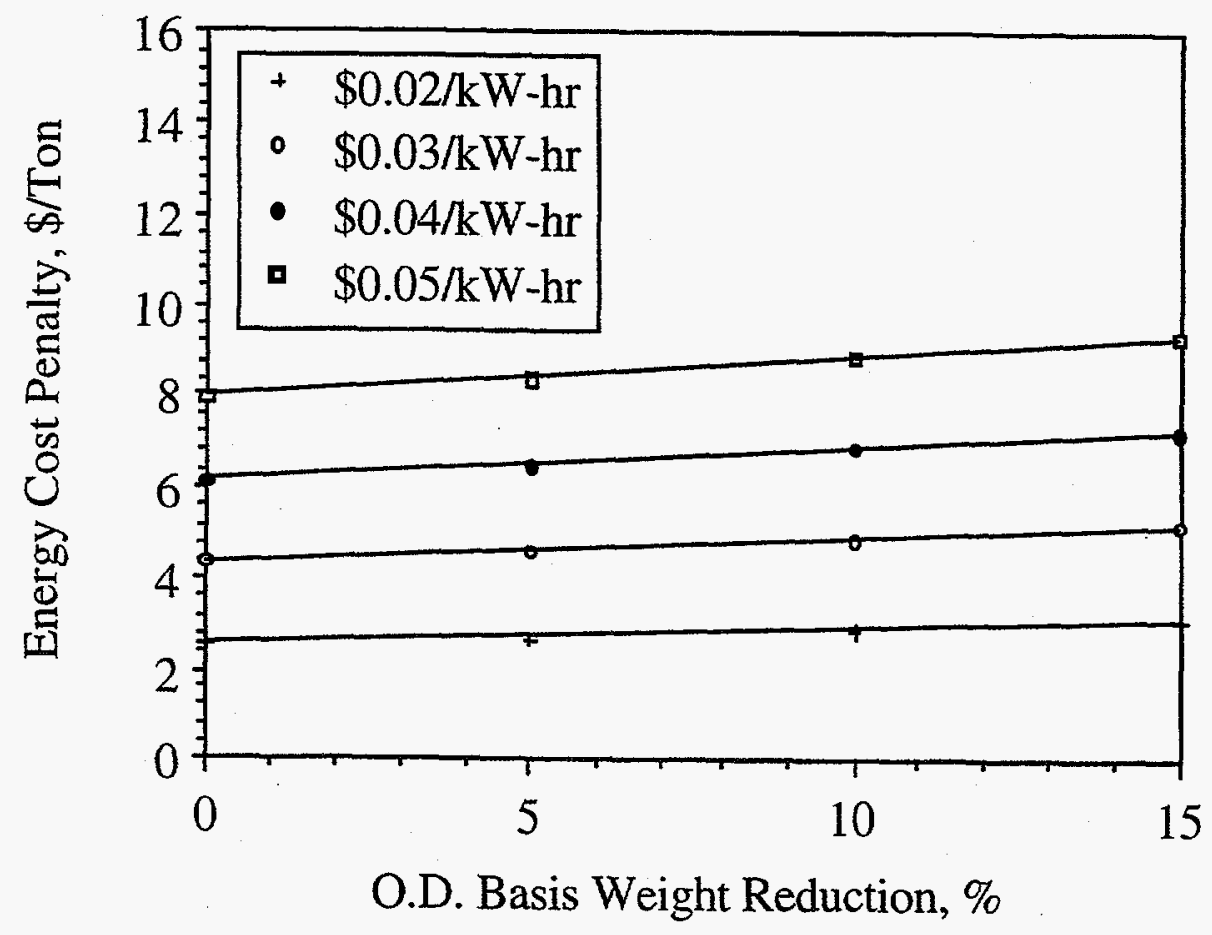

Figure D1. Energy Cost Penalty (Tonnage Basis)Versus O. D. Basis Weight Reduction. 


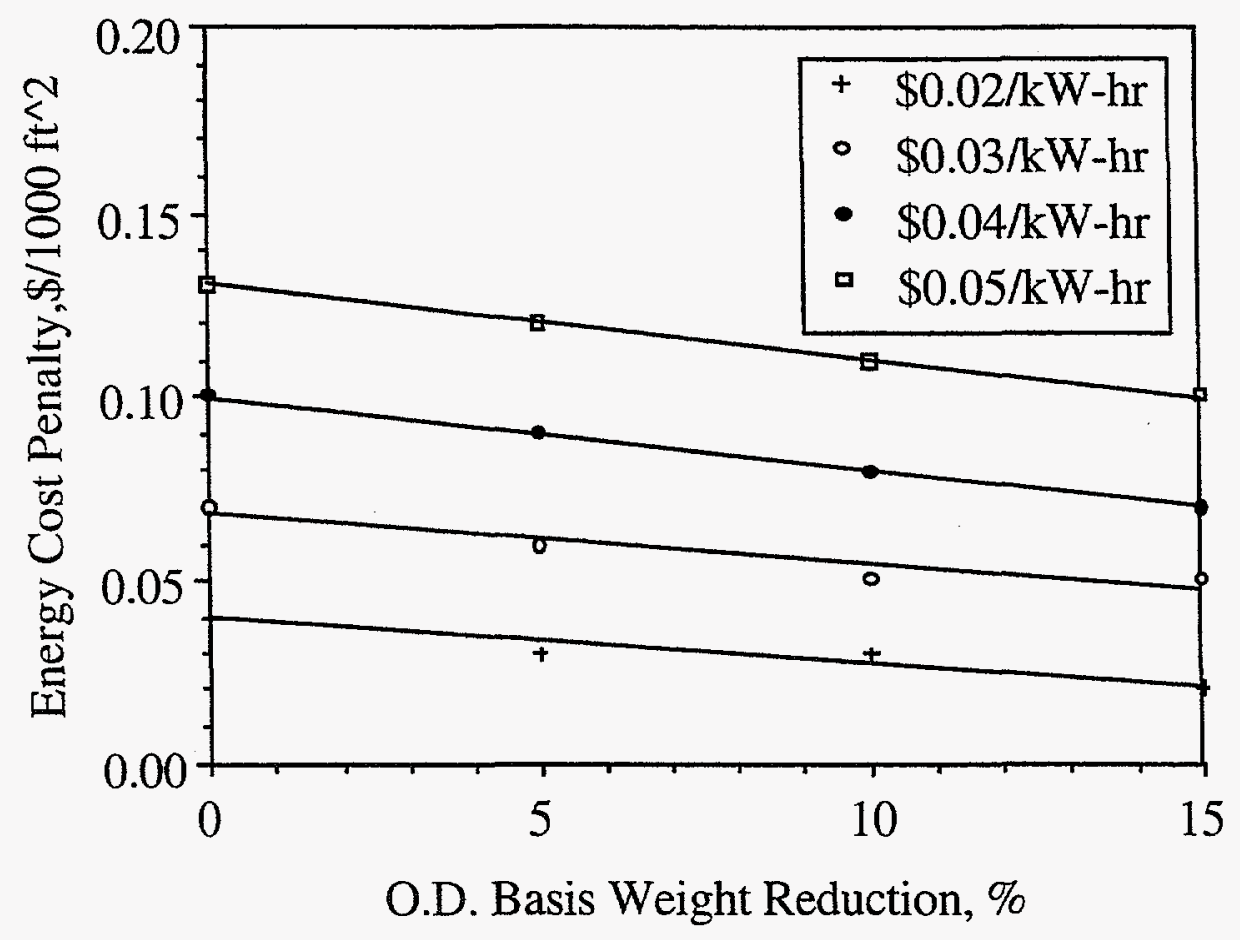

Figure D2. Energy Cost Penalty (Area Basis) Versus O.D. Basis Weight Reduction. 


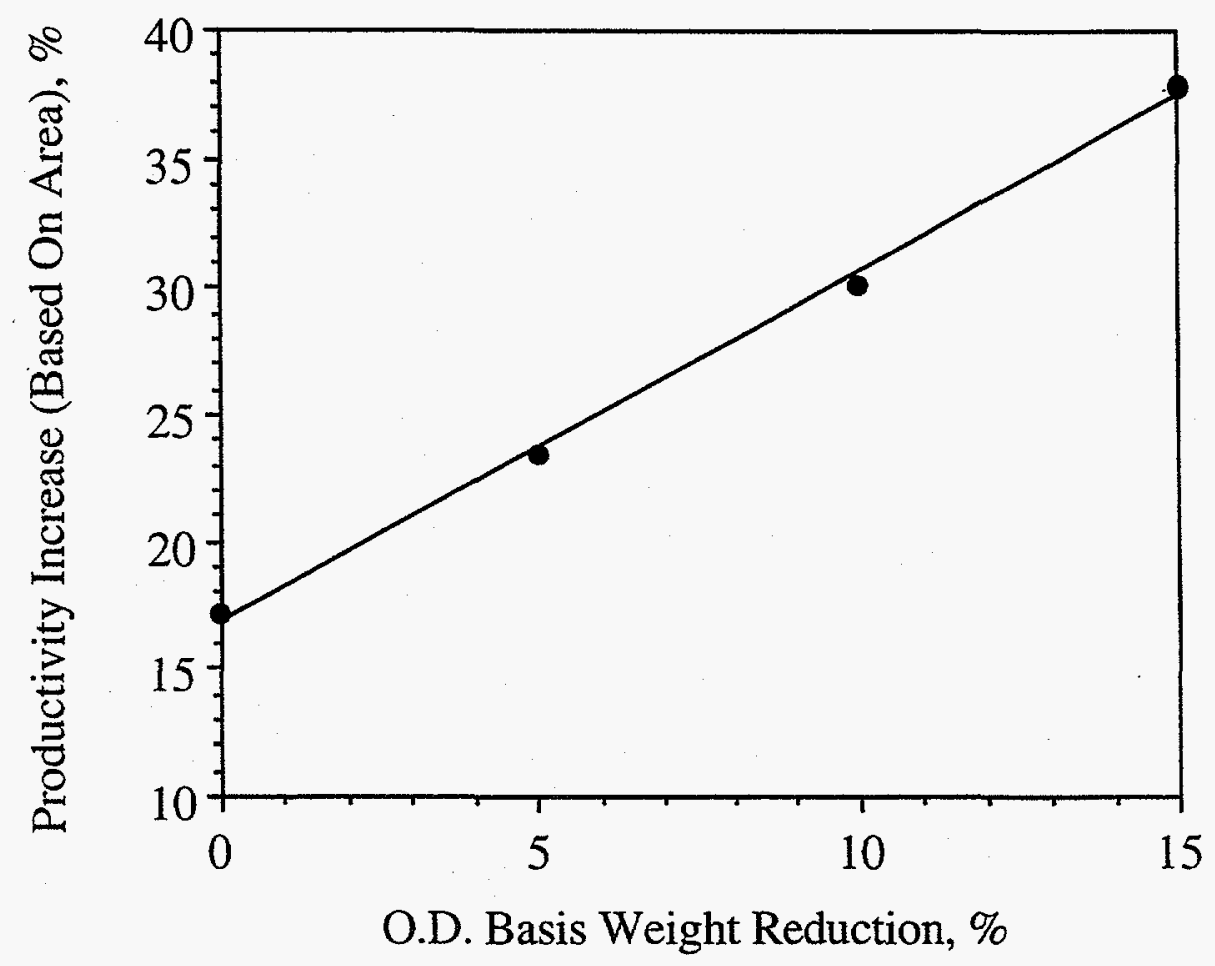

Fiqure D3. Productivity Increase (Area Basis) Versus O. D. Basis Weight Reduction. 


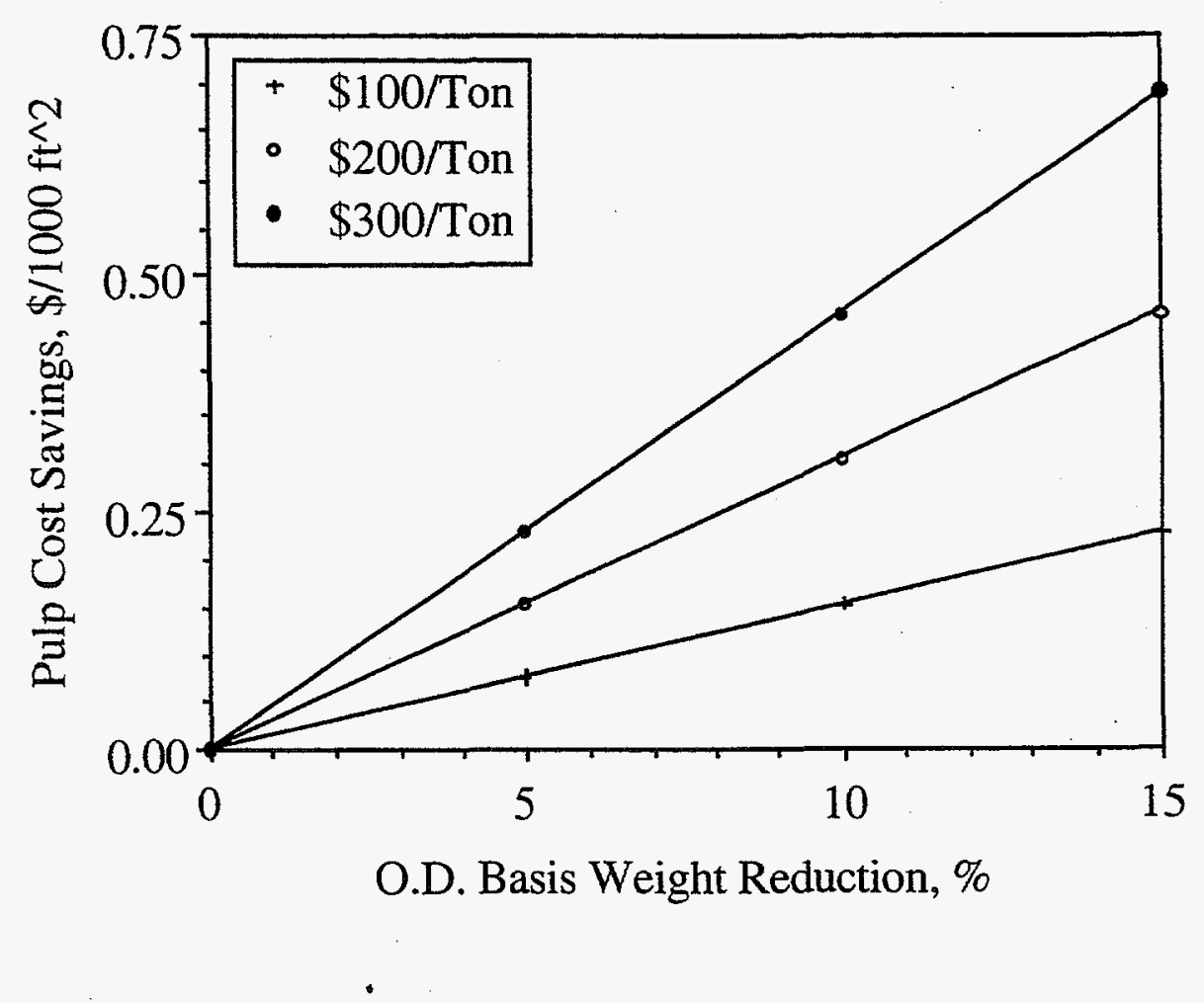

Figure D4. Pulp Cost Savings (Area Basis)Versus O. D. Basis Weight Reduction. 


\section{E. PAPER, BOARD AND BOX SAMPLING AND TESTING}

\section{E1.) Paper Sampling and Testing}

The final reel width of the liner was about $0.79 \mathrm{~m}$ (31 in), which was trimmed to $0.76 \mathrm{~m}$ (30 in) and rewound. Before trimming, full width samples from the outside of each production rolls and at the marked positions of the test rolls were cut and divided between Beloit and IPST for testing. For detailed testing at IPST, the liner was divided into three 10-inch wide lanes in the cross direction, designated as Operator (front), Center, and Drive (back) lanes. Depending on the physical test, one to four repeat samples at different machine direction positions were tested to decrease the error bars.

Test Indexes (STFI, ring crush, tensile) were calculated individually using the OD basis weight of each test strip. The average basis weight for each sample was the average of all of the oven dried strip basis weights. The density was calculated from the average OD basis weight and average soft platen caliper for each sample.

\section{E2.) Board and Box Sampling and Testing}

Two sizes of blanks were produced. For the boxes about 750 blanks of each case 1.52 $\mathrm{m}$ ( 60 inches) long by $0.69 \mathrm{~m}$ ( 27 inches) wide were made. The die-cut blanks were slightly smaller, $1.02 \mathrm{~m}$ ( 40 inches) long by $0.71 \mathrm{~m}$ (28 inches) wide and about 250 were made for each case. Only die-cut blanks (about 750) were produced for Case 6 resulting in fewer total samples. About 250 Case 6 blanks were run through the flexo/folder/gluer operation for printing only.

Boxes and die cut samples were selected from each case in four sets of ten samples representing each quarter of the corrugator run. Of these, one set was die-cut samples and three sets were boxes. One specimen for each type of test was cut out of each box/panel resulting in ten test repeats for each set of samples. Of course the box compresion and glue joint tests were only done on three sets of samples for each case (no tests for Case 6 since there were no boxes made). 
Table E1. Preliminary Data from Beloit Corporation.

\begin{tabular}{|c|c|c|c|c|c|c|c|c|c|}
\hline & $\begin{array}{l}\text { Sample } \\
\text { ID } \\
(\#)\end{array}$ & $\begin{array}{c}\text { Outgoing } \\
\text { Solids } \\
(\%)\end{array}$ & $\begin{array}{c}\text { Grammage } \\
\text { (Conditioned) } \\
\left(\mathrm{g} / \mathrm{m}^{\wedge} 2\right)\end{array}$ & $\begin{array}{c}\text { Basis } \\
\text { Weight } \\
\text { (Conditioned) } \\
\left(\mathrm{lb} / 1000 \mathrm{ft}^{\wedge} 2\right)\end{array}$ & $\begin{array}{c}\text { Apparent } \\
\text { Density } \\
\text { (Conditioned) } \\
(\mathrm{g} / \mathrm{cm} 3)\end{array}$ & $\begin{array}{c}\text { Scott } \\
\text { Bond } \\
(\mathrm{kJ} / \mathrm{m} 2)\end{array}$ & $\begin{array}{l}\text { MD STFI } \\
\text { Index } \\
\left(N^{*} \mathrm{~m} / \mathrm{g}\right)\end{array}$ & $\begin{array}{l}\text { CD STFI } \\
\text { Index } \\
\left(N^{*} \mathrm{~m} / \mathrm{g}\right)\end{array}$ & $\begin{array}{l}\text { CD STFI } \\
\text { (lbf/in) }\end{array}$ \\
\hline Case 5 & 11942 & 46.91 & 162.2 & 33.23 & 0.571 & 0.153 & 29.10 & 15.70 & 14.54 \\
\hline Wet Press & 11943 & 46.91 & 159.4 & 32.66 & 0.566 & 0.147 & 31.80 & 15.50 & 14.11 \\
\hline \multirow[t]{3}{*}{ No Calender } & 11944 & 46.91 & 165.3 & 33.87 & 0.581 & 0.141 & 28.30 & 15.10 & 14.25 \\
\hline & 11945 & 46.91 & 163.9 & 33.58 & 0.640 & 0.139 & 28.80 & 15.00 & 14.04 \\
\hline & Average & 46.91 & 162.7 & 33.34 & 0.590 & 0.145 & 29.50 & 15.33 & 14.24 \\
\hline Case 3 & 11946 & 46.91 & 156.2 & 32.00 & 0.608 & 0.130 & 30.70 & 15.60 & 13.91 \\
\hline Wet Press & 11947 & 46.91 & 160.9 & 32.97 & 0.603 & 0.141 & 31.00 & 16.50 & 15.16 \\
\hline \multirow[t]{3}{*}{$35 \mathrm{KN} / \mathrm{m}$ Calender } & 11948 & 46.91 & 159.9 & 32.76 & 0.606 & 0.139 & 31.20 & 14.90 & 13.60 \\
\hline & 11949 & 46.91 & 158.1 & 32.39 & 0.598 & 0.141 & 29.60 & 14.90 & 13.45 \\
\hline & Average & 46.91 & 158.8 & 32.53 & 0.604 & 0.138 & 30.63 & 15.48 & 14.03 \\
\hline Case 2 & 11926 & 49.47 & 158.8 & 32.54 & 0.615 & 0.168 & 33.69 & 18.60 & 16.87 \\
\hline $246{ }^{\circ} \mathrm{C}$ Roll Temp & 11930 & 49.47 & 163.0 & 33.40 & 0.640 & 0.174 & 33.14 & 17.89 & 16.65 \\
\hline \multirow[t]{3}{*}{ No Calender } & 11931 & 49.47 & 160.6 & 32.90 & 0.627 & 0.170 & 34.96 & 17.86 & 16.38 \\
\hline & 11932 & 49.47 & 158.7 & 32.52 & 0.648 & 0.177 & 34.27 & 18.29 & 16.57 \\
\hline & Average & 49.47 & 160.3 & 32.84 & 0.633 & 0.172 & 34.02 & 18.16 & 16.62 \\
\hline Case 6 & 11927 & 49.47 & 157.1 & 32.19 & 0.670 & 0.164 & 34.60 & 17.67 & 15.85 \\
\hline \multirow{2}{*}{$\begin{array}{l}246^{\circ} \mathrm{C} \text { Roll Temp } \\
35 \mathrm{KN} / \mathrm{m} \text { Calender }\end{array}$} & 11928 & 49.47 & 159.6 & 32.70 & 0.680 & 0.177 & 35.40 & 18.41 & 16.78 \\
\hline & Average & 49.47 & 158.4 & 32.44 & 0.675 & 0.171 & 35.00 & 18.04 & 16.31 \\
\hline Case 4 & 11933 & 50.47 & 156.1 & 31.98 & 0.611 & 0.183 & 35.31 & 16.42 & 14.64 \\
\hline $260^{\circ} \mathrm{C}$ Roll Temp & 11934 & 50.47 & 160.1 & 32.80 & 0.616 & 0.177 & 33.49 & 17.60 & 16.09 \\
\hline \multirow[t]{3}{*}{ No Calender } & 11935 & 50.47 & 161.3 & 33.05 & 0.638 & 0.177 & 34.78 & 17.81 & 16.40 \\
\hline & 11936 & 50.47 & 160.9 & 32.97 & 0.624 & 0.164 & 31.68 & 16.62 & 15.27 \\
\hline & Average & 50.47 & 159.6 & 32.70 & 0.622 & 0.175 & 33.82 & 17.11 & 15.60 \\
\hline $204^{\circ} \mathrm{C}$ Roll Temp & 11950 & 49.08 & 162.3 & 33.25 & 0.634 & 0.153 & 31.81 & 16.73 & 15.50 \\
\hline $218^{\circ} \mathrm{C}$ Roll Temp & 11951 & 49.35 & 159.8 & 32.74 & 0.634 & 0.156 & 33.12 & 16.94 & 15.46 \\
\hline $232^{\circ} \mathrm{C}$ Roll Temp & 11952 & 49.79 & 159.5 & 32.68 & 0.626 & 0.153 & 32.13 & 17.54 & 15.97 \\
\hline $246^{\circ} \mathrm{C}$ Roll Temp & 11953 & 50.38 & 161.2 & 33.03 & 0.627 & 0.160 & 30.28 & 17.86 & 16.44 \\
\hline $260^{\circ} \mathrm{C}$ Roll Temp & 11954 & 51.06 & 164.1 & 33.62 & 0.640 & 0.149 & 32.75 & 16.63 & 15.58 \\
\hline $274^{\circ} \mathrm{C}$ Roll Temp & 11955 & 51.06 & 164.1 & 33.62 & 0.640 & 0.149 & 32.75 & 16.63 & 15.58 \\
\hline $288^{\circ} \mathrm{C}$ Roll Temp & 11956 & 52.05 & 162.7 & 33.34 & 0.630 & 0.162 & 30.05 & 15.73 & 14.61 \\
\hline $\mathrm{WP}-43.8 \mathrm{KN} / \mathrm{m}$ Cal. & 11937 & & 162.7 & 33.34 & 0.637 & 0.145 & 30.1 & 16.0 & 14.87 \\
\hline WP - $37.1 \mathrm{KN} / \mathrm{m}$ Cal. & 11938 & & 161.4 & 33.07 & 0.628 & 0.143 & 29.5 & 17.2 & 15.85 \\
\hline WP - $10.5 \mathrm{KN} / \mathrm{m} \mathrm{Cal}$ & 11939 & & 161.5 & 33.09 & 0.588 & 0.145 & 30.8 & 16.3 & 15.03 \\
\hline WP - $28.7 \mathrm{KN} / \mathrm{m}$ Cal. & 11940 & & 159.6 & 32.70 & 0.585 & 0.143 & 31.3 & 15.9 & 14.49 \\
\hline WP - $19.8 \mathrm{KN} / \mathrm{m}$ Cal. & 11941 & & 158.0 & 32.37 & 0.570 & 0.147 & 31.6 & 16.1 & 14.53 \\
\hline
\end{tabular}


Table E1 cont. Preliminary Data from Beloit Corporation.

\begin{tabular}{|c|c|c|c|c|c|c|c|c|c|}
\hline & $\begin{array}{l}\text { Sample } \\
\text { ID } \\
(\#)\end{array}$ & $\begin{array}{l}\text { FS Bendtsen } \\
\text { Smoothness } \\
(\mathrm{ml} / \mathrm{min})\end{array}$ & $\begin{array}{l}\text { WS Bendtsen } \\
\text { Smoothness } \\
(\mathrm{ml} / \mathrm{min})\end{array}$ & $\begin{array}{l}\text { FS Sheffield } \\
\text { Smoothness } \\
\text { (SU) }\end{array}$ & $\begin{array}{l}\text { WS Sheffield } \\
\text { Smoothness } \\
\text { (SU) }\end{array}$ & $\begin{array}{c}\text { Gurley } \\
\text { Porosity } \\
\text { (s/100 ml) }\end{array}$ & $\begin{array}{c}\text { Bendtsen } \\
\text { Permeability } \\
(\mathrm{ml} / \mathrm{min})\end{array}$ & $\begin{array}{l}\text { MD Ring } \\
\text { Crush } \\
\text { Index } \\
(\mathrm{N} \mathrm{m} / \mathrm{g})\end{array}$ & $\begin{array}{l}\text { CD Ring } \\
\text { Crush } \\
\text { Index } \\
(\mathrm{N} \mathrm{m} / \mathrm{g})\end{array}$ \\
\hline Case 5 & 11942 & 2000 & 1985 & $>386$ & $>386$ & 9.8 & 1210 & 11.9 & 9.4 \\
\hline Wet Press & 11943 & 1935 & 1985 & $>386$ & $>386$ & 9.9 & 1210 & 11.9 & 8.6 . \\
\hline \multirow[t]{3}{*}{ No Calender } & $1: 944$ & 2020 & 2060 & $>386$ & $>386$ & 10.3 & 1175 & 12.0 & 8.6 \\
\hline & 11945 & 650 & 695 & 365 & 364 & 14.0 & 860 & 11.9 & 7.4 \\
\hline & Average & 1985 & 2010 & & & 11.0 & 1114 & 11.9 & 8.5 \\
\hline Case 3 & 11946 & 1370 & 1245 & $>383$ & $>380$ & 10.2 & 1175 & 11.7 & 8.4 \\
\hline Wet Press & 11947 & 1410 & 1380 & $>378$ & 377 & 9.8 & 1180 & 11.7 & 8.3 \\
\hline \multirow[t]{3}{*}{$35 \mathrm{KN} / \mathrm{m}$ Calender } & 11948 & 1400 & 1420 & $>385$ & $>382$ & 10.3 & 1185 & 10.2 & 6.8 \\
\hline & 11949 & 1290 & 1345 & $>385$ & $>386$ & 9.9 & 1185 & 11.4 & 8.9 \\
\hline & Average & 1393 & 1348 & & & 10.1 & 1181 & 11.3 & 8.1 \\
\hline Case 2 & 11926 & 1650 & 2090 & 384 & $>386$ & 24.5 & & $\overline{12.7}$ & 9.0 \\
\hline $246^{\circ} \mathrm{C}$ Roll Temp & 11930 & 1500 & 2240 & $>386$ & $>386$ & 24.9 & & 14.1 & 9.0 \\
\hline \multirow[t]{3}{*}{ No Calender } & 11931 & 1380 & 2130 & $>386$ & $>386$ & 26.2 & & 13.7 & 9.0 \\
\hline & 11932 & 1500 & 1920 & $>386$ & $>386$ & 25.9 & & 13.5 & 9.1 \\
\hline & Average & 1510 & 2153 & & & 25.4 & & 13.5 & 9.0 \\
\hline Case 6 & 11927 & 1110 & 1370 & 322 & 385 & 26.4 & & 11.5 & 9.5 \\
\hline $246^{\circ} \mathrm{C}$ Roll Temp & 11928 & 910 & 1450 & 331 & 380 & 26.6 & & 13.4 & 7.5 \\
\hline $35 \mathrm{KN} / \mathrm{m}$ Calender & Average & 1010 & 1410 & 326.5 & 382.5 & 26.5 & & 12.5 & 8.5 \\
\hline Case 4 & 11933 & 1330 & 2180 & $>386$ & $>386$ & 24.7 & & 12.8 & 10.3 \\
\hline $260^{\circ} \mathrm{C}$ Roll Temp & 11934 & 1430 & 1830 & $>386$ & $>386$ & 23.8 & & 12.2 & 8.8 \\
\hline \multirow[t]{3}{*}{ No Calender } & 11935 & 1270 & 2290 & $>386$ & $>386$ & 24.2 & & 14.0 & 10.1 \\
\hline & 11936 & 1320 & 2110 & $>386$ & $>386$ & 25.5 & & 13.1 & 9.5 \\
\hline & Average & 1343 & 2100 & & & 24.6 & & 13.0 & 9.7 \\
\hline $204^{\circ} \mathrm{C}$ Roll Temp & 11950 & 1685 & 2020 & $>386$ & $>386$ & 16.4 & 745 & 11.3 & 8.1 \\
\hline $218^{\circ} \mathrm{C}$ Roll Temp & 11951 & 1690 & 2060 & $>386$ & $>386$ & 17.3 & 715 & 12.4 & 10.4 \\
\hline $232^{\circ} \mathrm{C}$ Roll Temp & 11952 & 1525 & 2095 & $>386$ & $>386$ & 19.1 & 615 & 12.8 & 8.0 \\
\hline $246^{\circ} \mathrm{C}$ Roll Temp & 11953 & 1340 & 2055 & $>386$ & $>386$ & 18.3 & 640 & 12.7 & 9.1 \\
\hline $260^{\circ} \mathrm{C}$ Roll Temp & 11954 & 1560 & 2110 & $>386$ & $>386$ & 17.1 & 655 & 11.7 & 9.3 \\
\hline $274^{\circ} \mathrm{C}$ Roll Temp & 11955 & 1560 & 2110 & $>386$ & $>386$ & 17.1 & 690 & 11.1 & 8.7 \\
\hline $288^{\circ} \mathrm{C}$ Roll Temp & 11956 & 1530 & 2165 & $>386$ & $>386$ & 19.0 & 635 & 11.7 & 7.3 \\
\hline WP - $43.8 \mathrm{KN} / \mathrm{m}$ Cal. & 11937 & 1160 & 1150 & 362 & 370 & 12.7 & 955 & 11.1 & 7.5 \\
\hline WP $-37.1 \mathrm{KN} / \mathrm{m}$ Cal. & 11938 & 1265 & 1355 & 372 & $>383$ & 12.2 & 1025 & 11.3 & 8.4 \\
\hline WP $-10.5 \mathrm{KN} / \mathrm{m}$ Cal. & 11939 & 1805 & 1840 & $>386$ & $>386$ & 10.7 & 1140 & 12.4 & 9.2 \\
\hline WP - $28.7 \mathrm{KN} / \mathrm{m}$ Cal. & 11940 & 1900 & 1810 & $>386$ & $>386$ & 10.9 & 1105 & 13.3 & 10.0 \\
\hline WP - $19.8 \mathrm{KN} / \mathrm{m} \mathrm{Cal}$. & 11941 & 1925 & 1850 & $>386$ & $>386$ & 10.8 & 1135 & 12.1 & 8.3 \\
\hline
\end{tabular}


Table E1 cont. Preliminary Data from Beloit Corporation.

\begin{tabular}{|c|c|c|c|c|c|}
\hline & $\begin{array}{c}\text { Sample } \\
\text { ID } \\
(\#) \\
\end{array}$ & $\begin{array}{c}\text { Burst } \\
\text { Index } \\
\left(\mathrm{kPa} \mathrm{m}^{\wedge} 2 / \mathrm{g}\right)\end{array}$ & $\begin{array}{c}\text { MD } \\
\text { Tensile } \\
\text { Index } \\
(\mathrm{N} \mathrm{m} / \mathrm{g})\end{array}$ & $\begin{array}{c}\mathrm{CD} \\
\text { Tensile } \\
\text { Index } \\
(\mathrm{N} \mathrm{m} / \mathrm{g})\end{array}$ & $\begin{array}{c}\text { Tensile } \\
\text { Ratio }\end{array}$ \\
\hline Case 5 & 11942 & 2.59 & 66.65 & 24.08 & 2.77 \\
\hline Wet Press & 11943 & 2.32 & 66.00 & 23.78 & 2.77 \\
\hline \multirow[t]{3}{*}{ No Calender } & 11944 & 3.10 & 67.22 & 25.08 & 2.68 \\
\hline & 11945 & 2.82 & 67.67 & 25.50 & 2.65 \\
\hline & Average & 2.71 & 66.89 & 24.61 & 2.72 \\
\hline Case 3 & 11946 & 2.47 & 68.45 & 24.11 & 2.84 \\
\hline Wet Press & 11947 & 2.89 & 70.10 & 24.98 & 2.81 \\
\hline \multirow[t]{3}{*}{$35 \mathrm{KN} / \mathrm{m}$ Calender } & 11948 & 2.48 & 67.20 & 23.94 & 2.81 \\
\hline & 11949 & 2.49 & 64.65 & 24.31 & 2.66 \\
\hline & Average & 2.58 & 67.60 & 24.34 & 2.78 \\
\hline Case 2 & 11926 & 3.17 & 79.72 & 28.23 & 2.82 \\
\hline $246^{\circ} \mathrm{C}$ Roll Temp & 11930 & 3.03 & 77.56 & 26.78 & 2.90 \\
\hline \multirow[t]{3}{*}{ No Calender } & 11931 & 2.85 & 78.94 & 27.10 & 2.91 \\
\hline & 11932 & 3.35 & 79.01 & 27.47 & 2.88 \\
\hline & Average & 3.10 & 78.81 & 27.40 & 2.88 \\
\hline Case 6 & 11927 & 3.25 & 77.85 & 28.50 & 2.73 \\
\hline $246^{\circ} \mathrm{C}$ Roll Temp & 11928 & 3.02 & 79.48 & 28.27 & 2.81 \\
\hline $35 \mathrm{KN} / \mathrm{m}$ Calender & Average & 3.14 & 78.67 & 28.39 & 2.77 \\
\hline Case 4 & 11933 & 3.05 & 81.08 & 29.32 & 2.77 \\
\hline $260^{\circ} \mathrm{C}$ Roll Temp & 11934 & 2.89 & 77.44 & 26.78 & 2.89 \\
\hline \multirow[t]{3}{*}{ No Calender } & 11935 & 3.18 & 80.26 & 27.71 & 2.90 \\
\hline & 11936 & 2.91 & 78.06 & 27.15 & 2.88 \\
\hline & Average & 3.01 & 79.21 & 27.74 & 2.86 \\
\hline $204^{\circ} \mathrm{C}$ Roll Temp & 11950 & 2.72 & 72.20 & 26.50 & 2.72 \\
\hline $218^{\circ} \mathrm{C}$ Roll Temp & 11951 & 2.70 & 74.60 & 27.44 & 2.70 \\
\hline $232^{\circ} \mathrm{C}$ Roll Temp & 11952 & 2.90 & 69.18 & 26.41 & 2.62 \\
\hline $246^{\circ} \mathrm{C}$ Roll Temp & 11953 & 2.84 & 72.91 & 25.08 & 2.91 \\
\hline $260^{\circ} \mathrm{C}$ Roll Temp & 11954 & 3.14 & 74.38 & 26.93 & 2.76 \\
\hline $274^{\circ} \mathrm{C}$ Roll Temp & 11955 & 2.82 & 72.57 & 26.34 & 2.75 \\
\hline $288^{\circ} \mathrm{C}$ Roll Temp & 11956 & 2.80 & 77.23 & 25.78 & 3.00 \\
\hline WP - $43.8 \mathrm{KN} / \mathrm{m}$ Cal. & 11937 & 2.53 & 68.18 & 24.76 & 2.75 \\
\hline WP - $37.1 \mathrm{KN} / \mathrm{m}$ Cal. & 11938 & 2.86 & 70.82 & 24.34 & 2.91 \\
\hline WP - $10.5 \mathrm{KN} / \mathrm{m}$ Cal. & 11939 & 2.60 & 68.29 & 25.42 & 2.69 \\
\hline WP - $28.7 \mathrm{KN} / \mathrm{m}$ Cal. & 11940 & 2.34 & 69.08 & 25.11 & 2.75 \\
\hline WP - $19.8 \mathrm{kN} / \mathrm{m}$ Cal. & 11941 & 3.11 & 72.21 & 25.26 & 2.86 \\
\hline
\end{tabular}


Table E2. Average Physical Property Data.

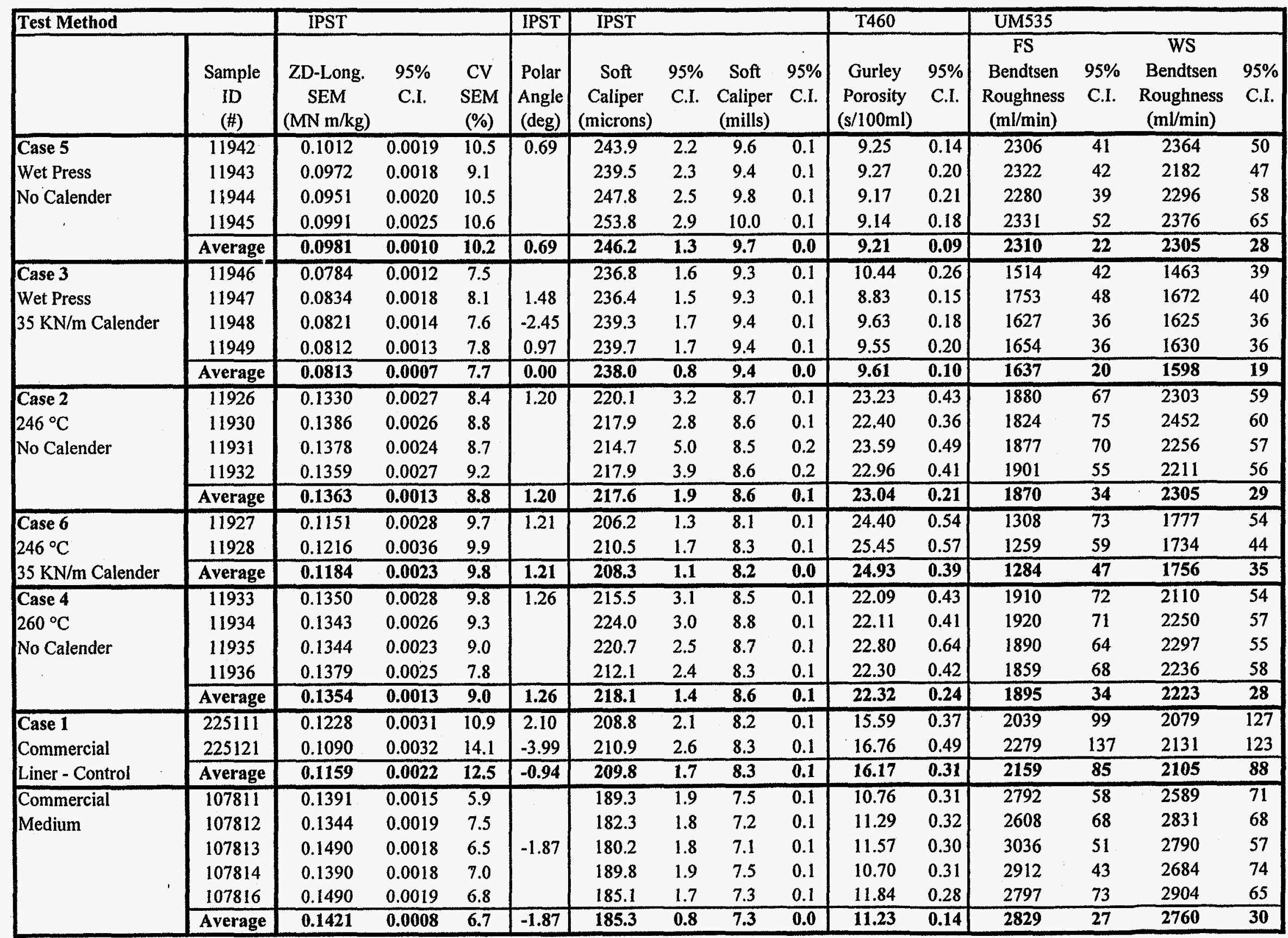


Table E2 cont. Average Physical Property Data.

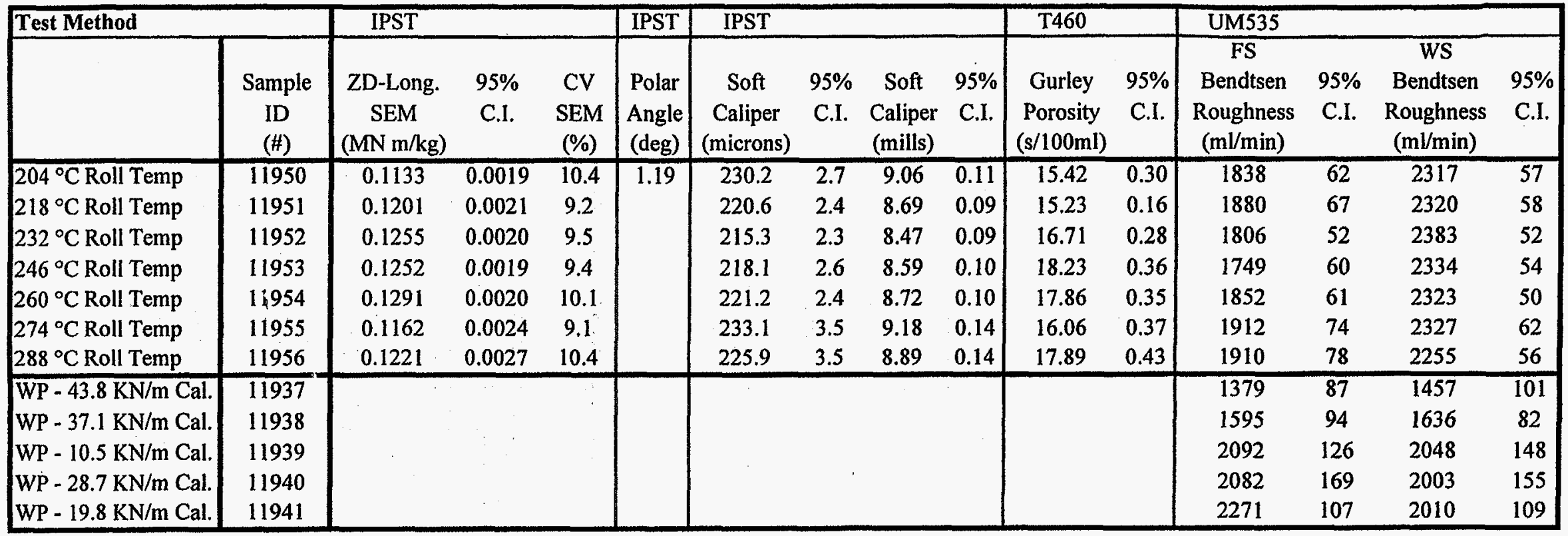


Table E2 cont. Average Physical Property Data.

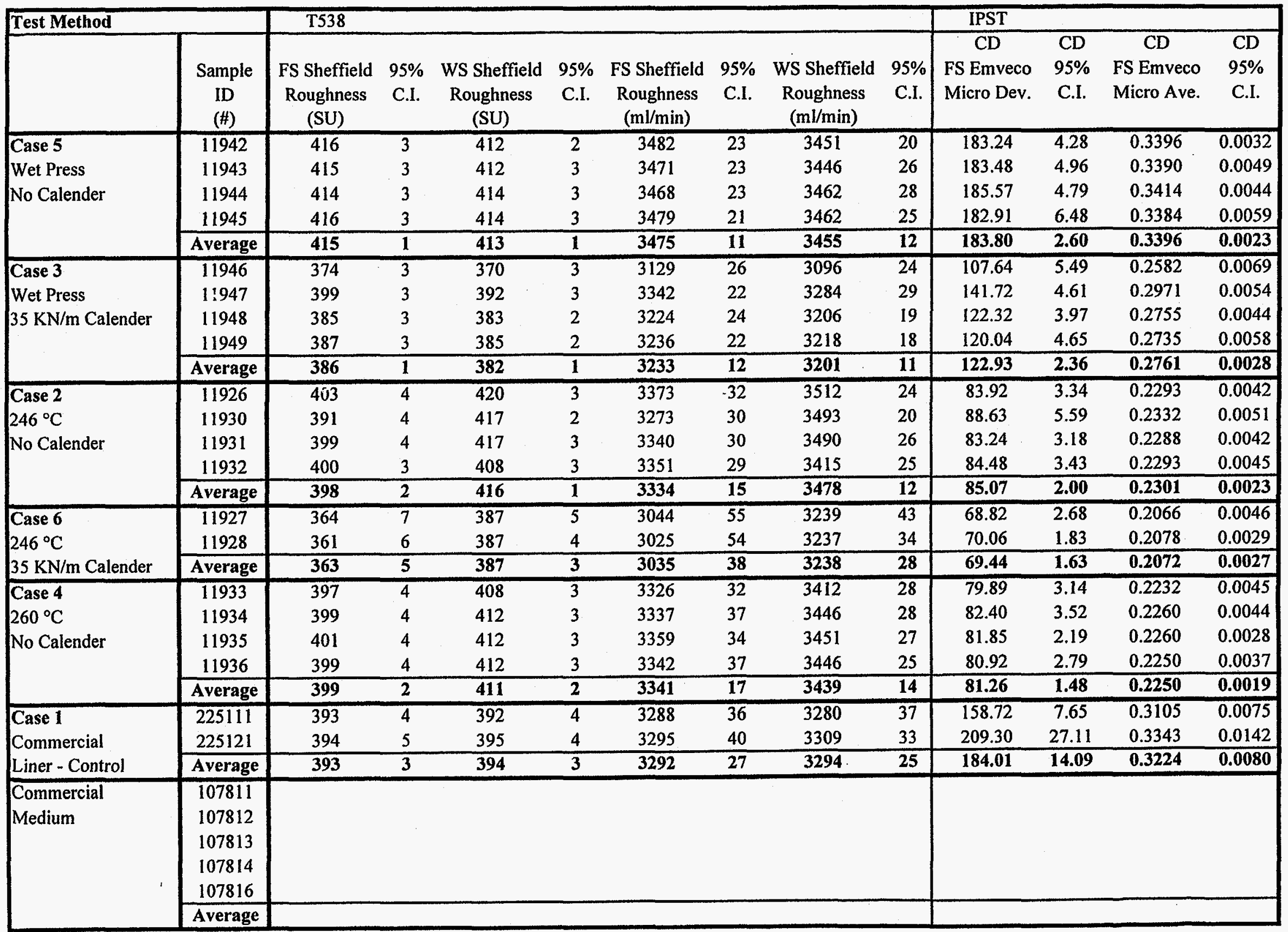


Table E2 cont. Average Physical Property Data.

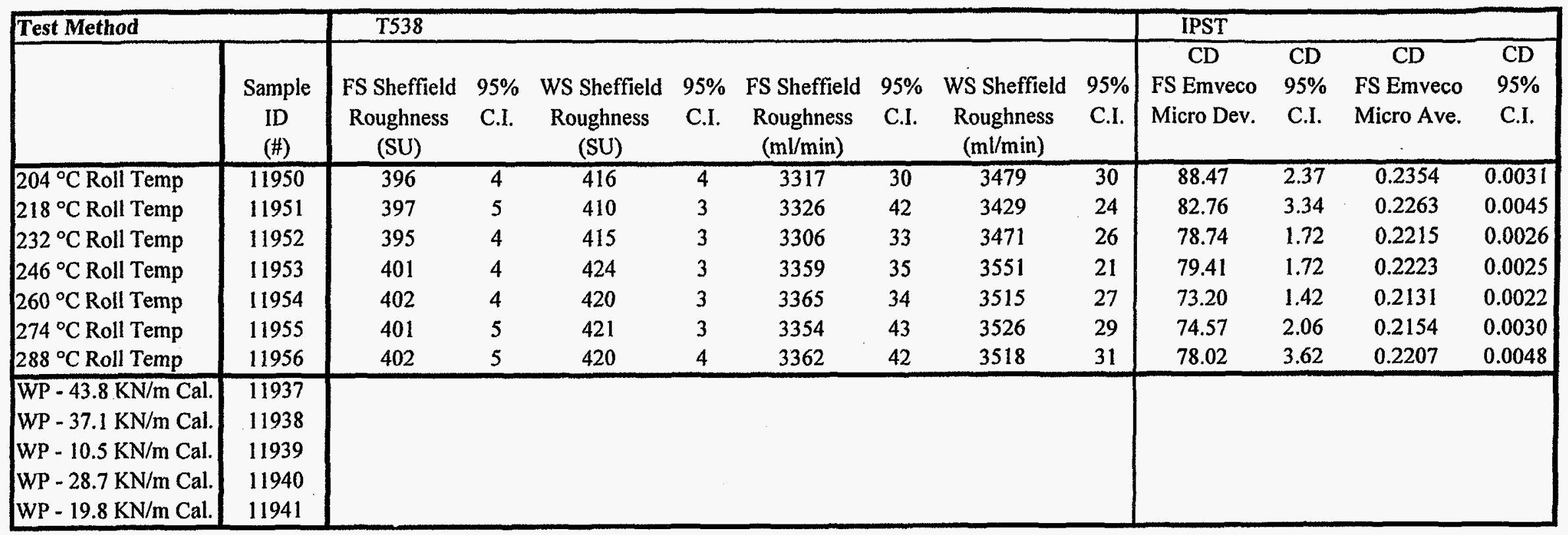


Table E2 cont. Average Physical Property Data.

\begin{tabular}{|c|c|c|c|c|c|c|c|c|c|c|c|c|c|}
\hline \multicolumn{2}{|l|}{ Test Method } & & & & & \multicolumn{8}{|c|}{ T527 (Diffuse) } \\
\hline & $\begin{array}{l}\text { Sample } \\
\text { ID } \\
(\#)\end{array}$ & $\begin{array}{c}\text { MD } \\
\text { FS Emveco } \\
\text { Micro Dev. }\end{array}$ & $\begin{array}{l}\text { MD } \\
95 \% \\
\text { C.I. }\end{array}$ & $\begin{array}{c}\text { MD } \\
\text { FS Emveco } \\
\text { Micro Ave. }\end{array}$ & $\begin{array}{c}\text { MD } \\
95 \% \\
\text { C.I. }\end{array}$ & $\begin{array}{l}\text { As Made } \\
\text { Color } \\
\text { Brightness }\end{array}$ & $\begin{array}{l}95 \% \\
\text { C.I. }\end{array}$ & $\begin{array}{c}L^{*} \\
\text { Lightness }\end{array}$ & $\begin{array}{l}95 \% \\
\text { C.I. }\end{array}$ & $\begin{array}{c}\mathrm{a}^{*} \\
\text { +Redness } \\
\text {-Greeness }\end{array}$ & $\begin{array}{l}95 \% \\
\text { C.I. }\end{array}$ & $\begin{array}{c}b^{*} \\
+ \text { Yellowness } \\
\text {-Blueness }\end{array}$ & $\begin{array}{l}95 \% \\
\text { C.I. }\end{array}$ \\
\hline Case 5 & 11942 & 142.22 & 8.50 & 0.2980 & 0.0073 & 15.59 & 0.08 & 59.35 & 0.16 & 5.63 & 0.60 & 24.14 & 0.09 \\
\hline Wet Press & 11943 & 137.69 & 5.64 & 0.2912 & 0.0062 & 15.68 & 0.05 & 59.39 & 0.18 & 5.80 & 0.12 & 24.19 & 0.04 \\
\hline \multirow[t]{3}{*}{ No Calender } & 11944 & 131.94 & 6.18 & 0.2860 & 0.0063 & 15.70 & 0.05 & 59.41 & 0.13 & 5.81 & 0.03 & 24.06 & 0.04 \\
\hline & 11945 & 138.97 & 8.27 & 0.2925 & 0.0076 & 15.67 & 0.07 & 59.37 & 0.20 & 5.79 & 0.12 & 24.02 & 0.29 \\
\hline & Average & 137.70 & 3.63 & 0.2919 & 0.0034 & 15.66 & 0.03 & 59.38 & 0.09 & 5.76 & 0.16 & 24.10 & 0.08 \\
\hline Case 3 & 11946 & 88.02 & 9.80 & 0.2306 & 0.0106 & 15.53 & 0.10 & 58.97 & 0.40 & 6.12 & 0.09 & 23.65 & 0.13 \\
\hline Wet Press & 11947 & 113.37 & 5.20 & 0.2660 & 0.0059 & 15.62 & 0.06 & 59.23 & 0.24 & 5.85 & 0.24 & 23.91 & 0.16 \\
\hline \multirow[t]{3}{*}{$35 \mathrm{KN} / \mathrm{m}$ Calender } & 11948 & 101.93 & 5.52 & 0.2504 & 0.0075 & 15.65 & 0.05 & 59.19 & 0.14 & 5.72 & 0.23 & 23.82 & 0.07 \\
\hline & 11949 & 99.83 & 5.52 & 0.2463 & 0.0076 & 15.59 & 0.09 & 59.08 & 0.28 & 5.77 & 0.25 & 23.74 & 0.14 \\
\hline & Average & 100.79 & 3.39 & 0.2483 & 0.0040 & 15.60 & 0.04 & 59.12 & 0.14 & 5.87 & 0.11 & 23.78 & 0.06 \\
\hline Case 2 & 11926 & 55.64 & 3.76 & 0.1847 & 0.0058 & 14.11 & 0.09 & 57.11 & 0.52 & 6.36 & 0.12 & 23.99 & 0.19 \\
\hline $246^{\circ} \mathrm{C}$ & 11930 & 54.96 & 2.70 & 0.1837 & 0.0042 & 14.11 & 0.09 & 57.07 & 0.54 & 6.40 & 0.03 & 23.86 & 0.06 \\
\hline \multirow[t]{3}{*}{ No Calender } & 11931 & 54.12 & 2.33 & 0.1831 & 0.0040 & 14.16 & 0.09 & 57.16 & 0.53 & 6.34 & 0.01 & 23.96 & 0.09 \\
\hline & 11932 & 54.88 & 2.74 & 0.1835 & 0.0057 & 14.06 & 0.06 & 57.04 & 0.36 & 6.37 & 0.15 & 23.95 & 0.05 \\
\hline & Average & 54.90 & 1.47 & 0.1837 & 0.0025 & 14.11 & 0.04 & 57.09 & 0.25 & 6.37 & 0.05 & 23.94 & 0.06 \\
\hline Case 6 & 11927 & 47.41 & 3.56 & 0.1689 & 0.0059 & 14.03 & 0.07 & 57.00 & 0.42 & 6.37 & 0.05 & 23.97 & 0.11 \\
\hline $246^{\circ} \mathrm{C}$ & 11928 & 47.98 & 2.18 & 0.1713 & 0.0040 & 14.10 & 0.05 & 57.09 & 0.40 & 6.37 & 0.20 & 23.95 & 0.13 \\
\hline $35 \mathrm{KN} / \mathrm{m}$ Calender & Average & 47.69 & 2.09 & 0.1701 & 0.0036 & 14.06 & 0.05 & 57.05 & 0.29 & 6.37 & 0.10 & 23.96 & 0.08 \\
\hline Case 4 & 11933 & 52.21 & 1.62 & 0.1771 & 0.0028 & 14.21 & 0.08 & 57.14 & 0.48 & 6.38 & 0.16 & 23.76 & 0.11 \\
\hline $260^{\circ} \mathrm{C}$ & 11934 & 52.32 & 3.25 & 0.1789 & 0.0052 & 14.25 & 0.10 & 57.20 & 0.60 & 6.29 & 0.17 & 23.75 & 0.22 \\
\hline \multirow[t]{3}{*}{ No Calender } & 11935 & 58.09 & 3.20 & 0.1873 & 0.0044 & 14.25 & 0.06 & 57.15 & 0.39 & 6.38 & 0.20 & 23.68 & 0.15 \\
\hline & 11936 & 50.53 & 3.91 & 0.1760 & 0.0067 & 14.18 & 0.08 & 57.03 & 0.45 & 6.37 & 0.22 & 23.65 & 0.05 \\
\hline & Average & 53.29 & 1.56 & 0.1798 & 0.0025 & 14.22 & 0.04 & 57.13 & 0.24 & 6.36 & 0.10 & 23.71 & 0.07 \\
\hline Case 1 & 225111 & 120.76 & 6.14 & 0.2678 & 0.0070 & 16.02 & 0.05 & 58.62 & 0.06 & 6.48 & 0.08 & 21.97 & 0.13 \\
\hline \multirow{2}{*}{$\begin{array}{l}\text { Commercial } \\
\text { Liner - Control }\end{array}$} & 225121 & 131.13 & 10.29 & 0.2793 & 0.0088 & 16.13 & 0.05 & 58.82 & 0.13 & 6.37 & 0.12 & 22.09 & 0.17 \\
\hline & Average & 125.94 & 5.99 & 0.2735 & 0.0056 & 16.08 & 0.03 & 58.72 & 0.07 & 6.42 & 0.07 & 22.03 & 0.11 \\
\hline \multirow{5}{*}{$\begin{array}{l}\text { Commercial } \\
\text { Medium }\end{array}$} & 107811 & & & & & & & & & & & & \\
\hline & 107812 & & & & & & & & & & & & \\
\hline & 107813 & & & & & & & & & & & & \\
\hline & $\begin{array}{l}107814 \\
107816\end{array}$ & & & & & & & & & & & & \\
\hline & Average & & & & & & & & & & & & \\
\hline
\end{tabular}


Table E2 cont. Average Physical Property Data.

\begin{tabular}{|c|c|c|c|c|c|c|c|c|c|c|c|c|c|}
\hline \multicolumn{2}{|l|}{ Test Method } & & & & & \multicolumn{8}{|c|}{ T527 (Diffuse) } \\
\hline & $\begin{array}{c}\text { Sample } \\
\text { ID } \\
\text { (\#) }\end{array}$ & $\begin{array}{c}\text { MD } \\
\text { FS Emveco } \\
\text { Micro Dev. }\end{array}$ & $\begin{array}{l}\text { MD } \\
95 \% \\
\text { C.I. }\end{array}$ & $\begin{array}{c}\text { MD } \\
\text { FS Emveco } \\
\text { Micro Ave. }\end{array}$ & $\begin{array}{l}\text { MD } \\
95 \% \\
\text { C.I. }\end{array}$ & $\begin{array}{l}\text { As Made } \\
\text { Color } \\
\text { Brightness }\end{array}$ & $\begin{array}{l}95 \% \\
\text { C.I. }\end{array}$ & $\begin{array}{c}\mathrm{L}^{*} \\
\text { Lightness }\end{array}$ & $\begin{array}{l}95 \% \\
\text { C.I. }\end{array}$ & $\begin{array}{c}\quad a^{*} \\
+ \text { +Redness } \\
\text {-Greeness }\end{array}$ & $\begin{array}{l}95 \% \\
\text { C.I. }\end{array}$ & $\begin{array}{c}\text { b* }^{*} \\
+ \text { Yellowness } \\
\text {-Blueness }\end{array}$ & $\begin{array}{l}95 \% \\
\text { C.I. }\end{array}$ \\
\hline $204^{\circ} \mathrm{C}$ Roll Temp & 11950 & 62.24 & 2.72 & 0.1953 & 0.0043 & 13.68 & 0.08 & 56.45 & 0.37 & 6.50 & 0.23 & 24.13 & 0.22 \\
\hline $218^{\circ} \mathrm{C}$ Roll Temp & 11951 & 56.85 & 4.03 & 0.1882 & 0.0074 & 13.57 & 0.08 & 56.25 & 0.51 & 6.54 & 0.24 & 23.98 & 0.15 \\
\hline $232^{\circ} \mathrm{C}$ Roll Temp & 11952 & 51.52 & 1.40 & 0.1788 & 0.0024 & 13.57 & 0.08 & 56.20 & 0.48 & 6.55 & 0.22 & 23.87 & 0.27 \\
\hline $246{ }^{\circ} \mathrm{C}$ Roll Temp & 11953 & 53.11 & 2.34 & 0.1800 & 0.0039 & 13.50 & 0.06 & 56.02 & 0.50 & 6.54 & 0.21 & 23.76 & 0.11 \\
\hline $260^{\circ} \mathrm{C}$ Roll Temp & 11954 & 47.28 & 1.32 & 0.1706 & 0.0028 & 13.81 & 0.14 & 56.45 & 0.83 & 6.35 & 0.23 & 23.72 & 0.31 \\
\hline $274^{\circ} \mathrm{C}$ Roll Temp & 11955 & 48.33 & 2.35 & 0.1709 & 0.0039 & 13.99 & 0.11 & 56.69 & 0.80 & 6.23 & 0.24 & 23.68 & 0.11 \\
\hline $288^{\circ} \mathrm{C}$ Roll Temp & 11956 & 45.78 & 2.75 & 0.1666 & 0.0053 & 14.15 & 0.08 & 56.83 & 0.44 & 6.09 & 0.05 & 23.54 & 0.10 \\
\hline $\begin{array}{l}\text { WP - } 43.8 \mathrm{KN} / \mathrm{m} \text { Cal. } \\
W P-37.1 \mathrm{KN} / \mathrm{m} \text { Cal. } \\
W P-10.5 \mathrm{KN} / \mathrm{m} \text { Cal. } \\
W P-28.7 \mathrm{KN} / \mathrm{m} \text { Cal. } \\
W P-19.8 \mathrm{KN} / \mathrm{m} \text { Cal. }\end{array}$ & $\begin{array}{l}11937 \\
11938 \\
11939 \\
11940 \\
11941\end{array}$ & & & & & & & & & & & & \\
\hline
\end{tabular}


Table E2 cont. Average Physical Property Data.

\begin{tabular}{|c|c|c|c|c|c|c|c|c|c|}
\hline \multicolumn{2}{|l|}{ Test Method } & \multicolumn{8}{|c|}{ T527 (Diffuse) } \\
\hline & \multirow[b]{2}{*}{$\begin{array}{l}\text { Sample } \\
\text { ID } \\
(\#)\end{array}$} & \multicolumn{8}{|c|}{ Conditioned and Exposed to Light 96 Hours } \\
\hline & & $\begin{array}{c}\text { Color } \\
\text { Brightness }\end{array}$ & $\begin{array}{c}95 \% \\
\text { C.I. }\end{array}$ & $\begin{array}{c}L^{*} \\
\text { Lightness }\end{array}$ & $\begin{array}{l}95 \% \\
\text { C.I. }\end{array}$ & $\begin{array}{c}\quad a^{*} \\
+ \text { Redness } \\
\text {-Greeness }\end{array}$ & $\begin{array}{c}95 \% \\
\text { C.I. }\end{array}$ & $\begin{array}{c}b^{*} \\
+ \text { Yellowness } \\
\text {-Blueness }\end{array}$ & $\begin{array}{l}95 \% \\
\text { C.I. }\end{array}$ \\
\hline Case 5 & 11942 & 14.92 & 0.06 & 57.83 & 0.24 & 7.07 & 0.10 & 21.78 & 0.09 \\
\hline Wet Press & 11943 & 14.93 & 0.05 & 57.79 & 0.14 & 7.17 & 0.15 & 21.70 & 0.15 \\
\hline \multirow[t]{3}{*}{ No Calender } & 11944 & 14.97 & 0.05 & 57.84 & 0.19 & 7.15 & 0.10 & 21.66 & 0.21 \\
\hline & 11945 & 14.97 & 0.04 & 57.84 & 0.19 & 7.18 & 0.15 & 21.67 & 0.07 \\
\hline & Average & 14.94 & 0.03 & 57.83 & 0.10 & 7.14 & 0.06 & 21.70 & 0.07 \\
\hline Case 3 & 11946 & 15.06 & 0.13 & 57.97 & 0.59 & 6.92 & 0.13 & 21.74 & 0.17 \\
\hline Wet Press & 11947 & 14.96 & 0.08 & 57.82 & 0.17 & 7.07 & 0.02 & 21.74 & 0.14 \\
\hline \multirow[t]{3}{*}{$35 \mathrm{KN} / \mathrm{m}$ Calender } & 11948 & 15.22 & 0.16 & 58.14 & 0.62 & 6.93 & 0.26 & 21.68 & 0.22 \\
\hline & 11949 & 15.23 & 0.13 & 58.17 & 0.62 & 6.88 & 0.26 & 21.78 & 0.35 \\
\hline & Average & 15.12 & 0.06 & 58.03 & 0.27 & 6.95 & 0.10 & 21.74 & 0.12 \\
\hline Case 2 & 11926 & 13.11 & 0.07 & 55.23 & 0.48 & 7.28 & 0.19 & 22.94 & 0.31 \\
\hline $246^{\circ} \mathrm{C}$ & 11930 & 13.15 & 0.08 & 55.16 & 0.30 & 7.18 & 0.08 & 22.78 & 0.09 \\
\hline \multirow[t]{3}{*}{ No Calender } & 11931 & 13.36 & 0.07 & 55.47 & 0.40 & 7.20 & 0.23 & 22.71 & 0.17 \\
\hline & 11932 & 13.38 & 0.04 & 55.46 & 0.09 & 7.19 & 0.19 & 22.67 & 0.18 \\
\hline & Average & 13.25 & 0.03 & 55.33 & 0.17 & 7.21 & 0.09 & 22.78 & 0.10 \\
\hline Case 6 & 11927 & 13.15 & 0.08 & 55.32 & 0.44 & 7.13 & 0.09 & 23.06 & 0.16 \\
\hline \multirow{2}{*}{$\begin{array}{l}246^{\circ} \mathrm{C} \\
35 \mathrm{KN} / \mathrm{m} \text { Calender }\end{array}$} & 11928 & 13.18 & 0.09 & 55.36 & 0.46 & 7.21 & 0.18 & 22.96 & 0.14 \\
\hline & Average & 13.16 & 0.06 & 55.34 & 0.32 & 7.17 & 0.10 & 23.01 & 0.11 \\
\hline Case 4 & 11933 & 13.48 & 0.06 & 55.54 & 0.26 & 7.10 & 0.25 & 22.52 & 0.17 \\
\hline $260^{\circ} \mathrm{C}$ & 11934 & 13.32 & 0.08 & 55.39 & 0.47 & 7.12 & 0.09 & 22.70 & 0.18 \\
\hline \multirow[t]{3}{*}{ No Calender } & 11935 & 13.58 & 0.10 & 55.67 & 0.53 & 7.15 & 0.34 & 22.48 & 0.20 \\
\hline & 11936 & 13.64 & 0.08 & 55.81 & 0.49 & 7.47 & 0.09 & 21.40 & 0.09 \\
\hline & Average & 13.50 & 0.04 & 55.60 & 0.22 & 7.21 & 0.11 & 22.28 & 0.08 \\
\hline \multirow{3}{*}{$\begin{array}{l}\text { Case } 1 \\
\text { Commercial } \\
\text { Liner - Control }\end{array}$} & 225111 & 15.93 & 0.03 & 58.78 & 0.12 & 6.52 & 0.06 & 21.14 & 0.09 \\
\hline & 225121 & 15.90 & 0.05 & 58.71 & 0.10 & 6.53 & 0.03 & 21.00 & 0.20 \\
\hline & Average & 15.91 & 0.03 & 58.74 & 0.08 & 6.53 & 0.04 & 21.07 & 0.11 \\
\hline \multirow{5}{*}{$\begin{array}{l}\text { Commercial } \\
\text { Medium }\end{array}$} & 107811 & & & & & & & & \\
\hline & 107812 & & & & & & & & \\
\hline & 107813 & & & & & & & & \\
\hline & $\begin{array}{l}107814 \\
107816\end{array}$ & & & & & & & & \\
\hline & Average & & & & & & & & \\
\hline
\end{tabular}


Table E2 cont. Average Physical Property Data.

\begin{tabular}{|c|c|c|c|c|c|c|c|c|c|}
\hline \multicolumn{2}{|l|}{ Test Method } & \multicolumn{8}{|c|}{ T527 (Diffuse) } \\
\hline & \multirow{3}{*}{$\begin{array}{c}\text { Sample } \\
\text { ID } \\
(\#)\end{array}$} & \multicolumn{8}{|c|}{ Conditioned and Exposed to Light 96 Hours } \\
\hline & & Color & $95 \%$ & $\mathrm{~L}^{*}$ & $95 \%$ & $a^{*}$ & $95 \%$ & $b^{*}$ & $95 \%$ \\
\hline & & Brightness & C.I. & Lightness & C.I. & $\begin{array}{l}\text { +Redness } \\
\text {-Greeness }\end{array}$ & C.I. & $\begin{array}{l}\text { +Yellowness } \\
\text {-Blueness }\end{array}$ & C.I. \\
\hline $204^{\circ} \mathrm{C}$ Roll Temp & 11950 & 13.04 & 0.05 & 55.10 & 0.22 & 7.53 & 0.22 & 21.71 & 0.14 \\
\hline $218^{\circ} \mathrm{C}$ Roll Temp & 11951 & 12.99 & 0.07 & 54.97 & 0.32 & 7.57 & 0.19 & 21.61 & 0.09 \\
\hline $232{ }^{\circ} \mathrm{C}$ Roll Temp & 11952 & 13.00 & 0.07 & 54.97 & 0.39 & 7.54 & 0.07 & 21.60 & 0.19 \\
\hline $246^{\circ} \mathrm{C}$ Roll Temp & 11953 & 12.90 & 0.08 & 54.68 & 0.43 & 7.51 & 0.11 & 21.30 & 0.14 \\
\hline $260^{\circ} \mathrm{C}$ Roll Temp & 11954 & 13.28 & 0.10 & 55.20 & 0.65 & 7.31 & 0.32 & 21.24 & 0.14 \\
\hline $274^{\circ} \mathrm{C}$ Roll Temp & 11955 & 13.40 & 0.08 & 55.35 & 0.33 & 7.26 & 0.09 & 21.24 & 0.11 \\
\hline $288^{\circ} \mathrm{C}$ Roll Temp & 11956 & 13.44 & 0.06 & 55.35 & 0.20 & 7.20 & 0.13 & 21.12 & 0.19 \\
\hline WP - $43.8 \mathrm{KN} / \mathrm{m} \mathrm{Cal}$. & 11937 & & & & & & & & \\
\hline $\mathrm{WP}-37.1 \mathrm{KN} / \mathrm{m}$ Cal. & 11938 & & & & & & & & \\
\hline $\mathrm{WP}-10.5 \mathrm{kN} / \mathrm{m}$ Cal. & 11939 & & & & & & & & \\
\hline $\mathrm{WP}-28.7 \mathrm{KN} / \mathrm{m} \mathrm{Cal}$. & 11940 & & & & & & & & \\
\hline WP - $19.8 \mathrm{KN} / \mathrm{m}$ Cal. & 11941 & & & & & & & & \\
\hline
\end{tabular}


Table E2 cont. Average Physical Property Data.

\begin{tabular}{|c|c|c|c|c|c|c|c|c|c|c|c|c|c|c|c|}
\hline \multirow[t]{4}{*}{ Test Method } & \multirow{4}{*}{$\begin{array}{l}\text { Sample } \\
\text { ID } \\
(\#)\end{array}$} & \multicolumn{8}{|c|}{ T524 (Directional) } & \multicolumn{2}{|l|}{ T480 } & \multicolumn{4}{|l|}{ T441 } \\
\hline & & \multicolumn{8}{|c|}{ Conditioned and Exposed to Light 96 Hours } & \multirow[b]{3}{*}{ Gloss } & \multirow{3}{*}{$\begin{array}{l}95 \% \\
\text { C.I. }\end{array}$} & \multirow{3}{*}{$\begin{array}{c}\text { FS Cobb } \\
\text { Sizing } \\
\left(\mathrm{g} / \mathrm{m}^{\wedge} 2\right)\end{array}$} & \multirow{3}{*}{$\begin{array}{l}95 \% \\
\text { C.I. }\end{array}$} & \multirow{3}{*}{$\begin{array}{l}\text { WS Cobb } \\
\text { Sizing } \\
\left(\mathrm{g} / \mathrm{m}^{\wedge} 2\right)\end{array}$} & \multirow{3}{*}{$\begin{array}{l}95 \% \\
\text { C.I. }\end{array}$} \\
\hline & & Color & $95 \%$ & $\mathrm{~L}^{*}$ & $95 \%$ & $a^{*}$ & $95 \%$ & $b^{*}$ & $95 \%$ & & & & & & \\
\hline & & Brightness & C.I. & Lightness & C.I. & $\begin{array}{l}\text { +Redness } \\
\text {-Greeness }\end{array}$ & C.I. & $\begin{array}{l}\text { +Yellowness } \\
\text {-Blueness }\end{array}$ & C.I. & & & & & & \\
\hline Case 5 & 11942 & 15.20 & 0.18 & 58.47 & 0.69 & $\overline{6.66}$ & 0.33 & 22.33 & 0.19 & 7.43 & 0.24 & 372.9 & 4.3 & 366.3 & 8.6 \\
\hline Wet Press & 11943 & 15.23 & 0.13 & 58.48 & 0.22 & 6.77 & 0.04 & 22.28 & 0.19 & 7.15 & 0.08 & 326.1 & 5.4 & 320.8 & 6.4 \\
\hline \multirow{3}{*}{ No Calender } & 11944 & 15.27 & 0.11 & 58.51 & 0.44 & 6.80 & 0.21 & 22.24 & 0.13 & 7.19 & 0.11 & 337.0 & 5.6 & 335.7 & 5.0 \\
\hline & 11945 & 15.12 & 0.17 & 58.32 & 0.43 & 6.80 & 0.08 & 22.27 & 0.29 & 7.20 & 0.07 & 336.2 & 7.4 & 328.7 & 8.2 \\
\hline & Average & 15.20 & 0.08 & 58.45 & 0.24 & 6.76 & 0.10 & 22.28 & 0.10 & 7.24 & 0.07 & 343.1 & 2.9 & 337.9 & 3.6 \\
\hline Case 3 & 11946 & 15.76 & 0.24 & 59.17 & 0.80 & 6.68 & 0.09 & 22.35 & 0.12 & 9.09 & 0.13 & 317.3 & 6.0 & 315.6 & 4.1 \\
\hline Wet Press & $11947^{\circ}$ & 15.33 & 0.15 & 58.69 & 0.24 & 6.81 & 0.25 & 22.43 & 0.07 & 7.97 & 0.28 & 359.7 & 8.2 & 364.9 & 6.8 \\
\hline \multirow[t]{3}{*}{$35 \mathrm{KN} / \mathrm{m}$ Calender } & 11948 & 15.92 & 0.19 & 59.35 & 0.35 & 6.61 & 0.17 & 22.21 & 0.19 & 8.90 & 0.18 & 314.7 & 4.0 & 313.0 & 6.4 \\
\hline & 11949 & 16.03 & 0.18 & 59.55 & 0.60 & 6.54 & 0.13 & 22.29 & 0.11 & 9.06 & 0.12 & 312.8 & 5.0 & 303.2 & 8.8 \\
\hline & Average & 15.76 & 0.10 & 59.19 & 0.27 & 6.66 & 0.09 & 22.32 & 0.07 & 8.76 & 0.09 & 326.1 & 3.0 & 324.2 & 3.4 \\
\hline Case 2 & 11926 & 13.47 & $\overline{0.13}$ & 55.97 & 0.54 & 7.40 & 0.18 & 22.14 & 0.01 & 12.71 & 0.17 & 304.7 & 9.4 & 297.2 & 7.4 \\
\hline $246^{\circ} \mathrm{C}$ & 11930 & 13.47 & 0.14 & 55.82 & 0.26 & 7.33 & 0.22 & 21.94 & 0.13 & 12.02 & 0.17 & 301.6 & 6.2 & 301.8 & 5.5 \\
\hline \multirow[t]{3}{*}{ No Calender } & 11931 & 13.36 & 0.13 & 55.73 & 0.40 & 7.37 & 0.10 & 22.05 & 0.07 & 12.03 & 0.17 & 285.4 & 7.8 & 290.4 & 8.4 \\
\hline & 11932 & 13.62 & 0.13 & 56.02 & 0.45 & 7.26 & 0.11 & 21.91 & 0.11 & 12.13 & 0.16 & 288.4 & 9.3 & 299.0 & 11.8 \\
\hline & Average & 13.48 & 0.07 & 55.88 & 0.21 & 7.34 & 0.08 & 22.01 & 0.05 & 12.22 & 0.08 & 295.0 & 4.1 & 297.1 & 4.3 \\
\hline Case 6 & 11927 & 13.55 & 0.17 & 56.05 & 0.40 & 7.36 & 0.15 & 22.17 & 0.21 & 13.15 & 0.27 & 293.8 & 2.7 & 295.0 & 4.8 \\
\hline $246^{\circ} \mathrm{C}$ & 11928 & 13.41 & 0.14 & 55.89 & 0.62 & 7.35 & 0.14 & 22.21 & 0.13 & 12.93 & 0.18 & 291.7 & 6.0 & 293.9 & 6.8 \\
\hline $35 \mathrm{KN} / \mathrm{m}$ Calender & Average & 13.48 & 0.11 & 55.97 & 0.37 & 7.36 & 0.10 & 22.19 & 0.12 & 13.04 & 0.16 & 292.8 & 3.3 & 294.5 & 4.2 \\
\hline Case 4 & 11933 & 13.68 & 0.15 & 56.05 & 0.34 & 7.23 & 0.17 & 21.80 & 0.16 & 13.63 & 0.22 & 300.3 & 4.8 & 294.8 & 6.0 \\
\hline $260^{\circ} \mathrm{C}$ & 11934 & 13.70 & 0.17 & 56.12 & 0.48 & 7.24 & 0.08 & 21.84 & 0.20 & 13.38 & 0.37 & 308.2 & 5.8 & 309.4 & 8.8 \\
\hline \multirow[t]{3}{*}{ No Calender } & 11935 & 13.76 & 0.17 & 56.18 & 0.53 & 7.18 & 0.13 & 21.83 & 0.19 & 13.24 & 0.23 & 301.0 & 6.3 & 295.4 & 5.5 \\
\hline & 11936 & 13.78 & 0.17 & 56.34 & 0.92 & 7.10 & 0.20 & 22.03 & 0.08 & 13.48 & 0.16 & 289.0 & 8.7 & 270.7 & 6.4 \\
\hline & Average & 13.73 & 0.08 & $\overline{56.17}$ & 0.30 & 7.19 & 0.08 & 21.88 & 0.08 & 13.43 & 0.13 & 299.6 & 3.3 & 292.6 & 3.4 \\
\hline Case 1 & 225111 & 15.93 & 0.03 & 58.78 & 0.12 & 6.52 & 0.06 & 21.14 & 0.09 & 16.03 & 0.18 & 38.8 & 2.1 & 55.4 & 4.2 \\
\hline \multirow{2}{*}{$\begin{array}{l}\text { Commercial } \\
\text { Liner - Control }\end{array}$} & 225121 & 15.90 & 0.05 & 58.71 & 0.10 & 6.53 & 0.03 & 21.00 & 0.20 & 15.87 & 0.21 & 43.4 & 3.5 & 63.9 & 3.8 \\
\hline & Average & 15.91 & 0.03 & 58.74 & 0.08 & 6.53 & 0.04 & 21.07 & 0.11 & 15.95 & 0.14 & 41.1 & 2.1 & 59.7 & 2.8 \\
\hline \multirow{6}{*}{$\begin{array}{l}\text { Commercial } \\
\text { Medium }\end{array}$} & 107811 & & & & & & & & & & & 208.8 & 5.1 & 211.6 & 4.9 \\
\hline & 107812 & & & & & & & & & & & 216.0 & 4.6 & 219.3 & 3.3 \\
\hline & 107813 & & & & & & & & & & & 203.1 & 5.9 & 203.7 & 8.4 \\
\hline & 107814 & & & & & & & & & & & 224.2 & 7.4 & 243.4 & 13.8 \\
\hline & 107816 & & & & & & & & & & & 255.8 & 12.3 & 233.1 & 11.0 \\
\hline & Average & & & & & & & & & & & 221.6 & 3.4 & 222.2 & 4.1 \\
\hline
\end{tabular}


Table E2 cont. Average Physical Property Data.

\begin{tabular}{|c|c|c|c|c|c|c|c|c|c|c|c|c|c|c|c|}
\hline \multicolumn{2}{|l|}{ Test Method } & \multicolumn{8}{|c|}{ T524 (Directional) } & \multicolumn{2}{|l|}{$T 480$} & \multicolumn{4}{|l|}{ T441 } \\
\hline & & Conditioned & and $E_{x}$ & posed to Lig & ht 96I & Hours & & & & & & & & & \\
\hline & Sample & Color & $95 \%$ & $\mathrm{~L}^{*}$ & $95 \%$ & $a^{*}$ & $95 \%$ & $b^{*}$ & $95 \%$ & & $95 \%$ & FS Cobb & $95 \%$ & WS Cobb & $95 \%$ \\
\hline & $\begin{array}{l}\text { ID } \\
(\#)\end{array}$ & Brightness & C.I. & Lightness & C.I. & $\begin{array}{l}\text { +Redness } \\
\text {-Greeness }\end{array}$ & C.I. & $\begin{array}{l}\text { +Yellowness } \\
\text {-Blueness }\end{array}$ & C.I. & Gloss & C.I. & $\begin{array}{c}\text { Sizing } \\
\left(\mathrm{g} / \mathrm{m}^{\wedge} 2\right)\end{array}$ & C.I. & $\begin{array}{c}\text { Sizing } \\
\left(\mathrm{g} / \mathrm{m}^{\wedge} 2\right)\end{array}$ & C.I. \\
\hline $204^{\circ} \mathrm{C}$ Roll Temp & 11950 & 13.07 & 0.18 & 55.44 & 0.72 & $\overline{7.34}$ & 0.29 & 22.30 & 0.27 & 10.89 & 0.13 & 330.2 & 8.0 & 332.5 & 5.0 \\
\hline $218^{\circ} \mathrm{C}$ Roll Temp & 11951 & 13.17 & 0.11 & 55.50 & 0.48 & 7.28 & 0.26 & 22.15 & 0.20 & 11.69 & 0.19 & 316.5 & 5.7 & 319.4 & 8.6 \\
\hline $232^{\circ} \mathrm{C}$ Roll Temp & 11952 & 13.01 & 0.14 & 55.27 & 0.46 & 7.36 & 0.24 & 22.13 & 0.22 & 12.48 & 0.18 & 306.6 & 6.7 & 309.7 & 7.2 \\
\hline $246^{\circ} \mathrm{C}$ Roll Temp & 11953 & 13.08 & 0.16 & 55.25 & 0.41 & 7.26 & 0.17 & 21.95 & 0.06 & 13.53 & 0.20 & 311.4 & 7.4 & 305.2 & 8.6 \\
\hline $260^{\circ} \mathrm{C}$ Roll Temp & 11954 & 13.29 & 0.19 & 55.59 & 0.90 & 7.09 & 0.26 & 21.98 & 0.11 & 14.97 & 0.23 & 305.5 & 6.8 & 311.8 & 8.5 \\
\hline $274^{\circ} \mathrm{C}$ Roll Temp & 11955 & 13.42 & 0.15 & 55.76 & 0.14 & 7.11 & 0.08 & 21.97 & 0.10 & 15.85 & 0.26 & 310.9 & 6.8 & 315.6 & 6.9 \\
\hline $288^{\circ} \mathrm{C}$ Roll Temp & 11956 & 13.36 & 0.17 & 55.65 & 0.42 & 7.06 & 0.10 & 21.91 & 0.13 & 17.46 & 0.19 & 293.6 & 6.7 & 300.2 & 5.1 \\
\hline $\mathrm{WP}$ - $43.8 \mathrm{KN} / \mathrm{m}$ Cal. & 11937 & & & & & & & & & & & & & & \\
\hline WP - $37.1 \mathrm{kN} / \mathrm{m}$ Cal. & 11938 & & & & & & & & & & & & & & \\
\hline WP - $10.5 \mathrm{KN} / \mathrm{m}$ Cal. & 11939 & & & & & & & & & & & & & & \\
\hline $\mathrm{WP}-28.7 \mathrm{KN} / \mathrm{m}$ Cal. & 11940 & & & & & & & & & & & & & & \\
\hline WP $-19.8 \mathrm{KN} / \mathrm{m}$ Cal. & 11941 & & & & & & & & & & & & & & \\
\hline
\end{tabular}


Table E2 cont. Average Physical Property Data.

\begin{tabular}{|c|c|c|c|c|c|c|c|c|c|c|c|c|c|}
\hline \multicolumn{2}{|l|}{ Test Method } & \multicolumn{4}{|l|}{$T 403$} & \multicolumn{4}{|l|}{ IPST } & \multicolumn{4}{|l|}{ T826 } \\
\hline & $\begin{array}{l}\text { Sample } \\
\text { ID } \\
(\#)\end{array}$ & $\begin{array}{c}\text { Mullen } \\
\text { Burst } \\
\text { (psi) }\end{array}$ & $\begin{array}{l}95 \% \\
\text { C.I. }\end{array}$ & $\begin{array}{c}\text { Mullen } \\
\text { Burst } \\
(\mathrm{kPa})\end{array}$ & $\begin{array}{l}95 \% \\
\text { C.I. }\end{array}$ & $\begin{array}{c}\text { MD Crack } \\
\text { Angle } \\
\text { (deg) }\end{array}$ & $\begin{array}{l}95 \% \\
\text { C.I. }\end{array}$ & $\begin{array}{c}\text { CD Crack } \\
\text { Angle } \\
\text { (deg) }\end{array}$ & $\begin{array}{l}95 \% \\
\text { C.I. }\end{array}$ & $\begin{array}{c}\text { CD } \\
\text { STFI } \\
\text { (lbf/in) }\end{array}$ & $\begin{array}{l}95 \% \\
\text { C.I. }\end{array}$ & $\begin{array}{c}\text { MD } \\
\text { STFI } \\
\text { (lbf/in) }\end{array}$ & $\begin{array}{l}95 \% \\
\text { C.I. }\end{array}$ \\
\hline Case 5 & 11942 & 67.2 & 2.6 & 463.4 & $\overline{18.3}$ & 64.4 & 4.2 & 77.3 & 5.3 & 14.89 & 0.38 & 27.08 & 0.54 \\
\hline Wet Press & 11943 & 66.3 & 2.1 & 457.1 & 14.8 & 63.1 & 2.8 & 66.4 & 5.4 & 15.34 & 0.38 & 26.73 & 0.48 \\
\hline \multirow[t]{3}{*}{ No Calender } & 11944 & 67.2 & 2.6 & 463.6 & 17.7 & 66.0 & 3.5 & 71.0 & 6.3 & 15.79 & 0.38 & 27.11 & 0.48 \\
\hline & 11945 & 66.2 & 3.0 & 456.2 & 20.4 & 66.7 & 2.2 & 47.5 & 7.9 & 15.16 & 0.42 & 26.64 & 0.45 \\
\hline & Average & 66.7 & 1.3 & 460.1 & 8.9 & 65.1 & 1.6 & 65.6 & 3.2 & 15.30 & 0.20 & 26.89 & 0.24 \\
\hline Case 3 & 11946 & 65.7 & 2.0 & 452.8 & 14.0 & 73.8 & 3.1 & 74.1 & 3.9 & 15.37 & 0.34 & 27.12 & 0.51 \\
\hline Wet Press & 11947 & 64.3 & 2.3 & 443.0 & 15.9 & 64.5 & 4.6 & 70.2 & 4.7 & 14.73 & 0.29 & 26.31 & 0.48 \\
\hline \multirow{3}{*}{$35 \mathrm{KN} / \mathrm{m}$ Calender } & 11948 & 63.7 & 2.2 & 439.5 & 15.4 & 68.8 & 2.3 & 71.0 & 5.6 & 15.22 & 0.36 & 26.89 & 0.39 \\
\hline & 11949 & 64.4 & 2.6 & 444.3 & 18.2 & 69.0 & 3.2 & 68.3 & 5.2 & 14.82 & 0.32 & 25.92 & 0.44 \\
\hline & Average & 64.5 & 1.2 & 444.9 & 8.0 & 69.0 & 1.7 & 70.9 & 2.4 & 15.03 & 0.16 & 26.56 & $\overline{0.23}$ \\
\hline Case 2 & 11926 & 78.6 & 2.4 & 541.9 & 16.3 & 65.3 & 3.7 & 71.2 & 5.6 & 16.60 & 0.39 & 27.61 & 0.58 \\
\hline $246^{\circ} \mathrm{C}$ & 11930 & 77.9 & 2.1 & 537.1 & 14.6 & 69.8 & 2.5 & 72.1 & 3.5 & 16.66 & 0.37 & 29.83 & 0.53 \\
\hline \multirow[t]{3}{*}{ No Calender } & 11931 & 78.6 & 2.6 & 542.0 & 17.8 & 64.6 & 4.5 & 66.1 & 2.6 & 16.66 & 0.38 & 29.71 & 0.49 \\
\hline & 11932 & 77.5 & 2.0 & 534.2 & 13.6 & 71.9 & 2.7 & 69.7 & 4.2 & 16.31 & 0.36 & 28.58 & 0.54 \\
\hline & Average & 78.1 & 1.1 & 538.8 & 7.8 & 67.9 & 1.7 & 69.8 & 2.1 & 16.56 & 0.19 & 28.93 & 0.27 \\
\hline Case 6 & 11927 & 76.7 & 2.8 & 529.0 & 19.2 & 64.6 & 2.9 & 65.8 & 4.0 & 16.36 & 0.34 & 28.45 & 0.50 \\
\hline $246^{\circ} \mathrm{C}$ & 11928 & 77.9 & 2.7 & 537.3 & 18.5 & 68.2 & 2.9 & 65.3 & 4.4 & 16.49 & 0.41 & 29.34 & 0.52 \\
\hline $35 \mathrm{KN} / \mathrm{m}$ Calender & Average & 77.3 & 1.9 & 533.2 & 13.4 & 66.4 & 2.1 & 65.6 & 3.0 & 16.42 & 0.27 & 28.89 & 0.36 \\
\hline Case 4 & 11933 & 73.6 & 2.5 & 507.3 & 17.1 & 58.0 & 2.5 & 70.3 & 3.4 & 16.27 & 0.42 & 28.10 & $\overline{0.49}$ \\
\hline $260^{\circ} \mathrm{C}$ & 11934 & 75.2 & 2.4 & 518.7 & 16.9 & 69.3 & 2.9 & 69.3 & 4.0 & 16.62 & 0.36 & 29.19 & 0.53 \\
\hline \multirow[t]{3}{*}{ No Calender } & 11935 & 78.0 & 2.6 & 537.9 & 18.2 & .71 .3 & 2.4 & 70.4 & 3.6 & 16.56 & 0.28 & 29.67 & 0.51 \\
\hline & 11936 & 75.3 & 2.7 & 519.3 & 18.9 & 69.8 & 3.0 & 69.1 & 4.5 & 16.74 & 0.42 & 30.16 & 0.47 \\
\hline & Average & .75 .5 & 1.3 & 520.8 & 8.9 & 67.1 & 1.4 & 69.8 & 1.9 & 16.55 & 0.19 & 29.28 & 0.25 \\
\hline Case 1 & 225111 & 85.0 & 3.6 & 586.4 & 24.7 & 70.2 & 2.8 & 84.2 & 3.4 & 17.88 & 0.38 & 32.88 & $\overline{0.65}$ \\
\hline \multirow{2}{*}{$\begin{array}{l}\text { Commercial } \\
\text { Liner - Control }\end{array}$} & 225121 & 81.3 & 2.7 & 560.6 & 18.3 & 72.8 & 2.9 & 83.9 & 4.4 & 18.12 & 0.42 & 33.19 & 0.58 \\
\hline & Average & 83.2 & 2.2 & 573.5 & 15.4 & 71.5 & 2.0 & 84.1 & 2.8 & 18.00 & 0.28 & 33.04 & 0.43 \\
\hline Commercial & 107811 & $\overline{35.4}$ & 0.8 & 244.2 & 5.8 & & & & & 11.06 & 0.24 & 21.31 & 0.55 \\
\hline \multirow[t]{5}{*}{ Medium } & 107812 & 34.7 & 1.0 & 239.1 & 7.0 & & & & & 10.95 & 0.24 & 20.02 & 0.30 \\
\hline & 107813 & 35.3 & 1.3 & 243.4 & 8.7 & & & & & 10.72 & 0.21 & 20.47 & 0.32 \\
\hline & 107814 & 35.1 & 1.1 & 242.3 & 7.7 & & & & & 11.02 & 0.17 & 20.82 & 0.46 \\
\hline & 107816 & 35.4 & 1.2 & 243.9 & 8.3 & & & & & 10.91 & 0.17 & 21.18 & 0.36 \\
\hline & Average & 35.2 & 0.5 & 242.6 & 3.4 & & & & & 10.93 & 0.09 & 20.76 & 0.18 \\
\hline
\end{tabular}


Table E2 cont. Average Physical Property Data.

\begin{tabular}{|c|c|c|c|c|c|c|c|c|c|c|c|c|c|}
\hline \multicolumn{2}{|l|}{ Test Method } & \multicolumn{4}{|l|}{$\mathrm{T} 403$} & \multicolumn{4}{|l|}{ IPST } & \multicolumn{4}{|l|}{ T826 } \\
\hline & $\begin{array}{c}\text { Sample } \\
\text { ID } \\
(\#)\end{array}$ & $\begin{array}{c}\text { Mullen } \\
\text { Burst } \\
\text { (psi) }\end{array}$ & $\begin{array}{l}95 \% \\
\text { C.I. }\end{array}$ & $\begin{array}{c}\text { Mullen } \\
\text { Burst } \\
(\mathrm{kPa})\end{array}$ & $\begin{array}{l}95 \% \\
\text { C.I. }\end{array}$ & $\begin{array}{c}\text { MD Crack } \\
\text { Angle } \\
\text { (deg) }\end{array}$ & $\begin{array}{c}95 \% \\
\text { C.I. }\end{array}$ & $\begin{array}{c}\text { CD Crack } \\
\text { Angle } \\
\text { (deg) }\end{array}$ & $\begin{array}{c}95 \% \\
\text { C.I. }\end{array}$ & $\begin{array}{c}\text { CD } \\
\text { STFI } \\
\text { (lbf/in) }\end{array}$ & $\begin{array}{c}95 \% \\
\text { C.I. }\end{array}$ & $\begin{array}{c}\text { MD } \\
\text { STFI } \\
\text { (lbf/in) }\end{array}$ & $\begin{array}{c}95 \% \\
\text { C.I. }\end{array}$ \\
\hline $204^{\circ} \mathrm{C}$ Roll Temp & 11950 & 68.3 & 2.4 & 470.9 & 16.7 & 74.1 & 3.9 & 81.1 & 4.3 & 15.83 & 0.28 & 27.62 & 0.38 \\
\hline $218^{\circ} \mathrm{C}$ Roll Temp & 11951 & 69.2 & 2.3 & 477.2 & 15.6 & 68.5 & 3.7 & 67.1 & 5.8 & 16.07 & 0.32 & 28.41 & 0.36 \\
\hline $232^{\circ} \mathrm{C}$ Roll Temp & 11952 & 67.8 & 2.5 & 467.3 & 17.2 & 71.1 & 5.5 & 67.4 & 7.6 & 15.54 & 0.26 & 27.51 & 0.38 \\
\hline $246^{\circ} \mathrm{C}$ Roll Temp & 11953 & 69.8 & 2.7 & 481.1 & 18.8 & 79.6 & 3.0 & 83.7 & 4.4 & 15.76 & 0.30 & 27.55 & 0.37 \\
\hline $260^{\circ} \mathrm{C}$ Roll Temp & 11954 & 70.7 & 2.4 & 487.7 & 16.7 & 72.4 & 2.1 & 75.4 & 3.3 & 16.34 & 0.30 & 28.27 & 0.34 \\
\hline $274^{\circ} \mathrm{C}$ Roll Temp & 11955 & 71.8 & 2.5 & 495.2 & 17.2 & 74.0 & 3.9 & 72.5 & 3.7 & 16.33 & 0.35 & 28.27 & 0.49 \\
\hline $288^{\circ} \mathrm{C}$ Roll Temp & 11956 & 72.2 & 2.3 & 497.7 & 15.8 & 77.3 & 3.7 & 80.5 & 3.9 & 15.98 & 0.32 & 28.74 & 0.45 \\
\hline $\begin{array}{l}\text { WP - } 43.8 \mathrm{KN} / \mathrm{m} \text { Cal. } \\
W P-37.1 \mathrm{KN} / \mathrm{m} \text { Cal. } \\
W P-10.5 \mathrm{KN} / \mathrm{m} \text { Cal. } \\
W P-28.7 \mathrm{KN} / \mathrm{m} \mathrm{Cal} . \\
W P-19.8 \mathrm{KN} / \mathrm{m} \mathrm{Cal} .\end{array}$ & $\begin{array}{l}11937 \\
11938 \\
11939 \\
11940 \\
11941\end{array}$ & & & & & & & & & & & & \\
\hline
\end{tabular}


Table E2 cont. Average Physical Property Data.

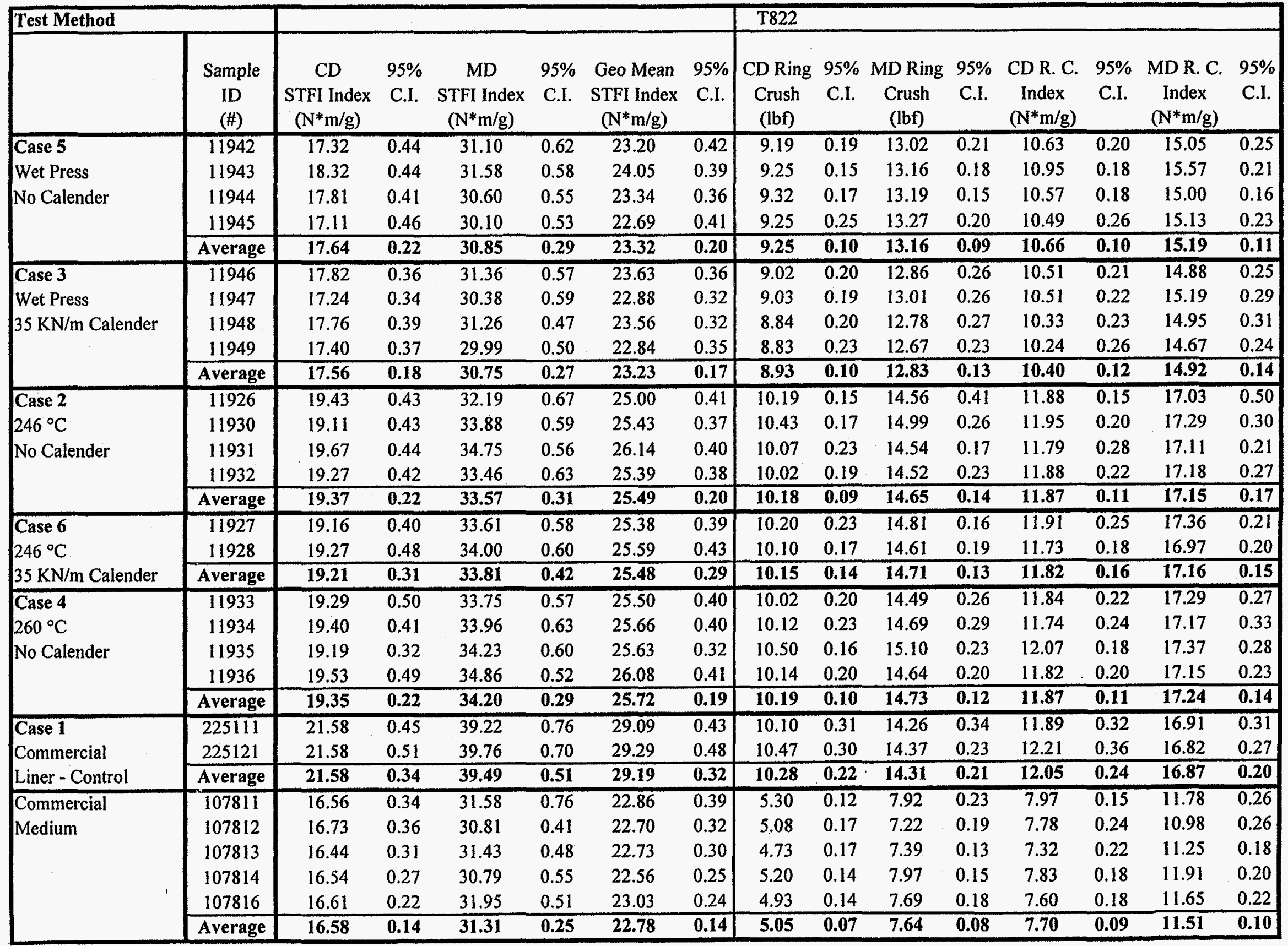


Table E2 cont. Average Physical Property Data.

\begin{tabular}{|c|c|c|c|c|c|c|c|c|c|c|c|c|c|c|c|}
\hline \multicolumn{2}{|l|}{ Test Method } & & & & & & & \multicolumn{8}{|l|}{ T822 } \\
\hline & $\begin{array}{l}\text { Sample } \\
\text { ID } \\
(\#)\end{array}$ & $\begin{array}{c}\text { CD } \\
\text { STFI Index } \\
\left(\mathrm{N}^{*} \mathrm{~m} / \mathrm{g}\right)\end{array}$ & $\begin{array}{c}95 \% \\
\text { C.I. }\end{array}$ & $\begin{array}{c}\text { MD } \\
\text { STFI Index } \\
\left(\mathrm{N}^{*} \mathrm{~m} / \mathrm{g}\right)\end{array}$ & $\begin{array}{c}95 \% \\
\text { C.I. }\end{array}$ & $\begin{array}{c}\text { Geo Mean } \\
\text { STFI Index } \\
\left(\mathrm{N}^{*} \mathrm{~m} / \mathrm{g}\right)\end{array}$ & $\begin{array}{c}95 \% \\
\text { C.I. }\end{array}$ & $\begin{array}{c}\text { CD Ring } \\
\text { Crush } \\
\text { (lbf) }\end{array}$ & $\begin{array}{l}95 \% \\
\text { C.I. }\end{array}$ & $\begin{array}{l}\text { MD Ring } \\
\text { Crush } \\
\text { (lbf) }\end{array}$ & $\begin{array}{c}95 \% \\
\text { C.I. }\end{array}$ & $\begin{array}{c}\text { CD R. C. } \\
\text { Index } \\
\left(N^{*} \mathrm{~m} / \mathrm{g}\right)\end{array}$ & $\begin{array}{l}95 \% \\
\text { C.I. }\end{array}$ & $\begin{array}{c}\text { MD R. C. } \\
\text { Index } \\
\left(N^{*} \mathrm{~m} / \mathrm{g}\right)\end{array}$ & $\begin{array}{c}95 \% \\
\text { C.I. }\end{array}$ \\
\hline $204^{\circ} \mathrm{C}$ Roll Temp & 11950 & 18.55 & 0.33 & 32.26 & 0.46 & 24.46 & 0.32 & 9.47 & 0.16 & 13.17 & 0.29 & 11.09 & 0.18 & 15.38 & $\overline{0.35}$ \\
\hline $218^{\circ} \mathrm{C}$ Roll Temp & 11951 & 18.76 & 0.37 & 33.41 & 0.44 & 25.03 & 0.32 & 9.54 & 0.20 & 13.18 & 0.25 & 11.19 & 0.22 & 15.42 & 0.30 \\
\hline $232^{\circ} \mathrm{C}$ Roll Temp & 11952 & 18.55 & 0.30 & 32.82 & 0.46 & 24.67 & 0.29 & 9.34 & 0.11 & 12.94 & 0.15 & 11.11 & 0.11 & 15.40 & 0.16 \\
\hline $246{ }^{\circ} \mathrm{C}$ Roll Temp & 11953 & 18.29 & 0.34 & 32.20 & 0.45 & 24.26 & 0.30 & 9.40 & 0.09 & 13.29 & 0.10 & 11.04 & 0.10 & 15.64 & 0.12 \\
\hline $260^{\circ} \mathrm{C}$ Roll Temp & 11954 & 18.83 & 0.34 & 32.89 & 0.40 & 24.88 & 0.31 & 9.85 & 0.09 & 13.57 & 0.11 & 11.34 & 0.10 & 15.70 & 0.12 \\
\hline $274^{\circ} \mathrm{C}$ Roll Temp & 11955 & 18.76 & 0.40 & 32.50 & 0.57 & 24.68 & 0.38 & 9.43 & 0.14 & 12.68 & 0.16 & 10.80 & 0.16 & 14.62 & 0.16 \\
\hline $288^{\circ} \mathrm{C}$ Roll Temp & 11956 & 18.60 & 0.38 & 33.50 & 0.50 & 24.96 & 0.34 & 8.72 & 0.13 & 12.06 & 0.16 & 10.11 & 0.14 & 13.92 & 0.18 \\
\hline WP - $43.8 \mathrm{KN} / \mathrm{m}$ Cal. & 11937 & & & & & & & & & & & & & & \\
\hline WP - $37.1 \mathrm{KN} / \mathrm{m} \mathrm{Cal}$. & 11938 & & & & & & & & & & & & & & \\
\hline WP - $10.5 \mathrm{KN} / \mathrm{m}$ Cal. & 11939 & & & & & & & & & & & & & & \\
\hline WP - $28.7 \mathrm{KN} / \mathrm{m} \mathrm{Cal}$ & 11940 & & & & & & & & & & & & & & \\
\hline WP - $19.8 \mathrm{KN} / \mathrm{m}$ Cal. & 11941 & & & & & & & & & & & & & & \\
\hline
\end{tabular}


Table E2 cont. Average Physical Property Data.

\begin{tabular}{|c|c|c|c|c|c|c|c|c|c|c|c|c|c|}
\hline \multirow[t]{2}{*}{ Test Method } & \multirow[b]{2}{*}{$\begin{array}{c}\text { Sample } \\
\text { ID } \\
\text { (\#) }\end{array}$} & \multicolumn{8}{|l|}{ T494 } & \multicolumn{4}{|l|}{ T494 } \\
\hline & & $\begin{array}{c}\text { CD } \\
\text { Tensile } \\
\text { (lbf/in) }\end{array}$ & $\begin{array}{l}95 \% \\
\text { C.I. }\end{array}$ & $\begin{array}{l}\text { MD } \\
\text { Tensile } \\
\text { (Ibf/in) }\end{array}$ & $\begin{array}{l}95 \% \\
\text { C.I. }\end{array}$ & $\begin{array}{l}\text { CD Tensile } \\
\text { Index } \\
\left(\mathrm{N}^{*} \mathrm{~m} / \mathrm{g}\right)\end{array}$ & $\begin{array}{c}95 \% \\
\text { C.I. }\end{array}$ & $\begin{array}{l}\text { MD Tensile } \\
\text { Index } \\
\left(\mathrm{N}^{*} \mathrm{~m} / \mathrm{g}\right)\end{array}$ & $\begin{array}{c}95 \% \\
\text { C.I. }\end{array}$ & $\begin{array}{c}C D \\
\text { Young's } \\
\text { Modulus } \\
\left(\mathrm{lbf} / \mathrm{in}^{\wedge} 2\right)\end{array}$ & $\begin{array}{l}95 \% \\
\text { C.I. }\end{array}$ & $\begin{array}{c}\text { MD } \\
\text { Young's } \\
\text { Modulus } \\
\text { (lbf/in^2) }\end{array}$ & $\begin{array}{c}95 \% \\
\text { C.I. }\end{array}$ \\
\hline Case 5 & 11942 & 23.75 & 0.41 & 59.63 & 1.13 & 27.30 & 0.50 & 68.08 & 1.27 & 20081 & 388 & 53069 & 1361 \\
\hline Wet Press & 11943 & 23.53 & 0.52 & 57.11 & 1.20 & 27.80 & 0.56 & 67.31 & 1.47 & 19107 & 405 & 51404 & 869 \\
\hline \multirow[t]{3}{*}{ No Calender } & 11944 & 24.20 & 0.42 & 60.48 & 0.97 & 27.41 & 0.45 & 68.06 & 1.10 & 20318 & 322 & 52607 & 986 \\
\hline & 11945 & 23.63 & 0.37 & 58.95 & 1.12 & 27.07 & 0.45 & 67.02 & 1.18 & 20436 & 255 & 53391 & 697 \\
\hline & Average & 23.78 & 0.22 & 59.04 & 0.55 & 27.40 & 0.25 & 67.62 & 0.63 & 19985 & 174 & 52618 & 504 \\
\hline Case 3 & 11946 & 23.74 & 0.42 & 58.77 & 1.17 & 27.26 & 0.48 & 67.89 & 1.25 & 19632 & 430 & 55338 & 2971 \\
\hline Wet Press & 11947 & 23.32 & 0.39 & 57.71 & 1.16 & 27.31 & 0.46 & 67.52 & 1.25 & 19223 & 461 & 52869 & 828 \\
\hline \multirow[t]{3}{*}{$35 \mathrm{KN} / \mathrm{m}$ Calender } & 11948 & 23.38 & 0.47 & 57.95 & 1.14 & 27.19 & 0.53 & 67.66 & 1.26 & 19229 & 267 & 51132 & 590 \\
\hline & 11949 & 23.10 & 0.42 & 58.12 & 0.94 & 26.71 & 0.47 & 67.24 & 1.18 & 18674 & 287 & 49830 & 550 \\
\hline & Average & 23.39 & 0.21 & 58.14 & 0.55 & 27.12 & 0.24 & 67.58 & 0.62 & 19189 & 186 & 52292 & 797 \\
\hline Case 2 & 11926 & 26.79 & 0.71 & 67.66 & 1.20 & 31.24 & 0.75 & 79.10 & 1.51 & 21592 & 463 & 56515 & 989 \\
\hline $246^{\circ} \mathrm{C}$ & 11930 & 27.31 & 0.67 & 67.47 & 1.23 & 31.40 & 0.74 & 77.44 & 1.50 & 21419 & 404 & 53338 & 2994 \\
\hline \multirow{3}{*}{ No Calender } & 11931 & 26.98 & 0.49 & 67.40 & 1.17 & 31.81 & 0.60 & 79.64 & 1.39 & 20462 & 451 & 54203 & 1085 \\
\hline & 11932 & 26.79 & 0.64 & 68.29 & 1.03 & 31.85 & 0.76 & 81.08 & 1.06 & 20354 & 407 & 53875 & 1073 \\
\hline & Average & 26.97 & 0.32 & 67.71 & 0.58 & 31.57 & 0.36 & 79.32 & 0.69 & 20957 & 216 & 54483 & 876 \\
\hline Case 6 & 11927 & 26.96 & 0.59 & 67.21 & 1.13 & 31.72 & 0.72 & 78.62 & 1.26 & 20997 & 394 & 55511 & 964 \\
\hline $246^{\circ} \mathrm{C}$ & 11928 & 27.20 & 0.51 & 67.10 & 1.37 & 31.53 & 0.62 & 77.78 & 1.50 & 20963 & 443 & 56186 & 942 \\
\hline $35 \mathrm{KN} / \mathrm{m}$ Calender & Average & 27.08 & 0.39 & 67.16 & 0.89 & 31.62 & 0.47 & 78.20 & 0.98 & 20980 & 296 & 55849 & 674 \\
\hline Case 4 & 11933 & 26.23 & 0.64 & 66.54 & 1.16 & 31.10 & 0.75 & 79.42 & 1.32 & 20731 & 475 & 56241 & 773 \\
\hline $260^{\circ} \mathrm{C}$ & 11934 & 27.02 & 0.80 & 68.68 & 1.05 & 31.31 & 0.82 & 79.62 & 1.16 & 19886 & 740 & 54698 & 1030 \\
\hline \multirow[t]{3}{*}{ No Calender } & 11935 & 27.33 & 0.53 & 68.81 & 1.15 & 31.55 & 0.62 & 79.39 & 1.34 & 20714 & 301 & 51786 & 685 \\
\hline & 11936 & 26.44 & 0.56 & 68.29 & 1.05 & 30.92 & 0.64 & 79.65 & 1.10 & 20634 & 335 & 52409 & 585 \\
\hline & Average & 26.75 & 0.32 & 68.08 & 0.55 & 31.22 & 0.36 & 79.52 & 0.62 & 20491 & 247 & 53784 & 393 \\
\hline Case 1 & 225111 & 26.99 & 0.55 & 75.61 & 2.22 & 32.72 & 0.71 & 91.33 & 2.28 & 18888 & 340 & 59413 & 1432 \\
\hline \multirow{2}{*}{$\begin{array}{l}\text { Commercial } \\
\text { Liner - Control }\end{array}$} & 225121 & 26.00 & 0.71 & 75.96 & 1.53 & 30.85 & 0.92 & 88.90 & 2.09 & 18140 & 753 & 53794 & 1052 \\
\hline & Average & 26.50 & 0.45 & 75.78 & 1.35 & 31.78 & 0.58 & 90.11 & 1.55 & 18514 & 413 & 56604 & 888 \\
\hline Commercial & 107811 & 12.44 & 0.20 & 37.07 & 0.66 & 18.88 & 0.25 & 55.76 & 0.88 & 10569 & 159 & 36430 & 534 \\
\hline \multirow{5}{*}{ Medium } & 107812 & 12.83 & 0.15 & 35.92 & 0.71 & 19.53 & 0.20 & 54.77 & 0.99 & 10358 & 189 & 35496 & 504 \\
\hline & 107813 & 12.03 & 0.20 & 34.77 & 1.01 & 18.48 & 0.30 & 53.26 & 1.32 & 9720 & 191 & 33436 & 727 \\
\hline & 107814 & 12.42 & 0.19 & 37.75 & 0.81 & 18.79 & 0.28 & 56.99 & 0.85 & 10163 & 164 & 36608 & 865 \\
\hline & 107816 & 12.50 & 0.19 & 36.01 & 0.89 & 19.01 & 0.29 & 54.94 & 1.19 & 10299 & 336 & 36406 & 930 \\
\hline & Average & 12.44 & 0.08 & 36.30 & 0.37 & 18.94 & 0.12 & 55.14 & 0.48 & 10222 & 97 & 35675 & 327 \\
\hline
\end{tabular}


Table E2 cont. Average Physical Property Data.

\begin{tabular}{|c|c|c|c|c|c|c|c|c|c|c|c|c|c|}
\hline \multirow[t]{2}{*}{ Test Method } & & \multicolumn{8}{|l|}{ T494 } & \multicolumn{4}{|l|}{ T494 } \\
\hline & $\begin{array}{c}\text { Sample } \\
\text { ID } \\
\text { (\#) }\end{array}$ & $\begin{array}{c}\text { CD } \\
\text { Tensile } \\
\text { (lbf/in) }\end{array}$ & $\begin{array}{l}95 \% \\
\text { C.I. }\end{array}$ & $\begin{array}{c}\text { MD } \\
\text { Tensile } \\
\text { (lbf/in) }\end{array}$ & $\begin{array}{l}95 \% \\
\text { C.I. }\end{array}$ & $\begin{array}{c}\text { CD Tensile } \\
\text { Index } \\
\left(N^{*} \mathrm{~m} / \mathrm{g}\right)\end{array}$ & $\begin{array}{l}95 \% \\
\text { C.I. }\end{array}$ & $\begin{array}{c}\text { MD Tensile } \\
\text { Index } \\
\left(N^{*} \mathrm{~m} / \mathrm{g}\right)\end{array}$ & $\begin{array}{l}95 \% \\
\text { C.I. }\end{array}$ & $\begin{array}{c}\text { CD } \\
\text { Young's } \\
\text { Modulus } \\
\left(\mathrm{lbf}^{\prime} / \mathrm{in}^{\wedge} 2\right)\end{array}$ & $\begin{array}{l}95 \% \\
\text { C.I. }\end{array}$ & $\begin{array}{c}\text { MD } \\
\text { Young's } \\
\text { Modulus } \\
(\text { lbf/in^2) }\end{array}$ & $\begin{array}{c}95 \% \\
\text { C.I. }\end{array}$ \\
\hline $204^{\circ} \mathrm{C}$ Roll Temp & 11950 & 24.71 & $0 . \overline{44}$ & 60.90 & 2.50 & 28.98 & 0.55 & 71.38 & 2.91 & 20659 & 224 & 51581 & 1249 \\
\hline $218^{\circ} \mathrm{C}$ Roll Temp & 11951 & 25.03 & 0.53 & 61.59 & 1.08 & 29.34 & 0.61 & 72.03 & 1.22 & 19312 & 503 & 52750 & 1360 \\
\hline $232^{\circ} \mathrm{C}$ Roll Temp & 11952 & 24.66 & 0.46 & 62.08 & 1.03 & 29.24 & 0.55 & 73.93 & 1.22 & 20472 & 237 & 53513 & 1057 \\
\hline $246^{\circ} \mathrm{C}$ Roll Temp & 11953 & 24.95 & 0.47 & 62.08 & 1.00 & 29.49 & 0.56 & 73.87 & 1.12 & 20081 & 276 & 52563 & 682 \\
\hline $260^{\circ} \mathrm{C}$ Roll Temp & 11954 & 25.61 & 0.54 & 63.89 & 1.40 & 29.73 & 0.60 & 74.07 & 1.59 & 20668 & 368 & 52402 & 2542 \\
\hline $274^{\circ} \mathrm{C}$ Roll Temp & 11955 & 25.69 & 0.54 & 63.81 & 0.96 & 29.57 & 0.61 & 73.64 & 1.18 & 20171 & 346 & 53672 & 825 \\
\hline $288^{\circ} \mathrm{C}$ Roll Temp & 11956 & 25.90 & 0.46 & 64.08 & 1.19 & 30.36 & 0.51 & 74.62 & 1.27 & 19638 & 343 & 53220 & 669 \\
\hline WP $-43.8 \mathrm{KN} / \mathrm{m} \mathrm{Cal}$. & 11937 & & & & & & & & & & & & \\
\hline WP - 37.1 KN/m Cal. & 11938 & & & & & & & & & & & & \\
\hline WP - $10.5 \mathrm{KN} / \mathrm{m} \mathrm{Cal}$. & 11939 & & & & & & & & & & & & \\
\hline WP - $28.7 \mathrm{KN} / \mathrm{m} \mathrm{Cal}$. & 11940 & & & & & & & & & & & & \\
\hline $\mathrm{WP}-19.8 \mathrm{KN} / \mathrm{m} \mathrm{Cal}$. & 11941 & & & & & & & & & & & & \\
\hline
\end{tabular}


Table E2 cont. Average Physical Property Data.

\begin{tabular}{|c|c|c|c|c|c|c|c|c|c|c|c|c|c|}
\hline \multicolumn{2}{|l|}{ Test Method } & & & & & \multicolumn{2}{|l|}{ IPST } & \multicolumn{2}{|l|}{ IPST } & \multicolumn{4}{|l|}{ T809 } \\
\hline & $\begin{array}{c}\text { Sample } \\
\text { ID } \\
\text { (\#) }\end{array}$ & $\begin{array}{c}\mathrm{CD} \\
\text { Young's } \\
\text { Modulus } \\
\left(\mathrm{MN} / \mathrm{m}^{\wedge} 2\right)\end{array}$ & $\begin{array}{l}95 \% \\
\text { C.I. }\end{array}$ & $\begin{array}{c}\text { MD } \\
\text { Young's } \\
\text { Modulus } \\
\left(\mathrm{MN} / \mathrm{m}^{\wedge} 2\right)\end{array}$ & $\begin{array}{c}95 \% \\
\text { C.I. }\end{array}$ & $\begin{array}{c}\text { OD Basis } \\
\text { Weight } \\
\left(\mathrm{g} / \mathrm{m}^{\wedge} 2\right)\end{array}$ & $\begin{array}{l}95 \% \\
\text { C.I. }\end{array}$ & $\begin{array}{c}\text { OD } \\
\text { Density } \\
\left(\mathrm{g} / \mathrm{cm}^{\wedge} 3\right)\end{array}$ & $\begin{array}{l}95 \% \\
\text { C.I. }\end{array}$ & $\begin{array}{l}\text { Concora } \\
\text { (lbf) }\end{array}$ & $\begin{array}{l}95 \% \\
\text { C.I. }\end{array}$ & $\begin{array}{c}\text { Concora } \\
\text { (N) }\end{array}$ & $\begin{array}{l}95 \% \\
\text { C.I. }\end{array}$ \\
\hline Case 5 & 11942 & 138.4 & 2.7 & 365.9 & 9.4 & 152.0 & 0.4 & 0.624 & 0.007 & & & & \\
\hline Wet Press & 11943 & 131.7 & 2.8 & 354.4 & 6.0 & 148.1 & 0.4 & 0.622 & 0.007 & & & & \\
\hline \multirow{3}{*}{ No Calender } & 11944 & 140.1 & 2.2 & 362.7 & 6.8 & 154.7 & 0.4 & 0.627 & 0.008 & & & & \\
\hline & 11945 & 140.9 & 1.8 & 368.1 & 4.8 & 153.8 & 0.4 & 0.615 & 0.007 & & & & \\
\hline & Average & 137.8 & 1.2 & 362.8 & 3.5 & 152.1 & 0.2 & 0.622 & 0.004 & & & & \\
\hline Case 3 & 11946 & 135.3 & 3.0 & 381.5 & 20.5 & 151.3 & 0.3 & 0.634 & 0.005 & & & & \\
\hline Wet Press & 11947 & 132.5 & 3.2 & 364.5 & 5.7 & 150.0 & 0.3 & 0.634 & 0.005 & & & & \\
\hline \multirow[t]{3}{*}{$35 \mathrm{KN} / \mathrm{m}$ Calender } & 11948 & 132.6 & 1.8 & 352.5 & 4.1 & 150.0 & 0.4 & 0.631 & 0.007 & & & & \\
\hline & 11949 & 128.7 & 2.0 & 343.5 & 3.8 & 151.1 & 0.4 & 0.632 & 0.007 & & & & \\
\hline & Average & 132.3 & 1.3 & 360.5 & 5.5 & 150.6 & 0.2 & 0.633 & 0.003 & & & & \\
\hline Case 2 & 11926 & 148.9 & 3.2 & 389.6 & 6.8 & 150.0 & 0.3 & 0.693 & 0.010 & & & & \\
\hline $246^{\circ} \mathrm{C}$ & 11930 & 147.7 & 2.8 & 367.7 & 20.6 & 152.5 & 0.4 & 0.698 & 0.011 & & & & \\
\hline \multirow[t]{3}{*}{ No Calender } & 11931 & 141.1 & 3.1 & 373.7 & 7.5 & 148.9 & 0.4 & 0.687 & 0.010 & & & & \\
\hline & 11932 & 140.3 & 2.8 & 371.4 & 7.4 & 147.7 & 0.4 & 0.680 & 0.016 & & & & \\
\hline & Average & 144.5 & 1.5 & 375.6 & 6.0 & 149.8 & 0.2 & 0.689 & 0.006 & & & & \\
\hline Case 6 & 11927 & 144.8 & 2.7 & 382.7 & 6.6 & 149.4 & 0.3 & 0.728 & 0.006 & & & & \\
\hline $246^{\circ} \mathrm{C}$ & 11928 & 144.5 & 3.1 & 387.4 & 6.5 & 150.9 & 0.3 & 0.715 & 0.007 & & & & \\
\hline $35 \mathrm{KN} / \mathrm{m}$ Calender & Average & 144.6 & 2.0 & 385.0 & 4.6 & 150.1 & 0.2 & 0.721 & 0.005 & & & & \\
\hline Case 4 & 11933 & 142.9 & 3.3 & 387.7 & 5.3 & 147.3 & 0.4 & 0.685 & 0.012 & & & & \\
\hline $260^{\circ} \mathrm{C}$ & 11934 & 137.1 & 5.1 & 377.1 & 7.1 & 150.6 & 0.4 & 0.672 & 0.011 & & & & \\
\hline \multirow[t]{3}{*}{ No Calender } & 11935 & 142.8 & 2.1 & 357.0 & 4.7 & 152.0 & 0.3 & 0.694 & 0.009 & & & & \\
\hline & 11936 & 142.3 & 2.3 & 361.3 & 4.0 & 150.0 & 0.3 & 0.701 & 0.010 & & & & \\
\hline & Average & 141.3 & 1.7 & 370.8 & 2.7 & 150.0 & 0.2 & 0.688 & 0.005 & & & & \\
\hline Case 1 & 225111 & 130.2 & 2.3 & 409.6 & 9.9 & 146.4 & 0.6 & 0.701 & 0.008 & & & & \\
\hline \multirow{2}{*}{$\begin{array}{l}\text { Commercial } \\
\text { Liner - Control }\end{array}$} & 225121 & 125.1 & 5.2 & 370.9 & 7.3 & 147.1 & 9.4 & 0.698 & 0.046 & & & & \\
\hline & Average & 127.6 & 2.8 & 390.2 & 6.1 & 146.8 & 4.7 & 0.700 & 0.023 & & & & \\
\hline Commercial & 107811 & 72.9 & 1.1 & 251.2 & 3.7 & 116.5 & 0.4 & 0.616 & 0.007 & 49.83 & 1.08 & 221.7 & 4.8 \\
\hline \multirow[t]{5}{*}{ Medium } & 107812 & 71.4 & 1.3 & 244.7 & 3.5 & 115.0 & 1.9 & 0.631 & 0.012 & 48.72 & 0.92 & 216.7 & 4.1 \\
\hline & 107813 & 67.0 & 1.3 & 230.5 & 5.0 & 114.1 & 0.4 & 0.633 & 0.007 & 49.40 & 1.29 & 219.7 & 5.7 \\
\hline & 107814 & 70.1 & 1.1 & 252.4 & 6.0 & 116.4 & 0.5 & 0.613 & 0.007 & 48.30 & 1.41 & 214.8 & 6.3 \\
\hline & 107816 & 71.0 & 2.3 & 251.0 & 6.4 & 114.8 & 0.4 & 0.620 & 0.007 & 51.13 & 1.40 & 227.4 & 6.2 \\
\hline & Average & 70.5 & 0.7 & 246.0 & 2.3 & 115.4 & 0.4 & 0.623 & 0.004 & 49.48 & 0.55 & 220.1 & 2.5 \\
\hline
\end{tabular}


Table E2 cont. Average Physical Property Data.

\begin{tabular}{|c|c|c|c|c|c|c|c|c|c|c|c|c|c|}
\hline \multicolumn{2}{|l|}{ Test Method } & & & & & \multicolumn{2}{|l|}{ IPST } & \multicolumn{2}{|l|}{ IPST } & \multicolumn{4}{|l|}{ T809 } \\
\hline & $\begin{array}{c}\text { Sample } \\
\text { ID } \\
(\#)\end{array}$ & $\begin{array}{c}\mathrm{CD} \\
\text { Young's } \\
\text { Modulus } \\
\left(\mathrm{MN} / \mathrm{m}^{\wedge} 2\right)\end{array}$ & $\begin{array}{l}95 \% \\
\text { C.I. }\end{array}$ & $\begin{array}{c}\text { MD } \\
\text { Young's } \\
\text { Modulus } \\
\left(\mathrm{MN} / \mathrm{m}^{\wedge} 2\right)\end{array}$ & $\begin{array}{c}95 \% \\
\text { C.I. }\end{array}$ & $\begin{array}{c}\text { OD Basis } \\
\text { Weight } \\
\left(\mathrm{g} / \mathrm{m}^{\wedge} 2\right)\end{array}$ & $\begin{array}{l}95 \% \\
\text { C.I. }\end{array}$ & $\begin{array}{c}\text { OD } \\
\text { Density } \\
\left(\mathrm{g} / \mathrm{cm}^{\wedge} 3\right)\end{array}$ & $\begin{array}{c}95 \% \\
\text { C.I. }\end{array}$ & $\begin{array}{c}\text { Concora } \\
\text { (lbf) }\end{array}$ & $\begin{array}{l}95 \% \\
\text { C.I. }\end{array}$ & $\begin{array}{l}\text { Concora } \\
\text { (N) }\end{array}$ & $\begin{array}{l}95 \% \\
\text { C.I. }\end{array}$ \\
\hline $204^{\circ} \mathrm{C}$ Roll Temp & 11950 & 142.4 & 1.5 & 355.6 & 8.6 & 149.6 & $\overline{0.3}$ & 0.651 & 0.007 & & & & \\
\hline $218^{\circ} \mathrm{C}$ Roll Temp & 11951 & 133.1 & 3.5 & 363.7 & 9.4 & 149.5 & 0.3 & 0.678 & 0.008 & & & & \\
\hline $232^{\circ} \mathrm{C}$ Roll Temp & 11952 & 141.1 & 1.6 & 368.9 & 7.3 & 147.1 & 0.2 & 0.684 & 0.008 & & & & \\
\hline $246^{\circ} \mathrm{C}$ Roll Temp & 11953 & 138.4 & 1.9 & 362.4 & 4.7 & 149.1 & 0.3 & 0.684 & 0.008 & & & & \\
\hline $260^{\circ} \mathrm{C}$ Roll Temp & 11954 & 142.5 & 2.5 & 361.3 & 17.5 & 151.6 & 0.3 & 0.686 & 0.008 & & & & \\
\hline $274^{\circ} \mathrm{C}$ Roll Temp & 11955 & 139.1 & 2.4 & 370.0 & 5.7 & 152.3 & 0.3 & 0.654 & 0.009 & & & & \\
\hline $288^{\circ} \mathrm{C}$ Roll Temp & 11956 & 135.4 & 2.4 & 366.9 & 4.6 & 150.8 & 0.3 & 0.669 & 0.009 & & & & \\
\hline $\begin{array}{l}\text { WP - 43.8 KN/m Cal. } \\
\text { WP - 37.1 KN/m Cal. } \\
\text { WP - 10.5 KN/m Cal. } \\
\text { WP - 28.7 KN/m Cal. } \\
\text { WP - 19.8 KN/m Cal. }\end{array}$ & $\begin{array}{l}11937 \\
11938 \\
11939 \\
11940 \\
11941 \\
\end{array}$ & & & & & & & & & & & & \\
\hline
\end{tabular}


Table E3. Average Physical Property Data by Lane.

\begin{tabular}{|c|c|c|c|c|c|c|c|c|c|c|c|c|c|c|}
\hline Test Methods & IPST & & IPST & & & & $\mathrm{T} 460$ & & UM535 & & & & T538 & \\
\hline $\begin{array}{l}\text { Sample } \\
\text { ID } \\
\text { (\#) }\end{array}$ & $\begin{array}{l}\text { ZD-Long. } \\
\text { SEM } \\
\text { (MN m/kg) }\end{array}$ & $\begin{array}{l}95 \% \\
\text { C.I. }\end{array}$ & $\begin{array}{c}\text { Soft } \\
\text { Caliper } \\
\text { (microns) }\end{array}$ & $\begin{array}{c}95 \% \\
\text { C.I. }\end{array}$ & $\begin{array}{c}\text { Soft } \\
\text { Caliper } \\
\text { (mills) }\end{array}$ & $\begin{array}{l}95 \% \\
\text { C.I. }\end{array}$ & $\begin{array}{c}\text { Gurley } \\
\text { Porosity } \\
\text { (s/100ml) }\end{array}$ & $\begin{array}{l}95 \% \\
\text { C.I. }\end{array}$ & $\begin{array}{c}\text { FS } \\
\text { Bendtsen } \\
\text { Roughness } \\
\text { (ml/min) }\end{array}$ & $\begin{array}{l}95 \% \\
\text { C.II. }\end{array}$ & $\begin{array}{c}\text { WS } \\
\text { Bendtsen } \\
\text { Roughness } \\
\text { (ml/min) }\end{array}$ & $\begin{array}{c}95 \% \\
\text { C.I. }\end{array}$ & $\begin{array}{c}\text { FS } \\
\text { Sheffield } \\
\text { Roughness } \\
\text { (SU) }\end{array}$ & $\begin{array}{l}95 \% \\
\text { C.I. }\end{array}$ \\
\hline $\begin{array}{l}11926 \\
\text { Front } \\
\text { Center } \\
\text { Back }\end{array}$ & $\begin{array}{l}0.1398 \\
0.1229 \\
0.1363\end{array}$ & $\begin{array}{l}0.0050 \\
0.0029 \\
0.0050\end{array}$ & $\begin{array}{l}218.47 \\
217.23 \\
224.67\end{array}$ & $\begin{array}{l}5.00 \\
4.66 \\
7.05\end{array}$ & $\begin{array}{l}8.60 \\
8.55 \\
8.85\end{array}$ & $\begin{array}{l}0.20 \\
0.18 \\
0.28\end{array}$ & $\begin{array}{l}24.96 \\
21.30 \\
23.44\end{array}$ & $\begin{array}{l}0.46 \\
0.46 \\
0.64\end{array}$ & $\begin{array}{l}1816 \\
1908 \\
1916\end{array}$ & $\begin{array}{l}105 \\
139 \\
111\end{array}$ & $\begin{array}{l}2343 \\
2284 \\
2282\end{array}$ & $\begin{array}{l}105 \\
100 \\
114\end{array}$ & $\begin{array}{l}402 \\
404 \\
403\end{array}$ & $\begin{array}{l}7 \\
9 \\
7\end{array}$ \\
\hline $\begin{array}{l}11927 \\
\text { Front } \\
\text { Center } \\
\text { Back }\end{array}$ & $\begin{array}{l}0.1288 \\
0.1036 \\
0.1129\end{array}$ & $\begin{array}{l}0.0048 \\
0.0034 \\
0.0038\end{array}$ & $\begin{array}{l}205.20 \\
206.17 \\
207.27 \\
\end{array}$ & $\begin{array}{l}2.11 \\
2.36 \\
2.68\end{array}$ & $\begin{array}{l}8.08 \\
8.12 \\
8.16\end{array}$ & $\begin{array}{l}0.08 \\
0.09 \\
0.11\end{array}$ & $\begin{array}{l}25.91 \\
22.35 \\
24.92 \\
\end{array}$ & $\begin{array}{l}0.95 \\
0.53 \\
0.82 \\
\end{array}$ & $\begin{array}{l}1506 \\
1150 \\
1269 \\
\end{array}$ & $\begin{array}{l}107 \\
106 \\
139 \\
\end{array}$ & $\begin{array}{l}1859 \\
1723 \\
1749 \\
\end{array}$ & $\begin{array}{c}80 \\
82 \\
118 \\
\end{array}$ & $\begin{array}{l}376 \\
350 \\
365 \\
\end{array}$ & $\begin{array}{l}11 \\
10 \\
10 \\
\end{array}$ \\
\hline $\begin{array}{l}11928 \\
\text { Front } \\
\text { Center } \\
\text { Back }\end{array}$ & $\begin{array}{l}0.1360 \\
0.1099 \\
0.1190\end{array}$ & $\begin{array}{l}0.0069 \\
0.0049 \\
0.0051\end{array}$ & $\begin{array}{l}210.83 \\
209.57 \\
210.97\end{array}$ & $\begin{array}{l}3.10 \\
2.17 \\
3.52\end{array}$ & $\begin{array}{l}8.30 \\
8.25 \\
8.31\end{array}$ & $\begin{array}{l}0.12 \\
0.09 \\
0.14\end{array}$ & $\begin{array}{l}26.21 \\
24.48 \\
25.66\end{array}$ & $\begin{array}{l}1.00 \\
0.99 \\
0.99 \\
\end{array}$ & $\begin{array}{l}1430 \\
1105 \\
1243 \\
\end{array}$ & $\begin{array}{c}102 \\
84 \\
95 \\
\end{array}$ & $\begin{array}{l}1770 \\
1782 \\
1651 \\
\end{array}$ & $\begin{array}{l}87 \\
61 \\
78 \\
\end{array}$ & $\begin{array}{l}378 \\
350 \\
356 \\
\end{array}$ & $\begin{array}{c}7 \\
11 \\
8 \\
\end{array}$ \\
\hline $\begin{array}{l}11930 \\
\text { Front } \\
\text { Center } \\
\text { Back }\end{array}$ & $\begin{array}{l}0.1469 \\
0.1298 \\
0.1391\end{array}$ & $\begin{array}{l}0.0046 \\
0.0035 \\
0.0044\end{array}$ & $\begin{array}{l}210.20 \\
222.13 \\
221.47 \\
\end{array}$ & $\begin{array}{l}3.33 \\
5.72 \\
4.43 \\
\end{array}$ & $\begin{array}{l}8.28 \\
8.75 \\
8.72 \\
\end{array}$ & $\begin{array}{l}0.13 \\
0.23 \\
0.17 \\
\end{array}$ & $\begin{array}{l}23.69 \\
20.92 \\
22.58 \\
\end{array}$ & $\begin{array}{l}0.52 \\
0.35 \\
0.55 \\
\end{array}$ & $\begin{array}{l}1927 \\
1749 \\
1795 \\
\end{array}$ & $\begin{array}{c}151 \\
142 \\
98 \\
\end{array}$ & $\begin{array}{l}2390 \\
2543 \\
2422 \\
\end{array}$ & $\begin{array}{l}116 \\
102 \\
94 \\
\end{array}$ & $\begin{array}{l}392 \\
387 \\
394 \\
\end{array}$ & $\begin{array}{l}7 \\
7 \\
6 \\
\end{array}$ \\
\hline $\begin{array}{l}11931 \\
\text { Front } \\
\text { Center } \\
\text { Back } \\
\end{array}$ & $\begin{array}{l}0.1442 \\
0.1294 \\
0.1400 \\
\end{array}$ & $\begin{array}{l}0.0045 \\
0.0035 \\
0.0039 \\
\end{array}$ & $\begin{array}{l}206.93 \\
220.27 \\
216.77 \\
\end{array}$ & $\begin{array}{c}13.56 \\
5.15 \\
4.31 \\
\end{array}$ & $\begin{array}{l}8.15 \\
8.67 \\
8.53 \\
\end{array}$ & $\begin{array}{l}0.53 \\
0.20 \\
0.17 \\
\end{array}$ & $\begin{array}{l}24.61 \\
21.75 \\
24.41 \\
\end{array}$ & $\begin{array}{l}0.86 \\
0.55 \\
0.77 \\
\end{array}$ & $\begin{array}{l}1931 \\
1823 \\
1876 \\
\end{array}$ & $\begin{array}{l}112 \\
129 \\
134 \\
\end{array}$ & $\begin{array}{l}2220 \\
2377 \\
2171 \\
\end{array}$ & $\begin{array}{c}91 \\
106 \\
93 \\
\end{array}$ & $\begin{array}{l}403 \\
396 \\
398 \\
\end{array}$ & $\begin{array}{l}8 \\
5 \\
7 \\
\end{array}$ \\
\hline $\begin{array}{l}11932 \\
\text { Front } \\
\text { Center } \\
\text { Back }\end{array}$ & $\begin{array}{l}0.1444 \\
0.1271 \\
0.1362\end{array}$ & $\begin{array}{l}0.0047 \\
0.0035 \\
0.0051\end{array}$ & $\begin{array}{l}217.73 \\
212.87 \\
223.03\end{array}$ & $\begin{array}{l}7.87 \\
7.78 \\
4.27\end{array}$ & $\begin{array}{l}8.57 \\
8.38 \\
8.78\end{array}$ & $\begin{array}{l}0.31 \\
0.31 \\
0.17\end{array}$ & $\begin{array}{l}24.31 \\
21.12 \\
23.44\end{array}$ & $\begin{array}{l}0.58 \\
0.36 \\
0.60\end{array}$ & $\begin{array}{l}1967 \\
1850 \\
1886\end{array}$ & $\begin{array}{c}83 \\
99 \\
109\end{array}$ & $\begin{array}{l}2143 \\
2294 \\
2196\end{array}$ & $\begin{array}{c}92 \\
118 \\
78\end{array}$ & $\begin{array}{l}400 \\
402 \\
399\end{array}$ & $\begin{array}{l}8 \\
7 \\
6\end{array}$ \\
\hline $\begin{array}{l}11933 \\
\text { Front } \\
\text { Center } \\
\text { Back } \\
\end{array}$ & $\begin{array}{l}0.1428 \\
0.1225 \\
0.1396 \\
\end{array}$ & $\begin{array}{l}0.0053 \\
0.0032 \\
0.0046 \\
\end{array}$ & $\begin{array}{l}210.67 \\
216.47 \\
219.50 \\
\end{array}$ & $\begin{array}{l}5.11 \\
6.04 \\
4.77\end{array}$ & $\begin{array}{l}8.29 \\
8.52 \\
8.68\end{array}$ & $\begin{array}{l}0.20 \\
0.24 \\
0.18 \\
\end{array}$ & $\begin{array}{l}23.68 \\
20.14 \\
22.44 \\
\end{array}$ & $\begin{array}{l}0.67 \\
0.28 \\
0.57 \\
\end{array}$ & $\begin{array}{l}1887 \\
1876 \\
1965 \\
\end{array}$ & $\begin{array}{l}129 \\
119 \\
137 \\
\end{array}$ & $\begin{array}{l}2120 \\
2141 \\
2069 \\
\end{array}$ & $\begin{array}{c}111 \\
97 \\
80 \\
\end{array}$ & $\begin{array}{l}399 \\
394 \\
399 \\
\end{array}$ & $\begin{array}{l}8 \\
7 \\
7 \\
\end{array}$ \\
\hline $\begin{array}{l}11934 \\
\text { Front } \\
\text { Center } \\
\text { Back }\end{array}$ & $\begin{array}{l}0.1403 \\
0.1269 \\
0.1357\end{array}$ & $\begin{array}{l}0.0053 \\
0.0032 \\
0.0043\end{array}$ & $\begin{array}{l}220.43 \\
228.43 \\
223.20\end{array}$ & $\begin{array}{l}6.55 \\
4.91 \\
4.27\end{array}$ & $\begin{array}{l}8.68 \\
8.99 \\
8.79\end{array}$ & $\begin{array}{l}0.26 \\
0.19 \\
0.17\end{array}$ & $\begin{array}{l}23.48 \\
20.44 \\
22.41\end{array}$ & $\begin{array}{l}0.69 \\
0.37 \\
0.58\end{array}$ & $\begin{array}{l}1988 \\
1859 \\
1914\end{array}$ & $\begin{array}{l}149 \\
113 \\
115\end{array}$ & $\begin{array}{l}2203 \\
2352 \\
2196\end{array}$ & $\begin{array}{c}102 \\
120 \\
72\end{array}$ & $\begin{array}{l}401 \\
398 \\
397\end{array}$ & $\begin{array}{c}9 \\
7 \\
10 \\
\end{array}$ \\
\hline
\end{tabular}


Table E3 cont. Average Physical Property Data by Lane.

\begin{tabular}{|c|c|c|c|c|c|c|c|c|c|c|c|c|c|c|}
\hline Test Methods & IPST & & IPST & & & & $\mathrm{T} 460$ & & UM535 & & & & T538 & \\
\hline $\begin{array}{l}\text { Sample } \\
\text { ID } \\
(\#)\end{array}$ & $\begin{array}{l}\text { ZD-Long. } \\
\text { SEM } \\
\text { (MN m/kg) }\end{array}$ & $\begin{array}{l}95 \% \\
\text { C.I. }\end{array}$ & $\begin{array}{c}\text { Soft } \\
\text { Caliper } \\
\text { (microns) }\end{array}$ & $\begin{array}{l}95 \% \\
\text { C.I. }\end{array}$ & $\begin{array}{c}\text { Soft } \\
\text { Caliper } \\
\text { (mills) }\end{array}$ & $\begin{array}{l}95 \% \\
\text { C.I. }\end{array}$ & $\begin{array}{c}\text { Gurley } \\
\text { Porosity } \\
(\mathrm{s} / 100 \mathrm{ml}) \\
\end{array}$ & $\begin{array}{l}95 \% \\
\text { C.I. }\end{array}$ & $\begin{array}{c}\text { FS } \\
\text { Bendtsen } \\
\text { Roughness } \\
(\mathrm{ml} / \mathrm{min}) \\
\end{array}$ & $\begin{array}{c}95 \% \\
\text { C.I. } \\
\end{array}$ & $\begin{array}{c}\text { WS } \\
\text { Bendtsen } \\
\text { Roughness } \\
\text { (ml/min) }\end{array}$ & $\begin{array}{l}95 \% \\
\text { C.I. }\end{array}$ & $\begin{array}{c}\text { FS } \\
\text { Sheffield } \\
\text { Roughness } \\
\text { (SU) } \\
\end{array}$ & $\begin{array}{l}95 \% \\
\text { C.I. }\end{array}$ \\
\hline $\begin{array}{c}11935 \\
\text { Front } \\
\text { Center } \\
\text { Back }\end{array}$ & $\begin{array}{l}0.1407 \\
0.1276 \\
0.1349\end{array}$ & $\begin{array}{l}0.0038 \\
0.0035 \\
0.0040 \\
\end{array}$ & $\begin{array}{l}214.90 \\
221.13 \\
225.97 \\
\end{array}$ & $\begin{array}{l}4.44 \\
4.29 \\
3.70 \\
\end{array}$ & $\begin{array}{l}8.46 \\
8.71 \\
8.90 \\
\end{array}$ & $\begin{array}{l}0.17 \\
0.17 \\
0.15 \\
\end{array}$ & $\begin{array}{l}25.01 \\
20.87 \\
22.51 \\
\end{array}$ & $\begin{array}{l}0.65 \\
0.34 \\
1.50 \\
\end{array}$ & $\begin{array}{l}1844 \\
1903 \\
1923 \\
\end{array}$ & $\begin{array}{l}101 \\
133 \\
108 \\
\end{array}$ & $\begin{array}{l}2214 \\
2419 \\
2258 \\
\end{array}$ & $\begin{array}{c}94 \\
104 \\
81 \\
\end{array}$ & $\begin{array}{l}401 \\
397 \\
406 \\
\end{array}$ & $\begin{array}{c}4 \\
11 \\
6 \\
\end{array}$ \\
\hline $\begin{array}{l}11936 \\
\text { Front } \\
\text { Center } \\
\text { Back }\end{array}$ & $\begin{array}{l}0.1459 \\
0.1310 \\
0.1368\end{array}$ & $\begin{array}{l}0.0043 \\
0.0040 \\
0.0039\end{array}$ & $\begin{array}{l}209.40 \\
213.30 \\
213.47 \\
\end{array}$ & $\begin{array}{l}4.67 \\
3.34 \\
4.46\end{array}$ & $\begin{array}{l}8.24 \\
8.40 \\
8.40\end{array}$ & $\begin{array}{l}0.18 \\
0.13 \\
0.18\end{array}$ & $\begin{array}{l}24.05 \\
20.58 \\
22.27\end{array}$ & $\begin{array}{l}0.60 \\
0.37 \\
0.59 \\
\end{array}$ & $\begin{array}{l}1933 \\
1717 \\
1927 \\
\end{array}$ & $\begin{array}{c}119 \\
88 \\
138 \\
\end{array}$ & $\begin{array}{l}2154 \\
2335 \\
2220 \\
\end{array}$ & $\begin{array}{c}94 \\
104 \\
103 \\
\end{array}$ & $\begin{array}{l}399 \\
395 \\
404 \\
\end{array}$ & $\begin{array}{l}8 \\
9 \\
8 \\
\end{array}$ \\
\hline $\begin{array}{l}11937 \\
\text { Front } \\
\text { Center } \\
\text { Back }\end{array}$ & & & & & & & & & $\begin{array}{l}1587 \\
1252 \\
1298\end{array}$ & $\begin{array}{l}140 \\
117 \\
137\end{array}$ & $\begin{array}{l}1621 \\
1428 \\
1320\end{array}$ & $\begin{array}{l}140 \\
248 \\
113\end{array}$ & & \\
\hline $\begin{array}{l}11938 \\
\text { Front } \\
\text { Center } \\
\text { Back }\end{array}$ & & & & & & & & & $\begin{array}{l}1666 \\
1462 \\
1655\end{array}$ & $\begin{array}{l}138 \\
173 \\
203\end{array}$ & $\begin{array}{l}1689 \\
1485 \\
1734\end{array}$ & $\begin{array}{l}128 \\
113 \\
174\end{array}$ & & \\
\hline $\begin{array}{l}11939 \\
\text { Front } \\
\text { Center } \\
\text { Back }\end{array}$ & & & & & & & & & $\begin{array}{l}1723 \\
2131 \\
2420 \\
\end{array}$ & $\begin{array}{c}146 \\
137 \\
87 \\
\end{array}$ & $\begin{array}{l}1638 \\
2114 \\
2392 \\
\end{array}$ & $\begin{array}{l}188 \\
157 \\
181 \\
\end{array}$ & & \\
\hline $\begin{array}{c}11940 \\
\text { Front } \\
\text { Center } \\
\text { Back }\end{array}$ & & & & & & & & & $\begin{array}{l}1581 \\
2177 \\
2488 \\
\end{array}$ & $\begin{array}{c}138 \\
78 \\
264 \\
\end{array}$ & $\begin{array}{l}1559 \\
2267 \\
2182 \\
\end{array}$ & $\begin{array}{l}111 \\
264 \\
179 \\
\end{array}$ & & \\
\hline $\begin{array}{c}11941 \\
\text { Front } \\
\text { Center } \\
\text { Back }\end{array}$ & & & & & & & & & $\begin{array}{l}1990 \\
2375 \\
2449 \\
\end{array}$ & $\begin{array}{l}133 \\
143 \\
165 \\
\end{array}$ & $\begin{array}{l}1774 \\
2018 \\
2239 \\
\end{array}$ & $\begin{array}{l}163 \\
157 \\
164 \\
\end{array}$ & & \\
\hline $\begin{array}{l}11942 \\
\text { Front } \\
\text { Center } \\
\text { Back }\end{array}$ & $\begin{array}{l}0.1031 \\
0.0947 \\
0.1060\end{array}$ & $\begin{array}{l}0.0029 \\
0.0024 \\
0.0038\end{array}$ & $\begin{array}{l}241.17 \\
245.93 \\
244.63\end{array}$ & $\begin{array}{l}3.49 \\
4.39 \\
3.90\end{array}$ & $\begin{array}{l}9.49 \\
9.68 \\
9.63\end{array}$ & $\begin{array}{l}0.14 \\
0.17 \\
0.15\end{array}$ & $\begin{array}{l}9.79 \\
8.83 \\
9.13\end{array}$ & $\begin{array}{l}0.24 \\
0.16 \\
0.22\end{array}$ & $\begin{array}{l}2307 \\
2245 \\
2366\end{array}$ & $\begin{array}{l}67 \\
68 \\
78\end{array}$ & $\begin{array}{l}2377 \\
2315 \\
2400\end{array}$ & $\begin{array}{l}84 \\
90 \\
95\end{array}$ & $\begin{array}{l}418 \\
412 \\
418 \\
\end{array}$ & $\begin{array}{l}5 \\
6 \\
5 \\
\end{array}$ \\
\hline
\end{tabular}


Table E3 cont. Average Physical Property Data by Lane.

\begin{tabular}{|c|c|c|c|c|c|c|c|c|c|c|c|c|c|c|}
\hline Test Methods & IPST & & IPST & & & & $\mathrm{T} 460$ & & UM535 & & & & T538 & \\
\hline $\begin{array}{c}\text { Sample } \\
\text { ID } \\
(\#) \\
\end{array}$ & $\begin{array}{l}\text { ZD-Long. } \\
\text { SEM } \\
\text { (MN m/kg) }\end{array}$ & $\begin{array}{l}95 \% \\
\text { C.I. }\end{array}$ & $\begin{array}{c}\text { Soft } \\
\text { Caliper } \\
\text { (microns) }\end{array}$ & $\begin{array}{c}95 \% \\
\text { C.I. }\end{array}$ & $\begin{array}{c}\text { Soft } \\
\text { Caliper } \\
\text { (mills) }\end{array}$ & $\begin{array}{c}95 \% \\
\text { C.I. }\end{array}$ & $\begin{array}{c}\text { Gurley } \\
\text { Porosity } \\
\text { (s/100ml) }\end{array}$ & $\begin{array}{c}95 \% \\
\text { C.I. }\end{array}$ & $\begin{array}{c}\text { FS } \\
\text { Bendtsen } \\
\text { Roughness } \\
\text { (ml/min) }\end{array}$ & $\begin{array}{l}95 \% \\
\text { C.I. }\end{array}$ & $\begin{array}{c}\text { WS } \\
\text { Bendtsen } \\
\text { Roughness } \\
(\mathrm{ml} / \mathrm{min})\end{array}$ & $\begin{array}{c}95 \% \\
\text { C.I. }\end{array}$ & $\begin{array}{c}\text { FS } \\
\text { Sheffield } \\
\text { Roughness } \\
\text { (SU) }\end{array}$ & $\begin{array}{c}95 \% \\
\text { C.I. }\end{array}$ \\
\hline $\begin{array}{c}11943 \\
\text { Front } \\
\text { Center } \\
\text { Back }\end{array}$ & $\begin{array}{l}0.1004 \\
0.0938 \\
0.0975\end{array}$ & $\begin{array}{l}0.0031 \\
0.0028 \\
0.0031\end{array}$ & $\begin{array}{l}241.27 \\
239.47 \\
237.77\end{array}$ & $\begin{array}{l}3.85 \\
2.86 \\
5.19\end{array}$ & $\begin{array}{l}9.50 \\
9.43 \\
9.36\end{array}$ & $\begin{array}{l}0.15 \\
0.11 \\
0.20\end{array}$ & $\begin{array}{l}9.76 \\
8.63 \\
9.41\end{array}$ & $\begin{array}{l}0.35 \\
0.24 \\
0.31\end{array}$ & $\begin{array}{l}2381 \\
2240 \\
2345 \\
\end{array}$ & $\begin{array}{l}85 \\
60 \\
67 \\
\end{array}$ & $\begin{array}{l}2218 \\
2224 \\
2104 \\
\end{array}$ & $\begin{array}{l}86 \\
75 \\
82 \\
\end{array}$ & $\begin{array}{l}416 \\
412 \\
416 \\
\end{array}$ & $\begin{array}{l}5 \\
6 \\
5 \\
\end{array}$ \\
\hline $\begin{array}{c}11944 \\
\text { Front } \\
\text { Center } \\
\text { Back }\end{array}$ & $\begin{array}{l}0.1004 \\
0.0885 \\
0.0963 \\
\end{array}$ & $\begin{array}{l}0.0036 \\
0.0032 \\
0.0032\end{array}$ & $\begin{array}{l}244.13 \\
251.07 \\
248.10\end{array}$ & $\begin{array}{l}4.25 \\
4.82 \\
4.25 \\
\end{array}$ & $\begin{array}{l}9.61 \\
9.88 \\
9.77\end{array}$ & $\begin{array}{l}0.17 \\
0.19 \\
0.17\end{array}$ & $\begin{array}{l}9.63 \\
8.78 \\
9.09 \\
\end{array}$ & $\begin{array}{l}0.38 \\
0.35 \\
0.35\end{array}$ & $\begin{array}{l}2281 \\
2282 \\
2277 \\
\end{array}$ & $\begin{array}{l}75 \\
64 \\
70 \\
\end{array}$ & $\begin{array}{l}2326 \\
2324 \\
2239 \\
\end{array}$ & $\begin{array}{l}108 \\
102 \\
99 \\
\end{array}$ & $\begin{array}{l}412 \\
416 \\
415 \\
\end{array}$ & $\begin{array}{l}5 \\
7 \\
4 \\
\end{array}$ \\
\hline $\begin{array}{l}\mathbf{1 1 9 4 5} \\
\text { Front } \\
\text { Center } \\
\text { Back }\end{array}$ & $\begin{array}{l}0.1067 \\
0.0900 \\
0.1005 \\
\end{array}$ & $\begin{array}{l}0.0045 \\
0.0032 \\
0.0043\end{array}$ & $\begin{array}{l}250.77 \\
254.87 \\
255.67\end{array}$ & $\begin{array}{l}6.14 \\
3.97 \\
5.32 \\
\end{array}$ & $\begin{array}{c}9.87 \\
10.03 \\
10.07 \\
\end{array}$ & $\begin{array}{l}0.24 \\
0.16 \\
0.21\end{array}$ & $\begin{array}{l}9.32 \\
8.53 \\
9.59 \\
\end{array}$ & $\begin{array}{l}0.28 \\
0.25 \\
0.30 \\
\end{array}$ & $\begin{array}{l}2343 \\
2282 \\
2368 \\
\end{array}$ & $\begin{array}{c}101 \\
99 \\
75 \\
\end{array}$ & $\begin{array}{l}2437 \\
2368 \\
2324 \\
\end{array}$ & $\begin{array}{l}119 \\
121 \\
110 \\
\end{array}$ & $\begin{array}{l}416 \\
415 \\
416 \\
\end{array}$ & $\begin{array}{l}5 \\
5 \\
5 \\
\end{array}$ \\
\hline $\begin{array}{c}11946 \\
\text { Front } \\
\text { Center } \\
\text { Back }\end{array}$ & $\begin{array}{l}0.0889 \\
0.0756 \\
0.0777\end{array}$ & $\begin{array}{l}0.0025 \\
0.0014 \\
0.0018\end{array}$ & $\begin{array}{l}244.65 \\
234.94 \\
235.30\end{array}$ & $\begin{array}{l}4.13 \\
1.86 \\
1.74\end{array}$ & $\begin{array}{l}9.63 \\
9.25 \\
9.26\end{array}$ & $\begin{array}{l}0.16 \\
0.07 \\
0.07\end{array}$ & $\begin{array}{c}10.71 \\
9.86 \\
12.24 \\
\end{array}$ & $\begin{array}{l}0.23 \\
0.30 \\
0.30 \\
\end{array}$ & $\begin{array}{l}1621 \\
1536 \\
1329 \\
\end{array}$ & $\begin{array}{c}91 \\
39 \\
150 \\
\end{array}$ & $\begin{array}{l}1604 \\
1487 \\
1238 \\
\end{array}$ & $\begin{array}{c}54 \\
38 \\
113 \\
\end{array}$ & $\begin{array}{l}378 \\
377 \\
350 \\
\end{array}$ & $\begin{array}{c}6 \\
3 \\
10 \\
\end{array}$ \\
\hline $\begin{array}{c}11947 \\
\text { Front } \\
\text { Center } \\
\text { Back }\end{array}$ & $\begin{array}{l}0.0889 \\
0.0752 \\
0.0859 \\
\end{array}$ & $\begin{array}{l}0.0033 \\
0.0022 \\
0.0029\end{array}$ & $\begin{array}{l}235.53 \\
240.10 \\
233.47\end{array}$ & $\begin{array}{l}2.58 \\
2.24 \\
2.43 \\
\end{array}$ & $\begin{array}{l}9.27 \\
9.45 \\
9.19 \\
\end{array}$ & $\begin{array}{l}0.10 \\
0.09 \\
0.10 \\
\end{array}$ & $\begin{array}{l}9.17 \\
8.39 \\
8.92 \\
\end{array}$ & $\begin{array}{l}0.25 \\
0.20 \\
0.27\end{array}$ & $\begin{array}{l}1908 \\
1647 \\
1704 \\
\end{array}$ & $\begin{array}{l}89 \\
73 \\
59 \\
\end{array}$ & $\begin{array}{l}1727 \\
1602 \\
1687 \\
\end{array}$ & $\begin{array}{l}70 \\
64 \\
76 \\
\end{array}$ & $\begin{array}{l}402 \\
395 \\
401 \\
\end{array}$ & $\begin{array}{l}5 \\
4 \\
5 \\
\end{array}$ \\
\hline $\begin{array}{c}11948 \\
\text { Front } \\
\text { Center } \\
\text { Back }\end{array}$ & $\begin{array}{l}0.0905 \\
0.0775 \\
0.0900\end{array}$ & $\begin{array}{l}0.0025 \\
0.0015 \\
0.0023\end{array}$ & $\begin{array}{l}238.45 \\
239.54 \\
239.30\end{array}$ & $\begin{array}{l}3.08 \\
1.73 \\
6.76\end{array}$ & $\begin{array}{l}9.39 \\
9.43 \\
9.42\end{array}$ & $\begin{array}{l}0.12 \\
0.07 \\
0.27\end{array}$ & $\begin{array}{c}10.60 \\
9.20 \\
10.14\end{array}$ & $\begin{array}{l}0.31 \\
0.21 \\
0.25\end{array}$ & $\begin{array}{l}1641 \\
1658 \\
1505 \\
\end{array}$ & $\begin{array}{l}82 \\
46 \\
64 \\
\end{array}$ & $\begin{array}{l}1669 \\
1646 \\
1508 \\
\end{array}$ & $\begin{array}{c}105 \\
42 \\
70 \\
\end{array}$ & $\begin{array}{l}379 \\
388 \\
377 \\
\end{array}$ & $\begin{array}{l}6 \\
3 \\
5 \\
\end{array}$ \\
\hline $\begin{array}{c}11949 \\
\text { Front } \\
\text { Center } \\
\text { Back }\end{array}$ & $\begin{array}{l}0.0873 \\
0.0771 \\
0.0895 \\
\end{array}$ & $\begin{array}{l}0.0025 \\
0.0015 \\
0.0021\end{array}$ & $\begin{array}{l}239.60 \\
239.89 \\
239.05 \\
\end{array}$ & $\begin{array}{l}5.32 \\
1.82 \\
4.73 \\
\end{array}$ & $\begin{array}{l}9.43 \\
9.44 \\
9.41 \\
\end{array}$ & $\begin{array}{l}0.21 \\
0.07 \\
0.19 \\
\end{array}$ & $\begin{array}{c}10.13 \\
9.10 \\
10.57 \\
\end{array}$ & $\begin{array}{l}0.38 \\
0.23 \\
0.22 \\
\end{array}$ & $\begin{array}{l}1479 \\
1722 \\
1593 \\
\end{array}$ & $\begin{array}{l}92 \\
38 \\
70 \\
\end{array}$ & $\begin{array}{l}1547 \\
1648 \\
1649 \\
\end{array}$ & $\begin{array}{c}109 \\
44 \\
73 \\
\end{array}$ & $\begin{array}{l}376 \\
389 \\
385 \\
\end{array}$ & $\begin{array}{l}6 \\
3 \\
4 \\
\end{array}$ \\
\hline $\begin{array}{c}1950 \\
\text { Front } \\
\text { Center } \\
\text { Back }\end{array}$ & $\begin{array}{l}0.1165 \\
0.1117 \\
0.1118\end{array}$ & $\begin{array}{l}0.0031 \\
0.0037 \\
0.0033\end{array}$ & $\begin{array}{l}224.90 \\
235.77 \\
229.97\end{array}$ & $\begin{array}{l}3.51 \\
4.65 \\
5.24\end{array}$ & $\begin{array}{l}8.85 \\
9.28 \\
9.05\end{array}$ & $\begin{array}{l}0.14 \\
0.18 \\
0.21\end{array}$ & $\begin{array}{l}16.26 \\
15.29 \\
14.71 \\
\end{array}$ & $\begin{array}{l}0.49 \\
0.43 \\
0.52\end{array}$ & $\begin{array}{l}1825 \\
1903 \\
1785\end{array}$ & $\begin{array}{l}120 \\
103 \\
107\end{array}$ & $\begin{array}{l}2296 \\
2379 \\
2277\end{array}$ & $\begin{array}{c}98 \\
116 \\
87\end{array}$ & $\begin{array}{l}396 \\
397 \\
396 \\
\end{array}$ & $\begin{array}{l}8 \\
7 \\
7 \\
\end{array}$ \\
\hline
\end{tabular}


Table E3 cont. Average Physical Property Data by Lane.

\begin{tabular}{|c|c|c|c|c|c|c|c|c|c|c|c|c|c|c|}
\hline Test Methods & IPST & & IPST & & & & $\mathrm{T} 460$ & & UM535 & & & & T538 & \\
\hline $\begin{array}{l}\text { Sample } \\
\text { ID } \\
(\#)\end{array}$ & $\begin{array}{l}\text { ZD-Long. } \\
\text { SEM } \\
\text { (MN m/kg) }\end{array}$ & $\begin{array}{l}95 \% \\
\text { C.I. }\end{array}$ & $\begin{array}{c}\text { Soft } \\
\text { Caliper } \\
\text { (microns) }\end{array}$ & $\begin{array}{c}95 \% \\
\text { C.I. }\end{array}$ & $\begin{array}{l}\text { Soft } \\
\text { Caliper } \\
\text { (mills) }\end{array}$ & $\begin{array}{c}95 \% \\
\text { C.I. }\end{array}$ & $\begin{array}{l}\text { Gurley } \\
\text { Porosity } \\
\text { (s/100ml) }\end{array}$ & $\begin{array}{c}95 \% \\
\text { C.I. }\end{array}$ & $\begin{array}{c}\text { FS } \\
\text { Bendtsen } \\
\text { Roughness } \\
\text { (ml/min) }\end{array}$ & $\begin{array}{c}95 \% \\
\text { C.I. }\end{array}$ & $\begin{array}{c}\text { WS } \\
\text { Bendtsen } \\
\text { Roughness } \\
(\mathrm{ml} / \mathrm{min})\end{array}$ & $\begin{array}{c}95 \% \\
\text { C.l. }\end{array}$ & $\begin{array}{c}\text { FS } \\
\text { Sheffield } \\
\text { Roughness } \\
\text { (SU) }\end{array}$ & $\begin{array}{c}95 \% \\
\text { C.I. }\end{array}$ \\
\hline $\begin{array}{c}11951 \\
\text { Front } \\
\text { Center } \\
\text { Back }\end{array}$ & $\begin{array}{l}0.1236 \\
0.1163 \\
0.1205\end{array}$ & $\begin{array}{l}0.0032 \\
0.0039 \\
0.0037\end{array}$ & $\begin{array}{l}219.07 \\
225.50 \\
217.27 \\
\end{array}$ & $\begin{array}{l}3.92 \\
4.58 \\
3.58\end{array}$ & $\begin{array}{l}8.62 \\
8.88 \\
8.55\end{array}$ & $\begin{array}{l}0.15 \\
0.18 \\
0.14\end{array}$ & $\begin{array}{l}15.59 \\
15.16 \\
14.95 \\
\end{array}$ & $\begin{array}{l}0.20 \\
0.33 \\
0.27\end{array}$ & $\begin{array}{l}1916 \\
1886 \\
1838 \\
\end{array}$ & $\begin{array}{l}130 \\
119 \\
108 \\
\end{array}$ & $\begin{array}{l}2194 \\
2437 \\
2328 \\
\end{array}$ & $\begin{array}{c}94 \\
106 \\
95 \\
\end{array}$ & $\begin{array}{l}397 \\
400 \\
395 \\
\end{array}$ & $\begin{array}{c}11 \\
8 \\
10 \\
\end{array}$ \\
\hline $\begin{array}{l}11952 \\
\text { Front } \\
\text { Center } \\
\text { Back }\end{array}$ & $\begin{array}{l}0.1300 \\
0.1220 \\
0.1245\end{array}$ & $\begin{array}{l}0.0032 \\
0.0031 \\
0.0037\end{array}$ & $\begin{array}{l}212.28 \\
216.40 \\
217.10\end{array}$ & $\begin{array}{l}3.84 \\
3.51 \\
4.88\end{array}$ & $\begin{array}{l}8.36 \\
8.52 \\
8.55\end{array}$ & $\begin{array}{l}0.15 \\
0.14 \\
0.19\end{array}$ & $\begin{array}{l}17.30 \\
16.20 \\
16.63\end{array}$ & $\begin{array}{l}0.53 \\
0.42 \\
0.49\end{array}$ & $\begin{array}{l}1868 \\
1734 \\
1817\end{array}$ & $\begin{array}{l}96 \\
80 \\
95\end{array}$ & $\begin{array}{l}2367 \\
2347 \\
2436\end{array}$ & $\begin{array}{c}107 \\
81 \\
83\end{array}$ & $\begin{array}{l}399 \\
396 \\
390\end{array}$ & $\begin{array}{l}8 \\
6 \\
8\end{array}$ \\
\hline $\begin{array}{l}11953 \\
\text { Front } \\
\text { Center } \\
\text { Back }\end{array}$ & $\begin{array}{l}0.1307 \\
0.1206 \\
0.1246\end{array}$ & $\begin{array}{l}0.0033 \\
0.0031 \\
0.0033\end{array}$ & $\begin{array}{l}215.20 \\
220.30 \\
218.88\end{array}$ & $\begin{array}{l}4.47 \\
4.07 \\
5.36\end{array}$ & $\begin{array}{l}8.47 \\
8.67 \\
8.62\end{array}$ & $\begin{array}{l}0.18 \\
0.16 \\
0.21\end{array}$ & $\begin{array}{l}19.55 \\
16.84 \\
18.30\end{array}$ & $\begin{array}{l}0.61 \\
0.40 \\
0.56\end{array}$ & $\begin{array}{l}1808 \\
1746 \\
1693\end{array}$ & $\begin{array}{l}116 \\
106 \\
94\end{array}$ & $\begin{array}{l}2358 \\
2405 \\
2238\end{array}$ & $\begin{array}{l}98 \\
94 \\
89\end{array}$ & $\begin{array}{l}403 \\
405 \\
396\end{array}$ & $\begin{array}{l}9 \\
8 \\
7\end{array}$ \\
\hline $\begin{array}{l}11954 \\
\text { Front } \\
\text { Center } \\
\text { Back }\end{array}$ & $\begin{array}{l}0.1327 \\
0.1235 \\
0.1311\end{array}$ & $\begin{array}{l}0.0031 \\
0.0035 \\
0.0036\end{array}$ & $\begin{array}{l}219.18 \\
221.15 \\
223.33\end{array}$ & $\begin{array}{l}4.73 \\
3.86 \\
4.29\end{array}$ & $\begin{array}{l}8.64 \\
8.71 \\
8.79\end{array}$ & $\begin{array}{l}0.19 \\
0.15 \\
0.17\end{array}$ & $\begin{array}{l}19.15 \\
16.12 \\
18.30\end{array}$ & $\begin{array}{l}0.46 \\
0.41 \\
0.54\end{array}$ & $\begin{array}{l}1853 \\
1758 \\
1944\end{array}$ & $\begin{array}{c}113 \\
93 \\
111\end{array}$ & $\begin{array}{l}2303 \\
2385 \\
2280\end{array}$ & $\begin{array}{c}73 \\
103 \\
85\end{array}$ & $\begin{array}{l}398 \\
401 \\
407\end{array}$ & $\begin{array}{c}6 \\
10 \\
7\end{array}$ \\
\hline $\begin{array}{l}11955 \\
\text { Front } \\
\text { Center } \\
\text { Back }\end{array}$ & $\begin{array}{l}0.1233 \\
0.1065 \\
0.1188\end{array}$ & $\begin{array}{l}0.0041 \\
0.0026 \\
0.0047\end{array}$ & $\begin{array}{l}226.07 \\
238.30 \\
235.00\end{array}$ & $\begin{array}{l}5.81 \\
6.40 \\
6.02\end{array}$ & $\begin{array}{l}8.90 \\
9.38 \\
9.25\end{array}$ & $\begin{array}{l}0.23 \\
0.25 \\
0.24\end{array}$ & $\begin{array}{l}17.17 \\
14.39 \\
16.62\end{array}$ & $\begin{array}{l}0.61 \\
0.27 \\
0.54\end{array}$ & $\begin{array}{l}1961 \\
1859 \\
1916\end{array}$ & $\begin{array}{l}150 \\
130 \\
113\end{array}$ & $\begin{array}{l}2284 \\
2426 \\
2269\end{array}$ & $\begin{array}{c}92 \\
135 \\
93\end{array}$ & $\begin{array}{l}407 \\
398 \\
397\end{array}$ & $\begin{array}{c}8 \\
11 \\
10\end{array}$ \\
\hline $\begin{array}{c}11956 \\
\text { Front } \\
\text { Center } \\
\text { Back }\end{array}$ & $\begin{array}{l}0.1287 \\
0.1140 \\
0.1235\end{array}$ & $\begin{array}{l}0.0054 \\
0.0029 \\
0.0046\end{array}$ & $\begin{array}{l}220.10 \\
229.43 \\
228.17\end{array}$ & $\begin{array}{l}5.03 \\
7.15 \\
5.79\end{array}$ & $\begin{array}{l}8.67 \\
9.03 \\
8.98\end{array}$ & $\begin{array}{l}0.20 \\
0.28 \\
0.23\end{array}$ & $\begin{array}{l}19.27 \\
15.86 \\
18.54\end{array}$ & $\begin{array}{l}0.75 \\
0.28 \\
0.53\end{array}$ & $\begin{array}{l}1872 \\
1876 \\
1982\end{array}$ & $\begin{array}{l}146 \\
129 \\
144\end{array}$ & $\begin{array}{l}2267 \\
2282 \\
2214\end{array}$ & $\begin{array}{c}92 \\
108 \\
103\end{array}$ & $\begin{array}{l}404 \\
393 \\
408\end{array}$ & $\begin{array}{c}9 \\
11 \\
6\end{array}$ \\
\hline
\end{tabular}


Table E3 cont. Average Physical Property Data by Lane.

\begin{tabular}{|c|c|c|c|c|c|c|c|c|c|c|c|c|c|c|}
\hline Test Methods & IPST & & IPST & & & & $\mathrm{T} 460$ & & UM535 & & & & T538 & \\
\hline $\begin{array}{c}\text { Sample } \\
\text { ID } \\
(\#)\end{array}$ & $\begin{array}{l}\text { ZD-Long. } \\
\text { SEM } \\
\text { (MN m/kg) }\end{array}$ & $\begin{array}{l}95 \% \\
\text { C.I. }\end{array}$ & $\begin{array}{c}\text { Soft } \\
\text { Caliper } \\
\text { (microns) }\end{array}$ & $\begin{array}{l}95 \% \\
\text { C.I. }\end{array}$ & $\begin{array}{c}\text { Soft } \\
\text { Caliper } \\
\text { (mills) }\end{array}$ & $\begin{array}{l}95 \% \\
\text { C.I. }\end{array}$ & $\begin{array}{c}\text { Gurley } \\
\text { Porosity } \\
\text { (s/100ml) }\end{array}$ & $\begin{array}{l}95 \% \\
\text { C.I. }\end{array}$ & $\begin{array}{c}\text { FS } \\
\text { Bendtsen } \\
\text { Roughness } \\
\text { (ml/min) } \\
\end{array}$ & $\begin{array}{c}95 \% \\
\text { C.I. }\end{array}$ & $\begin{array}{c}\text { WS } \\
\text { Bendtsen } \\
\text { Roughness } \\
\text { (ml/min) } \\
\end{array}$ & $\begin{array}{l}95 \% \\
\text { C.I. }\end{array}$ & $\begin{array}{c}\text { FS } \\
\text { Sheffield } \\
\text { Roughness } \\
\text { (SU) } \\
\end{array}$ & $\begin{array}{c}95 \% \\
\text { C.I. }\end{array}$ \\
\hline $\begin{array}{c}\text { Commercial } \\
\text { Medium } \\
\mathbf{1 0 7 8 1 1} \\
\text { Center } \\
\text { Edge }\end{array}$ & $\begin{array}{l}0.1397 \\
0.1387\end{array}$ & $\begin{array}{l}0.0026 \\
0.0019\end{array}$ & $\begin{array}{l}187.65 \\
190.08\end{array}$ & $\begin{array}{l}2.61 \\
2.59\end{array}$ & $\begin{array}{l}7.39 \\
7.48 \\
\end{array}$ & $\begin{array}{l}0.10 \\
0.10\end{array}$ & $\begin{array}{l}10.32 \\
10.97\end{array}$ & $\begin{array}{l}0.46 \\
0.40\end{array}$ & $\begin{array}{l}2792 \\
2792 \\
\end{array}$ & $\begin{array}{l}97 \\
76 \\
\end{array}$ & $\begin{array}{l}2591 \\
2588 \\
\end{array}$ & $\begin{array}{c}145 \\
84 \\
\end{array}$ & & \\
\hline $\begin{array}{l}\mathbf{1 0 7 8 1 2} \\
\text { Center } \\
\text { Edge }\end{array}$ & $\begin{array}{l}0.1347 \\
0.1342\end{array}$ & $\begin{array}{l}0.0034 \\
0.0024\end{array}$ & $\begin{array}{l}180.95 \\
182.90\end{array}$ & $\begin{array}{l}2.90 \\
2.36\end{array}$ & $\begin{array}{l}7.12 \\
7.20\end{array}$ & $\begin{array}{l}0.11 \\
0.09\end{array}$ & $\begin{array}{l}11.76 \\
11.05\end{array}$ & $\begin{array}{l}0.74 \\
0.31\end{array}$ & $\begin{array}{l}2520 \\
2651\end{array}$ & $\begin{array}{c}119 \\
83\end{array}$ & $\begin{array}{l}2681 \\
2905 \\
\end{array}$ & $\begin{array}{l}89 \\
85 \\
\end{array}$ & & \\
\hline $\begin{array}{c}107813 \\
\text { Center } \\
\text { Edge }\end{array}$ & $\begin{array}{l}0.1500 \\
0.1486\end{array}$ & $\begin{array}{l}0.0029 \\
0.0024\end{array}$ & $\begin{array}{l}180.35 \\
180.05\end{array}$ & $\begin{array}{l}3.54 \\
2.08\end{array}$ & $\begin{array}{l}7.10 \\
7.09 \\
\end{array}$ & $\begin{array}{l}0.14 \\
0.08\end{array}$ & $\begin{array}{l}11.10 \\
11.80\end{array}$ & $\begin{array}{l}0.48 \\
0.37 \\
\end{array}$ & $\begin{array}{l}3024 \\
3041 \\
\end{array}$ & $\begin{array}{l}96 \\
63 \\
\end{array}$ & $\begin{array}{l}2815 \\
2778 \\
\end{array}$ & $\begin{array}{l}81 \\
77 \\
\end{array}$ & & \\
\hline $\begin{array}{c}107814 \\
\text { Center } \\
\text { Edge }\end{array}$ & $\begin{array}{l}0.1400 \\
0.1385\end{array}$ & $\begin{array}{l}0.0027 \\
0.0024\end{array}$ & $\begin{array}{l}188.95 \\
190.15\end{array}$ & $\begin{array}{l}3.39 \\
2.39\end{array}$ & $\begin{array}{l}7.44 \\
7.49 \\
\end{array}$ & $\begin{array}{l}0.13 \\
0.09\end{array}$ & $\begin{array}{l}10.50 \\
10.80\end{array}$ & $\begin{array}{l}0.52 \\
0.41\end{array}$ & $\begin{array}{l}2905 \\
2915 \\
\end{array}$ & $\begin{array}{l}86 \\
51 \\
\end{array}$ & $\begin{array}{l}2650 \\
2701 \\
\end{array}$ & $\begin{array}{c}109 \\
99 \\
\end{array}$ & & \\
\hline $\begin{array}{c}107816 \\
\text { Center } \\
\text { Edge } \\
\end{array}$ & $\begin{array}{l}0.1487 \\
0.1492 \\
\end{array}$ & $\begin{array}{l}0.0031 \\
0.0024 \\
\end{array}$ & $\begin{array}{l}183.20 \\
186.10 \\
\end{array}$ & $\begin{array}{l}2.98 \\
2.17 \\
\end{array}$ & $\begin{array}{l}7.21 \\
7.33 \\
\end{array}$ & $\begin{array}{l}0.12 \\
0.09 \\
\end{array}$ & $\begin{array}{l}11.83 \\
11.85 \\
\end{array}$ & $\begin{array}{l}0.53 \\
0.34 \\
\end{array}$ & $\begin{array}{l}2792 \\
2800 \\
\end{array}$ & $\begin{array}{r}153 \\
84 \\
\end{array}$ & $\begin{array}{l}2942 \\
2885 \\
\end{array}$ & $\begin{array}{r}104 \\
84 \\
\end{array}$ & & \\
\hline $\begin{array}{c}\text { Commercial } \\
\text { Liner } \\
225111 \\
\text { Center } \\
\text { Edge } \\
\end{array}$ & $\begin{array}{l}0.1194 \\
0.1245\end{array}$ & $\begin{array}{l}0.0066 \\
0.0033\end{array}$ & $\begin{array}{l}209.25 \\
208.58\end{array}$ & $\begin{array}{l}5.34 \\
1.89\end{array}$ & $\begin{array}{l}8.24 \\
8.21\end{array}$ & $\begin{array}{l}0.21 \\
0.07\end{array}$ & $\begin{array}{l}15.63 \\
15.57\end{array}$ & $\begin{array}{l}0.59 \\
0.50\end{array}$ & $\begin{array}{l}1933 \\
2092\end{array}$ & $\begin{array}{l}167 \\
124 \\
\end{array}$ & $\begin{array}{l}1817 \\
2211 \\
\end{array}$ & $\begin{array}{l}128 \\
168\end{array}$ & $\begin{array}{l}394 \\
393\end{array}$ & $\begin{array}{l}8 \\
5 \\
\end{array}$ \\
\hline $\begin{array}{c}225121 \\
\text { Center } \\
\text { Edge }\end{array}$ & $\begin{array}{l}0.1034 \\
0.1118\end{array}$ & $\begin{array}{l}0.0053 \\
0.0039\end{array}$ & $\begin{array}{l}214.10 \\
209.23\end{array}$ & $\begin{array}{l}3.56 \\
3.40\end{array}$ & $\begin{array}{l}8.43 \\
8.24\end{array}$ & $\begin{array}{l}0.14 \\
0.13\end{array}$ & $\begin{array}{l}17.67 \\
16.31\end{array}$ & $\begin{array}{l}0.75 \\
0.61\end{array}$ & $\begin{array}{l}2182 \\
2327 \\
\end{array}$ & $\begin{array}{l}267 \\
163 \\
\end{array}$ & $\begin{array}{l}2004 \\
2195 \\
\end{array}$ & $\begin{array}{l}230 \\
147 \\
\end{array}$ & $\begin{array}{r}389 \\
396 \\
\end{array}$ & $\begin{array}{c}10 \\
6 \\
\end{array}$ \\
\hline
\end{tabular}


Table E3 cont. Average Physical Property Data by Lane.

\begin{tabular}{|c|c|c|c|c|c|c|c|c|c|c|c|c|c|c|}
\hline Test Methods & & & & & & & IPST & & & & & & & \\
\hline $\begin{array}{c}\text { Sample } \\
\text { ID } \\
(\#)\end{array}$ & $\begin{array}{c}\text { WS } \\
\text { Sheffield } \\
\text { Roughness } \\
\text { (SU) }\end{array}$ & $\begin{array}{l}95 \% \\
\text { C.1. }\end{array}$ & $\begin{array}{c}\text { FS } \\
\text { Sheffield } \\
\text { Roughness } \\
(\mathrm{m} / \mathrm{min})\end{array}$ & $\begin{array}{l}95 \% \\
\text { C.I. }\end{array}$ & $\begin{array}{c}\text { WS } \\
\text { Sheffield } \\
\text { Roughness } \\
(\mathrm{ml} / \mathrm{min})\end{array}$ & $\begin{array}{l}95 \% \\
\text { C.I. }\end{array}$ & $\begin{array}{c}\text { CD } \\
\text { FS Emveco } \\
\text { Micro Dev. }\end{array}$ & $\begin{array}{c}95 \% \\
\text { C.1. }\end{array}$ & $\begin{array}{c}c \text { CD } \\
\text { FS Emveco } \\
\text { Micro Ave. }\end{array}$ & $\begin{array}{l}95 \% \\
\text { C.I. }\end{array}$ & $\begin{array}{c}\text { MD } \\
\text { FS Emveco } \\
\text { Micro Dev. }\end{array}$ & $\begin{array}{l}95 \% \\
\text { C.I. }\end{array}$ & $\begin{array}{c}\text { MD } \\
\text { FS Emveco } \\
\text { Micro Ave. }\end{array}$ & $\begin{array}{l}95 \% \\
\text { C.I. }\end{array}$ \\
\hline $\begin{array}{c}11926 \\
\text { Front } \\
\text { Center } \\
\text { Back }\end{array}$ & $\begin{array}{l}421 \\
420 \\
418 \\
\end{array}$ & $\begin{array}{l}7 \\
5 \\
5\end{array}$ & $\begin{array}{l}3365 \\
3381 \\
3373\end{array}$ & $\begin{array}{l}55 \\
76 \\
57\end{array}$ & $\begin{array}{l}3524 \\
3515 \\
3498\end{array}$ & $\begin{array}{l}59 \\
40 \\
38\end{array}$ & $\begin{array}{l}80.9 \\
82.4 \\
88.4\end{array}$ & $\begin{array}{l}7.6 \\
5.3 \\
7.1 \\
\end{array}$ & $\begin{array}{l}0.2246 \\
0.2286 \\
0.2348\end{array}$ & $\begin{array}{l}0.0102 \\
0.0075 \\
0.0066\end{array}$ & $\begin{array}{l}51.2 \\
58.7 \\
57.0\end{array}$ & $\begin{array}{l}8.6 \\
8.9 \\
8.3\end{array}$ & $\begin{array}{l}0.1764 \\
0.1904 \\
0.1874\end{array}$ & $\begin{array}{l}0.0109 \\
0.0130 \\
0.0104\end{array}$ \\
\hline $\begin{array}{c}\mathbf{1 1 9 2 7} \\
\text { Front } \\
\text { Center } \\
\text { Back } \\
\end{array}$ & $\begin{array}{l}401 \\
384 \\
376 \\
\end{array}$ & $\begin{array}{l}8 \\
6 \\
6 \\
\end{array}$ & $\begin{array}{l}3147 \\
2930 \\
3055 \\
\end{array}$ & $\begin{array}{l}94 \\
80 \\
81 \\
\end{array}$ & $\begin{array}{l}3356 \\
3214 \\
3147 \\
\end{array}$ & $\begin{array}{l}66 \\
50 \\
50 \\
\end{array}$ & $\begin{array}{l}73.6 \\
66.7 \\
66.2 \\
\end{array}$ & $\begin{array}{l}5.5 \\
4.0 \\
3.8 \\
\end{array}$ & $\begin{array}{l}0.2142 \\
0.2034 \\
0.2023 \\
\end{array}$ & $\begin{array}{l}0.0092 \\
0.0086 \\
0.0065 \\
\end{array}$ & $\begin{array}{l}49.4 \\
47.0 \\
45.8 \\
\end{array}$ & $\begin{array}{c}10.4 \\
10.2 \\
7.6 \\
\end{array}$ & $\begin{array}{l}0.1729 \\
0.1684 \\
0.1655 \\
\end{array}$ & $\begin{array}{l}0.0171 \\
0.0162 \\
0.0128 \\
\end{array}$ \\
\hline $\begin{array}{c}\mathbf{1 1 9 2 8} \\
\text { Front } \\
\text { Center } \\
\text { Back }\end{array}$ & $\begin{array}{l}398 \\
383 \\
379\end{array}$ & $\begin{array}{l}5 \\
6 \\
5\end{array}$ & $\begin{array}{l}3164 \\
2930 \\
2980\end{array}$ & $\begin{array}{l}62 \\
89 \\
70\end{array}$ & $\begin{array}{l}3331 \\
3206 \\
3172\end{array}$ & $\begin{array}{l}38 \\
49 \\
44\end{array}$ & $\begin{array}{l}72.0 \\
69.5 \\
68.7\end{array}$ & $\begin{array}{l}5.0 \\
4.5 \\
1.8\end{array}$ & $\begin{array}{l}0.2097 \\
0.2071 \\
0.2067\end{array}$ & $\begin{array}{l}0.0096 \\
0.0062 \\
0.0016\end{array}$ & $\begin{array}{l}47.9 \\
46.6 \\
49.4\end{array}$ & $\begin{array}{l}2.4 \\
5.4 \\
7.8\end{array}$ & $\begin{array}{l}0.1711 \\
0.1696 \\
0.1731\end{array}$ & $\begin{array}{l}0.0056 \\
0.0093 \\
0.0149\end{array}$ \\
\hline $\begin{array}{c}11930 \\
\text { Front } \\
\text { Center } \\
\text { Back } \\
\end{array}$ & $\begin{array}{l}417 \\
418 \\
417 \\
\end{array}$ & $\begin{array}{l}5 \\
5 \\
5 \\
\end{array}$ & $\begin{array}{l}3281 \\
3239 \\
3298 \\
\end{array}$ & $\begin{array}{l}62 \\
57 \\
50 \\
\end{array}$ & $\begin{array}{l}3490 \\
3498 \\
3490 \\
\end{array}$ & $\begin{array}{l}40 \\
38 \\
40 \\
\end{array}$ & $\begin{array}{l}85.7 \\
87.2 \\
93.0 \\
\end{array}$ & $\begin{array}{r}6.7 \\
6.2 \\
20.2 \\
\end{array}$ & $\begin{array}{l}0.2301 \\
0.2332 \\
0.2362 \\
\end{array}$ & $\begin{array}{l}0.0103 \\
0.0105 \\
0.0146 \\
\end{array}$ & $\begin{array}{l}53.3 \\
58.0 \\
53.5 \\
\end{array}$ & $\begin{array}{l}6.7 \\
3.8 \\
4.7 \\
\end{array}$ & $\begin{array}{l}0.1788 \\
0.1905 \\
0.1818 \\
\end{array}$ & $\begin{array}{l}0.0089 \\
0.0062 \\
0.0072 \\
\end{array}$ \\
\hline $\begin{array}{c}11931 \\
\text { Front } \\
\text { Center } \\
\text { Back }\end{array}$ & $\begin{array}{l}412 \\
420 \\
419 \\
\end{array}$ & $\begin{array}{l}6 \\
5 \\
6\end{array}$ & $\begin{array}{l}3373 \\
3315 \\
3331 \\
\end{array}$ & $\begin{array}{l}69 \\
42 \\
55\end{array}$ & $\begin{array}{l}3448 \\
3515 \\
3507\end{array}$ & $\begin{array}{l}47 \\
40 \\
52\end{array}$ & $\begin{array}{l}77.6 \\
82.5 \\
89.6 \\
\end{array}$ & $\begin{array}{l}2.5 \\
4.7 \\
3.0\end{array}$ & $\begin{array}{l}0.2213 \\
0.2275 \\
0.2375\end{array}$ & $\begin{array}{l}0.0045 \\
0.0037 \\
0.0046\end{array}$ & $\begin{array}{l}52.1 \\
55.5 \\
54.8 \\
\end{array}$ & $\begin{array}{l}3.5 \\
4.8 \\
8.2 \\
\end{array}$ & $\begin{array}{l}0.1823 \\
0.1861 \\
0.1809\end{array}$ & $\begin{array}{l}0.0081 \\
0.0106 \\
0.0120\end{array}$ \\
\hline $\begin{array}{l}11932 \\
\text { Front } \\
\text { Center } \\
\text { Back }\end{array}$ & $\begin{array}{l}409 \\
406 \\
409\end{array}$ & $\begin{array}{l}7 \\
6 \\
4\end{array}$ & $\begin{array}{l}3348 \\
3365 \\
3340\end{array}$ & $\begin{array}{l}63 \\
55 \\
52\end{array}$ & $\begin{array}{l}3423 \\
3398 \\
3423\end{array}$ & $\begin{array}{l}59 \\
50 \\
34\end{array}$ & $\begin{array}{l}79.8 \\
85.7 \\
87.9\end{array}$ & $\begin{array}{l}3.7 \\
7.6 \\
8.2\end{array}$ & $\begin{array}{l}0.2242 \\
0.2305 \\
0.2331\end{array}$ & $\begin{array}{l}0.0051 \\
0.0118 \\
0.0107\end{array}$ & $\begin{array}{l}54.4 \\
56.9 \\
53.4\end{array}$ & $\begin{array}{l}7.2 \\
4.6 \\
8.8\end{array}$ & $\begin{array}{l}0.1846 \\
0.1876 \\
0.1783\end{array}$ & $\begin{array}{l}0.0145 \\
0.0054 \\
0.0187\end{array}$ \\
\hline $\begin{array}{l}11933 \\
\text { Front } \\
\text { Center } \\
\text { Back }\end{array}$ & $\begin{array}{l}404 \\
412 \\
407\end{array}$ & $\begin{array}{l}6 \\
7 \\
5\end{array}$ & $\begin{array}{l}3340 \\
3298 \\
3340\end{array}$ & $\begin{array}{l}66 \\
58 \\
59\end{array}$ & $\begin{array}{l}3381 \\
3448 \\
3407\end{array}$ & $\begin{array}{l}50 \\
62 \\
40\end{array}$ & $\begin{array}{l}74.2 \\
81.5 \\
84.0 \\
\end{array}$ & $\begin{array}{l}5.3 \\
4.1 \\
5.2\end{array}$ & $\begin{array}{l}0.2150 \\
0.2260 \\
0.2286\end{array}$ & $\begin{array}{l}0.0088 \\
0.0057 \\
0.0067\end{array}$ & $\begin{array}{l}51.0 \\
53.3 \\
52.4 \\
\end{array}$ & $\begin{array}{l}4.2 \\
4.3 \\
3.9\end{array}$ & $\begin{array}{l}0.1741 \\
0.1792 \\
0.1781\end{array}$ & $\begin{array}{l}0.0082 \\
0.0071 \\
0.0027\end{array}$ \\
\hline $\begin{array}{l}11934 \\
\text { Front } \\
\text { Center } \\
\text { Back }\end{array}$ & $\begin{array}{l}411 \\
417 \\
407\end{array}$ & $\begin{array}{l}4 \\
7 \\
7\end{array}$ & $\begin{array}{l}3356 \\
3331 \\
3323\end{array}$ & $\begin{array}{l}77 \\
62 \\
80\end{array}$ & $\begin{array}{l}3440 \\
3490 \\
3407\end{array}$ & $\begin{array}{l}34 \\
57 \\
57\end{array}$ & $\begin{array}{l}78.5 \\
84.5 \\
84.2\end{array}$ & $\begin{array}{c}4.1 \\
5.6 \\
11.2\end{array}$ & $\begin{array}{l}0.2207 \\
0.2279 \\
0.2295\end{array}$ & $\begin{array}{l}0.0037 \\
0.0071 \\
0.0137\end{array}$ & $\begin{array}{l}52.2 \\
52.2 \\
52.6\end{array}$ & $\begin{array}{c}5.5 \\
13.6 \\
5.2\end{array}$ & $\begin{array}{l}0.1768 \\
0.1793 \\
0.1807\end{array}$ & $\begin{array}{l}0.0084 \\
0.0185 \\
0.0139\end{array}$ \\
\hline
\end{tabular}


Table E3 cont. Average Physical Property Data by Lane.

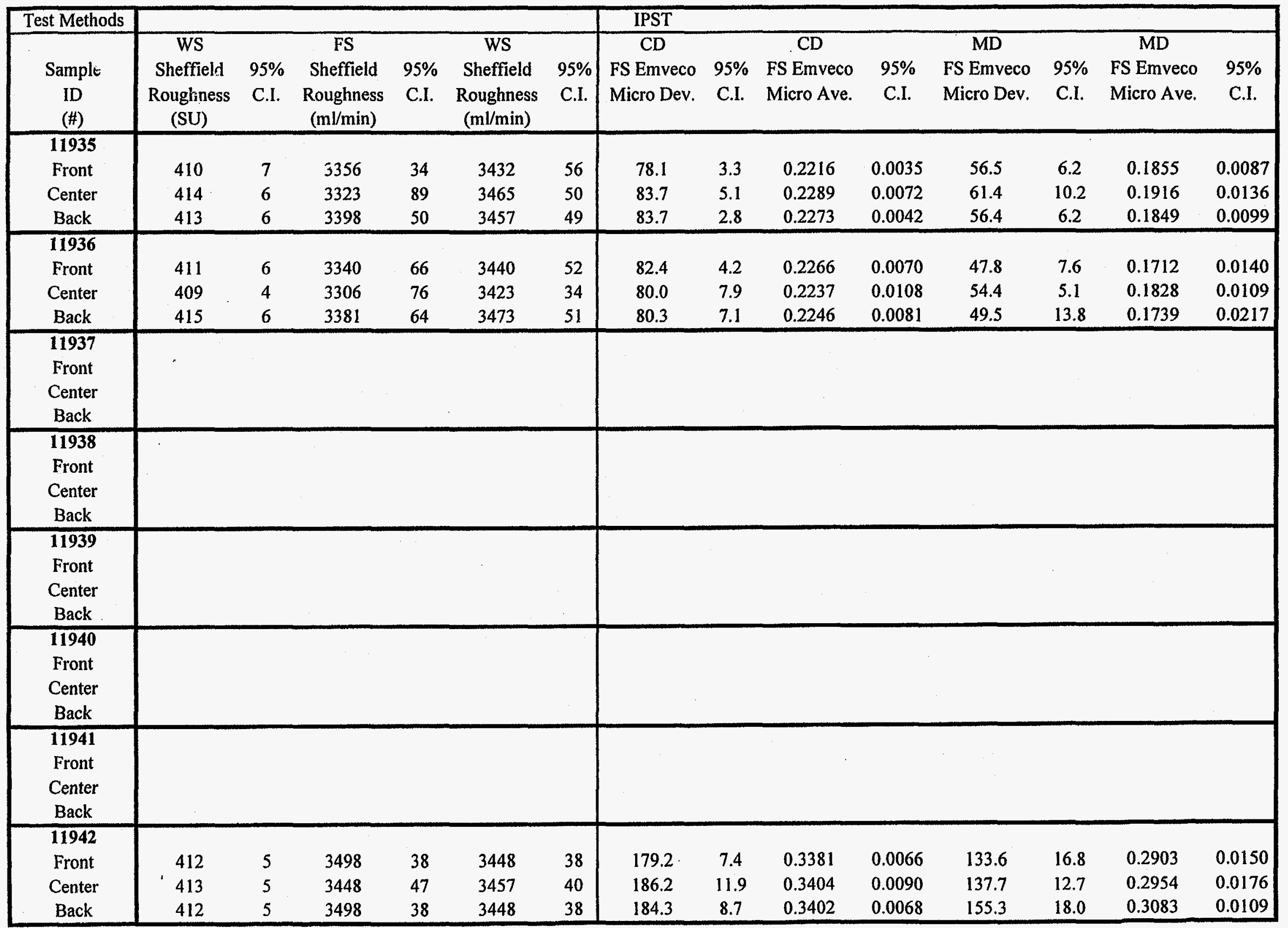


Table E3 cont. Average Physical Property Data by Lane.

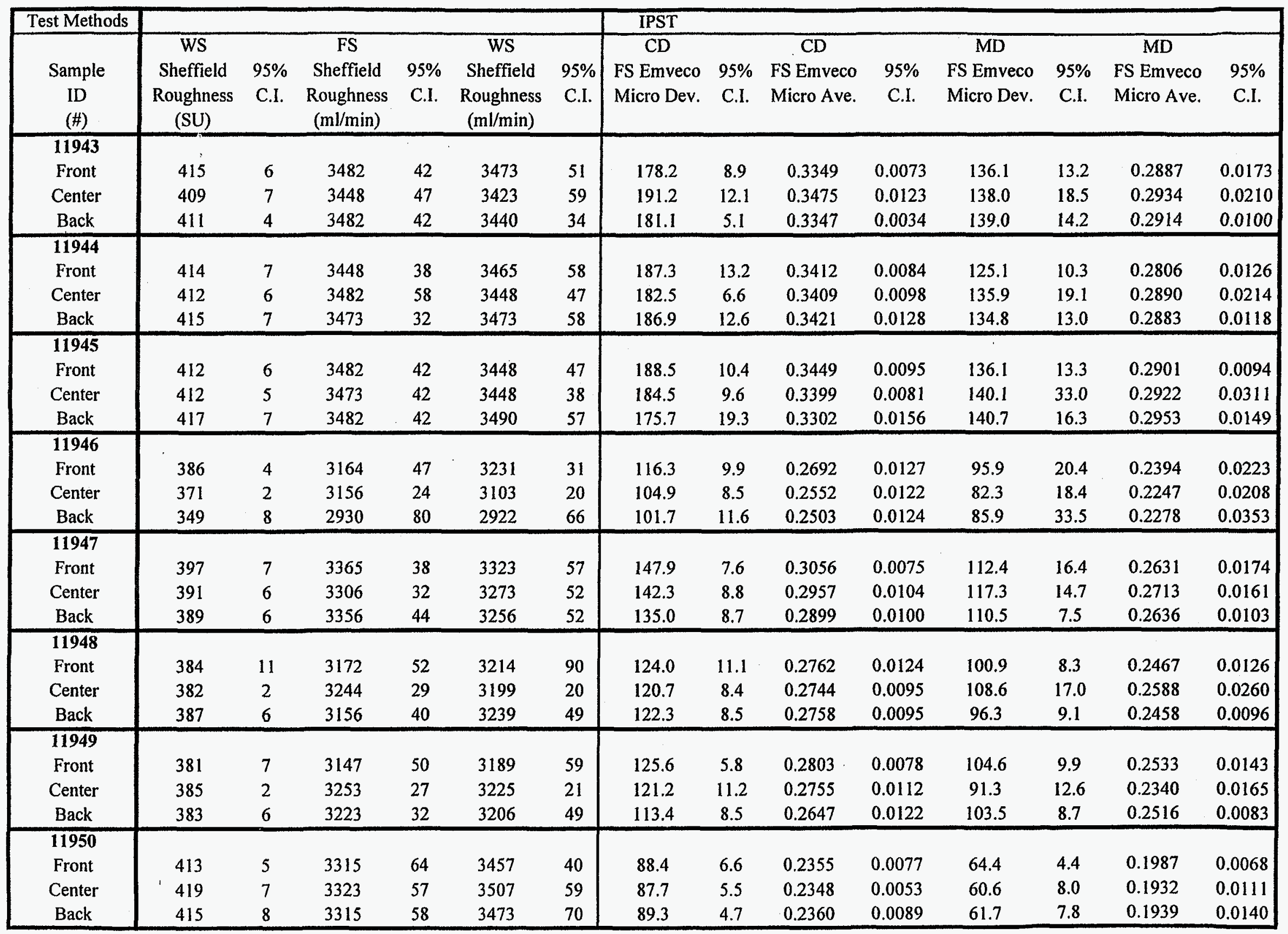


Table E3 cont. Average Physical Property Data by Lane.

\begin{tabular}{|c|c|c|c|c|c|c|c|c|c|c|c|c|c|c|}
\hline Test Methods & & & & & & & IPST & & & & & & & \\
\hline $\begin{array}{c}\text { Sample } \\
\text { ID } \\
(\#)\end{array}$ & $\begin{array}{c}\text { WS } \\
\text { Sheffield } \\
\text { Roughness } \\
\text { (SU) }\end{array}$ & $\begin{array}{l}95 \% \\
\text { C.I. }\end{array}$ & $\begin{array}{c}\text { FS } \\
\text { Sheffield } \\
\text { Roughness } \\
\text { (ml/min) }\end{array}$ & $\begin{array}{l}95 \% \\
\text { C.I. }\end{array}$ & $\begin{array}{c}\text { WS } \\
\text { Sheffield } \\
\text { Roughness } \\
(\mathrm{ml} / \mathrm{min})\end{array}$ & $\begin{array}{c}95 \% \\
\text { C.I. }\end{array}$ & $\begin{array}{c}\mathrm{CD} \\
\text { FS Emveco } \\
\text { Micro Dev. }\end{array}$ & $\begin{array}{c}95 \% \\
\text { C.I. }\end{array}$ & $\begin{array}{c}c \text { CD } \\
\text { FS Emveco } \\
\text { Micro Ave. }\end{array}$ & $\begin{array}{l}95 \% \\
\text { C.I. }\end{array}$ & $\begin{array}{c}\text { MD } \\
\text { FS Emveco } \\
\text { Micro Dev. }\end{array}$ & $\begin{array}{l}95 \% \\
\text { C.I. }\end{array}$ & $\begin{array}{c}\text { MD } \\
\text { FS Emveco } \\
\text { Micro Ave. }\end{array}$ & $\begin{array}{l}95 \% \\
\text { C.I. }\end{array}$ \\
\hline $\begin{array}{c}11951 \\
\text { Front } \\
\text { Center } \\
\text { Back }\end{array}$ & $\begin{array}{l}411 \\
410 \\
408\end{array}$ & $\begin{array}{l}2 \\
8 \\
6\end{array}$ & $\begin{array}{l}3323 \\
3348 \\
3306\end{array}$ & $\begin{array}{l}89 \\
69 \\
86\end{array}$ & $\begin{array}{l}3440 \\
3432 \\
3415\end{array}$ & $\begin{array}{l}19 \\
63 \\
47\end{array}$ & $\begin{array}{l}83.1 \\
82.8 \\
82.4\end{array}$ & $\begin{array}{c}7.2 \\
10.2 \\
6.4\end{array}$ & $\begin{array}{l}0.2268 \\
0.2259 \\
0.2261\end{array}$ & $\begin{array}{l}0.0101 \\
0.0141 \\
0.0079 \\
\end{array}$ & $\begin{array}{l}57.3 \\
59.9 \\
53.3\end{array}$ & $\begin{array}{c}14.6 \\
8.4 \\
4.0\end{array}$ & $\begin{array}{l}0.1887 \\
0.1939 \\
0.1819 \\
\end{array}$ & $\begin{array}{l}0.0253 \\
0.0173 \\
0.0096\end{array}$ \\
\hline $\begin{array}{l}11952 \\
\text { Front } \\
\text { Center } \\
\text { Back }\end{array}$ & $\begin{array}{l}416 \\
415 \\
413\end{array}$ & $\begin{array}{l}6 \\
6 \\
6\end{array}$ & $\begin{array}{l}3340 \\
3315 \\
3264\end{array}$ & $\begin{array}{l}66 \\
50 \\
63\end{array}$ & $\begin{array}{l}3482 \\
3473 \\
3457\end{array}$ & $\begin{array}{l}50 \\
51 \\
49\end{array}$ & $\begin{array}{l}78.8 \\
79.0 \\
78.5\end{array}$ & $\begin{array}{l}3.6 \\
2.4 \\
4.0\end{array}$ & $\begin{array}{l}0.2204 \\
0.2221 \\
0.2221\end{array}$ & $\begin{array}{l}0.0047 \\
0.0039 \\
0.0065\end{array}$ & $\begin{array}{l}50.4 \\
52.9 \\
51.3\end{array}$ & $\begin{array}{l}4.1 \\
1.7 \\
1.6\end{array}$ & $\begin{array}{l}0.1766 \\
0.1812 \\
0.1787\end{array}$ & $\begin{array}{l}0.0065 \\
0.0039 \\
0.0027\end{array}$ \\
\hline $\begin{array}{l}11953 \\
\text { Front } \\
\text { Center } \\
\text { Back }\end{array}$ & $\begin{array}{l}426 \\
426 \\
421\end{array}$ & $\begin{array}{l}4 \\
5 \\
5\end{array}$ & $\begin{array}{l}3373 \\
3390 \\
3315\end{array}$ & $\begin{array}{l}75 \\
65 \\
58\end{array}$ & $\begin{array}{l}3565 \\
3565 \\
3524\end{array}$ & $\begin{array}{l}31 \\
42 \\
44\end{array}$ & $\begin{array}{l}76.3 \\
81.2 \\
81.1\end{array}$ & $\begin{array}{l}3.6 \\
2.3 \\
2.5\end{array}$ & $\begin{array}{l}0.2174 \\
0.2251 \\
0.2249\end{array}$ & $\begin{array}{l}0.0059 \\
0.0026 \\
0.0028\end{array}$ & $\begin{array}{l}50.6 \\
57.0 \\
51.7\end{array}$ & $\begin{array}{l}2.5 \\
5.1 \\
4.4\end{array}$ & $\begin{array}{l}0.1751 \\
0.1871 \\
0.1778\end{array}$ & $\begin{array}{l}0.0052 \\
0.0077 \\
0.0068\end{array}$ \\
\hline $\begin{array}{l}11954 \\
\text { Front } \\
\text { Center } \\
\text { Back }\end{array}$ & $\begin{array}{l}422 \\
420 \\
418\end{array}$ & $\begin{array}{l}7 \\
7 \\
6\end{array}$ & $\begin{array}{l}3331 \\
3356 \\
3407\end{array}$ & $\begin{array}{l}47 \\
82 \\
57\end{array}$ & $\begin{array}{l}3532 \\
3515 \\
3498\end{array}$ & $\begin{array}{l}55 \\
56 \\
47\end{array}$ & $\begin{array}{l}71.9 \\
73.3 \\
74.4\end{array}$ & $\begin{array}{l}2.7 \\
2.4 \\
3.0\end{array}$ & $\begin{array}{l}0.2119 \\
0.2137 \\
0.2139\end{array}$ & $\begin{array}{l}0.0044 \\
0.0035 \\
0.0046\end{array}$ & $\begin{array}{l}47.5 \\
48.3 \\
46.1\end{array}$ & $\begin{array}{l}2.3 \\
2.3 \\
3.1\end{array}$ & $\begin{array}{l}0.1700 \\
0.1722 \\
0.1696\end{array}$ & $\begin{array}{l}0.0055 \\
0.0040 \\
0.0072\end{array}$ \\
\hline $\begin{array}{l}11955 \\
\text { Front } \\
\text { Center } \\
\text { Back }\end{array}$ & $\begin{array}{l}425 \\
417 \\
422\end{array}$ & $\begin{array}{l}5 \\
8 \\
7\end{array}$ & $\begin{array}{l}3407 \\
3331 \\
3323\end{array}$ & $\begin{array}{l}69 \\
88 \\
85\end{array}$ & $\begin{array}{l}3557 \\
3490 \\
3532\end{array}$ & $\begin{array}{l}42 \\
63 \\
55\end{array}$ & $\begin{array}{l}75.1 \\
74.0 \\
74.6\end{array}$ & $\begin{array}{l}3.3 \\
5.8 \\
6.3\end{array}$ & $\begin{array}{l}0.2152 \\
0.2151 \\
0.2159\end{array}$ & $\begin{array}{l}0.0068 \\
0.0076 \\
0.0080\end{array}$ & $\begin{array}{l}46.4 \\
50.6 \\
48.0\end{array}$ & $\begin{array}{l}3.8 \\
8.0 \\
4.3\end{array}$ & $\begin{array}{l}0.1663 \\
0.1755 \\
0.1709\end{array}$ & $\begin{array}{l}0.0045 \\
0.0111 \\
0.0078\end{array}$ \\
\hline $\begin{array}{l}11956 \\
\text { Front } \\
\text { Center } \\
\text { Back }\end{array}$ & $\begin{array}{l}417 \\
422 \\
422\end{array}$ & $\begin{array}{l}8 \\
6 \\
8\end{array}$ & $\begin{array}{l}3381 \\
3289 \\
3415\end{array}$ & $\begin{array}{l}76 \\
89 \\
47\end{array}$ & $\begin{array}{l}3490 \\
3532 \\
3532\end{array}$ & $\begin{array}{l}63 \\
47 \\
68\end{array}$ & $\begin{array}{l}74.0 \\
77.4 \\
82.7\end{array}$ & $\begin{array}{l}5.5 \\
6.6 \\
9.0\end{array}$ & $\begin{array}{l}0.2152 \\
0.2194 \\
0.2276\end{array}$ & $\begin{array}{l}0.0077 \\
0.0098 \\
0.0100\end{array}$ & $\begin{array}{l}45.7 \\
46.7 \\
45.0\end{array}$ & $\begin{array}{c}10.0 \\
5.1 \\
6.6\end{array}$ & $\begin{array}{l}0.1670 \\
0.1681 \\
0.1648\end{array}$ & $\begin{array}{l}0.0196 \\
0.0101 \\
0.0115\end{array}$ \\
\hline
\end{tabular}


Table E3 cont. Average Physical Property Data by Lane.

\begin{tabular}{|c|c|c|c|c|c|c|c|c|c|c|c|c|c|c|}
\hline Test Methods & & & & & & & IPST & & & & & & & \\
\hline $\begin{array}{c}\text { Sample } \\
\text { ID } \\
(\#) \\
\end{array}$ & $\begin{array}{c}\text { WS } \\
\text { Sheffield } \\
\text { Roughness } \\
\text { (SU) } \\
\end{array}$ & $\begin{array}{l}95 \% \\
\text { C.I. }\end{array}$ & $\begin{array}{c}\text { FS } \\
\text { Sheffield } \\
\text { Roughness } \\
(\mathrm{ml} / \mathrm{min}) \\
\end{array}$ & $\begin{array}{l}95 \% \\
\text { C.I. }\end{array}$ & $\begin{array}{c}\text { WS } \\
\text { Sheffield } \\
\text { Roughness } \\
(\mathrm{ml} / \mathrm{min}) \\
\end{array}$ & $\begin{array}{c}95 \% \\
\text { C.I. }\end{array}$ & $\begin{array}{c}\text { CD } \\
\text { FS Emveco } \\
\text { Micro Dev. }\end{array}$ & $\begin{array}{l}95 \% \\
\text { C.I. }\end{array}$ & $\begin{array}{l}\text { CD } \\
\text { FS Emveco } \\
\text { Micro Ave. }\end{array}$ & $\begin{array}{l}95 \% \\
\text { C.I. }\end{array}$ & $\begin{array}{c}\text { MD } \\
\text { FS Emveco } \\
\text { Micro Dev. }\end{array}$ & $\begin{array}{c}95 \% \\
\text { C.I. }\end{array}$ & $\begin{array}{c}\text { MD } \\
\text { FS Emveco } \\
\text { Micro Ave. }\end{array}$ & $\begin{array}{c}95 \% \\
\text { C.I. }\end{array}$ \\
\hline $\begin{array}{c}\text { Commercial } \\
\text { Medium } \\
107811 \\
\text { Center } \\
\text { Edge } \\
\end{array}$ & & & & & $z$ & & & & & & & & & \\
\hline $\begin{array}{c}\mathbf{1 0 7 8 1 2} \\
\text { Center } \\
\text { Edge }\end{array}$ & & & : & & 3 & & & & & & & & & \\
\hline $\begin{array}{c}107813 \\
\text { Center } \\
\text { Edge } \\
\end{array}$ & & & & & & & & & & & & & & \\
\hline $\begin{array}{l}\text { Commercial } \\
\text { Liner } \\
225111\end{array}$ & & & & & & & & & & & & & & \\
\hline Center & 391 & 4 & 3294 & 65 & 3269 & 37 & 153.5 & 9.5 & 0.3056 & 0.0116 & 125.1 & 10.6 & 0.2733 & 0.0127 \\
\hline Edge & 393 & 6 & 3285 & 44 & 3285 & 53 & 161.3 & 10.8 & 0.3130 & 0.0101 & 118.6 & 8.2 & 0.2650 & 0.0090 \\
\hline $\begin{array}{l}\mathbf{2 2 5 1 2 1} \\
\text { Center } \\
\text { Edoe }\end{array}$ & $\begin{array}{l}391 \\
398\end{array}$ & $\begin{array}{l}7 \\
5\end{array}$ & 3252 & $\begin{array}{l}80 \\
46\end{array}$ & $\begin{array}{l}3269 \\
3329\end{array}$ & $\begin{array}{l}60 \\
40\end{array}$ & $\begin{array}{l}200.6 \\
2136\end{array}$ & $\begin{array}{l}50.6 \\
351\end{array}$ & $\begin{array}{l}0.3276 \\
03377\end{array}$ & $\begin{array}{l}0.0259 \\
0.0184\end{array}$ & $\begin{array}{l}124.0 \\
134.7\end{array}$ & $\begin{array}{l}12.9 \\
147\end{array}$ & $\begin{array}{l}0.2745 \\
0.2817\end{array}$ & $\begin{array}{l}0.0140 \\
0.0120\end{array}$ \\
\hline
\end{tabular}


Table E3 cont. Average Physical Property Data by Lane.

\begin{tabular}{|c|c|c|c|c|c|c|c|c|c|c|}
\hline \multirow[t]{2}{*}{ Test Methods } & \multicolumn{5}{|c|}{ T527 (Diffuse) } & \multicolumn{5}{|c|}{ T527 (Diffuse) } \\
\hline & As Made & & & & & Conditioned & $\mathrm{d}$ Expc & ed to Light & 6 Hours & \\
\hline Sample & Color & $95 \%$ & $\mathrm{~L}^{*}$ & $a^{*}$ & $b^{*}$ & Color & $95 \%$ & $\mathrm{~L}^{*}$ & $a^{*}$ & $b^{*}$ \\
\hline $\begin{array}{l}\text { ID } \\
(\#)\end{array}$ & Brightness & C.I. & Lightness & $\begin{array}{l}\text { +Redness } \\
\text {-Greeness }\end{array}$ & $\begin{array}{c}\text { +Yellowness } \\
\text {-Blueness }\end{array}$ & Brightness & C.I. & Lightness & $\begin{array}{l}\text { +Redness } \\
\text {-Greeness }\end{array}$ & $\begin{array}{l}\text { +Yellowness } \\
\text {-Blueness }\end{array}$ \\
\hline 11926 & & & & & & & & & & \\
\hline Front & 13.96 & 0.06 & 56.95 & 6.42 & 24.07 & 13.06 & 0.13 & 55.09 & 7.36 & 22.83 \\
\hline Center & 14.30 & 0.06 & 57.35 & 6.34 & 23.92 & 13.27 & 0.03 & 55.45 & 7.28 & 22.92 \\
\hline Back & 14.06 & 0.14 & 57.04 & 6.33 & 23.98 & 13.01 & 0.06 & 55.15 & 7.21 & 23.08 \\
\hline 11927 & & & & & & & & & & \\
\hline Front & 13.95 & 0.09 & 56.91 & 6.39 & 24.01 & 13.15 & 0.13 & 55.33 & 7.16 & 23.00 \\
\hline Center & 14.19 & 0.08 & 57.20 & 6.35 & 23.92 & 13.29 & 0.05 & 55.49 & 7.09 & 23.06 \\
\hline Back & 13.95 & 0.12 & 56.90 & 6.38 & 23.97 & 13.00 & 0.13 & 55.14 & 7.15 & 23.13 \\
\hline 11928 & & & & & & & & & & \\
\hline Front & 14.04 & 0.11 & 57.02 & 6.45 & 24.00 & 13.19 & 0.29 & 55.38 & 7.23 & 22.91 \\
\hline Center & 14.20 & 0.05 & 57.28 & 6.29 & 23.94 & 13.30 & 0.05 & 55.54 & 7.13 & 23.02 \\
\hline Back & 14.04 & 0.05 & 56.98 & 6.38 & 23.90 & 13.06 & 0.11 & 55.17 & 7.27 & 22.96 \\
\hline 11930 & & & & & & & & & & \\
\hline Front & 14.09 & 0.13 & 57.05 & 6.41 & 23.84 & 13.21 & 0.21 & 55.23 & 7.17 & 22.74 \\
\hline Center & 14.28 & 0.11 & 57.29 & 6.39 & 23.89 & 13.18 & 0.05 & 55.23 & 7.16 & 22.81 \\
\hline Back & 13.96 & 0.13 & 56.86 & 6.39 & 23.86 & 13.05 & 0.18 & 55.02 & 7.22 & 22.80 \\
\hline 11931 & & & & & & & & & & \\
\hline Front & 14.20 & 0.11 & 57.23 & 6.34 & 23.92 & 13.38 & 0.10 & 55.51 & 7.12 & 22.76 \\
\hline Center & 14.30 & 0.10 & 57.33 & 6.33 & 23.99 & 13.43 & 0.19 & 55.60 & 7.17 & 22.73 \\
\hline Back & 13.98 & 0.08 & 56.92 & 6.34 & 23.97 & 13.27 & 0.08 & 55.29 & 7.30 & 22.63 \\
\hline 11932 & & & & & & & & & & \\
\hline Front & 14.09 & 0.07 & 57.07 & 6.44 & 23.97 & 13.42 & 0.10 & 55.49 & 7.25 & 22.59 \\
\hline Center & 14.15 & 0.04 & 57.16 & 6.33 & 23.95 & 13.38 & 0.05 & 55.47 & 7.10 & 22.68 \\
\hline Back & 13.94 & 0.10 & 56.88 & 6.35 & 23.93 & 13.35 & 0.12 & 55.42 & 7.21 & 22.73 \\
\hline 11933 & & & & & & & & & & \\
\hline Front & 14.13 & 0.16 & 57.07 & 6.31 & 23.81 & 13.45 & 0.11 & 55.44 & 7.13 & 22.52 \\
\hline Center & 14.36 & 0.13 & 57.36 & 6.39 & 23.73 & 13.57 & 0.07 & 55.65 & 7.19 & 22.45 \\
\hline Back & 14.14 & 0.07 & 56.99 & 6.44 & 23.74 & 13.44 & 0.12 & 55.53 & 6.99 & 22.59 \\
\hline 11934 & & & & & & & & & & \\
\hline Front & 14.12 & 0.14 & 57.07 & 6.36 & 23.85 & 13.27 & 0.12 & 55.29 & 7.12 & 22.69 \\
\hline Center & 14.47 & 0.08 & 57.48 & 6.22 & 23.70 & 13.48 & 0.06 & 55.61 & 7.09 & 22.64 \\
\hline Back & 14.17 & 0.05 & 57.05 & 6.30 & 23.69 & 13.21 & 0.07 & 55.28 & 7.16 & 22.78 \\
\hline
\end{tabular}


Table E3 cont. Average Physical Property Data by Lane.

\begin{tabular}{|c|c|c|c|c|c|c|c|c|c|c|}
\hline \multirow[t]{2}{*}{ Test Methods } & \multicolumn{5}{|c|}{ T527 (Diffuse) } & \multicolumn{5}{|c|}{ T527 (Diffuse) } \\
\hline & \multicolumn{5}{|c|}{ As Made } & \multicolumn{5}{|c|}{ Conditioned and Exposed to Light 96 Hours } \\
\hline Sample & Color & $95 \%$ & $\mathrm{~L}^{*}$ & $a^{*}$ & $b^{*}$ & Color & $95 \%$ & $\mathrm{~L}^{*}$ & $a^{*}$ & $\mathrm{~b}^{*}$ \\
\hline $\begin{array}{l}\text { ID } \\
(\#)\end{array}$ & Brightness & C.I. & Lightness & $\begin{array}{l}\text { +Redness } \\
\text {-Greeness }\end{array}$ & $\begin{array}{c}\text { +Yellowness } \\
\text {-Blueness }\end{array}$ & Brightness & C.I. & Lightness & $\begin{array}{l}\text { +Redness } \\
\text {-Greeness }\end{array}$ & $\begin{array}{c}\text { +Yellowness } \\
\text {-Blueness }\end{array}$ \\
\hline 11935 & & & & & & & & & & \\
\hline Front & 14.17 & 0.10 & 57.06 & 6.31 & 23.74 & 13.42 & 0.16 & 55.44 & 7.31 & 22.56 \\
\hline Center & 14.37 & 0.08 & 57.33 & 6.37 & 23.68 & 13.73 & 0.12 & 55.86 & 7.08 & 22.40 \\
\hline Back & 14.21 & 0.02 & 57.06 & 6.47 & 23.62 & 13.58 & 0.18 & 55.72 & 7.07 & 22.49 \\
\hline$\overline{11936}$ & & & & & & & & & & \\
\hline Front & 14.13 & 0.19 & 56.96 & 6.47 & 23.64 & 13.56 & 0.14 & 55.73 & 7.49 & 21.43 \\
\hline Center & 14.32 & 0.09 & 57.24 & 6.31 & 23.63 & 13.79 & 0.08 & 56.03 & 7.43 & 21.41 \\
\hline Back & 14.09 & 0.07 & 56.90 & 6.32 & 23.67 & 13.56 & 0.13 & 55.66 & 7.50 & 21.36 \\
\hline 11937 & & & & & & & & & & \\
\hline Front & & & & & & & & & & \\
\hline Center & & & & & & & & & & \\
\hline Back & & & & & & & & & & \\
\hline 11938 & & & & & & & & & & \\
\hline Front & & & & & - & & & & & \\
\hline Center & & & & & & & & & & \\
\hline Back & & & & & & & & & & \\
\hline 11939 & & & & & & & & & & \\
\hline Front & & & & & & & & & & \\
\hline Center & & & & & & & & & & \\
\hline Back & & & & & & & & & & \\
\hline 11940 & & & & & & & & & & \\
\hline Front & & & & & & & & & & \\
\hline Center & & & & & & & & & & \\
\hline Back & & & & & & & & & & \\
\hline$\overline{11941}$ & & & & & & & & & & \\
\hline Front & & & & & & & & & & \\
\hline Center & & & & & & & & & & \\
\hline Back & & & & & & & & & & \\
\hline 11942 & & & & & & & & & & \\
\hline Front & 15.49 & 0.21 & 59.28 & 5.35 & 24.17 & 14.82 & 0.04 & 57.72 & 7.11 & 21.79 \\
\hline Center & '15.67 & 0.13 & 59.41 & 5.77 & 24.10 & 14.99 & 0.08 & 57.89 & 7.06 & 21.74 \\
\hline Back & 15.61 & 0.10 & 59.36 & 5.76 & 24.15 & 14.94 & 0.17 & 57.88 & 7.03 & 21.81 \\
\hline
\end{tabular}


Table E3 cont. Average Physical Property Data by Lane.

\begin{tabular}{|c|c|c|c|c|c|c|c|c|c|c|}
\hline \multirow[t]{2}{*}{ Test Methods } & \multicolumn{5}{|c|}{ T527 (Diffuse) } & \multicolumn{5}{|c|}{ T527 (Diffuse) } \\
\hline & As Made & & & & & Conditioned & Expo & ed to Light & 6 Hours & \\
\hline Sample & Color & $95 \%$ & $\mathrm{~L}^{*}$ & & $\mathbf{b}^{*}$ & Color & $95 \%$ & $\mathrm{~L}^{*}$ & $a^{*}$ & $b^{*}$ \\
\hline ID & & C.I. & Lightness & +Redness & +Yellowness & & C.I. & Lightness & +Redness & +Yellowness \\
\hline & & & & -Greeness & -Blueness & & & & -Greeness & -Blueness \\
\hline 11943 & & & & & & & & & & \\
\hline Front & 15.67 & 0.07 & 59.36 & 5.86 & 24.20 & 14.94 & 0.10 & 57.84 & 7.10 & 21.77 \\
\hline Center & 15.75 & 0.09 & 59.47 & 5.77 & 24.17 & 14.95 & 0.10 & 57.81 & 7.22 & 21.67 \\
\hline Back & 15.63 & 0.09 & 59.33 & 5.78 & 24.19 & 14.89 & 0.16 & 57.73 & 7.18 & 21.66 \\
\hline 11944 & & & & & & & & & & \\
\hline Front & 15.65 & 0.09 & 59.35 & 5.80 & 24.07 & 14.94 & 0.07 & 57.85 & 7.16 & 21.75 \\
\hline Center & 15.74 & 0.02 & 59.45 & 5.82 & 24.06 & 15.03 & 0.09 & 57.91 & 7.11 & 21.66 \\
\hline Back & 15.72 & 0.14 & 59.43 & 5.80 & 24.04 & 14.92 & 0.15 & 57.76 & 7.19 & 21.58 \\
\hline 11945 & & & & & & & & & & \\
\hline Front & 15.54 & 0.08 & 59.29 & 5.76 & 24.14 & 14.92 & 0.04 & 57.82 & 7.19 & 21.70 \\
\hline Center & 15.20 & 0.07 & 59.45 & 5.85 & 23.91 & 15.04 & 0.04 & 57.93 & 7.11 & 21.67 \\
\hline Back & 15.66 & 0.06 & 59.37 & 5.77 & 24.01 & 14.94 & 0.13 & 57.78 & 7.23 & 21.64 \\
\hline 11946 & & & & & & & & & & \\
\hline Front & 15.49 & 0.24 & 59.00 & 6.17 & 23.80 & 14.93 & 0.25 & 57.79 & 6.87 & 21.74 \\
\hline Center & 15.59 & 0.14 & 59.04 & 6.09 & 23.59 & 15.27 & 0.12 & 58.24 & 6.91 & 21.67 \\
\hline Back & 15.37 & 0.26 & 58.74 & 6.13 & 23.67 & 14.98 & 0.28 & 57.88 & 6.97 & 21.81 \\
\hline 11947 & & & & & & & & & & \\
\hline Front & 15.55 & 0.06 & 59.13 & 5.96 & 23.92 & 14.91 & 0.10 & 57.79 & 7.07 & 21.81 \\
\hline Center & 15.68 & 0.05 & 59.32 & 5.79 & 23.97 & 15.02 & 0.12 & 57.90 & 7.08 & 21.71 \\
\hline Back & 15.64 & 0.18 & 59.23 & 5.80 & 23.84 & 14.95 & 0.25 & 57.78 & 7.06 & 21.71 \\
\hline 11948 & & & & & & & & & & \\
\hline Front & 15.59 & 0.13 & 59.12 & 5.72 & 23.87 & 15.12 & 0.27 & 58.03 & 7.04 & 21.73 \\
\hline Center & 15.69 & 0.06 & 59.25 & 5.74 & 23.79 & 15.47 & 0.12 & 58.42 & 6.83 & 21.58 \\
\hline Back & 15.56 & 0.18 & 59.08 & 5.67 & 23.87 & 15.08 & 0.40 & 57.96 & 6.92 & 21.73 \\
\hline 11949 & & & & & & & & & & \\
\hline Front & 15.52 & 0.27 & 58.95 & 5.65 & 23.83 & 15.12 & 0.30 & 58.02 & 6.93 & 21.93 \\
\hline Center & 15.68 & 0.11 & 59.19 & 5.83 & 23.68 & 15.47 & 0.14 & 58.46 & 6.76 & 21.65 \\
\hline Back & 15.40 & 0.18 & 58.88 & 5.72 & 23.83 & 15.10 & 0.19 & 58.03 & 6.95 & 21.75 \\
\hline 11950 & & & & & & & & & & \\
\hline Front & 13.65 & 0.09 & 56.44 & 6.43 & 24.23 & 13.05 & 0.09 & 55.16 & 7.58 & 21.77 \\
\hline Center & 13.79 & 0.15 & 56.60 & 6.46 & 24.10 & 13.07 & 0.09 & 55.14 & 7.43 & 21.68 \\
\hline Back & 13.59 & 0.21 & 56.30 & 6.60 & 24.06 & 12.99 & 0.14 & 55.00 & 7.59 & 21.67 \\
\hline
\end{tabular}


Table E3 cont. Average Physical Property Data by Lane.

\begin{tabular}{|c|c|c|c|c|c|c|c|c|c|c|}
\hline Test Methods & T527 (Diffus & & & & & T527 (Diffus & & & & \\
\hline $\begin{array}{c}\text { Sample } \\
\text { ID } \\
\text { (\#) }\end{array}$ & $\begin{array}{l}\text { As Made } \\
\text { Color } \\
\text { Brightness }\end{array}$ & $\begin{array}{l}95 \% \\
\text { C.I. }\end{array}$ & $\begin{array}{c}\mathrm{L}^{*} \\
\text { Lightness }\end{array}$ & $\begin{array}{c}a^{*} \\
+ \text { +Redness } \\
\text {-Greeness }\end{array}$ & $\begin{array}{c}\mathrm{b}^{*} \\
+ \text { Yellowness } \\
\text {-Blueness }\end{array}$ & $\begin{array}{c}\text { Conditioned } \\
\text { Color } \\
\text { Brightness }\end{array}$ & $\begin{array}{l}\text { d Expo } \\
95 \% \\
\text { C.I. }\end{array}$ & $\begin{array}{l}\text { ed to Light } \\
L^{*} \\
\text { Lightness }\end{array}$ & $\begin{array}{c}6 \text { Hours } \\
a^{*} \\
+ \text { Redness } \\
\text {-Greeness }\end{array}$ & $\begin{array}{c}b^{*} \\
+ \text { Yellowness } \\
\text {-Blueness } \\
\end{array}$ \\
\hline $\begin{array}{l}11951 \\
\text { Front } \\
\text { Center } \\
\text { Back }\end{array}$ & $\begin{array}{l}13.43 \\
13.71 \\
13.56\end{array}$ & $\begin{array}{l}0.10 \\
0.11 \\
0.15\end{array}$ & $\begin{array}{l}56.09 \\
56.48 \\
56.18\end{array}$ & $\begin{array}{l}6.55 \\
6.44 \\
6.63\end{array}$ & $\begin{array}{l}24.05 \\
23.95 \\
23.94\end{array}$ & $\begin{array}{l}12.99 \\
13.09 \\
12.90\end{array}$ & $\begin{array}{l}0.14 \\
0.06 \\
0.14\end{array}$ & $\begin{array}{l}55.01 \\
55.08 \\
54.83\end{array}$ & $\begin{array}{l}7.64 \\
7.49 \\
7.59\end{array}$ & $\begin{array}{l}21.64 \\
21.57 \\
21.62\end{array}$ \\
\hline $\begin{array}{c}11952 \\
\text { Front } \\
\text { Center } \\
\text { Back } \\
\end{array}$ & $\begin{array}{l}13.56 \\
13.72 \\
13.44\end{array}$ & $\begin{array}{l}0.13 \\
0.08 \\
0.13\end{array}$ & $\begin{array}{l}56.20 \\
56.39 \\
56.00 \\
\end{array}$ & $\begin{array}{l}6.49 \\
6.50 \\
6.65\end{array}$ & $\begin{array}{l}23.98 \\
23.76 \\
23.87 \\
\end{array}$ & $\begin{array}{l}13.04 \\
13.10 \\
12.86\end{array}$ & $\begin{array}{l}0.10 \\
0.07 \\
0.11\end{array}$ & $\begin{array}{l}55.07 \\
55.06 \\
54.79\end{array}$ & $\begin{array}{l}7.51 \\
7.54 \\
7.57\end{array}$ & $\begin{array}{l}21.64 \\
21.51 \\
21.64\end{array}$ \\
\hline $\begin{array}{l}11953 \\
\text { Front } \\
\text { Center } \\
\text { Back }\end{array}$ & $\begin{array}{l}13.46 \\
13.64 \\
13.41\end{array}$ & $\begin{array}{l}0.09 \\
0.06 \\
0.09\end{array}$ & $\begin{array}{l}56.00 \\
56.23 \\
55.83\end{array}$ & $\begin{array}{l}6.57 \\
6.45 \\
6.61\end{array}$ & $\begin{array}{l}23.81 \\
23.73 \\
23.73\end{array}$ & $\begin{array}{l}12.82 \\
13.05 \\
12.83\end{array}$ & $\begin{array}{l}0.12 \\
0.07 \\
0.15\end{array}$ & $\begin{array}{l}54.61 \\
54.88 \\
54.56\end{array}$ & $\begin{array}{l}7.48 \\
7.49 \\
7.56\end{array}$ & $\begin{array}{l}21.35 \\
21.24 \\
21.30\end{array}$ \\
\hline $\begin{array}{l}11954 \\
\text { Front } \\
\text { Center } \\
\text { Back }\end{array}$ & $\begin{array}{l}13.69 \\
14.13 \\
13.62\end{array}$ & $\begin{array}{l}0.09 \\
0.04 \\
0.13\end{array}$ & $\begin{array}{l}56.34 \\
56.83 \\
56.19\end{array}$ & $\begin{array}{l}6.45 \\
6.34 \\
6.27\end{array}$ & $\begin{array}{l}23.83 \\
23.58 \\
23.74\end{array}$ & $\begin{array}{l}13.16 \\
13.47 \\
13.21\end{array}$ & $\begin{array}{l}0.21 \\
0.05 \\
0.19\end{array}$ & $\begin{array}{l}55.04 \\
55.50 \\
55.06\end{array}$ & $\begin{array}{l}7.37 \\
7.16 \\
7.40\end{array}$ & $\begin{array}{l}21.24 \\
21.30 \\
21.19\end{array}$ \\
\hline $\begin{array}{c}11955 \\
\text { Front } \\
\text { Center } \\
\text { Back }\end{array}$ & $\begin{array}{l}13.89 \\
14.25 \\
13.84\end{array}$ & $\begin{array}{l}0.15 \\
0.06 \\
0.08\end{array}$ & $\begin{array}{l}56.54 \\
57.06 \\
56.47\end{array}$ & $\begin{array}{l}6.32 \\
6.13 \\
6.23\end{array}$ & $\begin{array}{l}23.73 \\
23.64 \\
23.68\end{array}$ & $\begin{array}{l}13.32 \\
13.50 \\
13.36\end{array}$ & $\begin{array}{l}0.19 \\
0.12 \\
0.14\end{array}$ & $\begin{array}{l}55.28 \\
55.50 \\
55.26\end{array}$ & $\begin{array}{l}7.24 \\
7.23 \\
7.30\end{array}$ & $\begin{array}{l}21.27 \\
21.27 \\
21.19\end{array}$ \\
\hline $\begin{array}{l}11956 \\
\text { Front } \\
\text { Center } \\
\text { Back }\end{array}$ & $\begin{array}{l}13.98 \\
14.26 \\
14.22\end{array}$ & $\begin{array}{l}0.12 \\
0.12 \\
0.07\end{array}$ & $\begin{array}{l}56.64 \\
56.99 \\
56.85\end{array}$ & $\begin{array}{l}6.09 \\
6.11 \\
6.07\end{array}$ & $\begin{array}{l}23.58 \\
23.55 \\
23.50\end{array}$ & $\begin{array}{l}13.36 \\
13.48 \\
13.47\end{array}$ & $\begin{array}{l}0.19 \\
0.06 \\
0.12\end{array}$ & $\begin{array}{l}55.27 \\
55.43 \\
55.35\end{array}$ & $\begin{array}{l}7.26 \\
7.16 \\
7.18\end{array}$ & $\begin{array}{l}21.14 \\
21.18 \\
21.03\end{array}$ \\
\hline
\end{tabular}


Table E3 cont. Average Physical Property Data by Lane.

\begin{tabular}{|c|c|c|c|c|c|c|c|c|c|c|}
\hline \multirow[t]{2}{*}{ Test Methods } & \multicolumn{5}{|c|}{ T527 (Diffuse) } & \multicolumn{5}{|c|}{ T527 (Diffuse) } \\
\hline & \multicolumn{5}{|c|}{ As Made } & \multicolumn{5}{|c|}{ Conditioned and Exposed to Light 96 Hours } \\
\hline Sample & Color & $95 \%$ & $L^{*}$ & $a^{*}$ & $b^{*}$ & Color & $95 \%$ & $\mathrm{~L}^{*}$ & $a^{*}$ & $b^{*}$ \\
\hline $\begin{array}{l}\text { ID } \\
(\#)\end{array}$ & Brightness & C.I. & Lightness & $\begin{array}{l}\text { +Redness } \\
\text {-Greeness }\end{array}$ & $\begin{array}{l}\text { +Yellowness } \\
\text {-Blueness }\end{array}$ & Brightness & C.I. & Lightness & $\begin{array}{l}\text { +Redness } \\
\text {-Greeness }\end{array}$ & $\begin{array}{c}\text { +Yellowness } \\
\text {-Blueness }\end{array}$ \\
\hline \\
\hline \multicolumn{11}{|l|}{ Medium } \\
\hline \multicolumn{11}{|l|}{107811} \\
\hline \multicolumn{11}{|l|}{ Center } \\
\hline \multicolumn{11}{|l|}{ Edge } \\
\hline \multicolumn{11}{|l|}{107812} \\
\hline \multicolumn{11}{|l|}{ Center } \\
\hline \multicolumn{11}{|l|}{ Edge } \\
\hline \multicolumn{11}{|l|}{107813} \\
\hline \multicolumn{11}{|l|}{ Center } \\
\hline \multicolumn{11}{|l|}{ Edge } \\
\hline \multicolumn{11}{|l|}{107814} \\
\hline \multicolumn{11}{|l|}{ Center } \\
\hline \multicolumn{11}{|l|}{ Edge } \\
\hline \multicolumn{11}{|l|}{107816} \\
\hline \multicolumn{11}{|l|}{ Center } \\
\hline \multicolumn{11}{|l|}{ Edge } \\
\hline \multirow{2}{*}{\multicolumn{11}{|c|}{$\begin{array}{l}\text { Commercial } \\
\text { Liner }\end{array}$}} \\
\hline & & & & & & & & & & \\
\hline \multicolumn{11}{|l|}{225111} \\
\hline Center & 16.03 & 0.07 & 58.63 & 6.47 & 21.96 & 15.97 & 0.05 & 58.83 & 6.56 & 21.14 \\
\hline Edge & 16.01 & 0.06 & 58.62 & 6.49 & 21.97 & 15.91 & 0.04 & 58.75 & 6.51 & 21.13 \\
\hline 225121 & & & & & & & & & & \\
\hline Center & 16.16 & 0.09 & 58.86 & 6.31 & 22.11 & 15.92 & 0.06 & 58.71 & 6.54 & 20.95 \\
\hline Edge & 16.12 & 0.07 & 58.80 & 6.41 & 22.08 & 15.88 & 0.07 & 58.72 & 6.53 & 21.04 \\
\hline
\end{tabular}


Table E3 cont. Average Physical Property Data by Lane.

\begin{tabular}{|c|c|c|c|c|c|c|c|c|c|c|c|c|c|c|c|}
\hline Test Methods & T524 (Direct & onal) & & & & T480 & & $\mathrm{T} 441$ & & & & T403 & & & \\
\hline $\begin{array}{l}\text { Sample } \\
\text { ID } \\
\text { (\#) }\end{array}$ & $\begin{array}{l}\text { Conditioned } \\
\text { Color } \\
\text { Brightness }\end{array}$ & $\begin{array}{l}\text { and Ex } \\
95 \% \\
\text { C.I. }\end{array}$ & $\begin{array}{l}\text { osed to Lig } \\
\qquad L^{*} \\
\text { Lightness }\end{array}$ & $\begin{array}{l}\text { 96 Hours } \\
a^{*} \\
+ \text { Redness } \\
\text {-Greeness }\end{array}$ & $\begin{array}{c}\text { b* } \\
+ \text { Yellowness } \\
\text {-Blueness }\end{array}$ & Gloss & $\begin{array}{l}95 \% \\
\text { C.I. }\end{array}$ & $\begin{array}{c}\text { FS Cobb } \\
\text { Sizing } \\
\left(\mathrm{g} / \mathrm{m}^{\wedge} 2\right)\end{array}$ & $\begin{array}{l}95 \% \\
\text { C.I. }\end{array}$ & $\begin{array}{l}\text { WS Cobb } \\
\text { Sizing } \\
\left(\mathrm{g} / \mathrm{m}^{\wedge} 2\right)\end{array}$ & $\begin{array}{l}95 \% \\
\text { C.I. }\end{array}$ & $\begin{array}{l}\text { Burst } \\
\text { (psi) }\end{array}$ & $\begin{array}{c}95 \% \\
\text { C.I. }\end{array}$ & $\begin{array}{l}\text { Burst } \\
(\mathrm{kPa})\end{array}$ & $\begin{array}{c}95 \% \\
\text { C.I. }\end{array}$ \\
\hline $\begin{array}{l}11926 \\
\text { Front } \\
\text { Center } \\
\text { Back }\end{array}$ & $\begin{array}{l}13.32 \\
13.59 \\
13.51\end{array}$ & $\begin{array}{l}0.22 \\
0.26 \\
0.30\end{array}$ & $\begin{array}{l}55.74 \\
56.17 \\
55.99 \\
\end{array}$ & $\begin{array}{l}7.46 \\
7.32 \\
7.43 \\
\end{array}$ & $\begin{array}{l}22.15 \\
22.14 \\
22.14\end{array}$ & $\begin{array}{l}13.00 \\
12.52 \\
12.61\end{array}$ & $\begin{array}{l}0.21 \\
0.35 \\
0.30\end{array}$ & $\begin{array}{l}294.42 \\
304.54 \\
315.11\end{array}$ & $\begin{array}{c}29.64 \\
5.67 \\
15.53 \\
\end{array}$ & $\begin{array}{l}289.50 \\
293.92 \\
308.16\end{array}$ & $\begin{array}{c}6.10 \\
12.36 \\
19.96\end{array}$ & $\begin{array}{l}78.04 \\
76.84 \\
80.92 \\
\end{array}$ & $\begin{array}{l}5.06 \\
3.34 \\
4.93\end{array}$ & $\begin{array}{l}538.1 \\
529.8 \\
557.9\end{array}$ & $\begin{array}{l}34.9 \\
23.0 \\
34.0\end{array}$ \\
\hline $\begin{array}{l}11927 \\
\text { Front } \\
\text { Center } \\
\text { Back }\end{array}$ & $\begin{array}{l}13.48 \\
13.73 \\
13.44 \\
\end{array}$ & $\begin{array}{l}0.34 \\
0.32 \\
0.42 \\
\end{array}$ & $\begin{array}{l}56.00 \\
56.23 \\
55.92 \\
\end{array}$ & $\begin{array}{l}7.42 \\
7.30 \\
7.37 \\
\end{array}$ & $\begin{array}{l}22.22 \\
22.07 \\
22.21 \\
\end{array}$ & $\begin{array}{l}13.09 \\
13.09 \\
13.26 \\
\end{array}$ & $\begin{array}{l}0.40 \\
0.63 \\
0.57 \\
\end{array}$ & $\begin{array}{l}293.53 \\
295.69 \\
292.20 \\
\end{array}$ & $\begin{array}{l}5.26 \\
6.96 \\
5.98 \\
\end{array}$ & $\begin{array}{l}292.28 \\
297.37 \\
295.33 \\
\end{array}$ & $\begin{array}{c}10.70 \\
15.80 \\
4.78 \\
\end{array}$ & $\begin{array}{l}79.00 \\
75.24 \\
75.92 \\
\end{array}$ & $\begin{array}{l}5.46 \\
5.56 \\
5.19 \\
\end{array}$ & $\begin{array}{l}544.7 \\
518.8 \\
523.4 \\
\end{array}$ & $\begin{array}{l}37.6 \\
38.3 \\
35.8 \\
\end{array}$ \\
\hline $\begin{array}{c}11928 \\
\text { Front } \\
\text { Center } \\
\text { Back }\end{array}$ & $\begin{array}{l}13.28 \\
13.60 \\
13.36 \\
\end{array}$ & $\begin{array}{l}0.32 \\
0.21 \\
0.31 \\
\end{array}$ & $\begin{array}{l}55.72 \\
56.18 \\
55.78 \\
\end{array}$ & $\begin{array}{l}7.38 \\
7.28 \\
7.38 \\
\end{array}$ & $\begin{array}{l}22.22 \\
22.26 \\
22.16\end{array}$ & $\begin{array}{l}12.75 \\
12.96 \\
13.09\end{array}$ & $\begin{array}{l}0.30 \\
0.38 \\
0.33\end{array}$ & $\begin{array}{l}290.95 \\
295.82 \\
288.47\end{array}$ & $\begin{array}{l}17.06 \\
12.28 \\
11.74 \\
\end{array}$ & $\begin{array}{l}283.72 \\
300.51 \\
297.59\end{array}$ & $\begin{array}{c}16.99 \\
5.93 \\
13.22\end{array}$ & $\begin{array}{l}77.63 \\
76.14 \\
80.02 \\
\end{array}$ & $\begin{array}{l}3.51 \\
7.17 \\
4.18\end{array}$ & $\begin{array}{l}535.3 \\
525.0 \\
551.7\end{array}$ & $\begin{array}{l}24.2 \\
49.4 \\
28.8\end{array}$ \\
\hline $\begin{array}{c}11930 \\
\text { Front } \\
\text { Center } \\
\text { Back }\end{array}$ & $\begin{array}{l}13.40 \\
13.55 \\
13.46 \\
\end{array}$ & $\begin{array}{l}0.26 \\
0.37 \\
0.33\end{array}$ & $\begin{array}{l}55.75 \\
55.94 \\
55.77 \\
\end{array}$ & $\begin{array}{l}7.37 \\
7.39 \\
7.23 \\
\end{array}$ & $\begin{array}{l}22.00 \\
21.91 \\
21.91 \\
\end{array}$ & $\begin{array}{l}12.04 \\
12.04 \\
11.98 \\
\end{array}$ & $\begin{array}{l}0.32 \\
0.32 \\
0.35\end{array}$ & $\begin{array}{l}296.85 \\
313.61 \\
294.27\end{array}$ & $\begin{array}{c}11.34 \\
4.92 \\
9.79 \\
\end{array}$ & $\begin{array}{l}297.17 \\
306.98 \\
301.32 \\
\end{array}$ & $\begin{array}{c}9.71 \\
11.91 \\
14.32\end{array}$ & $\begin{array}{l}78.19 \\
75.78 \\
79.70 \\
\end{array}$ & $\begin{array}{l}3.65 \\
4.98 \\
3.21 \\
\end{array}$ & $\begin{array}{l}539.1 \\
522.5 \\
549.6 \\
\end{array}$ & $\begin{array}{l}25.2 \\
34.3 \\
22.1 \\
\end{array}$ \\
\hline $\begin{array}{l}11931 \\
\text { Front } \\
\text { Center } \\
\text { Back }\end{array}$ & $\begin{array}{l}13.28 \\
13.46 \\
13.33 \\
\end{array}$ & $\begin{array}{l}0.23 \\
0.39 \\
0.27\end{array}$ & $\begin{array}{l}55.60 \\
55.91 \\
55.67 \\
\end{array}$ & $\begin{array}{l}7.33 \\
7.37 \\
7.41 \\
\end{array}$ & $\begin{array}{l}22.02 \\
22.08 \\
22.05 \\
\end{array}$ & $\begin{array}{l}11.85 \\
12.16 \\
12.08 \\
\end{array}$ & $\begin{array}{l}0.32 \\
0.28 \\
0.34\end{array}$ & $\begin{array}{l}277.85 \\
291.66 \\
286.82 \\
\end{array}$ & $\begin{array}{l}10.60 \\
25.32 \\
11.36 \\
\end{array}$ & $\begin{array}{l}295.57 \\
299.68 \\
275.96 \\
\end{array}$ & $\begin{array}{c}12.56 \\
19.98 \\
8.62 \\
\end{array}$ & $\begin{array}{l}80.29 \\
76.04 \\
79.48 \\
\end{array}$ & $\begin{array}{l}3.38 \\
5.52 \\
5.56 \\
\end{array}$ & $\begin{array}{l}553.6 \\
524.3 \\
548.0 \\
\end{array}$ & $\begin{array}{l}23.3 \\
38.1 \\
38.3 \\
\end{array}$ \\
\hline $\begin{array}{c}11932 \\
\text { Front } \\
\text { Center } \\
\text { Back }\end{array}$ & $\begin{array}{l}13.47 \\
13.76 \\
13.61 \\
\end{array}$ & $\begin{array}{l}0.29 \\
0.19 \\
0.32\end{array}$ & $\begin{array}{l}55.84 \\
56.20 \\
56.03 \\
\end{array}$ & $\begin{array}{l}7.26 \\
7.31 \\
7.22 \\
\end{array}$ & $\begin{array}{l}21.94 \\
21.86 \\
21.93\end{array}$ & $\begin{array}{l}12.20 \\
12.13 \\
12.07 \\
\end{array}$ & $\begin{array}{l}0.33 \\
0.36 \\
0.23\end{array}$ & $\begin{array}{l}277.48 \\
286.67 \\
300.98 \\
\end{array}$ & $\begin{array}{c}17.98 \\
24.45 \\
7.15 \\
\end{array}$ & $\begin{array}{l}271.92 \\
311.50 \\
313.66 \\
\end{array}$ & $\begin{array}{c}11.88 \\
8.35 \\
11.10 \\
\end{array}$ & $\begin{array}{l}78.64 \\
74.72 \\
79.05 \\
\end{array}$ & $\begin{array}{l}4.17 \\
2.49 \\
4.00 \\
\end{array}$ & $\begin{array}{l}542.2 \\
515.2 \\
545.0 \\
\end{array}$ & $\begin{array}{l}28.7 \\
17.2 \\
27.6 \\
\end{array}$ \\
\hline $\begin{array}{c}11933 \\
\text { Front } \\
\text { Center } \\
\text { Back }\end{array}$ & $\begin{array}{l}13.71 \\
13.75 \\
13.59 \\
\end{array}$ & $\begin{array}{l}0.48 \\
0.18 \\
0.33\end{array}$ & $\begin{array}{l}56.06 \\
56.18 \\
55.91 \\
\end{array}$ & $\begin{array}{l}7.25 \\
7.15 \\
7.28\end{array}$ & $\begin{array}{l}21.73 \\
21.85 \\
21.82\end{array}$ & $\begin{array}{l}13.83 \\
13.67 \\
13.40\end{array}$ & $\begin{array}{l}0.39 \\
0.35 \\
0.51\end{array}$ & $\begin{array}{l}301.67 \\
300.93 \\
298.23\end{array}$ & $\begin{array}{c}13.82 \\
6.70 \\
12.53\end{array}$ & $\begin{array}{l}294.59 \\
296.33 \\
293.33\end{array}$ & $\begin{array}{c}22.14 \\
5.91 \\
10.40\end{array}$ & $\begin{array}{l}70.98 \\
74.72 \\
75.02 \\
\end{array}$ & $\begin{array}{l}4.44 \\
5.95 \\
3.44 \\
\end{array}$ & $\begin{array}{l}489.4 \\
515.2 \\
517.2 \\
\end{array}$ & $\begin{array}{l}30.6 \\
41.0 \\
23.7\end{array}$ \\
\hline $\begin{array}{l}11934 \\
\text { Front } \\
\text { Center } \\
\text { Back }\end{array}$ & $\begin{array}{l}13.54 \\
13.90 \\
13.67\end{array}$ & $\begin{array}{l}0.37 \\
0.16 \\
0.46\end{array}$ & $\begin{array}{l}55.95 \\
56.33 \\
56.09\end{array}$ & $\begin{array}{l}7.27 \\
7.21 \\
7.25\end{array}$ & $\begin{array}{l}21.88 \\
21.75 \\
21.90\end{array}$ & $\begin{array}{l}14.13 \\
12.77 \\
13.25\end{array}$ & $\begin{array}{l}0.30 \\
0.92 \\
0.41\end{array}$ & $\begin{array}{l}308.40 \\
311.40 \\
304.79\end{array}$ & $\begin{array}{l}5.81 \\
11.46 \\
19.43\end{array}$ & $\begin{array}{l}294.39 \\
316.86 \\
317.01\end{array}$ & $\begin{array}{l}21.11 \\
10.91 \\
12.64\end{array}$ & $\begin{array}{l}74.90 \\
75.22 \\
75.58 \\
\end{array}$ & $\begin{array}{l}5.10 \\
4.37 \\
5.04\end{array}$ & $\begin{array}{l}516.4 \\
518.6 \\
521.1 \\
\end{array}$ & $\begin{array}{l}35.1 \\
30.1 \\
34.8\end{array}$ \\
\hline
\end{tabular}


Table E3 cont. Average Physical Property Data by Lane.

\begin{tabular}{|c|c|c|c|c|c|c|c|c|c|c|c|c|c|c|c|}
\hline Test Methods & T524 (Direct & onal) & & & & $\mathrm{T} 480$ & & $\mathrm{~T} 441$ & & & & $\mathrm{~T} 403$ & & & \\
\hline $\begin{array}{c}\text { Sample } \\
\text { ID } \\
\text { (\#) }\end{array}$ & $\begin{array}{c}\text { Conditioned } \\
\text { Color } \\
\text { Brightness }\end{array}$ & $\begin{array}{c}\text { nd Ex } \\
95 \% \\
\text { C.I. }\end{array}$ & $\begin{array}{l}\text { osed to Ligl } \\
\text { L* } \\
\text { Lightness }\end{array}$ & $\begin{array}{l}96 \text { Hours } \\
a^{*} \\
+ \text { Redness } \\
\text {-Greeness }\end{array}$ & $\begin{array}{c}b^{*} \\
+ \text { Yellowness } \\
\text {-Blueness }\end{array}$ & Gloss & $\begin{array}{l}95 \% \\
\text { C.I. }\end{array}$ & $\begin{array}{c}\text { FS Cobb } \\
\text { Sizing } \\
\left(\mathrm{g} / \mathrm{m}^{\wedge} 2\right)\end{array}$ & $\begin{array}{c}95 \% \\
\text { C.I. }\end{array}$ & $\begin{array}{c}\text { WS Cobb } \\
\text { Sizing } \\
\left(\mathrm{g} / \mathrm{m}^{\wedge} 2\right)\end{array}$ & $\begin{array}{l}95 \% \\
\text { C.I. }\end{array}$ & $\begin{array}{l}\text { Burst } \\
\text { (psi) }\end{array}$ & $\begin{array}{c}95 \% \\
\text { C.I. }\end{array}$ & $\begin{array}{l}\text { Burst } \\
(\mathrm{kPa})\end{array}$ & $\begin{array}{c}95 \% \\
\text { C.I. }\end{array}$ \\
\hline $\begin{array}{l}11935 \\
\text { Front } \\
\text { Center } \\
\text { Back }\end{array}$ & $\begin{array}{l}13.75 \\
13.95 \\
13.57\end{array}$ & $\begin{array}{l}0.46 \\
0.29 \\
0.32\end{array}$ & $\begin{array}{l}56.18 \\
56.39 \\
55.96\end{array}$ & $\begin{array}{l}7.16 \\
7.14 \\
7.24\end{array}$ & $\begin{array}{l}21.85 \\
21.75 \\
21.90\end{array}$ & $\begin{array}{l}13.60 \\
13.20 \\
12.93\end{array}$ & $\begin{array}{l}0.55 \\
0.25 \\
0.35\end{array}$ & $\begin{array}{l}292.84 \\
312.21 \\
297.83\end{array}$ & $\begin{array}{l}11.55 \\
9.61 \\
9.03\end{array}$ & $\begin{array}{l}287.24 \\
297.81 \\
301.15\end{array}$ & $\begin{array}{l}13.19 \\
8.31 \\
9.47\end{array}$ & $\begin{array}{l}79.68 \\
77.37 \\
76.98\end{array}$ & $\begin{array}{l}5.03 \\
5.78 \\
4.64 \\
\end{array}$ & $\begin{array}{l}549.4 \\
533.4 \\
530.7\end{array}$ & $\begin{array}{l}34.7 \\
39.8 \\
32.0\end{array}$ \\
\hline $\begin{array}{c}11936 \\
\text { Front } \\
\text { Center } \\
\text { Back }\end{array}$ & $\begin{array}{l}13.66 \\
14.08 \\
13.59\end{array}$ & $\begin{array}{l}0.13 \\
0.31 \\
0.39\end{array}$ & $\begin{array}{l}56.25 \\
56.75 \\
56.03 \\
\end{array}$ & $\begin{array}{l}7.11 \\
7.01 \\
7.17\end{array}$ & $\begin{array}{l}22.07 \\
22.02 \\
22.01\end{array}$ & $\begin{array}{l}13.71 \\
13.33 \\
13.41\end{array}$ & $\begin{array}{l}0.33 \\
0.32 \\
0.25\end{array}$ & $\begin{array}{l}299.41 \\
290.07 \\
277.63\end{array}$ & $\begin{array}{l}15.43 \\
21.57 \\
12.93\end{array}$ & $\begin{array}{l}264.30 \\
280.70 \\
267.11\end{array}$ & $\begin{array}{c}12.81 \\
12.67 \\
9.48\end{array}$ & $\begin{array}{l}75.51 \\
74.77 \\
75.67\end{array}$ & $\begin{array}{l}5.81 \\
5.13 \\
5.38\end{array}$ & $\begin{array}{l}520.7 \\
515.5 \\
521.7\end{array}$ & $\begin{array}{l}40.1 \\
35.3 \\
37.1\end{array}$ \\
\hline $\begin{array}{l}11937 \\
\text { Front } \\
\text { Center } \\
\text { Back }\end{array}$ & & & & & & & & & & & & & & & \\
\hline $\begin{array}{l}11938 \\
\text { Front } \\
\text { Center } \\
\text { Back }\end{array}$ & : & & & & & & & & & & & & & & \\
\hline $\begin{array}{l}11939 \\
\text { Front } \\
\text { Center } \\
\text { Back }\end{array}$ & & & & & & & & & & & & & & & \\
\hline $\begin{array}{c}11940 \\
\text { Front } \\
\text { Center } \\
\text { Back }\end{array}$ & & & & & & & & & & & & & & & \\
\hline $\begin{array}{c}11941 \\
\text { Front } \\
\text { Center } \\
\text { Back }\end{array}$ & & & & & & & & & & & & & & & \\
\hline $\begin{array}{l}11942 \\
\text { Front }\end{array}$ & 14.94 & 0.42 & 58.16 & 6.81 & 22.40 & 7.07 & 0.12 & 372.22 & 15.77 & 361.84 & 15.00 & 70.20 & 3.82 & 484.0 & 26.4 \\
\hline Center & 15.40 & 0.38 & 58.69 & 6.56 & 22.25 & 7.14 & 0.35 & 374.45 & 6.88 & 382.12 & 15.01 & 65.26 & 6.20 & 449.9 & 42.8 \\
\hline Back & 15.26 & 0.20 & 58.56 & 6.61 & 22.35 & 8.10 & 0.44 & 372.02 & 3.89 & 355.06 & 8.69 & 66.16 & 4.69 & 456.2 & 32.4 \\
\hline
\end{tabular}


Table E3 cont. Average Physical Property Data by Lane.

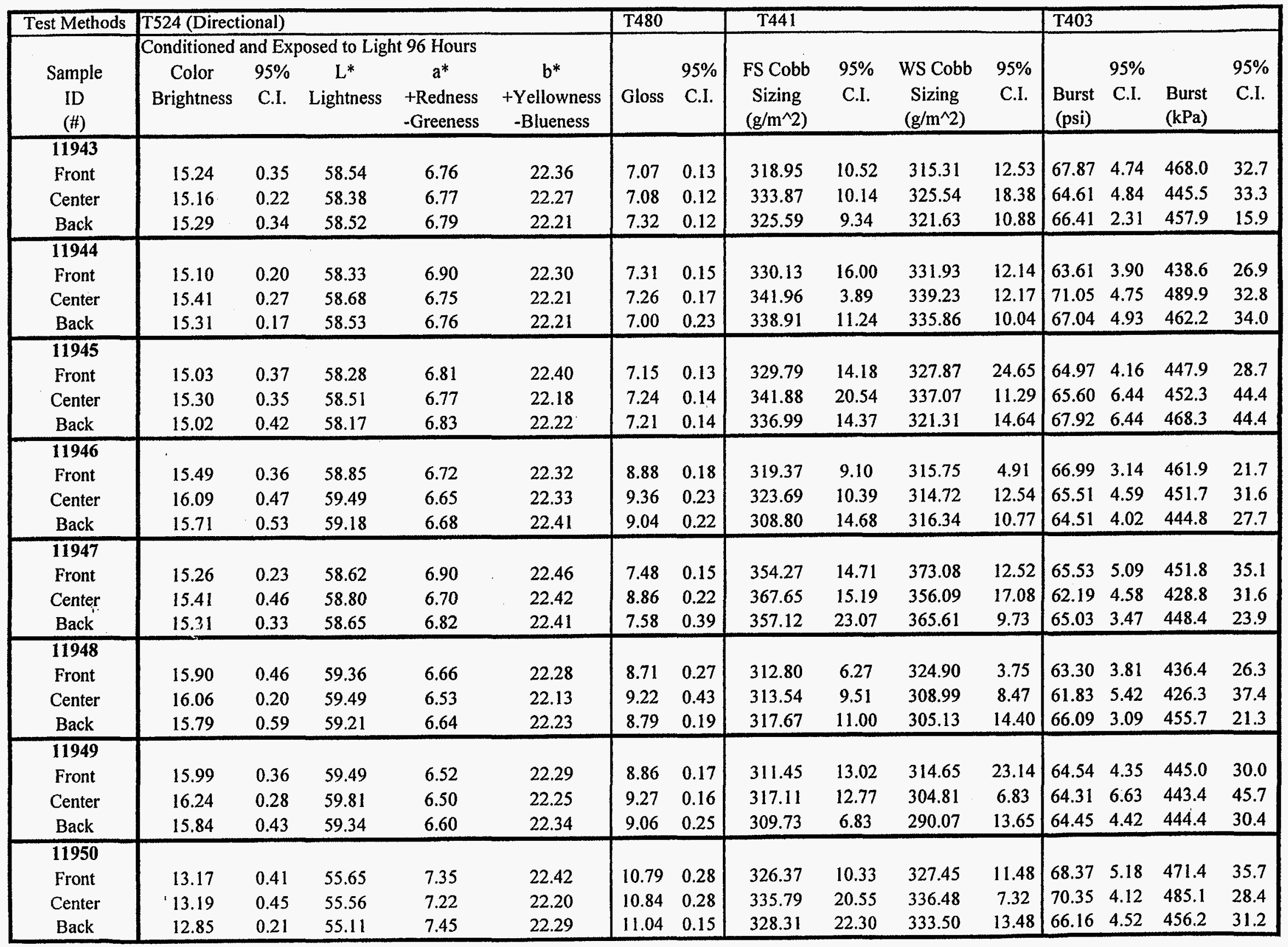


Table E3 cont. Average Physical Property Data by Lane.

\begin{tabular}{|c|c|c|c|c|c|c|c|c|c|c|c|c|c|c|c|}
\hline Test Methods & T524 (Direct & onal) & & & & $\mathrm{T} 480$ & & T441 & & & & $\mathrm{T} 403$ & & & \\
\hline $\begin{array}{l}\text { Sample } \\
\text { ID } \\
(\#)\end{array}$ & $\begin{array}{l}\text { Conditioned } \\
\text { Color } \\
\text { Brightness }\end{array}$ & $\begin{array}{l}\text { nd Exp } \\
95 \% \\
\text { C.I. }\end{array}$ & $\begin{array}{l}\text { osed to Lig } \\
L^{*} \\
\text { Lightness }\end{array}$ & $\begin{array}{c}\text { t96 Hours } \\
a^{*} \\
+ \text { Redness } \\
\text {-Greeness }\end{array}$ & $\begin{array}{c}b^{*} \\
+ \text { Yellowness } \\
\text {-Blueness } \\
\end{array}$ & Gloss & $\begin{array}{c}95 \% \\
\text { C.I. }\end{array}$ & $\begin{array}{l}\text { FS Cobb } \\
\text { Sizing } \\
\left(g / m^{\wedge} 2\right)\end{array}$ & $\begin{array}{c}95 \% \\
\text { C.I. }\end{array}$ & $\begin{array}{l}\text { WS Cobb } \\
\text { Sizing } \\
\left(g / m^{\wedge} 2\right)\end{array}$ & $\begin{array}{c}95 \% \\
\text { C.I. }\end{array}$ & $\begin{array}{l}\text { Burst } \\
\text { (psi) }\end{array}$ & $\begin{array}{l}95 \% \\
\text { C.I. }\end{array}$ & $\begin{array}{l}\text { Burst } \\
(\mathrm{kPa})\end{array}$ & $\begin{array}{c}95 \% \\
\text { C.I. }\end{array}$ \\
\hline $\begin{array}{l}11951 \\
\text { Front } \\
\text { Center } \\
\text { Back }\end{array}$ & $\begin{array}{l}13.22 \\
13.27 \\
13.03\end{array}$ & $\begin{array}{l}0.30 \\
0.21 \\
0.19\end{array}$ & $\begin{array}{l}55.62 \\
55.61 \\
55.28\end{array}$ & $\begin{array}{l}7.30 \\
7.17 \\
7.38 \\
\end{array}$ & $\begin{array}{l}22.24 \\
22.09 \\
22.11\end{array}$ & $\begin{array}{l}11.43 \\
11.68 \\
11.95 \\
\end{array}$ & $\begin{array}{l}0.52 \\
0.24 \\
0.19 \\
\end{array}$ & $\begin{array}{l}313.22 \\
326.91 \\
311.35 \\
\end{array}$ & $\begin{array}{c}4.40 \\
7.64 \\
14.36 \\
\end{array}$ & $\begin{array}{l}332.44 \\
313.25 \\
312.48 \\
\end{array}$ & $\begin{array}{l}15.96 \\
19.55 \\
12.65 \\
\end{array}$ & $\begin{array}{l}70.85 \\
68.12 \\
68.66 \\
\end{array}$ & $\begin{array}{l}4.51 \\
4.36 \\
4.30 \\
\end{array}$ & $\begin{array}{l}488.5 \\
469.7 \\
473.4 \\
\end{array}$ & $\begin{array}{l}31.1 \\
30.1 \\
29.6 \\
\end{array}$ \\
\hline $\begin{array}{l}11952 \\
\text { Front } \\
\text { Center } \\
\text { Back }\end{array}$ & $\begin{array}{l}12.99 \\
13.19 \\
12.87\end{array}$ & $\begin{array}{l}0.17 \\
0.31 \\
0.35\end{array}$ & $\begin{array}{l}55.24 \\
55.47 \\
55.10\end{array}$ & $\begin{array}{l}7.39 \\
7.25 \\
7.44 \\
\end{array}$ & $\begin{array}{l}22.14 \\
22.04 \\
22.22\end{array}$ & $\begin{array}{l}12.47 \\
12.30 \\
12.66\end{array}$ & $\begin{array}{l}0.44 \\
0.19 \\
0.33\end{array}$ & $\begin{array}{l}297.98 \\
315.46 \\
306.51 \\
\end{array}$ & $\begin{array}{c}16.32 \\
8.22 \\
12.78\end{array}$ & $\begin{array}{l}302.09 \\
314.99 \\
312.02 \\
\end{array}$ & $\begin{array}{c}9.28 \\
23.56 \\
9.82\end{array}$ & $\begin{array}{l}68.25 \\
63.92 \\
71.16\end{array}$ & $\begin{array}{l}3.13 \\
2.04 \\
6.69\end{array}$ & $\begin{array}{l}470.6 \\
440.7 \\
490.7\end{array}$ & $\begin{array}{l}21.6 \\
14.1 \\
46.1\end{array}$ \\
\hline $\begin{array}{l}11953 \\
\text { Front } \\
\text { Center } \\
\text { Back }\end{array}$ & $\begin{array}{l}12.98 \\
13.20 \\
13.06\end{array}$ & $\begin{array}{l}0.21 \\
0.41 \\
0.43\end{array}$ & $\begin{array}{l}55.11 \\
55.43 \\
55.20\end{array}$ & $\begin{array}{l}7.29 \\
7.18 \\
7.30 \\
\end{array}$ & $\begin{array}{l}21.95 \\
21.97 \\
21.92 \\
\end{array}$ & $\begin{array}{l}13.90 \\
13.19 \\
13.50 \\
\end{array}$ & $\begin{array}{l}0.35 \\
0.29 \\
0.38\end{array}$ & $\begin{array}{l}300.98 \\
322.78 \\
310.32 \\
\end{array}$ & $\begin{array}{c}9.37 \\
19.62 \\
6.25\end{array}$ & $\begin{array}{l}292.65 \\
314.82 \\
308.13 \\
\end{array}$ & $\begin{array}{c}13.26 \\
9.37 \\
22.96\end{array}$ & $\begin{array}{l}71.27 \\
69.86 \\
68.21 \\
\end{array}$ & $\begin{array}{l}5.61 \\
5.75 \\
4.52 \\
\end{array}$ & $\begin{array}{l}491.4 \\
481.7 \\
470.3 \\
\end{array}$ & $\begin{array}{l}38.7 \\
39.7 \\
31.2 \\
\end{array}$ \\
\hline $\begin{array}{c}11954 \\
\text { Front } \\
\text { Center } \\
\text { Back }\end{array}$ & $\begin{array}{l}13.11 \\
13.57 \\
13.20\end{array}$ & $\begin{array}{l}0.51 \\
0.26 \\
0.25\end{array}$ & $\begin{array}{l}55.34 \\
56.01 \\
55.43\end{array}$ & $\begin{array}{l}7.11 \\
6.98 \\
7.19\end{array}$ & $\begin{array}{l}21.99 \\
22.02 \\
21.93\end{array}$ & $\begin{array}{l}15.53 \\
14.39 \\
14.98 \\
\end{array}$ & $\begin{array}{l}0.29 \\
0.24 \\
0.35\end{array}$ & $\begin{array}{l}302.06 \\
308.53 \\
305.92\end{array}$ & $\begin{array}{l}20.66 \\
12.70 \\
13.21\end{array}$ & $\begin{array}{l}298.32 \\
315.88 \\
321.14\end{array}$ & $\begin{array}{l}17.98 \\
17.74 \\
9.66\end{array}$ & $\begin{array}{l}73.24 \\
68.15 \\
70.82 \\
\end{array}$ & $\begin{array}{l}4.85 \\
4.08 \\
4.68 \\
\end{array}$ & $\begin{array}{l}505.0 \\
469.9 \\
488.3\end{array}$ & $\begin{array}{l}33.4 \\
28.1 \\
32.3 \\
\end{array}$ \\
\hline $\begin{array}{l}11955 \\
\text { Front } \\
\text { Center } \\
\text { Back }\end{array}$ & $\begin{array}{l}13.36 \\
13.45 \\
13.44\end{array}$ & $\begin{array}{l}0.49 \\
0.27 \\
0.31\end{array}$ & $\begin{array}{l}55.70 \\
55.81 \\
55.77\end{array}$ & $\begin{array}{l}7.14 \\
7.10 \\
7.08\end{array}$ & $\begin{array}{l}22.01 \\
21.96 \\
21.93\end{array}$ & $\begin{array}{l}16.37 \\
15.33 \\
15.87 \\
\end{array}$ & $\begin{array}{l}0.36 \\
0.43 \\
0.44\end{array}$ & $\begin{array}{l}303.44 \\
310.76 \\
318.38\end{array}$ & $\begin{array}{c}19.13 \\
8.83 \\
12.18\end{array}$ & $\begin{array}{l}307.81 \\
319.29 \\
319.78 \\
\end{array}$ & $\begin{array}{l}18.44 \\
12.90 \\
12.51 \\
\end{array}$ & \begin{tabular}{|l|}
71.70 \\
71.32 \\
72.45 \\
\end{tabular} & $\begin{array}{l}5.47 \\
5.08 \\
4.15 \\
\end{array}$ & $\begin{array}{l}494.4 \\
491.8 \\
499.5 \\
\end{array}$ & $\begin{array}{l}37.7 \\
35.0 \\
28.6 \\
\end{array}$ \\
\hline $\begin{array}{l}11956 \\
\text { Front } \\
\text { Center } \\
\text { Back }\end{array}$ & $\begin{array}{l}13.24 \\
13.45 \\
13.39\end{array}$ & $\begin{array}{l}0.42 \\
0.48 \\
0.19\end{array}$ & $\begin{array}{l}55.48 \\
55.82 \\
55.66\end{array}$ & $\begin{array}{l}7.10 \\
7.05 \\
7.02\end{array}$ & $\begin{array}{l}21.92 \\
21.96 \\
21.86\end{array}$ & $\begin{array}{l}17.63 \\
17.40 \\
17.36\end{array}$ & $\begin{array}{l}0.34 \\
0.38 \\
0.36\end{array}$ & $\begin{array}{l}285.32 \\
301.54 \\
293.95\end{array}$ & $\begin{array}{c}9.77 \\
16.77 \\
12.78\end{array}$ & $\begin{array}{l}294.66 \\
304.13 \\
301.77\end{array}$ & $\begin{array}{c}11.02 \\
9.47 \\
12.40\end{array}$ & $\begin{array}{l}74.18 \\
69.74 \\
72.63 \\
\end{array}$ & $\begin{array}{l}5.31 \\
3.87 \\
3.61\end{array}$ & $\begin{array}{l}511.5 \\
480.9 \\
500.8\end{array}$ & $\begin{array}{l}36.6 \\
26.7 \\
24.9\end{array}$ \\
\hline
\end{tabular}


Table E3 cont. Average Physical Property Data by Lane.

\begin{tabular}{|c|c|c|c|c|c|c|c|c|c|c|c|c|c|c|c|}
\hline Test Methods & T524 (Direct & onal) & & & & T480 & & $\mathrm{T} 441$ & & & & $T 403$ & & & \\
\hline $\begin{array}{c}\text { Sample } \\
\text { ID } \\
(\#)\end{array}$ & $\begin{array}{l}\text { Conditioned } \\
\text { Color } \\
\text { Brightness }\end{array}$ & $\begin{array}{c}\text { ind Ex } \\
95 \% \\
\text { C.I. }\end{array}$ & $\begin{array}{l}\text { osed to Lig } \\
\mathrm{L}^{*} \\
\text { Lightness }\end{array}$ & $\begin{array}{c}\text { t96 Hours } \\
a^{*} \\
+ \text { Redness } \\
\text {-Greeness }\end{array}$ & $\begin{array}{c}b^{*} \\
+ \text { Yellowness } \\
\text {-Blueness } \\
\end{array}$ & Gloss & $\begin{array}{c}95 \% \\
\text { C.I. }\end{array}$ & $\begin{array}{c}\text { FS Cobb } \\
\text { Sizing } \\
\left(\mathrm{g} / \mathrm{m}^{\wedge} 2\right) \\
\end{array}$ & $\begin{array}{l}95 \% \\
\text { C.I. }\end{array}$ & $\begin{array}{c}\text { WS Cobb } \\
\text { Sizing } \\
\left(\mathrm{g} / \mathrm{m}^{\wedge} 2\right) \\
\end{array}$ & $\begin{array}{c}95 \% \\
\text { C.I. }\end{array}$ & $\begin{array}{l}\text { Burst } \\
\text { (psi) }\end{array}$ & $\begin{array}{l}95 \% \\
\text { C.I. }\end{array}$ & $\begin{array}{l}\text { Burst } \\
(\mathrm{kPa}) \\
\end{array}$ & $\begin{array}{l}95 \% \\
\text { C.I. }\end{array}$ \\
\hline $\begin{array}{c}\text { Commercial } \\
\text { Medium } \\
107811 \\
\text { Center } \\
\text { Edge } \\
\end{array}$ & & & & & & & & $\begin{array}{l}203.07 \\
211.67 \\
\end{array}$ & $\begin{array}{r}10.61 \\
6.14 \\
\end{array}$ & $\begin{array}{r}210.30 \\
212.28 \\
\end{array}$ & $\begin{array}{l}4.28 \\
7.71 \\
\end{array}$ & $\begin{array}{l}35.58 \\
35.34 \\
\end{array}$ & $\begin{array}{l}1.20 \\
1.18 \\
\end{array}$ & $\begin{array}{l}245.3 \\
243.7 \\
\end{array}$ & $\begin{array}{l}8.3 \\
8.2 \\
\end{array}$ \\
\hline $\begin{array}{c}\mathbf{1 0 7 8 1 2} \\
\text { Center } \\
\text { Edge }\end{array}$ & & & & & & & & $\begin{array}{l}225.27 \\
211.32\end{array}$ & $\begin{array}{l}3.50 \\
4.10 \\
\end{array}$ & $\begin{array}{l}224.11 \\
216.87\end{array}$ & $\begin{array}{l}4.51 \\
3.88 \\
\end{array}$ & $\begin{array}{l}34.55 \\
34.74 \\
\end{array}$ & $\begin{array}{l}1.40 \\
1.44\end{array}$ & $\begin{array}{l}238.2 \\
239.5\end{array}$ & $\begin{array}{l}9.7 \\
9.9 \\
\end{array}$ \\
\hline $\begin{array}{c}\mathbf{1 0 7 8 1 3} \\
\text { Center } \\
\text { Edge }\end{array}$ & & & & & & & & $\begin{array}{l}196.02 \\
206.70\end{array}$ & $\begin{array}{c}12.52 \\
6.82 \\
\end{array}$ & $\begin{array}{l}197.27 \\
208.90 \\
\end{array}$ & $\begin{array}{l}14.01 \\
12.62 \\
\end{array}$ & $\begin{array}{l}34.46 \\
35.73 \\
\end{array}$ & $\begin{array}{l}2.66 \\
1.51 \\
\end{array}$ & $\begin{array}{l}237.6 \\
246.3 \\
\end{array}$ & $\begin{array}{l}18.3 \\
10.4 \\
\end{array}$ \\
\hline $\begin{array}{c}107814 \\
\text { Center } \\
\text { Edge }\end{array}$ & . & & & & & & & $\begin{array}{l}227.14 \\
222.71\end{array}$ & $\begin{array}{c}17.86 \\
9.52 \\
\end{array}$ & $\begin{array}{l}252.11 \\
238.97\end{array}$ & $\begin{array}{l}12.00 \\
20.99 \\
\end{array}$ & $\begin{array}{l}34.73 \\
35.36 \\
\end{array}$ & $\begin{array}{l}2.79 \\
1.18 \\
\end{array}$ & $\begin{array}{l}239.4 \\
243.8 \\
\end{array}$ & $\begin{array}{c}19.3 \\
8.1 \\
\end{array}$ \\
\hline $\begin{array}{c}107816 \\
\text { Center } \\
\text { Edge } \\
\end{array}$ & & & & & & & & $\begin{array}{l}242.75 \\
262.33 \\
\end{array}$ & $\begin{array}{l}27.03 \\
14.59 \\
\end{array}$ & $\begin{array}{r}230.50 \\
234.36 \\
\end{array}$ & $\begin{array}{l}12.79 \\
16.88 \\
\end{array}$ & $\begin{array}{l}35.47 \\
35.33 \\
\end{array}$ & $\begin{array}{l}1.26 \\
1.76 \\
\end{array}$ & $\begin{array}{l}244.5 \\
243.6 \\
\end{array}$ & $\begin{array}{c}8.7 \\
12.2 \\
\end{array}$ \\
\hline $\begin{array}{c}\text { Commercial } \\
\text { Liner } \\
\mathbf{2 2 5 1 1 1} \\
\text { Center } \\
\text { Edge }\end{array}$ & $\begin{array}{l}15.90 \\
16.09\end{array}$ & $\begin{array}{l}0.55 \\
0.12\end{array}$ & $\begin{array}{l}59.36 \\
59.32\end{array}$ & $\begin{array}{l}6.39 \\
6.39\end{array}$ & $\begin{array}{l}21.80 \\
21.77\end{array}$ & $\begin{array}{l}6.81 \\
6.74\end{array}$ & $\begin{array}{l}0.19 \\
0.15\end{array}$ & $\begin{array}{l}38.20 \\
39.05\end{array}$ & $\begin{array}{l}2.65 \\
3.25 \\
\end{array}$ & $\begin{array}{l}59.93 \\
53.16\end{array}$ & $\begin{array}{c}10.36 \\
4.50\end{array}$ & $\begin{array}{l}89.82 \\
82.66\end{array}$ & $\begin{array}{l}4.43 \\
4.79\end{array}$ & $\begin{array}{l}619.3 \\
569.9\end{array}$ & $\begin{array}{l}30.5 \\
33.0\end{array}$ \\
\hline $\begin{array}{c}225121 \\
\text { Center } \\
\text { Edge }\end{array}$ & $\begin{array}{l}15.92 \\
15.83\end{array}$ & $\begin{array}{l}0.19 \\
0.35\end{array}$ & $\begin{array}{l}58.95 \\
59.06\end{array}$ & $\begin{array}{l}6.18 \\
6.22\end{array}$ & $\begin{array}{l}21.51 \\
21.60\end{array}$ & $\begin{array}{l}6.84 \\
6.56 \\
\end{array}$ & $\begin{array}{l}0.14 \\
0.21\end{array}$ & $\begin{array}{l}41.79 \\
44.25\end{array}$ & $\begin{array}{l}8.36 \\
4.54\end{array}$ & $\begin{array}{l}61.45 \\
65.14 \\
\end{array}$ & $\begin{array}{c}10.79 \\
4.25 \\
\end{array}$ & $\begin{array}{l}85.02 \\
79.44 \\
\end{array}$ & $\begin{array}{l}4.89 \\
3.11\end{array}$ & $\begin{array}{l}586.2 \\
547.8 \\
\end{array}$ & $\begin{array}{l}33.7 \\
21.5\end{array}$ \\
\hline
\end{tabular}


Table E3 cont. Average Physical Property Data by Lane.

\begin{tabular}{|c|c|c|c|c|c|c|c|c|c|c|c|c|c|c|c|c|}
\hline Test Methods & IPST & & & & T826 & & & & & & & & & & T822 & \\
\hline $\begin{array}{c}\text { Sample } \\
\text { ID } \\
\text { (\#) }\end{array}$ & $\begin{array}{c}\text { MD Crack } \\
\text { Angle } \\
\text { (deg) }\end{array}$ & $\begin{array}{l}95 \% \\
\text { C.1. }\end{array}$ & $\begin{array}{c}\text { CD Crack } \\
\text { Angle } \\
\text { (deg) }\end{array}$ & $\begin{array}{l}95 \% \\
\text { C.I. }\end{array}$ & $\begin{array}{c}\text { CD } \\
\text { STFI } \\
\text { (lbf/in) }\end{array}$ & $\begin{array}{c}95 \% \\
\text { C.I. }\end{array}$ & $\begin{array}{c}\text { MD } \\
\text { STFI } \\
\text { (lbf/in) }\end{array}$ & $\begin{array}{c}95 \% \\
\text { C.I. }\end{array}$ & $\begin{array}{c}\text { CD } \\
\text { STFI Index } \\
\left(\mathrm{N}^{*} \mathrm{~m} / \mathrm{g}\right)\end{array}$ & $\begin{array}{l}95 \% \\
\text { C.I. }\end{array}$ & $\begin{array}{c}\text { MD } \\
\text { STFI Index } \\
\left(N^{*} \mathrm{~m} / \mathrm{g}\right)\end{array}$ & $\begin{array}{c}95 \% \\
\text { C.I. }\end{array}$ & $\begin{array}{c}\text { Geo Mean } \\
\text { STFI Index } \\
\left(\mathrm{N}^{*} \mathrm{~m} / \mathrm{g}\right)\end{array}$ & $\begin{array}{c}95 \% \\
\text { C.I. }\end{array}$ & $\begin{array}{l}\text { CD Ring } \\
\text { Crush } \\
\text { (lbf/in) }\end{array}$ & $\begin{array}{c}95 \% \\
\text { C.I. }\end{array}$ \\
\hline $\begin{array}{c}\mathbf{1 1 9 2 6} \\
\text { Front } \\
\text { Center } \\
\text { Back }\end{array}$ & $\begin{array}{l}61.5 \\
59.8 \\
74.7\end{array}$ & $\begin{array}{l}6.3 \\
2.9 \\
5.9\end{array}$ & $\begin{array}{l}82.8 \\
65.7 \\
65.1\end{array}$ & $\begin{array}{c}5.2 \\
7.7 \\
13.1\end{array}$ & $\begin{array}{l}16.82 \\
15.86 \\
17.48\end{array}$ & $\begin{array}{l}0.75 \\
0.50 \\
0.73\end{array}$ & $\begin{array}{l}28.49 \\
27.19 \\
27.37\end{array}$ & $\begin{array}{l}1.24 \\
0.82 \\
1.11\end{array}$ & $\begin{array}{l}19.63 \\
18.79 \\
20.20\end{array}$ & $\begin{array}{l}0.86 \\
0.60 \\
0.85\end{array}$ & $\begin{array}{l}32.42 \\
32.51 \\
31.47\end{array}$ & $\begin{array}{l}1.46 \\
1.05 \\
1.18\end{array}$ & $\begin{array}{l}25.23 \\
24.70 \\
25.21\end{array}$ & $\begin{array}{l}0.86 \\
0.59 \\
0.75\end{array}$ & $\begin{array}{l}10.36 \\
10.06 \\
10.26\end{array}$ & $\begin{array}{l}0.34 \\
0.19 \\
0.37\end{array}$ \\
\hline $\begin{array}{c}11927 \\
\text { Front } \\
\text { Center } \\
\text { Back }\end{array}$ & $\begin{array}{l}57.0 \\
69.5 \\
67.2\end{array}$ & $\begin{array}{l}3.8 \\
5.1 \\
3.1\end{array}$ & $\begin{array}{l}60.4 \\
67.0 \\
70.1\end{array}$ & $\begin{array}{c}5.5 \\
5.1 \\
10.3\end{array}$ & $\begin{array}{l}17.03 \\
15.89 \\
16.39\end{array}$ & $\begin{array}{l}0.57 \\
0.53 \\
0.69\end{array}$ & $\begin{array}{l}28.91 \\
28.62 \\
27.75\end{array}$ & $\begin{array}{l}1.19 \\
0.76 \\
0.71\end{array}$ & $\begin{array}{l}19.94 \\
18.67 \\
19.11\end{array}$ & $\begin{array}{l}0.66 \\
0.61 \\
0.83\end{array}$ & $\begin{array}{l}34.19 \\
33.46 \\
33.26\end{array}$ & $\begin{array}{l}1.41 \\
0.86 \\
0.87\end{array}$ & $\begin{array}{l}26.11 \\
25.00 \\
25.21\end{array}$ & $\begin{array}{l}0.76 \\
0.59 \\
0.67\end{array}$ & $\begin{array}{c}10.70 \\
9.95 \\
10.20\end{array}$ & $\begin{array}{l}0.53 \\
0.23 \\
0.58\end{array}$ \\
\hline $\begin{array}{l}11928 \\
\text { Front } \\
\text { Center } \\
\text { Back }\end{array}$ & $\begin{array}{l}65.2 \\
73.3 \\
66.2 \\
\end{array}$ & $\begin{array}{l}4.0 \\
3.3 \\
7.3 \\
\end{array}$ & $\begin{array}{l}65.7 \\
72.3 \\
58.0 \\
\end{array}$ & $\begin{array}{c}5.8 \\
5.2 \\
10.4 \\
\end{array}$ & $\begin{array}{l}17.26 \\
16.19 \\
16.16 \\
\end{array}$ & $\begin{array}{l}0.76 \\
0.65 \\
0.75\end{array}$ & $\begin{array}{l}29.58 \\
29.39 \\
29.01 \\
\end{array}$ & $\begin{array}{l}1.07 \\
0.76 \\
1.11\end{array}$ & $\begin{array}{l}20.27 \\
18.84 \\
18.92 \\
\end{array}$ & $\begin{array}{l}0.89 \\
0.74 \\
0.90\end{array}$ & $\begin{array}{l}34.03 \\
34.07 \\
33.87\end{array}$ & $\begin{array}{l}1.22 \\
0.89 \\
1.30\end{array}$ & $\begin{array}{l}26.26 \\
25.33 \\
25.29 \\
\end{array}$ & $\begin{array}{l}0.82 \\
0.67 \\
0.74\end{array}$ & $\begin{array}{c}10.36 \\
9.86 \\
10.31 \\
\end{array}$ & $\begin{array}{l}0.38 \\
0.24 \\
0.25 \\
\end{array}$ \\
\hline $\begin{array}{c}11930 \\
\text { Front } \\
\text { Center } \\
\text { Back }\end{array}$ & $\begin{array}{l}70.4 \\
70.0 \\
69.0\end{array}$ & $\begin{array}{l}4.9 \\
5.5 \\
4.6\end{array}$ & $\begin{array}{l}76.2 \\
69.7 \\
70.4\end{array}$ & $\begin{array}{c}10.4 \\
3.5 \\
3.8\end{array}$ & $\begin{array}{l}17.53 \\
15.84 \\
17.03\end{array}$ & $\begin{array}{l}0.78 \\
0.51 \\
0.48\end{array}$ & $\begin{array}{l}30.11 \\
29.92 \\
29.42\end{array}$ & $\begin{array}{l}1.17 \\
0.80 \\
0.97\end{array}$ & $\begin{array}{l}19.86 \\
18.11 \\
19.85\end{array}$ & $\begin{array}{l}0.89 \\
0.59 \\
0.60\end{array}$ & $\begin{array}{l}34.29 \\
34.06 \\
33.21 \\
\end{array}$ & $\begin{array}{l}1.32 \\
0.87 \\
1.06\end{array}$ & $\begin{array}{l}26.09 \\
24.83 \\
25.67\end{array}$ & $\begin{array}{l}0.83 \\
0.56 \\
0.56\end{array}$ & $\begin{array}{l}10.60 \\
10.15 \\
10.83 \\
\end{array}$ & $\begin{array}{l}0.28 \\
0.22 \\
0.35\end{array}$ \\
\hline $\begin{array}{c}11931 \\
\text { Front } \\
\text { Center } \\
\text { Back }\end{array}$ & $\begin{array}{l}60.1 \\
59.7 \\
74.1 \\
\end{array}$ & $\begin{array}{l}5.4 \\
7.7 \\
8.9\end{array}$ & $\begin{array}{l}70.5 \\
63.2 \\
64.5 \\
\end{array}$ & $\begin{array}{l}5.1 \\
4.9 \\
3.3\end{array}$ & $\begin{array}{l}17.31 \\
16.09 \\
16.85\end{array}$ & $\begin{array}{l}0.57 \\
0.66 \\
0.62 \\
\end{array}$ & $\begin{array}{l}29.50 \\
29.75 \\
29.85\end{array}$ & $\begin{array}{l}0.91 \\
0.80 \\
0.97\end{array}$ & $\begin{array}{l}20.59 \\
18.95 \\
19.84\end{array}$ & $\begin{array}{l}0.68 \\
0.76 \\
0.72\end{array}$ & $\begin{array}{l}35.30 \\
34.76 \\
34.18 \\
\end{array}$ & $\begin{array}{l}1.09 \\
0.89 \\
1.09\end{array}$ & $\begin{array}{l}26.96 \\
25.66 \\
26.04 \\
\end{array}$ & $\begin{array}{l}0.65 \\
0.68 \\
0.68\end{array}$ & $\begin{array}{l}10.24 \\
10.01 \\
10.03 \\
\end{array}$ & $\begin{array}{l}0.72 \\
0.24 \\
0.61 \\
\end{array}$ \\
\hline $\begin{array}{l}11932 \\
\text { Front } \\
\text { Center } \\
\text { Back }\end{array}$ & $\begin{array}{l}72.1 \\
70.3 \\
73.2 \\
\end{array}$ & $\begin{array}{l}3.1 \\
4.8 \\
7.0\end{array}$ & $\begin{array}{l}68.4 \\
66.5 \\
73.9 \\
\end{array}$ & $\begin{array}{l}9.8 \\
6.6 \\
6.4 \\
\end{array}$ & $\begin{array}{l}16.97 \\
15.76 \\
16.48 \\
\end{array}$ & $\begin{array}{l}0.58 \\
0.64 \\
0.52\end{array}$ & $\begin{array}{r}29.74 \\
28.49 \\
27.56 \\
\end{array}$ & $\begin{array}{l}0.96 \\
0.77 \\
1.12\end{array}$ & $\begin{array}{l}20.04 \\
18.62 \\
19.49\end{array}$ & $\begin{array}{l}0.67 \\
0.75 \\
0.62\end{array}$ & $\begin{array}{l}35.34 \\
33.34 \\
31.77\end{array}$ & $\begin{array}{l}1.14 \\
0.84 \\
1.06\end{array}$ & $\begin{array}{l}26.61 \\
24.91 \\
24.89 \\
\end{array}$ & $\begin{array}{l}0.68 \\
0.67 \\
0.55 \\
\end{array}$ & $\begin{array}{c}10.33 \\
9.80 \\
10.13\end{array}$ & $\begin{array}{l}0.52 \\
0.21 \\
0.43 \\
\end{array}$ \\
\hline $\begin{array}{l}11935 \\
\text { Front } \\
\text { Center } \\
\text { Back }\end{array}$ & $\begin{array}{l}54.9 \\
56.4 \\
62.7\end{array}$ & $\begin{array}{l}5.7 \\
3.5 \\
3.6\end{array}$ & $\begin{array}{l}65.0 \\
73.2 \\
72.7\end{array}$ & $\begin{array}{l}6.2 \\
4.7 \\
7.1\end{array}$ & $\begin{array}{l}17.50 \\
15.35 \\
16.43\end{array}$ & $\begin{array}{l}0.74 \\
0.53 \\
0.77\end{array}$ & $\begin{array}{l}28.93 \\
28.28 \\
27.00\end{array}$ & $\begin{array}{l}0.74 \\
0.79 \\
0.93\end{array}$ & $\begin{array}{l}20.52 \\
18.17 \\
19.72\end{array}$ & $\begin{array}{l}0.91 \\
0.63 \\
0.90\end{array}$ & $\begin{array}{l}34.68 \\
33.89 \\
32.61\end{array}$ & $\begin{array}{l}0.87 \\
0.91 \\
1.13\end{array}$ & $\begin{array}{l}26.68 \\
24.82 \\
25.36\end{array}$ & $\begin{array}{l}0.72 \\
0.61 \\
0.80\end{array}$ & $\begin{array}{c}10.52 \\
9.75 \\
10.04\end{array}$ & $\begin{array}{l}0.41 \\
0.28 \\
0.20\end{array}$ \\
\hline $\begin{array}{c}11934 \\
\text { Front } \\
\text { Center } \\
\text { Back }\end{array}$ & $\begin{array}{l}64.8 \\
68.7 \\
74.4\end{array}$ & $\begin{array}{l}4.8 \\
5.0 \\
5.2\end{array}$ & $\begin{array}{l}64.4 \\
68.7 \\
74.8\end{array}$ & $\begin{array}{l}6.9 \\
6.3 \\
8.2\end{array}$ & $\begin{array}{l}17.23 \\
16.01 \\
16.92\end{array}$ & $\begin{array}{l}0.52 \\
0.61 \\
0.61\end{array}$ & $\begin{array}{l}29.65 \\
29.47 \\
28.31\end{array}$ & $\begin{array}{l}0.95 \\
0.82 \\
1.06\end{array}$ & $\begin{array}{l}20.19 \\
18.68 \\
19.66\end{array}$ & $\begin{array}{l}0.60 \\
0.71 \\
0.70\end{array}$ & $\begin{array}{l}34.52 \\
34.27 \\
32.95\end{array}$ & $\begin{array}{l}1.10 \\
0.98 \\
1.27\end{array}$ & $\begin{array}{l}26.40 \\
25.31 \\
25.45\end{array}$ & $\begin{array}{l}0.63 \\
0.67 \\
0.72\end{array}$ & $\begin{array}{c}10.40 \\
9.92 \\
10.23\end{array}$ & $\begin{array}{l}0.46 \\
0.37 \\
0.36\end{array}$ \\
\hline
\end{tabular}


Table E3 cont. Average Physical Property Data by Lane.

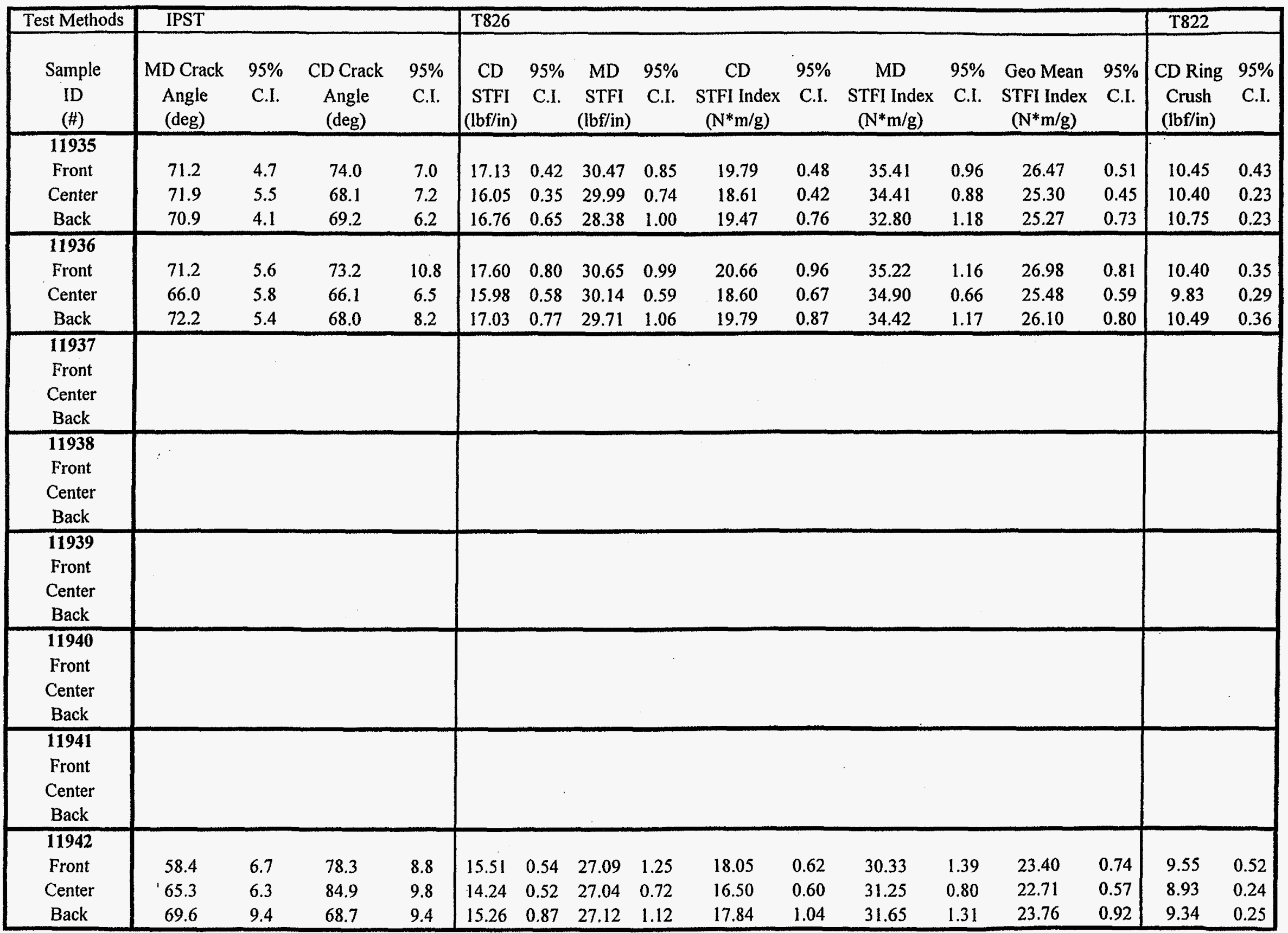


Table E3 cont. Average Physical Property Data by Lane.

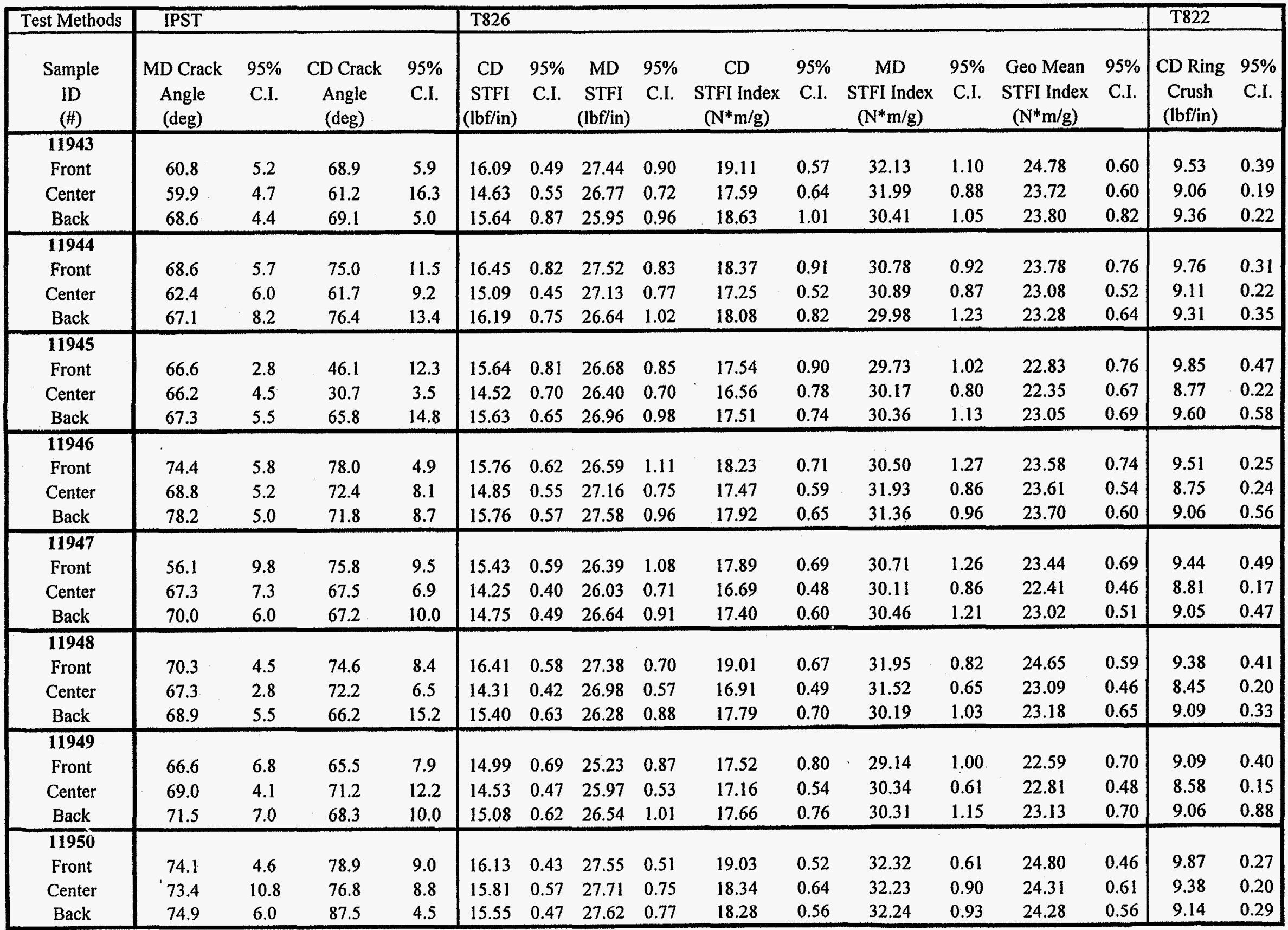


Table E3 cont. Average Physical Property Data by Lane.

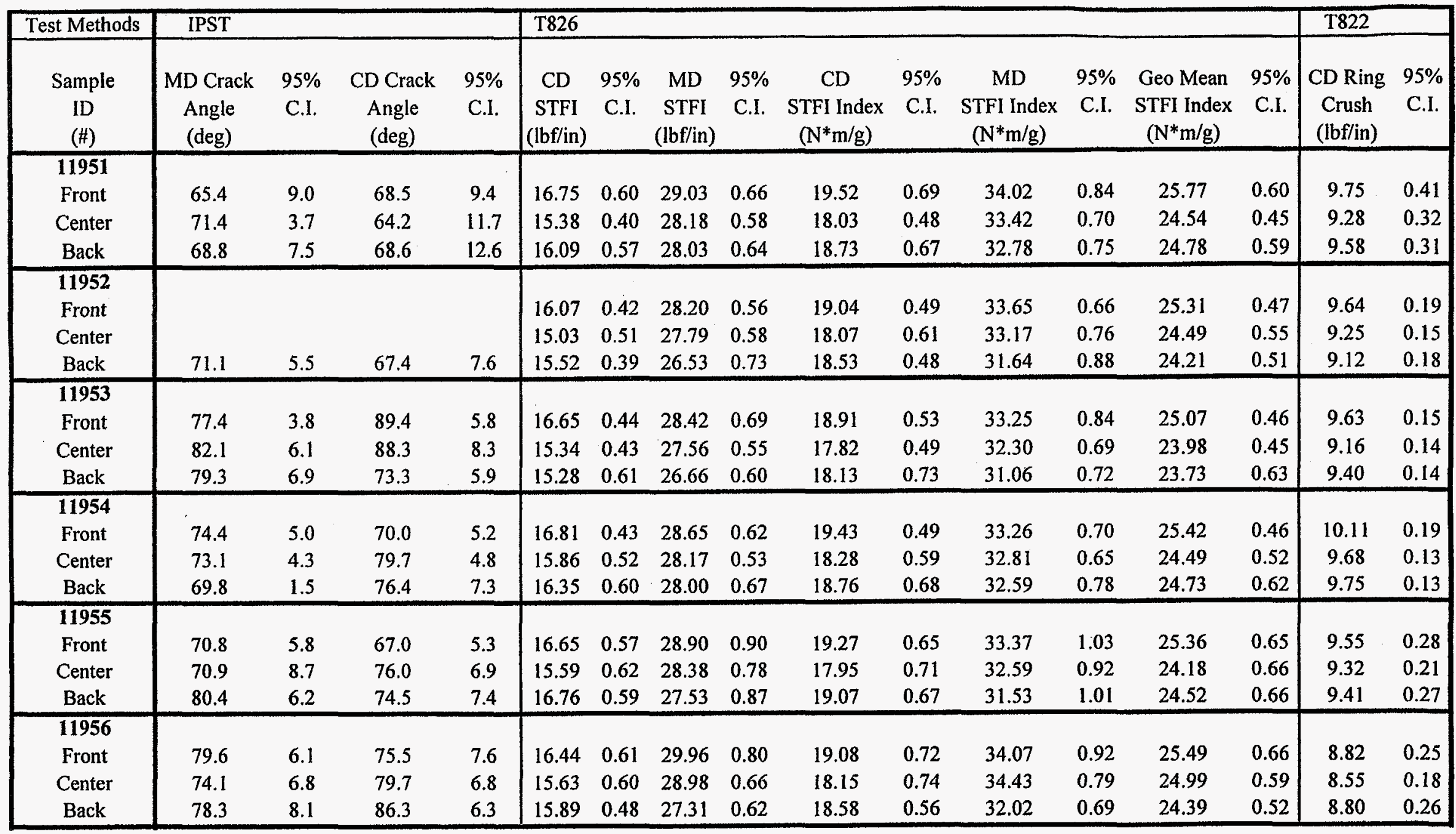


Table E3 cont. Average Physical Property Data by Lane.

\begin{tabular}{|c|c|c|c|c|c|c|c|c|c|c|c|c|c|c|c|c|}
\hline Test Methods & IPST & & & & T826 & & & & & & & & & & $\mathrm{T} 822$ & \\
\hline $\begin{array}{c}\text { Sample } \\
\text { ID } \\
(\#) \\
\end{array}$ & $\begin{array}{c}\text { MD Crack } \\
\text { Angle } \\
\text { (deg) } \\
\end{array}$ & $\begin{array}{l}95 \% \\
\text { C.I. }\end{array}$ & $\begin{array}{c}\text { CD Crack } \\
\text { Angle } \\
\text { (deg) } \\
\end{array}$ & $\begin{array}{l}95 \% \\
\text { C.I. }\end{array}$ & $\begin{array}{c}\mathrm{CD} \\
\text { STFI } \\
(\mathrm{lbf} / \mathrm{in}) \\
\end{array}$ & $\begin{array}{l}95 \% \\
\text { C.I. }\end{array}$ & $\begin{array}{c}\mathrm{MD} \\
\text { STFI } \\
\text { (lbf/in) } \\
\end{array}$ & $\begin{array}{l}95 \% \\
\text { C.I. }\end{array}$ & $\begin{array}{c}\text { CD } \\
\text { STFI Index } \\
\left(\mathrm{N}^{*} \mathrm{~m} / \mathrm{g}\right)\end{array}$ & $\begin{array}{l}95 \% \\
\text { C.I. }\end{array}$ & $\begin{array}{c}\text { MD } \\
\text { STFI Index } \\
\left(\mathrm{N}^{*} \mathrm{~m} / \mathrm{g}\right)\end{array}$ & $\begin{array}{l}95 \% \\
\text { C.I. }\end{array}$ & $\begin{array}{l}\text { Geo Mean } \\
\text { STFI Index } \\
\left(\mathrm{N}^{*} \mathrm{~m} / \mathrm{g}\right)\end{array}$ & $\begin{array}{l}95 \% \\
\text { C.I. }\end{array}$ & $\begin{array}{c}\text { CD Ring } \\
\text { Crush } \\
\text { (lbf/in) }\end{array}$ & $\begin{array}{c}95 \% \\
\text { C.I. }\end{array}$ \\
\hline $\begin{array}{c}\text { Commercial } \\
\text { Medium } \\
107811 \\
\text { Center } \\
\text { Edge } \\
\end{array}$ & & & & & $\begin{array}{l}11.69 \\
10.75 \\
\end{array}$ & $\begin{array}{l}0.43 \\
0.25 \\
\end{array}$ & $\begin{array}{l}21.35 \\
21.29 \\
\end{array}$ & $\begin{array}{l}0.60 \\
0.79 \\
\end{array}$ & $\begin{array}{l}17.33 \\
16.18\end{array}$ & $\begin{array}{l}0.60 \\
0.38\end{array}$ & $\begin{array}{l}32.14 \\
31.31\end{array}$ & $\begin{array}{l}0.85 \\
1.07\end{array}$ & $\begin{array}{l}23.60 \\
22.50\end{array}$ & $\begin{array}{l}0.57 \\
0.51\end{array}$ & $\begin{array}{l}5.17 \\
5.37 \\
\end{array}$ & $\begin{array}{l}0.19 \\
0.15\end{array}$ \\
\hline $\begin{array}{c}\mathbf{1 0 7 8 1 2} \\
\text { Center } \\
\text { Edge }\end{array}$ & & & & & $\begin{array}{l}10.77 \\
11.03\end{array}$ & $\begin{array}{l}0.61 \\
0.23\end{array}$ & $\begin{array}{l}20.19 \\
19.93\end{array}$ & $\begin{array}{l}0.64 \\
0.34\end{array}$ & $\begin{array}{l}16.33 \\
16.93 \\
\end{array}$ & $\begin{array}{l}0.90 \\
0.32\end{array}$ & $\begin{array}{l}30.74 \\
30.85 \\
\end{array}$ & $\begin{array}{l}0.98 \\
0.42 \\
\end{array}$ & $\begin{array}{l}22.39 \\
22.85\end{array}$ & $\begin{array}{l}0.76 \\
0.29 \\
\end{array}$ & $\begin{array}{l}5.13 \\
5.05 \\
\end{array}$ & $\begin{array}{l}0.33 \\
0.21 \\
\end{array}$ \\
\hline $\begin{array}{c}\mathbf{1 0 7 8 1 3} \\
\text { Center } \\
\text { Edge }\end{array}$ & & & & & $\begin{array}{l}10.35 \\
10.91 \\
\end{array}$ & $\begin{array}{l}0.44 \\
0.22\end{array}$ & $\begin{array}{l}20.00 \\
20.70\end{array}$ & $\begin{array}{l}0.51 \\
0.40\end{array}$ & $\begin{array}{l}16.02 \\
16.65\end{array}$ & $\begin{array}{l}0.66 \\
0.33\end{array}$ & $\begin{array}{l}31.10 \\
31.60\end{array}$ & $\begin{array}{l}0.79 \\
0.62\end{array}$ & $\begin{array}{l}22.32 \\
22.94\end{array}$ & $\begin{array}{l}0.56 \\
0.36\end{array}$ & $\begin{array}{l}4.53 \\
4.83 \\
\end{array}$ & $\begin{array}{l}0.28 \\
0.21\end{array}$ \\
\hline $\begin{array}{c}\mathbf{1 0 7 8 1 4} \\
\text { Center } \\
\text { Edge }\end{array}$ & & & & & $\begin{array}{l}11.14 \\
10.97\end{array}$ & $\begin{array}{l}0.29 \\
0.23\end{array}$ & $\begin{array}{l}21.99 \\
20.24\end{array}$ & $\begin{array}{l}0.49 \\
0.57\end{array}$ & $\begin{array}{l}16.86 \\
16.39\end{array}$ & $\begin{array}{l}0.44 \\
0.34\end{array}$ & $\begin{array}{l}31.66 \\
30.35\end{array}$ & $\begin{array}{l}0.69 \\
0.73\end{array}$ & $\begin{array}{l}23.10 \\
22.29\end{array}$ & $\begin{array}{l}0.41 \\
0.32\end{array}$ & $\begin{array}{l}5.35 \\
5.12\end{array}$ & $\begin{array}{l}0.28 \\
0.16\end{array}$ \\
\hline $\begin{array}{c}107816 \\
\text { Center } \\
\text { Edge } \\
\end{array}$ & & & & & $\begin{array}{l}10.57 \\
11.09 \\
\end{array}$ & $\begin{array}{l}0.26 \\
0.21 \\
\end{array}$ & $\begin{array}{l}20.58 \\
21.47 \\
\end{array}$ & $\begin{array}{l}0.53 \\
0.46 \\
\end{array}$ & $\begin{array}{l}16.56 \\
16.64 \\
\end{array}$ & $\begin{array}{l}0.37 \\
0.29 \\
\end{array}$ & $\begin{array}{l}31.29 \\
32.28 \\
\end{array}$ & $\begin{array}{l}0.83 \\
0.63 \\
\end{array}$ & $\begin{array}{l}22.76 \\
23.17 \\
\end{array}$ & $\begin{array}{l}0.42 \\
0.30 \\
\end{array}$ & $\begin{array}{l}4.86 \\
4.97 \\
\end{array}$ & $\begin{array}{l}0.35 \\
0.15 \\
\end{array}$ \\
\hline $\begin{array}{c}\text { Commercial } \\
\text { Liner } \\
225111 \\
\text { Center } \\
\text { Edge } \\
\end{array}$ & $\begin{array}{l}67.1 \\
71.8 \\
\end{array}$ & $\begin{array}{l}4.2 \\
3.6 \\
\end{array}$ & $\begin{array}{l}85.4 \\
83.7 \\
\end{array}$ & $\begin{array}{l}8.9 \\
3.4 \\
\end{array}$ & $\begin{array}{l}17.83 \\
17.91 \\
\end{array}$ & $\begin{array}{l}0.69 \\
0.48 \\
\end{array}$ & $\begin{array}{l}31.04 \\
33.80 \\
\end{array}$ & $\begin{array}{l}0.72 \\
0.77 \\
\end{array}$ & $\begin{array}{l}21.33 \\
21.71 \\
\end{array}$ & $\begin{array}{l}0.82 \\
0.56 \\
\end{array}$ & $\begin{array}{l}37.06 \\
40.30 \\
\end{array}$ & $\begin{array}{l}0.90 \\
0.88 \\
\end{array}$ & $\begin{array}{l}28.12 \\
29.58 \\
\end{array}$ & $\begin{array}{l}0.70 \\
0.54 \\
\end{array}$ & $\begin{array}{c}10.51 \\
9.90 \\
\end{array}$ & $\begin{array}{l}0.45 \\
0.41 \\
\end{array}$ \\
\hline $\begin{array}{c}\mathbf{2 2 5 1 2 1} \\
\text { Center } \\
\text { Edge }\end{array}$ & $\begin{array}{l}77.8 \\
70.3\end{array}$ & $\begin{array}{l}3.6 \\
3.6\end{array}$ & $\begin{array}{l}89.5 \\
81.2\end{array}$ & $\begin{array}{l}7.2 \\
5.5\end{array}$ & $\begin{array}{l}17.97 \\
18.20\end{array}$ & $\begin{array}{l}0.81 \\
0.50\end{array}$ & $\begin{array}{l}32.27 \\
33.65\end{array}$ & $\begin{array}{l}1.18 \\
0.63\end{array}$ & $\begin{array}{l}21.23 \\
21.75\end{array}$ & $\begin{array}{l}0.98 \\
0.62\end{array}$ & $\begin{array}{l}38.31 \\
40.48\end{array}$ & $\begin{array}{l}1.36 \\
0.73\end{array}$ & $\begin{array}{l}28.51 \\
29.67 \\
\end{array}$ & $\begin{array}{l}0.90 \\
0.56\end{array}$ & $\begin{array}{l}10.57 \\
10.42 \\
\end{array}$ & $\begin{array}{l}0.45 \\
0.42\end{array}$ \\
\hline
\end{tabular}


Table E3 cont. Average Physical Property Data by Lane.

\begin{tabular}{|c|c|c|c|c|c|c|c|c|c|c|c|c|c|c|}
\hline Test Methods & & & & & & & T494 & & & & & & & \\
\hline $\begin{array}{l}\text { Sample } \\
\text { ID } \\
(\#)\end{array}$ & $\begin{array}{c}\text { MD Ring } \\
\text { Crush } \\
\text { (lbf/in) }\end{array}$ & $\begin{array}{l}95 \% \\
\text { C.I. }\end{array}$ & $\begin{array}{l}\text { CD R. C. } \\
\text { Index } \\
\left(N^{*} m / g\right)\end{array}$ & $\begin{array}{l}95 \% \\
\text { C.I. }\end{array}$ & $\begin{array}{c}\text { MD R. C. } \\
\text { Index } \\
\left(N^{*} \mathrm{~m} / \mathrm{g}\right)\end{array}$ & $\begin{array}{l}95 \% \\
\text { C.I. }\end{array}$ & $\begin{array}{c}\text { CD } \\
\text { Tensile } \\
\text { (lbf/in) }\end{array}$ & $\begin{array}{l}95 \% \\
\text { C.I. }\end{array}$ & $\begin{array}{c}\text { MD } \\
\text { Tensile } \\
\text { (lbf/in) }\end{array}$ & $\begin{array}{l}95 \% \\
\text { C.I. }\end{array}$ & $\begin{array}{c}\mathrm{CD} \\
\text { Tensile } \\
\text { Index } \\
\left(\mathrm{N}^{*} \mathrm{~m} / \mathrm{g}\right)\end{array}$ & $\begin{array}{c}95 \% \\
\text { C.I. }\end{array}$ & $\begin{array}{c}\text { MD } \\
\text { Tensile } \\
\text { Index } \\
\left(\mathrm{N}^{*} \mathrm{~m} / \mathrm{g}\right)\end{array}$ & $\begin{array}{c}95 \% \\
\text { C.I. }\end{array}$ \\
\hline 11926 & & & & & & & & & & & & & & \\
\hline Front & 14.82 & 1.36 & 12.02 & 0.34 & 17.01 & 1.59 & 28.15 & 0.78 & 67.01 & 3.50 & 32.48 & 0.92 & 77.57 & 4.22 \\
\hline Center & 14.66 & 0.28 & 11.85 & 0.19 & 17.43 & 0.36 & 24.50 & 0.85 & 69.03 & 1.40 & 28.88 & 0.96 & 81.52 & 1.51 \\
\hline Back & 14.11 & 1.10 & 11.79 & 0.38 & 16.23 & 1.28 & 27.71 & 0.39 & 66.94 & 1.06 & 32.37 & 0.40 & 78.21 & 1.30 \\
\hline 11927 & & & & & & & & & & & & & & \\
\hline Front & 15.14 & 0.42 & 12.50 & 0.59 & 17.87 & 0.41 & 28.00 & 0.70 & 67.99 & 1.38 & 33.06 & 0.80 & 78.97 & 1.37 \\
\hline Center & 14.81 & 0.18 & 11.61 & 0.27 & 17.33 & 0.25 & 25.08 & 0.53 & 68.84 & 1.58 & 29.37 & 0.55 & 80.81 & 1.83 \\
\hline Back & 14.46 & 0.34 & 11.93 & 0.62 & 16.91 & 0.44 & 27.79 & 0.60 & 64.82 & 2.34 & 32.73 & 0.70 & 76.08 & 2.66 \\
\hline 11928 & & & & & & & & & & & & & & \\
\hline Front & 15.09 & 0.34 & 12.06 & 0.40 & 17.47 & 0.35 & 28.04 & 0.50 & 67.79 & 3.14 & 32.65 & 0.59 & 78.49 & 3.49 \\
\hline Center & 14.39 & 0.27 & 11.44 & 0.23 & 16.73 & 0.30 & 25.50 & 0.36 & 68.80 & 1.81 & 29.50 & 0.43 & 79.68 & 2.06 \\
\hline Back & 14.58 & 0.35 & 11.97 & 0.31 & 16.96 & 0.35 & 28.05 & 0.52 & 64.70 & 2.02 & 32.44 & 0.65 & 75.16 & 1.98 \\
\hline 11930 & & & & & & & & & & & & & & \\
\hline Front & 14.52 & 0.91 & 12.08 & 0.36 & 16.76 & 1.04 & 28.44 & 0.60 & 67.55 & 2.58 & 32.59 & 0.62 & 77.68 & 2.98 \\
\hline Center & 15.11 & 0.25 & 11.63 & 0.25 & 17.43 & 0.27 & 25.30 & 0.67 & 68.78 & 2.68 & 29.18 & 0.79 & 79.10 & 3.45 \\
\hline Back & 15.24 & 0.44 & 12.44 & 0.43 & 17.53 & 0.52 & 28.19 & 1.02 & 66.09 & 1.44 & 32.42 & 1.14 & 75.55 & 1.51 \\
\hline 11931 & & & & & & & & & & & & & & \\
\hline Front & 14.81 & 0.43 & 12.04 & 0.88 & 17.47 & 0.46 & 27.37 & 0.58 & 65.40 & 2.07 & 32.27 & 0.61 & 77.32 & 2.78 \\
\hline Center & 14.42 & 0.22 & 11.74 & 0.28 & 16.93 & 0.29 & 25.53 & 0.29 & 70.81 & 0.87 & 30.00 & 0.54 & 83.48 & 1.10 \\
\hline Back & 14.51 & 0.41 & 11.63 & 0.66 & 17.12 & 0.45 & 28.03 & 0.69 & 66.00 & 1.01 & 33.15 & 0.80 & 78.13 & 1.11 \\
\hline 11932 & & & & & & & & & & & & & & \\
\hline Front & 14.95 & 0.41 & 12.29 & 0.55 & 17.67 & 0.38 & 27.32 & 0.75 & 68.73 & 1.16 & 32.46 & 0.92 & 81.37 & 1.62 \\
\hline Center & 14.26 & 0.37 & 11.61 & 0.23 & 16.92 & 0.44 & 24.76 & 0.40 & 70.24 & 1.15 & 29.44 & 0.45 & 83.33 & 1.02 \\
\hline Back & 14.60 & 0.34 & 12.01 & 0.54 & 17.22 & 0.45 & 28.29 & 0.61 & 65.90 & 2.07 & 33.65 & 0.68 & 78.54 & 1.76 \\
\hline 11933 & & & & & & & & & & & & & & \\
\hline Front & 15.00 & 0.46 & 12.38 & 0.43 & 17.69 & 0.49 & 27.07 & 0.60 & 66.71 & 1.86 & 32.29 & 0.73 & 79.44 & 2.19 \\
\hline Center & 14.16 & 0.41 & 11.58 & 0.34 & 17.03 & 0.47 & 24.06 & 0.33 & 67.54 & 1.25 & 28.55 & 0.50 & 80.74 & 1.59 \\
\hline Back & 14.65 & 0.37 & 11.81 & 0.23 & 17.40 & 0.34 & 27.56 & 0.53 & 65.35 & 3.09 & 32.47 & 0.59 & 78.07 & 3.36 \\
\hline 11934 & & & & & & & & & & & & & & \\
\hline Front & 14.71 & 1.07 & 12.10 & 0.54 & 17.17 & 1.20 & 28.50 & 0.86 & 69.32 & 1.81 & 32.45 & 0.91 & 80.09 & 1.88 \\
\hline Center & 14.63 & 0.30 & 11.49 & 0.38 & 17.15 & 0.36 & 24.41 & 0.55 & 69.94 & 1.48 & 28.68 & 0.57 & 81.71 & 1.84 \\
\hline Back & 14.81 & 0.40 & 11.89 & 0.36 & 17.21 & 0.52 & 28.14 & 0.89 & 66.79 & 2.08 & 32.80 & 0.93 & 77.06 & 1.59 \\
\hline
\end{tabular}


Table E3 cont. Average Physical Property Data by Lane.

\begin{tabular}{|c|c|c|c|c|c|c|c|c|c|c|c|c|c|c|}
\hline Test Methods & & & & & & & T494 & & & & & & & \\
\hline $\begin{array}{l}\text { Sample } \\
\text { ID } \\
\text { (\#) }\end{array}$ & $\begin{array}{c}\text { MD Ring } \\
\text { Crush } \\
\text { (lbf/in) }\end{array}$ & $\begin{array}{c}95 \% \\
\text { C.I. }\end{array}$ & $\begin{array}{c}\text { CD R. C. } \\
\text { Index } \\
\left(N^{*} m / g\right)\end{array}$ & $\begin{array}{c}95 \% \\
\text { C.I. }\end{array}$ & $\begin{array}{l}\text { MD R. C. } \\
\text { Index } \\
\left(\mathrm{N}^{*} \mathrm{~m} / \mathrm{g}\right)\end{array}$ & $\begin{array}{l}95 \% \\
\text { C.I. }\end{array}$ & $\begin{array}{c}\text { CD } \\
\text { Tensile } \\
\text { (lbf/in) }\end{array}$ & $\begin{array}{l}95 \% \\
\text { C.I. }\end{array}$ & $\begin{array}{c}\text { MD } \\
\text { Tensile } \\
\text { (lbf/in) }\end{array}$ & $\begin{array}{c}95 \% \\
\text { C.I. }\end{array}$ & $\begin{array}{c}\mathrm{CD} \\
\text { Tensile } \\
\text { Index } \\
\left(\mathrm{N}^{*} \mathrm{~m} / \mathrm{g}\right)\end{array}$ & $\begin{array}{c}95 \% \\
\text { C.I. }\end{array}$ & $\begin{array}{c}\mathrm{MD} \\
\text { Tensile } \\
\text { Index } \\
\left(\mathrm{N}^{*} \mathrm{~m} / \mathrm{g}\right)\end{array}$ & $\begin{array}{c}95 \% \\
\text { C.I. }\end{array}$ \\
\hline $\begin{array}{l}11935 \\
\text { Front } \\
\text { Center } \\
\text { Back }\end{array}$ & $\begin{array}{l}15.43 \\
14.97 \\
15.05\end{array}$ & $\begin{array}{l}0.55 \\
0.33 \\
0.53\end{array}$ & $\begin{array}{l}12.05 \\
11.93 \\
12.39\end{array}$ & $\begin{array}{l}0.52 \\
0.24 \\
0.27\end{array}$ & $\begin{array}{l}17.81 \\
17.24 \\
17.20\end{array}$ & $\begin{array}{l}0.62 \\
0.38 \\
0.67\end{array}$ & $\begin{array}{l}27.79 \\
25.65 \\
28.54\end{array}$ & $\begin{array}{l}0.52 \\
0.47 \\
0.50\end{array}$ & $\begin{array}{l}68.10 \\
71.47 \\
66.87\end{array}$ & $\begin{array}{l}2.26 \\
0.85 \\
1.83\end{array}$ & $\begin{array}{l}32.15 \\
29.58 \\
32.92\end{array}$ & $\begin{array}{l}0.66 \\
0.52 \\
0.66\end{array}$ & $\begin{array}{l}79.09 \\
82.50 \\
76.57\end{array}$ & $\begin{array}{l}2.61 \\
1.04 \\
1.77\end{array}$ \\
\hline $\begin{array}{c}11936 \\
\text { Front } \\
\text { Center } \\
\text { Back }\end{array}$ & $\begin{array}{l}14.37 \\
14.57 \\
15.07\end{array}$ & $\begin{array}{l}0.36 \\
0.26 \\
0.55\end{array}$ & $\begin{array}{l}12.12 \\
11.57 \\
12.04\end{array}$ & $\begin{array}{l}0.43 \\
0.30 \\
0.38\end{array}$ & $\begin{array}{l}16.77 \\
17.14 \\
17.53\end{array}$ & $\begin{array}{l}0.41 \\
0.28 \\
0.62\end{array}$ & $\begin{array}{l}26.84 \\
24.99 \\
27.50\end{array}$ & $\begin{array}{l}0.97 \\
0.63 \\
0.68\end{array}$ & $\begin{array}{l}68.10 \\
69.33 \\
67.43\end{array}$ & $\begin{array}{l}2.62 \\
1.69 \\
1.52\end{array}$ & $\begin{array}{l}31.41 \\
29.27 \\
32.07\end{array}$ & $\begin{array}{l}1.11 \\
0.74 \\
0.76\end{array}$ & $\begin{array}{l}79.19 \\
80.97 \\
78.80\end{array}$ & $\begin{array}{l}2.98 \\
1.58 \\
1.15\end{array}$ \\
\hline $\begin{array}{l}11937 \\
\text { Front } \\
\text { Center } \\
\text { Back }\end{array}$ & & & & & & & & & & & & & & \\
\hline $\begin{array}{c}11938 \\
\text { Front } \\
\text { Center } \\
\text { Back }\end{array}$ & & & & & & & & & & & & & & \\
\hline $\begin{array}{c}11939 \\
\text { Front } \\
\text { Center } \\
\text { Back }\end{array}$ & & & & & & & & & & & & & & \\
\hline $\begin{array}{l}11940 \\
\text { Front } \\
\text { Center } \\
\text { Back }\end{array}$ & & & & & & & & & & & & & & \\
\hline $\begin{array}{l}\text { 11941 } \\
\text { Front } \\
\text { Center } \\
\text { Back }\end{array}$ & & & & & & & & & & & & & & \\
\hline $\begin{array}{l}11942 \\
\text { Front }\end{array}$ & 12.70 & 0.43 & 10.85 & 0.57 & 14.63 & 0.60 & 24.46 & 0.75 & 59.62 & 2.15 & 27.93 & 0.90 & 67.51 & 2.57 \\
\hline Center & 13.03 & 0.29 & 10.41 & 0.27 & 15.07 & 0.33 & 22.88 & 0.36 & 61.41 & 1.56 & 26.21 & 0.32 & 70.21 & 2.12 \\
\hline Back & 13.33 & 0.46 & 10.86 & 0.31 & 15.43 & 0.51 & 23.91 & 0.74 & 57.87 & 2.13 & 27.77 & 1.01 & 66.51 & 1.98 \\
\hline
\end{tabular}


Table E3 cont. Average Physical Property Data by Lane.

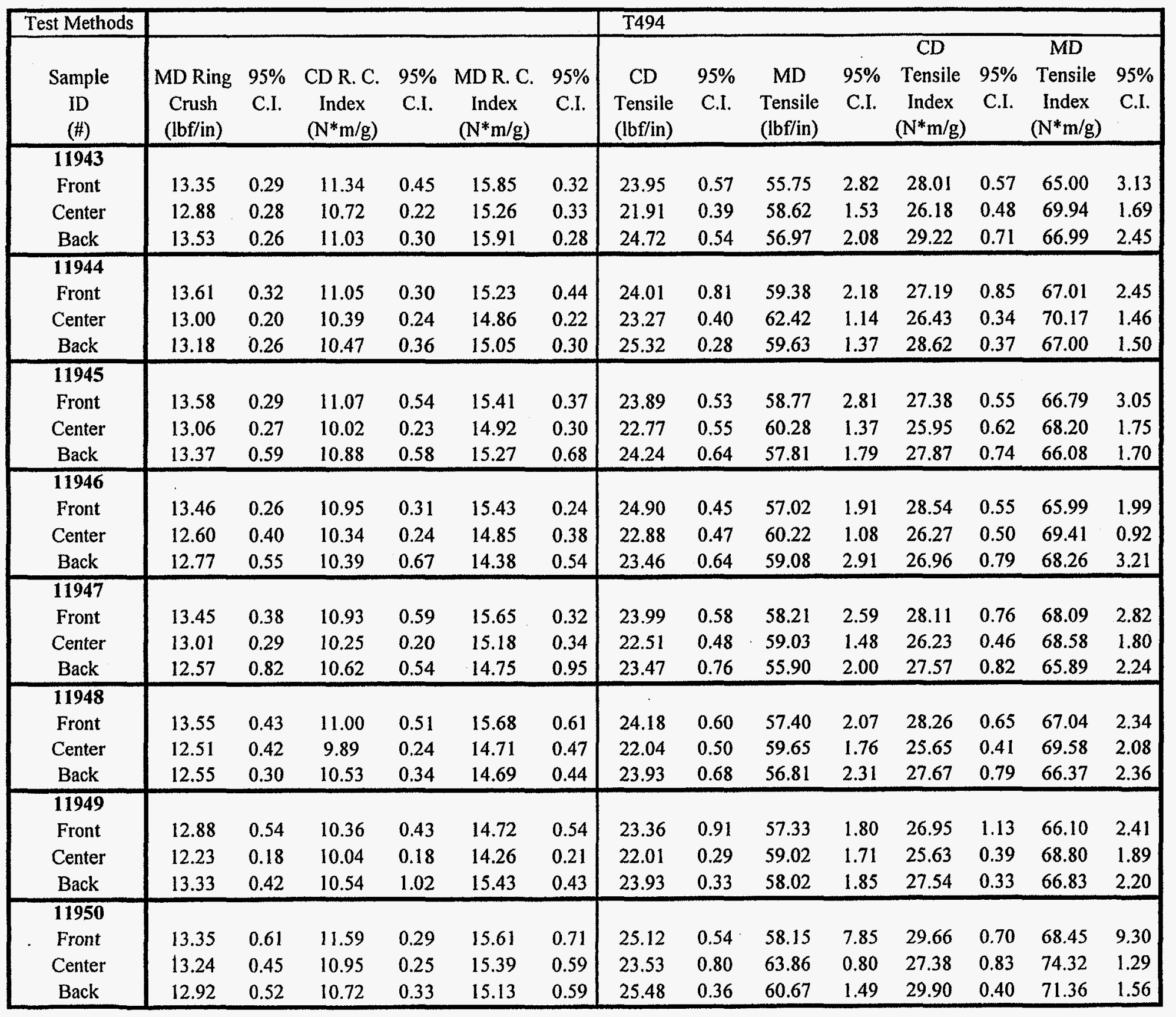


Table E3 cont. Average Physical Property Data by Lane.

\begin{tabular}{|c|c|c|c|c|c|c|c|c|c|c|c|c|c|c|}
\hline Test Methods & & & & & & & T494 & & & & & & & \\
\hline $\begin{array}{l}\text { Sample } \\
\text { ID } \\
(\#)\end{array}$ & $\begin{array}{c}\text { MD Ring } \\
\text { Crush } \\
\text { (lbf/in) }\end{array}$ & $\begin{array}{l}95 \% \\
\text { C.I. }\end{array}$ & $\begin{array}{l}\text { CD R. C. } \\
\text { Index } \\
\left(\mathrm{N}^{*} \mathrm{~m} / \mathrm{g}\right)\end{array}$ & $\begin{array}{c}95 \% \\
\text { C.I. }\end{array}$ & $\begin{array}{l}\text { MD R. C. } \\
\text { Index } \\
\left(N^{*} \mathrm{~m} / \mathrm{g}\right)\end{array}$ & $\begin{array}{c}95 \% \\
\text { C.I. }\end{array}$ & $\begin{array}{c}\text { CD } \\
\text { Tensile } \\
\text { (lbf/in) }\end{array}$ & $\begin{array}{l}95 \% \\
\text { C.I. }\end{array}$ & $\begin{array}{c}\text { MD } \\
\text { Tensile } \\
\text { (lbf/in) }\end{array}$ & $\begin{array}{l}95 \% \\
\text { C.I. }\end{array}$ & $\begin{array}{c}\mathrm{CD} \\
\text { Tensile } \\
\text { Index } \\
\left(\mathrm{N}^{*} \mathrm{~m} / \mathrm{g}\right)\end{array}$ & $\begin{array}{c}95 \% \\
\text { C.I. }\end{array}$ & $\begin{array}{c}\text { MD } \\
\text { Tensile } \\
\text { Index } \\
\left(\mathrm{N}^{*} \mathrm{~m} / \mathrm{g}\right) \\
\end{array}$ & $\begin{array}{c}95 \% \\
\text { C.I. }\end{array}$ \\
\hline $\begin{array}{c}11951 \\
\text { Front } \\
\text { Center } \\
\text { Back }\end{array}$ & $\begin{array}{l}13.18 \\
13.14 \\
13.23\end{array}$ & $\begin{array}{l}0.47 \\
0.51 \\
0.41\end{array}$ & $\begin{array}{l}11.42 \\
10.88 \\
11.27\end{array}$ & $\begin{array}{l}0.47 \\
0.35 \\
0.35\end{array}$ & $\begin{array}{l}15.43 \\
15.40 \\
15.43\end{array}$ & $\begin{array}{l}0.54 \\
0.60 \\
0.50\end{array}$ & $\begin{array}{l}25.91 \\
23.34 \\
25.83\end{array}$ & $\begin{array}{l}0.47 \\
0.69 \\
0.45\end{array}$ & $\begin{array}{l}60.55 \\
64.18 \\
60.02\end{array}$ & $\begin{array}{l}1.81 \\
0.99 \\
1.90\end{array}$ & $\begin{array}{l}30.14 \\
27.43 \\
30.46 \\
\end{array}$ & $\begin{array}{l}0.64 \\
0.75 \\
0.48\end{array}$ & $\begin{array}{l}70.94 \\
74.89 \\
70.25 \\
\end{array}$ & $\begin{array}{l}2.27 \\
1.36 \\
1.90 \\
\end{array}$ \\
\hline $\begin{array}{l}11952 \\
\text { Front } \\
\text { Center } \\
\text { Back }\end{array}$ & $\begin{array}{l}13.15 \\
13.02 \\
12.66\end{array}$ & $\begin{array}{l}0.27 \\
0.24 \\
0.25\end{array}$ & $\begin{array}{l}11.46 \\
11.01 \\
10.88\end{array}$ & $\begin{array}{l}0.20 \\
0.16 \\
0.19\end{array}$ & $\begin{array}{l}15.62 \\
15.47 \\
15.12\end{array}$ & $\begin{array}{l}0.29 \\
0.26 \\
0.30\end{array}$ & $\begin{array}{l}25.33 \\
23.43 \\
25.21\end{array}$ & $\begin{array}{l}0.78 \\
0.38 \\
0.65\end{array}$ & $\begin{array}{l}62.85 \\
63.63 \\
59.78\end{array}$ & $\begin{array}{l}2.10 \\
1.22 \\
1.35\end{array}$ & $\begin{array}{l}30.06 \\
27.72 \\
29.94\end{array}$ & $\begin{array}{l}0.91 \\
0.50 \\
0.73\end{array}$ & $\begin{array}{l}75.09 \\
75.64 \\
71.06\end{array}$ & $\begin{array}{l}2.23 \\
1.55 \\
1.80\end{array}$ \\
\hline $\begin{array}{l}11953 \\
\text { Front } \\
\text { Center } \\
\text { Back }\end{array}$ & $\begin{array}{l}13.49 \\
13.29 \\
13.09\end{array}$ & $\begin{array}{l}0.21 \\
0.15 \\
0.17\end{array}$ & $\begin{array}{l}11.32 \\
10.75 \\
11.04\end{array}$ & $\begin{array}{l}0.16 \\
0.14 \\
0.16\end{array}$ & $\begin{array}{l}15.87 \\
15.51 \\
15.53\end{array}$ & $\begin{array}{l}0.23 \\
0.17 \\
0.20\end{array}$ & $\begin{array}{l}25.60 \\
23.77 \\
25.48 \\
\end{array}$ & $\begin{array}{l}0.83 \\
0.53 \\
0.70\end{array}$ & $\begin{array}{l}63.43 \\
62.62 \\
60.21 \\
\end{array}$ & $\begin{array}{l}1.99 \\
1.69 \\
1.34 \\
\end{array}$ & $\begin{array}{l}30.16 \\
27.89 \\
30.43\end{array}$ & $\begin{array}{l}0.80 \\
0.64 \\
0.66\end{array}$ & $\begin{array}{l}75.31 \\
74.46 \\
71.84\end{array}$ & $\begin{array}{l}1.98 \\
2.09 \\
1.68\end{array}$ \\
\hline $\begin{array}{l}11954 \\
\text { Front } \\
\text { Center } \\
\text { Back }\end{array}$ & $\begin{array}{l}13.77 \\
13.56 \\
13.40\end{array}$ & $\begin{array}{l}0.18 \\
0.18 \\
0.22\end{array}$ & $\begin{array}{l}11.63 \\
11.16 \\
11.22\end{array}$ & $\begin{array}{l}0.22 \\
0.15 \\
0.15\end{array}$ & $\begin{array}{l}15.93 \\
15.63 \\
15.54\end{array}$ & $\begin{array}{l}0.20 \\
0.20 \\
0.22\end{array}$ & $\begin{array}{l}26.14 \\
23.95 \\
26.74\end{array}$ & $\begin{array}{l}0.66 \\
0.44 \\
0.62\end{array}$ & $\begin{array}{l}65.49 \\
66.21 \\
59.96\end{array}$ & $\begin{array}{l}2.08 \\
1.61 \\
1.76\end{array}$ & $\begin{array}{l}30.23 \\
27.87 \\
31.09\end{array}$ & $\begin{array}{l}0.73 \\
0.49 \\
0.60\end{array}$ & $\begin{array}{l}75.83 \\
76.56 \\
69.83\end{array}$ & $\begin{array}{l}2.49 \\
1.57 \\
2.37\end{array}$ \\
\hline $\begin{array}{l}11955 \\
\text { Front } \\
\text { Center } \\
\text { Back }\end{array}$ & $\begin{array}{l}12.57 \\
12.73 \\
12.75\end{array}$ & $\begin{array}{l}0.26 \\
0.30 \\
0.32\end{array}$ & $\begin{array}{l}10.98 \\
10.65 \\
10.78\end{array}$ & $\begin{array}{l}0.31 \\
0.21 \\
0.31\end{array}$ & $\begin{array}{l}14.54 \\
14.64 \\
14.67\end{array}$ & $\begin{array}{l}0.27 \\
0.31 \\
0.32\end{array}$ & $\begin{array}{l}26.33 \\
23.98 \\
26.77\end{array}$ & $\begin{array}{l}0.73 \\
0.26 \\
0.58\end{array}$ & $\begin{array}{l}63.04 \\
65.65 \\
62.74\end{array}$ & $\begin{array}{l}2.42 \\
1.00 \\
1.04\end{array}$ & $\begin{array}{l}30.38 \\
27.68 \\
30.65 \\
\end{array}$ & $\begin{array}{l}0.93 \\
0.39 \\
0.62\end{array}$ & $\begin{array}{l}72.97 \\
75.86 \\
72.10\end{array}$ & $\begin{array}{l}2.89 \\
1.34 \\
1.41 \\
\end{array}$ \\
\hline $\begin{array}{c}11956 \\
\text { Front } \\
\text { Center } \\
\text { Back }\end{array}$ & $\begin{array}{l}12.16 \\
11.97 \\
12.05\end{array}$ & $\begin{array}{l}0.24 \\
0.28 \\
0.33\end{array}$ & $\begin{array}{c}10.14 \\
9.94 \\
10.25\end{array}$ & $\begin{array}{l}0.26 \\
0.19 \\
0.30\end{array}$ & $\begin{array}{l}13.96 \\
13.82 \\
13.99\end{array}$ & $\begin{array}{l}0.25 \\
0.29 \\
0.40\end{array}$ & $\begin{array}{l}26.26 \\
24.63 \\
26.81\end{array}$ & $\begin{array}{l}0.62 \\
0.61 \\
0.48\end{array}$ & $\begin{array}{l}65.02 \\
64.11 \\
63.10 \\
\end{array}$ & $\begin{array}{l}2.78 \\
2.33 \\
1.62 \\
\end{array}$ & $\begin{array}{l}30.61 \\
29.03 \\
31.45 \\
\end{array}$ & $\begin{array}{l}0.73 \\
0.66 \\
0.58\end{array}$ & $\begin{array}{l}75.51 \\
74.89 \\
73.46 \\
\end{array}$ & $\begin{array}{l}2.93 \\
2.61 \\
1.55 \\
\end{array}$ \\
\hline
\end{tabular}


Table E3 cont. Average Physical Property Data by Lane.

\begin{tabular}{|c|c|c|c|c|c|c|c|c|c|c|c|c|c|c|}
\hline Test Methods & & & & & & & T494 & & & & & & & \\
\hline $\begin{array}{c}\text { Sample } \\
\text { ID } \\
(\#) \\
\end{array}$ & $\begin{array}{c}\text { MD Ring } \\
\text { Crush } \\
\text { (lbf/in) }\end{array}$ & $\begin{array}{l}95 \% \\
\text { C.I. }\end{array}$ & $\begin{array}{c}\text { CD R. C. } \\
\text { Index } \\
\left(\mathrm{N}^{*} \mathrm{~m} / \mathrm{g}\right) \\
\end{array}$ & $\begin{array}{l}95 \% \\
\text { C.I. }\end{array}$ & $\begin{array}{c}\text { MD R. C. } \\
\text { Index } \\
\left(N^{*} m / g\right) \\
\end{array}$ & $\begin{array}{l}95 \% \\
\text { C.I. }\end{array}$ & $\begin{array}{c}\mathrm{CD} \\
\text { Tensile } \\
\text { (lbf/in) }\end{array}$ & $\begin{array}{l}95 \% \\
\text { C.I. }\end{array}$ & $\begin{array}{l}\text { MD } \\
\text { Tensile } \\
\text { (lbf/in) }\end{array}$ & $\begin{array}{l}95 \% \\
\text { C.I. }\end{array}$ & $\begin{array}{c}\mathrm{CD} \\
\text { Tensile } \\
\text { Index } \\
\left(\mathrm{N}^{*} \mathrm{~m} / \mathrm{g}\right) \\
\end{array}$ & $\begin{array}{l}95 \% \\
\text { C.I. }\end{array}$ & $\begin{array}{c}\text { MD } \\
\text { Tensile } \\
\text { Index } \\
\left(\mathrm{N}^{*} \mathrm{~m} / \mathrm{g}\right) \\
\end{array}$ & $\begin{array}{l}95 \% \\
\text { C.I. }\end{array}$ \\
\hline \multicolumn{15}{|l|}{$\begin{array}{c}\text { Commercial } \\
\text { Medium } \\
107811\end{array}$} \\
\hline Center & 7.33 & 0.28 & 7.87 & 0.24 & 11.26 & 0.40 & 12.63 & 0.36 & 38.01 & 1.13 & 19.04 & 0.49 & 57.02 & 1.17 \\
\hline Edge & 8.21 & 0.21 & 8.02 & 0.20 & 12.04 & 0.29 & 12.34 & 0.25 & 36.60 & 0.79 & 18.81 & 0.32 & 55.13 & 1.14 \\
\hline \multicolumn{15}{|l|}{107812} \\
\hline Center & 6.97 & 0.38 & 7.87 & 0.46 & 10.68 & 0.57 & 12.57 & 0.26 & 35.57 & 0.95 & 19.27 & 0.41 & 54.61 & 1.22 \\
\hline Edge & 7.35 & 0.21 & 7.73 & 0.30 & 11.13 & 0.28 & 12.96 & 0.17 & 36.10 & 1.00 & 19.66 & 0.23 & 54.84 & 1.44 \\
\hline \multicolumn{15}{|l|}{107813} \\
\hline Center & 7.07 & 0.20 & 7.14 & 0.43 & 10.84 & 0.26 & 11.85 & 0.32 & 34.88 & 2.51 & 18.32 & 0.48 & 53.92 & 2.97 \\
\hline Edge & 7.55 & 0.13 & 7.41 & 0.26 & 11.46 & 0.17 & 12.11 & 0.26 & 34.71 & 1.08 & 18.56 & 0.40 & 52.93 & 1.52 \\
\hline \multicolumn{15}{|l|}{107814} \\
\hline Center & 8.14 & 0.29 & 8.05 & 0.41 & 12.09 & 0.41 & 12.46 & 0.42 & 38.23 & 1.67 & 18.77 & 0.59 & 57.80 & 1.60 \\
\hline Edge & 7.88 & 0.18 & 7.73 & 0.19 & 11.82 & 0.24 & 12.41 & 0.22 & 37.51 & 0.98 & 18.80 & 0.35 & 56.59 & 1.06 \\
\hline \multicolumn{15}{|l|}{107816} \\
\hline Center & 7.63 & 0.27 & 7.55 & 0.45 & 11.55 & 0.37 & 12.61 & 0.36 & 36.36 & 1.55 & 19.28 & 0.51 & 55.44 & 1.92 \\
\hline Edge & 7.72 & 0.25 & 7.63 & 0.20 & 11.70 & 0.30 & 12.44 & 0.25 & 35.84 & 1.17 & 18.87 & 0.37 & 54.69 & 1.62 \\
\hline \multicolumn{15}{|l|}{$\begin{array}{l}\text { Commercial } \\
\text { Liner }\end{array}$} \\
\hline Center & 14.52 & 0.65 & 12.30 & 0.54 & 17.03 & 0.68 & 27.01 & 1.40 & 75.94 & 3.36 & 33.03 & 1.64 & 93.23 & 3.95 \\
\hline Edge & 14.13 & 0.43 & 11.68 & 0.39 & 16.86 & 0.36 & 26.98 & 0.58 & 75.44 & 3.08 & 32.56 & 0.80 & 90.38 & 2.95 \\
\hline 225121 & & & & & & & & & & & & & & \\
\hline Center & 14.41 & 0.50 & 12.38 & 0.55 & 16.80 & 0.57 & 24.81 & 1.40 & 73.20 & 2.87 & 29.13 & 1.77 & 85.37 & 4.32 \\
\hline Edge & 14.35 & 0.29 & 12.13 & 0.49 & 16.83 & 0.33 & 26.60 & 0.75 & 77.34 & 1.62 & 31.72 & 0.95 & 90.66 & 2.12 \\
\hline
\end{tabular}


Table E3 cont. Average Physical Property Data by Lane.

\begin{tabular}{|c|c|c|c|c|c|c|c|c|c|c|c|c|c|c|c|c|}
\hline Test Methods & T494 & & & & & & & & IPST & & IPST & & T809 & & & \\
\hline $\begin{array}{c}\text { Sample } \\
\text { ID } \\
(\#)\end{array}$ & $\begin{array}{c}\text { CD } \\
\text { Young's } \\
\text { Modulus } \\
(\text { lbf/in^2) }\end{array}$ & $\begin{array}{c}95 \% \\
\text { C.I. }\end{array}$ & $\begin{array}{c}\text { MD } \\
\text { Young's } \\
\text { Modulus } \\
\text { (lbf/in^2) }\end{array}$ & $\begin{array}{c}95 \% \\
\text { C.I. }\end{array}$ & $\begin{array}{c}\text { CD } \\
\text { Young's } \\
\text { Modulus } \\
\left(\mathrm{MN} / \mathrm{m}^{\wedge} 2\right)\end{array}$ & $\begin{array}{c}95 \% \\
\text { C.I. }\end{array}$ & $\begin{array}{c}\text { MD } \\
\text { Young's } \\
\text { Modulus } \\
\left(\mathrm{MN} / \mathrm{m}^{\wedge} 2\right)\end{array}$ & $\begin{array}{c}95 \% \\
\text { C.I. }\end{array}$ & $\begin{array}{c}\text { OD Basis } \\
\text { Weight } \\
\left(\mathrm{g} / \mathrm{m}^{\wedge} 2\right)\end{array}$ & $\begin{array}{c}95 \% \\
\text { C.I. }\end{array}$ & $\begin{array}{c}\text { OD } \\
\text { Density } \\
\left(\mathrm{g} / \mathrm{cm}^{\wedge} 3\right)\end{array}$ & $\begin{array}{l}95 \% \\
\text { C.I. }\end{array}$ & $\begin{array}{c}\text { Concora } \\
\text { (lbf) }\end{array}$ & $\begin{array}{l}95 \% \\
\text { C.I. }\end{array}$ & $\begin{array}{l}\text { Concora } \\
\text { (N) }\end{array}$ & $\begin{array}{l}95 \% \\
\text { C.I. }\end{array}$ \\
\hline $\begin{array}{l}11926 \\
\text { Front } \\
\text { Center } \\
\text { Back }\end{array}$ & $\begin{array}{l}22602 \\
20356 \\
21819\end{array}$ & $\begin{array}{l}481 \\
418 \\
806\end{array}$ & $\begin{array}{l}57384 \\
57241 \\
54920\end{array}$ & $\begin{array}{l}2057 \\
1948 \\
1172\end{array}$ & $\begin{array}{l}155.8 \\
140.3 \\
150.4\end{array}$ & $\begin{array}{l}3.3 \\
2.9 \\
5.6\end{array}$ & $\begin{array}{l}395.6 \\
394.6 \\
378.6\end{array}$ & $\begin{array}{c}14.2 \\
13.4 \\
8.1\end{array}$ & $\begin{array}{l}151.69 \\
148.03 \\
151.20\end{array}$ & $\begin{array}{l}0.71 \\
0.51 \\
0.63\end{array}$ & $\begin{array}{l}0.702 \\
0.682 \\
0.700\end{array}$ & $\begin{array}{l}0.018 \\
0.013 \\
0.021\end{array}$ & & & & \\
\hline $\begin{array}{c}11927 \\
\text { Front } \\
\text { Center } \\
\text { Back }\end{array}$ & $\begin{array}{l}21474 \\
20171 \\
21347\end{array}$ & $\begin{array}{l}385 \\
669 \\
808\end{array}$ & $\begin{array}{l}56306 \\
57194 \\
53035\end{array}$ & $\begin{array}{c}1780 \\
935 \\
1217\end{array}$ & $\begin{array}{l}148.0 \\
139.1 \\
147.2\end{array}$ & $\begin{array}{l}2.7 \\
4.6 \\
5.6\end{array}$ & $\begin{array}{l}388.2 \\
394.3 \\
365.6\end{array}$ & $\begin{array}{c}12.3 \\
6.4 \\
8.4\end{array}$ & $\begin{array}{l}149.29 \\
149.66 \\
149.21\end{array}$ & $\begin{array}{l}0.76 \\
0.44 \\
0.59\end{array}$ & $\begin{array}{l}0.732 \\
0.726 \\
0.725\end{array}$ & $\begin{array}{l}0.010 \\
0.009 \\
0.012\end{array}$ & & & & \\
\hline $\begin{array}{l}11928 \\
\text { Front } \\
\text { Center } \\
\text { Back }\end{array}$ & $\begin{array}{l}21905 \\
19738 \\
21246\end{array}$ & $\begin{array}{l}555 \\
493 \\
605\end{array}$ & $\begin{array}{l}57128 \\
57001 \\
54431\end{array}$ & $\begin{array}{c}2456 \\
1075 \\
820\end{array}$ & $\begin{array}{l}151.0 \\
136.1 \\
146.5\end{array}$ & $\begin{array}{l}3.8 \\
3.4 \\
4.2\end{array}$ & $\begin{array}{l}393.9 \\
393.0 \\
375.3\end{array}$ & $\begin{array}{c}16.9 \\
7.4 \\
5.7\end{array}$ & $\begin{array}{l}150.82 \\
150.94 \\
150.81\end{array}$ & $\begin{array}{l}0.68 \\
0.51 \\
0.65\end{array}$ & $\begin{array}{l}0.715 \\
0.720 \\
0.708\end{array}$ & $\begin{array}{l}0.016 \\
0.008 \\
0.016\end{array}$ & & & & \\
\hline $\begin{array}{l}11930 \\
\text { Front } \\
\text { Center } \\
\text { Back }\end{array}$ & $\begin{array}{l}22043 \\
20489 \\
21726\end{array}$ & $\begin{array}{l}760 \\
612 \\
459\end{array}$ & $\begin{array}{l}55948 \\
50291 \\
53774\end{array}$ & $\begin{array}{l}1087 \\
9610 \\
1800\end{array}$ & $\begin{array}{l}152.0 \\
141.3 \\
149.8\end{array}$ & $\begin{array}{l}5.2 \\
4.2 \\
3.2\end{array}$ & $\begin{array}{l}385.7 \\
346.7 \\
370.7\end{array}$ & $\begin{array}{c}7.5 \\
66.3 \\
12.4\end{array}$ & $\begin{array}{l}152.77 \\
152.37 \\
152.53\end{array}$ & $\begin{array}{l}0.75 \\
0.46 \\
0.73\end{array}$ & $\begin{array}{l}0.728 \\
0.686 \\
0.686\end{array}$ & $\begin{array}{l}0.015 \\
0.020 \\
0.020\end{array}$ & & & & \\
\hline $\begin{array}{l}11931 \\
\text { Front } \\
\text { Center } \\
\text { Back }\end{array}$ & $\begin{array}{l}21169 \\
19308 \\
20908\end{array}$ & $\begin{array}{l}911 \\
414 \\
497\end{array}$ & $\begin{array}{l}54476 \\
54621 \\
53512\end{array}$ & $\begin{array}{l}2266 \\
1813 \\
2255\end{array}$ & $\begin{array}{l}145.9 \\
133.1 \\
144.1\end{array}$ & $\begin{array}{l}6.3 \\
2.9 \\
3.4\end{array}$ & $\begin{array}{l}375.6 \\
376.6 \\
368.9\end{array}$ & $\begin{array}{l}15.6 \\
12.5 \\
15.5\end{array}$ & $\begin{array}{l}148.39 \\
149.13 \\
149.03\end{array}$ & $\begin{array}{l}0.57 \\
0.50 \\
0.79\end{array}$ & $\begin{array}{l}0.706 \\
0.678 \\
0.684\end{array}$ & $\begin{array}{l}0.020 \\
0.015 \\
0.018\end{array}$ & & & & \\
\hline $\begin{array}{l}11932 \\
\text { Front } \\
\text { Center } \\
\text { Back }\end{array}$ & $\begin{array}{l}20364 \\
19989 \\
20710\end{array}$ & $\begin{array}{c}1200 \\
268 \\
544\end{array}$ & $\begin{array}{l}54598 \\
55070 \\
51956\end{array}$ & $\begin{array}{l}2184 \\
1461 \\
1875\end{array}$ & $\begin{array}{l}140.4 \\
137.8 \\
142.8\end{array}$ & $\begin{array}{l}8.3 \\
1.8 \\
3.8\end{array}$ & $\begin{array}{l}376.4 \\
379.7 \\
358.2\end{array}$ & $\begin{array}{l}15.1 \\
10.1 \\
12.9\end{array}$ & $\begin{array}{l}147.68 \\
147.75 \\
147.81\end{array}$ & $\begin{array}{l}0.63 \\
0.55 \\
0.92\end{array}$ & $\begin{array}{l}0.671 \\
0.694 \\
0.667\end{array}$ & $\begin{array}{l}0.029 \\
0.030 \\
0.021\end{array}$ & & & & \\
\hline $\begin{array}{l}11933 \\
\text { Front } \\
\text { Center } \\
\text { Back }\end{array}$ & $\begin{array}{l}20229 \\
21234\end{array}$ & $\begin{array}{l}407 \\
812\end{array}$ & $\begin{array}{l}56758 \\
55725\end{array}$ & $\begin{array}{c}971 \\
1305\end{array}$ & $\begin{array}{l}139.5 \\
146.4\end{array}$ & $\begin{array}{l}2.8 \\
5.6\end{array}$ & $\begin{array}{l}391.3 \\
384.2\end{array}$ & $\begin{array}{l}6.7 \\
9.0\end{array}$ & $\begin{array}{l}147.76 \\
146.75 \\
147.67\end{array}$ & $\begin{array}{l}0.60 \\
0.56 \\
0.75\end{array}$ & $\begin{array}{l}0.692 \\
0.679 \\
0.686\end{array}$ & $\begin{array}{l}0.024 \\
0.018 \\
0.020\end{array}$ & & & & \\
\hline $\begin{array}{c}11934 \\
\text { Front } \\
\text { Center } \\
\text { Back }\end{array}$ & $\begin{array}{l}21585 \\
20007 \\
18066\end{array}$ & $\begin{array}{c}634 \\
626 \\
1464\end{array}$ & $\begin{array}{l}55785 \\
54425 \\
53884\end{array}$ & $\begin{array}{l}1834 \\
1713 \\
2271\end{array}$ & $\begin{array}{l}148.8 \\
137.9 \\
124.6\end{array}$ & $\begin{array}{c}4.4 \\
4.3 \\
10.1\end{array}$ & $\begin{array}{l}384.6 \\
375.2 \\
371.5\end{array}$ & $\begin{array}{l}12.6 \\
11.8 \\
15.7\end{array}$ & $\begin{array}{l}151.35 \\
150.05 \\
150.83\end{array}$ & $\begin{array}{l}0.73 \\
0.55 \\
0.78\end{array}$ & $\begin{array}{l}0.686 \\
0.657 \\
0.679\end{array}$ & $\begin{array}{l}0.022 \\
0.016 \\
0.019\end{array}$ & & & & \\
\hline
\end{tabular}


Table E3 cont. Average Physical Property Data by Lane.

\begin{tabular}{|c|c|c|c|c|c|c|c|c|c|c|c|c|c|c|c|c|}
\hline Test Methods & T494 & & & & & & & & IPST & & IPST & & T809 & & & \\
\hline $\begin{array}{c}\text { Sample } \\
\text { ID } \\
(\#)\end{array}$ & $\begin{array}{c}\mathrm{CD} \\
\text { Young's } \\
\text { Modulus } \\
\text { (lbf/in^2) }\end{array}$ & $\begin{array}{l}95 \% \\
\text { C.I. }\end{array}$ & $\begin{array}{c}\text { MD } \\
\text { Young's } \\
\text { Modulas } \\
\text { (lbffin^2) }\end{array}$ & $\begin{array}{c}95 \% \\
\text { C.I. }\end{array}$ & $\begin{array}{c}\mathrm{CD} \\
\text { Young's } \\
\text { Modulus } \\
\left(\mathrm{MN} / \mathrm{m}^{\wedge} 2\right)\end{array}$ & $\begin{array}{l}95 \% \\
\text { C.I. }\end{array}$ & $\begin{array}{c}\text { MD } \\
\text { Young's } \\
\text { Modulus } \\
\left(\mathrm{MN} / \mathrm{m}^{\wedge} 2\right)\end{array}$ & $\begin{array}{c}95 \% \\
\text { C.I. }\end{array}$ & $\begin{array}{l}\text { OD Basis } \\
\text { Weight } \\
\left(\mathrm{g} / \mathrm{m}^{\wedge} 2\right)\end{array}$ & $\begin{array}{l}95 \% \\
\text { C.I. }\end{array}$ & $\begin{array}{c}\text { OD } \\
\text { Density } \\
\left(\mathrm{g} / \mathrm{cm}^{\wedge} 3\right)\end{array}$ & $\begin{array}{l}95 \% \\
\text { C.I. }\end{array}$ & $\begin{array}{c}\text { Concora } \\
\text { (lbf) }\end{array}$ & $\begin{array}{c}95 \% \\
\text { C.I. } \\
\end{array}$ & $\begin{array}{l}\text { Concora } \\
(\mathrm{N})\end{array}$ & $\begin{array}{c}95 \% \\
\text { C.I. } \\
\end{array}$ \\
\hline $\begin{array}{l}11935 \\
\text { Front } \\
\text { Center } \\
\text { Back }\end{array}$ & $\begin{array}{l}21469 \\
19901 \\
20772\end{array}$ & $\begin{array}{l}413 \\
313 \\
321\end{array}$ & $\begin{array}{l}51977 \\
53006 \\
50376\end{array}$ & $\begin{array}{c}907 \\
1075 \\
1253\end{array}$ & $\begin{array}{l}148.0 \\
137.2 \\
143.2\end{array}$ & $\begin{array}{l}2.8 \\
2.2 \\
2.2\end{array}$ & $\begin{array}{l}358.3 \\
365.4 \\
347.3\end{array}$ & $\begin{array}{l}6.3 \\
7.4 \\
8.6\end{array}$ & $\begin{array}{l}151.43 \\
152.13 \\
152.37\end{array}$ & $\begin{array}{l}0.69 \\
0.48 \\
0.65\end{array}$ & $\begin{array}{l}0.720 \\
0.688 \\
0.678\end{array}$ & $\begin{array}{l}0.016 \\
0.015 \\
0.015\end{array}$ & & & & \\
\hline $\begin{array}{l}11936 \\
\text { Front } \\
\text { Center } \\
\text { Back }\end{array}$ & $\begin{array}{l}21453 \\
19720 \\
20729\end{array}$ & $\begin{array}{l}392 \\
347 \\
434\end{array}$ & $\begin{array}{l}51957 \\
53156 \\
52115\end{array}$ & $\begin{array}{c}1440 \\
889 \\
838\end{array}$ & $\begin{array}{l}147.9 \\
136.0 \\
142.9\end{array}$ & $\begin{array}{l}2.7 \\
2.4 \\
3.0\end{array}$ & $\begin{array}{l}358.2 \\
366.5 \\
359.3\end{array}$ & $\begin{array}{l}9.9 \\
6.1 \\
5.8\end{array}$ & $\begin{array}{l}150.20 \\
149.29 \\
150.76\end{array}$ & $\begin{array}{l}0.67 \\
0.48 \\
0.70\end{array}$ & $\begin{array}{l}0.710 \\
0.700 \\
0.696\end{array}$ & $\begin{array}{l}0.023 \\
0.011 \\
0.019\end{array}$ & & & & \\
\hline $\begin{array}{l}11937 \\
\text { Front } \\
\text { Center } \\
\text { Back } \\
\end{array}$ & & & & & & & & & & & & & & & & \\
\hline $\begin{array}{l}11938 \\
\text { Front } \\
\text { Center } \\
\text { Back }\end{array}$ & & & & & & & & & & & & & & & & \\
\hline $\begin{array}{l}11939 \\
\text { Front } \\
\text { Center } \\
\text { Back } \\
\end{array}$ & & & & & & & & & & & & & & & & \\
\hline $\begin{array}{l}11940 \\
\text { Front } \\
\text { Center } \\
\text { Back }\end{array}$ & & & & & & & & & & & & & & & & \\
\hline $\begin{array}{l}11941 \\
\text { Front } \\
\text { Center } \\
\text { Back }\end{array}$ & & & & & & & & & . & & & & & & & \\
\hline $\begin{array}{l}11942 \\
\text { Front } \\
\text { Center } \\
\text { Back }\end{array}$ & $\begin{array}{l}19517 \\
20645\end{array}$ & $\begin{array}{l}500 \\
361\end{array}$ & $\begin{array}{l}54311 \\
51827\end{array}$ & $\begin{array}{l}1997 \\
1844\end{array}$ & $\begin{array}{l}134.6 \\
142.3\end{array}$ & $\begin{array}{l}3.4 \\
2.5\end{array}$ & $\begin{array}{l}374.4 \\
357.3\end{array}$ & $\begin{array}{l}13.8 \\
12.7\end{array}$ & $\begin{array}{l}153.53 \\
151.56 \\
151.15\end{array}$ & $\begin{array}{l}0.79 \\
0.55 \\
0.82\end{array}$ & $\begin{array}{l}0.637 \\
0.617 \\
0.621\end{array}$ & $\begin{array}{l}0.013 \\
0.011 \\
0.012 \\
\end{array}$ & & & & \\
\hline
\end{tabular}


Table E3 cont. Average Physical Property Data by Lane.

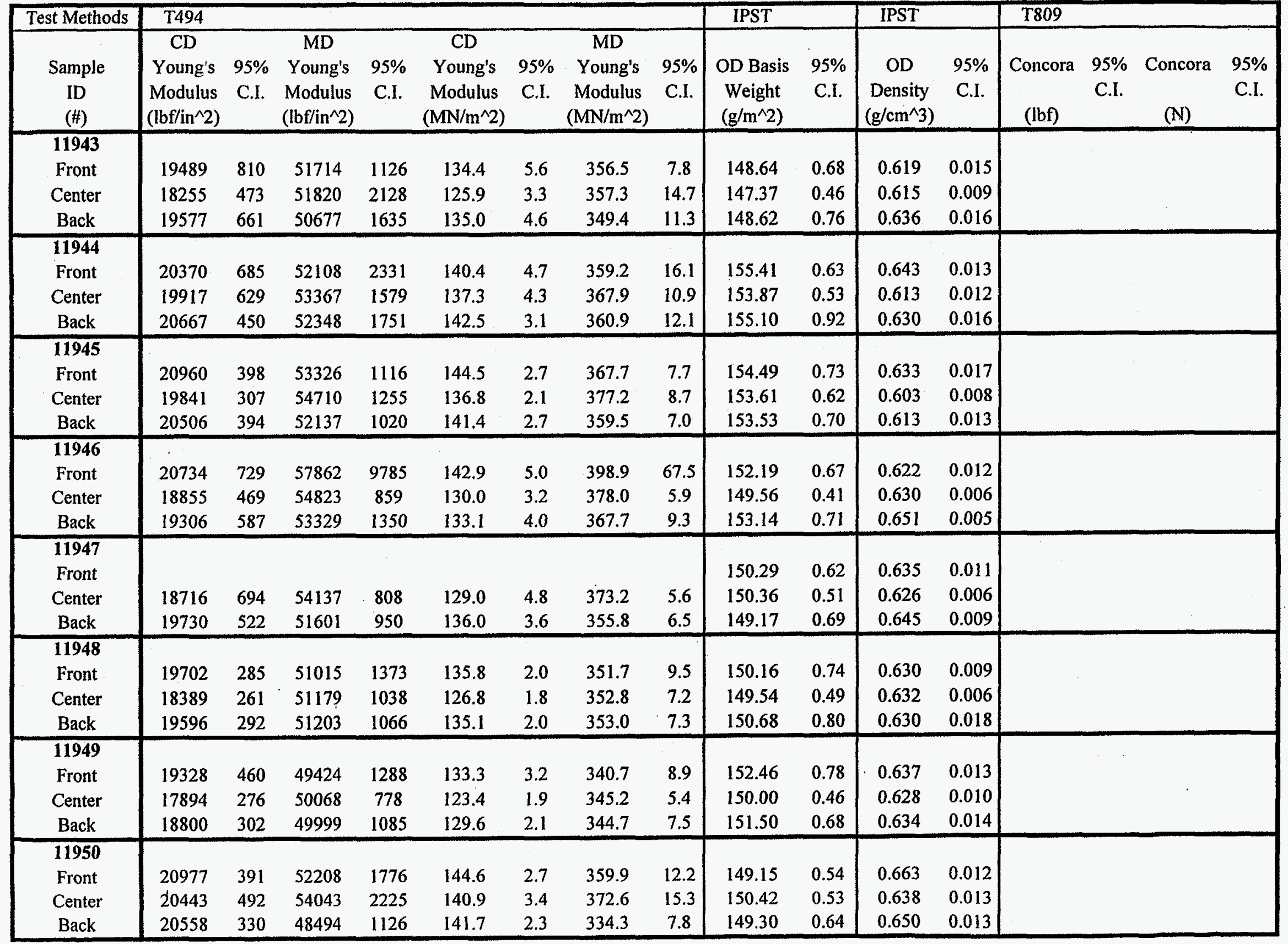


Table E3 cont. Average Physical Property Data by Lane.

\begin{tabular}{|c|c|c|c|c|c|c|c|c|c|c|c|c|c|c|c|c|}
\hline Test Methods & T494 & & & & & & & & IPST & & IPST & & T809 & & & \\
\hline $\begin{array}{l}\text { Sample } \\
\text { ID } \\
\text { (\#) }\end{array}$ & $\begin{array}{c}\text { CD } \\
\text { Young's } \\
\text { Modulus } \\
\text { (lbf/in' 2) }\end{array}$ & $\begin{array}{c}95 \% \\
\text { C.I. }\end{array}$ & $\begin{array}{c}\text { MD } \\
\text { Young's } \\
\text { Modulus } \\
\left(\mathrm{lbf} / \mathrm{in}^{\wedge} 2\right)\end{array}$ & $\begin{array}{c}95 \% \\
\text { C.I. }\end{array}$ & $\begin{array}{c}\mathrm{CD} \\
\text { Young's } \\
\text { Modulus } \\
\left(\mathrm{MN} / \mathrm{m}^{\wedge} 2\right)\end{array}$ & $\begin{array}{c}95 \% \\
\text { C.I. }\end{array}$ & $\begin{array}{c}\text { MD } \\
\text { Young's } \\
\text { Modulus } \\
\left(\mathrm{MN} / \mathrm{m}^{\wedge} 2\right)\end{array}$ & $\begin{array}{c}95 \% \\
\text { C.I. }\end{array}$ & $\begin{array}{l}\text { OD Basis } \\
\text { Weight } \\
\left(\mathrm{g} / \mathrm{m}^{\wedge} 2\right)\end{array}$ & $\begin{array}{l}95 \% \\
\text { C.I. }\end{array}$ & $\begin{array}{c}\text { OD } \\
\text { Density } \\
\left(\mathrm{g} / \mathrm{cm}^{\wedge} 3\right)\end{array}$ & $\begin{array}{l}95 \% \\
\text { C.I. }\end{array}$ & $\begin{array}{c}\text { Concora } \\
\text { (lbf })\end{array}$ & $\begin{array}{l}95 \% \\
\text { C.I. }\end{array}$ & $\begin{array}{l}\text { Concora } \\
\text { (N) }\end{array}$ & $\begin{array}{l}95 \% \\
\text { C.I. }\end{array}$ \\
\hline $\begin{array}{c}11951 \\
\text { Front } \\
\text { Center } \\
\text { Back }\end{array}$ & $\begin{array}{l}19973 \\
17928 \\
20035 \\
\end{array}$ & $\begin{array}{l}791 \\
659 \\
543 \\
\end{array}$ & $\begin{array}{l}53967 \\
55372 \\
48910 \\
\end{array}$ & $\begin{array}{l}1591 \\
1592 \\
1917\end{array}$ & $\begin{array}{l}137.7 \\
123.6 \\
138.1\end{array}$ & $\begin{array}{l}5.5 \\
4.5 \\
3.7\end{array}$ & $\begin{array}{l}372.1 \\
381.8 \\
337.2\end{array}$ & $\begin{array}{l}11.0 \\
11.0 \\
13.2\end{array}$ & $\begin{array}{l}149.72 \\
149.42 \\
149.43\end{array}$ & $\begin{array}{l}0.53 \\
0.46 \\
0.52\end{array}$ & $\begin{array}{l}0.684 \\
0.663 \\
0.688\end{array}$ & $\begin{array}{l}0.014 \\
0.016 \\
0.012\end{array}$ & & & & \\
\hline $\begin{array}{c}11952 \\
\text { Front } \\
\text { Center } \\
\text { Back }\end{array}$ & $\begin{array}{l}21083 \\
20089 \\
20244\end{array}$ & $\begin{array}{l}288 \\
400 \\
307\end{array}$ & $\begin{array}{l}53880 \\
54969 \\
51689\end{array}$ & $\begin{array}{l}1766 \\
1923 \\
1794\end{array}$ & $\begin{array}{l}145.4 \\
138.5 \\
139.6\end{array}$ & $\begin{array}{l}2.0 \\
2.8 \\
2.1\end{array}$ & $\begin{array}{l}371.5 \\
379.0 \\
356.4\end{array}$ & $\begin{array}{l}12.2 \\
13.3 \\
12.4\end{array}$ & $\begin{array}{l}147.29 \\
147.15 \\
146.82\end{array}$ & $\begin{array}{l}0.46 \\
0.41 \\
0.39\end{array}$ & $\begin{array}{l}0.694 \\
0.680 \\
0.677\end{array}$ & $\begin{array}{l}0.014 \\
0.012 \\
0.015\end{array}$ & & & & \\
\hline $\begin{array}{c}11953 \\
\text { Front } \\
\text { Center } \\
\text { Back }\end{array}$ & $\begin{array}{l}20758 \\
19454 \\
20032 \\
\end{array}$ & $\begin{array}{l}497 \\
323 \\
247\end{array}$ & $\begin{array}{l}52874 \\
52970 \\
51845 \\
\end{array}$ & $\begin{array}{l}1333 \\
1078 \\
1455\end{array}$ & $\begin{array}{l}143.1 \\
134.1 \\
138.1\end{array}$ & $\begin{array}{l}3.4 \\
2.2 \\
1.7\end{array}$ & $\begin{array}{l}364.5 \\
365.2 \\
357.4\end{array}$ & $\begin{array}{c}9.2 \\
7.4 \\
10.0\end{array}$ & $\begin{array}{l}149.30 \\
149.55 \\
148.39\end{array}$ & $\begin{array}{l}0.52 \\
0.53 \\
0.61 \\
\end{array}$ & $\begin{array}{l}0.695 \\
0.679 \\
0.679 \\
\end{array}$ & $\begin{array}{l}0.013 \\
0.014 \\
0.015\end{array}$ & & & & \\
\hline $\begin{array}{l}11954 \\
\text { Front } \\
\text { Center } \\
\text { Back } \\
\end{array}$ & $\begin{array}{l}21820 \\
19867 \\
20317 \\
\end{array}$ & $\begin{array}{l}371 \\
374 \\
367 \\
\end{array}$ & $\begin{array}{l}51894 \\
54368 \\
50945 \\
\end{array}$ & $\begin{array}{l}8283 \\
1356 \\
1558 \\
\end{array}$ & $\begin{array}{l}150.4 \\
137.0 \\
140.1\end{array}$ & $\begin{array}{l}2.6 \\
2.6 \\
2.5\end{array}$ & $\begin{array}{l}357.8 \\
374.8 \\
351.2 \\
\end{array}$ & $\begin{array}{c}57.1 \\
9.4 \\
10.7 \\
\end{array}$ & $\begin{array}{l}151.64 \\
151.76 \\
151.44 \\
\end{array}$ & $\begin{array}{l}0.49 \\
0.40 \\
0.53 \\
\end{array}$ & $\begin{array}{l}0.692 \\
0.687 \\
0.679 \\
\end{array}$ & $\begin{array}{l}0.016 \\
0.013 \\
0.014 \\
\end{array}$ & & & & \\
\hline $\begin{array}{l}11955 \\
\text { Front } \\
\text { Center } \\
\text { Back }\end{array}$ & $\begin{array}{l}20698 \\
19461 \\
20355\end{array}$ & $\begin{array}{l}654 \\
493 \\
532\end{array}$ & $\begin{array}{l}53975 \\
54644 \\
52396\end{array}$ & $\begin{array}{l}1648 \\
1307 \\
1455\end{array}$ & $\begin{array}{l}142.7 \\
134.2 \\
140.3\end{array}$ & $\begin{array}{l}4.5 \\
3.4 \\
3.7\end{array}$ & $\begin{array}{l}372.1 \\
376.7 \\
361.2\end{array}$ & $\begin{array}{c}11.4 \\
9.0 \\
10.0\end{array}$ & $\begin{array}{l}151.69 \\
152.38 \\
152.69\end{array}$ & $\begin{array}{l}0.59 \\
0.54 \\
0.65\end{array}$ & $\begin{array}{l}0.672 \\
0.640 \\
0.651\end{array}$ & $\begin{array}{l}0.017 \\
0.017 \\
0.015\end{array}$ & & & & \\
\hline $\begin{array}{l}11956 \\
\text { Front } \\
\text { Center } \\
\text { Back }\end{array}$ & $\begin{array}{l}19903 \\
19102 \\
19910\end{array}$ & $\begin{array}{l}930 \\
455 \\
267\end{array}$ & $\begin{array}{l}53212 \\
54504 \\
51943\end{array}$ & $\begin{array}{c}1341 \\
1089 \\
669\end{array}$ & $\begin{array}{l}137.2 \\
131.7 \\
137.3\end{array}$ & $\begin{array}{l}6.4 \\
3.1 \\
1.8\end{array}$ & $\begin{array}{l}366.9 \\
375.8 \\
358.1\end{array}$ & $\begin{array}{l}9.2 \\
7.5 \\
4.6\end{array}$ & $\begin{array}{l}151.80 \\
150.38 \\
150.33\end{array}$ & $\begin{array}{l}0.57 \\
0.57 \\
0.64\end{array}$ & $\begin{array}{l}0.690 \\
0.658 \\
0.660\end{array}$ & $\begin{array}{l}0.017 \\
0.016 \\
0.016\end{array}$ & & & & \\
\hline
\end{tabular}


Table E3 cont. Average Physical Property Data by Lane.

\begin{tabular}{|c|c|c|c|c|c|c|c|c|c|c|c|c|c|c|c|c|}
\hline Test Methods & T494 & & & & & & & & IPST & & IPST & & T809 & & & \\
\hline $\begin{array}{c}\text { Sample } \\
\text { ID } \\
(\#) \\
\end{array}$ & \begin{tabular}{|c|} 
CD \\
Young's \\
Modulus \\
(lbf/in^2) \\
\end{tabular} & $\begin{array}{l}95 \% \\
\text { C.I. } \\
\end{array}$ & $\begin{array}{c}\text { MD } \\
\text { Young's } \\
\text { Modulus } \\
\text { (lbf/in^2) }\end{array}$ & $\begin{array}{c}95 \% \\
\text { C.I. }\end{array}$ & $\begin{array}{c}\mathrm{CD} \\
\text { Young's } \\
\text { Modulus } \\
\left(\mathrm{MN} / \mathrm{m}^{\wedge} 2\right) \\
\end{array}$ & $\begin{array}{l}95 \% \\
\text { C.I. }\end{array}$ & $\begin{array}{c}\text { MD } \\
\text { Young's } \\
\text { Modulus } \\
\left(\mathrm{MN} / \mathrm{m}^{\wedge} 2\right) \\
\end{array}$ & $\begin{array}{c}95 \% \\
\text { C.I. }\end{array}$ & $\begin{array}{c}\text { OD Basis } \\
\text { Weight } \\
\left(\mathrm{g} / \mathrm{m}^{\wedge} 2\right) \\
\end{array}$ & $\begin{array}{l}95 \% \\
\text { C.I. }\end{array}$ & $\begin{array}{c}\text { OD } \\
\text { Density } \\
\left(\mathrm{g} / \mathrm{cm}^{\wedge} 3\right)\end{array}$ & $\begin{array}{l}95 \% \\
\text { C.I. }\end{array}$ & $\begin{array}{c}\text { Concora } \\
\text { (lbf) }\end{array}$ & $\begin{array}{c}95 \% \\
\text { C.I. } \\
\end{array}$ & $\begin{array}{c}\text { Concora } \\
(\mathrm{N}) \\
\end{array}$ & $\begin{array}{c}95 \% \\
\text { C.I. }\end{array}$ \\
\hline $\begin{array}{c}\text { Commer:ial } \\
\text { Medium } \\
107811 \\
\text { Center } \\
\text { Edge }\end{array}$ & $\begin{array}{l}10887 \\
10411\end{array}$ & $\begin{array}{l}22 \epsilon \\
182\end{array}$ & $\begin{array}{l}36889 \\
36201\end{array}$ & $\begin{array}{c}1075 \\
642\end{array}$ & $\begin{array}{l}75.1 \\
71.8\end{array}$ & $\begin{array}{l}1.6 \\
1.3\end{array}$ & $\begin{array}{l}254.3 \\
249.6\end{array}$ & $\begin{array}{l}7.4 \\
4.4\end{array}$ & $\begin{array}{l}115.64 \\
116.97\end{array}$ & $\begin{array}{l}0.61 \\
0.47\end{array}$ & $\begin{array}{l}0.616 \\
0.616\end{array}$ & $\begin{array}{l}0.010 \\
0.009\end{array}$ & $\begin{array}{l}49.05 \\
50.23\end{array}$ & $\begin{array}{l}2.71 \\
1.10\end{array}$ & $\begin{array}{l}218.17 \\
223.40\end{array}$ & $\begin{array}{c}12.06 \\
4.89\end{array}$ \\
\hline $\begin{array}{c}107812 \\
\text { Center } \\
\text { Edge }\end{array}$ & $\begin{array}{c}9949 \\
10563\end{array}$ & $\begin{array}{l}285 \\
199\end{array}$ & $\begin{array}{l}35298 \\
35596\end{array}$ & $\begin{array}{c}1124 \\
589\end{array}$ & $\begin{array}{l}68.6 \\
72.8\end{array}$ & $\begin{array}{l}2.0 \\
1.4\end{array}$ & $\begin{array}{l}243.4 \\
245.4\end{array}$ & $\begin{array}{l}7.8 \\
4.1\end{array}$ & $\begin{array}{l}115.07 \\
115.02\end{array}$ & $\begin{array}{l}5.56 \\
0.46\end{array}$ & $\begin{array}{l}0.636 \\
0.629\end{array}$ & $\begin{array}{l}0.033 \\
0.008\end{array}$ & $\begin{array}{l}49.25 \\
48.45\end{array}$ & $\begin{array}{l}1.86 \\
1.13\end{array}$ & $\begin{array}{l}219.06 \\
215.51\end{array}$ & $\begin{array}{l}8.29 \\
5.03\end{array}$ \\
\hline $\begin{array}{l}107813 \\
\text { Center } \\
\text { Edge }\end{array}$ & $\begin{array}{l}9704 \\
9728\end{array}$ & $\begin{array}{l}359 \\
247\end{array}$ & $\begin{array}{l}33890 \\
33210\end{array}$ & $\begin{array}{c}1864 \\
726\end{array}$ & $\begin{array}{l}66.9 \\
67.1\end{array}$ & $\begin{array}{l}2.5 \\
1.7\end{array}$ & $\begin{array}{l}233.6 \\
229.0\end{array}$ & $\begin{array}{c}12.9 \\
5.0\end{array}$ & $\begin{array}{l}112.91 \\
114.67\end{array}$ & $\begin{array}{l}0.69 \\
0.43\end{array}$ & $\begin{array}{l}0.626 \\
0.637\end{array}$ & $\begin{array}{l}0.014 \\
0.009\end{array}$ & $\begin{array}{r}47.85 \\
50.18\end{array}$ & $\begin{array}{l}3.01 \\
1.32\end{array}$ & $\begin{array}{l}212.84 \\
223.18 \\
\end{array}$ & $\begin{array}{c}13.40 \\
5.87\end{array}$ \\
\hline $\begin{array}{c}\mathbf{1 0 7 8 1 4} \\
\text { Center } \\
\text { Edge }\end{array}$ & $\begin{array}{l}10328 \\
10081\end{array}$ & $\begin{array}{l}238 \\
219\end{array}$ & $\begin{array}{l}37180 \\
36322\end{array}$ & $\begin{array}{l}1692 \\
1075\end{array}$ & $\begin{array}{l}71.2 \\
69.5\end{array}$ & $\begin{array}{l}1.6 \\
1.5\end{array}$ & $\begin{array}{l}256.3 \\
250.4\end{array}$ & $\begin{array}{c}11.7 \\
7.4\end{array}$ & $\begin{array}{l}116.77 \\
116.15\end{array}$ & $\begin{array}{l}0.86 \\
0.53\end{array}$ & $\begin{array}{l}0.618 \\
0.611\end{array}$ & $\begin{array}{l}0.012 \\
0.009\end{array}$ & $\begin{array}{l}51.15 \\
46.88\end{array}$ & $\begin{array}{l}1.93 \\
1.62\end{array}$ & $\begin{array}{l}227.52 \\
208.50\end{array}$ & $\begin{array}{l}8.59 \\
7.19\end{array}$ \\
\hline $\begin{array}{c}\mathbf{1 0 7 8 1 6} \\
\text { Center } \\
\text { Edge }\end{array}$ & $\begin{array}{l}10525 \\
10186 \\
\end{array}$ & $\begin{array}{l}253 \\
499 \\
\end{array}$ & $\begin{array}{l}35841 \\
36688 \\
\end{array}$ & $\begin{array}{c}2795 \\
660 \\
\end{array}$ & $\begin{array}{l}72.6 \\
70.2 \\
\end{array}$ & $\begin{array}{l}1.7 \\
3.4 \\
\end{array}$ & $\begin{array}{l}247.1 \\
252.9 \\
\end{array}$ & $\begin{array}{c}19.3 \\
4.6\end{array}$ & $\begin{array}{l}114.30 \\
115.09 \\
\end{array}$ & $\begin{array}{l}0.79 \\
0.44 \\
\end{array}$ & $\begin{array}{l}0.624 \\
0.618 \\
\end{array}$ & $\begin{array}{l}0.011 \\
0.008 \\
\end{array}$ & $\begin{array}{l}49.05 \\
52.18 \\
\end{array}$ & $\begin{array}{l}2.08 \\
1.76 \\
\end{array}$ & $\begin{array}{l}218.17 \\
232.07 \\
\end{array}$ & $\begin{array}{l}9.23 \\
7.82 \\
\end{array}$ \\
\hline $\begin{array}{c}\text { Commercial } \\
\text { Liner } \\
225111 \\
\text { Center } \\
\text { Edge }\end{array}$ & $\begin{array}{l}18752 \\
18957\end{array}$ & $\begin{array}{l}749 \\
401\end{array}$ & $\begin{array}{l}58356 \\
59941\end{array}$ & $\begin{array}{r}1699 \\
2034\end{array}$ & $\begin{array}{l}129.3 \\
130.7\end{array}$ & $\begin{array}{l}5.2 \\
2.8\end{array}$ & $\begin{array}{l}402.3 \\
413.3\end{array}$ & $\begin{array}{l}11.7 \\
14.0\end{array}$ & $\begin{array}{l}146.24 \\
146.46\end{array}$ & $\begin{array}{l}1.11 \\
0.79\end{array}$ & $\begin{array}{l}0.699 \\
0.702\end{array}$ & $\begin{array}{l}0.020 \\
0.007\end{array}$ & & & & \\
\hline $\begin{array}{c}225121 \\
\text { Center } \\
\text { Edge }\end{array}$ & $\begin{array}{l}16664 \\
18878\end{array}$ & $\begin{array}{c}1033 \\
876\end{array}$ & $\begin{array}{l}54236 \\
53573\end{array}$ & $\begin{array}{l}2255 \\
1261\end{array}$ & $\begin{array}{l}114.9 \\
130.1\end{array}$ & $\begin{array}{l}7.1 \\
6.0\end{array}$ & $\begin{array}{l}373.9 \\
369.4\end{array}$ & $\begin{array}{c}15.5 \\
8.7\end{array}$ & $\begin{array}{l}149.67 \\
145.88\end{array}$ & $\begin{array}{c}1.05 \\
14.07\end{array}$ & $\begin{array}{l}0.699 \\
0.697\end{array}$ & $\begin{array}{l}0.012 \\
0.069\end{array}$ & & & & \\
\hline
\end{tabular}


Table E4. Combined Board Test Data.

\begin{tabular}{|c|c|c|c|c|c|c|c|c|c|c|c|c|c|}
\hline Test Methods & 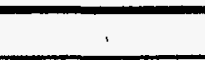 & & & & & T839 & & & & & & & \\
\hline $\begin{array}{c}\text { Case } \\
\text { Number } \\
(\#)\end{array}$ & $\begin{array}{l}\text { Corrugator } \\
\text { Group Order } \\
\text { Code }\end{array}$ & $\begin{array}{l}\text { Press } \\
\text { Temp } \\
\left({ }^{\circ} \mathrm{C}\right)\end{array}$ & $\begin{array}{l}\text { Calendar } \\
\text { Load } \\
(\mathrm{KN} / \mathrm{m})\end{array}$ & $\begin{array}{l}\text { Inner } \\
\text { Liner } \\
\text { ID } \\
(\#)\end{array}$ & $\begin{array}{c}\text { Outer } \\
\text { Liner } \\
\text { ID } \\
(\#)\end{array}$ & $\begin{array}{l}\text { Center } \\
\text { ECT } \\
\text { (lbf/in) }\end{array}$ & $\begin{array}{c}95 \% \\
\text { C.I. }\end{array}$ & $\begin{array}{l}\text { Center } \\
\text { ECT } \\
(\mathrm{kN} / \mathrm{m})\end{array}$ & $\begin{array}{c}95 \% \\
\text { C.I. }\end{array}$ & $\begin{array}{c}\text { Edge } \\
\text { ECT } \\
\text { (lbf/in) }\end{array}$ & $\begin{array}{c}95 \% \\
\text { C.I. }\end{array}$ & $\begin{array}{c}\text { Edge } \\
\text { ECT } \\
(\mathrm{kN} / \mathrm{m})\end{array}$ & $\begin{array}{c}95 \% \\
\text { C.I. }\end{array}$ \\
\hline Case 1 & & Commercial & Commercial & 225121 & 225111 & & & & & & & & \\
\hline $\begin{array}{l}\text { Group 1-10 } \\
\text { Groun } 11-20\end{array}$ & 1 & & & & & $\begin{array}{l}29.50 \\
30.09\end{array}$ & $\begin{array}{l}1.11 \\
1.59\end{array}$ & $\begin{array}{l}5.17 \\
5.27\end{array}$ & $\begin{array}{l}0.19 \\
0.28\end{array}$ & $\begin{array}{l}28.99 \\
28.62\end{array}$ & $\begin{array}{l}0.73 \\
0.98\end{array}$ & $\begin{array}{l}5.08 \\
5.01\end{array}$ & $\begin{array}{l}0.13 \\
0.17\end{array}$ \\
\hline Group 21-30 & 3 & & & & & 29.71 & 1.24 & 5.20 & 0.22 & 29.89 & 1.06 & 5.23 & 0.19 \\
\hline Group 31-40 & 4 & & & & & 30.64 & 2.91 & 5.37 & 0.51 & 31.44 & 2.33 & 5.51 & 0.41 \\
\hline Average & & & & & & 29.98 & 0.81 & 5.25 & 0.14 & 29.73 & 0.70 & 5.21 & 0.12 \\
\hline Case 2A & & 246 & 0 & 11930 & 11926 & & & & & & & & \\
\hline Group 1-10 & 5 & & & & & 26.48 & 2.19 & 4.64 & 0.38 & 30.50 & 1.11 & 5.34 & 0.19 \\
\hline Group 11-20 & 6 & & & & & 27.91 & 1.55 & 4.89 & 0.27 & 30.78 & 1.08 & 5.39 & 0.19 \\
\hline Group 21-30 & 7 & & & & & 26.46 & 0.73 & 4.63 & 0.13 & 29.23 & 1.42 & 5.12 & 0.25 \\
\hline Group 31-40 & 8 & & & & & 30.96 & 1.64 & 5.42 & 0.29 & 30.51 & 1.24 & 5.34 & 0.22 \\
\hline Average & ? & & & & & 27.95 & 0.91 & 4.89 & 0.16 & 30.25 & 0.56 & 5.30 & 0.10 \\
\hline Case 2B & & 246 & 0 & 11931 & 11932 & & & & & & & & \\
\hline Group 1-10 & 9 & & & & & 28.82 & 1.13 & 5.05 & 0.20 & 28.92 & 1.34 & 5.06 & 0.23 \\
\hline Group 11-20 & 10 & & & & & 28.66 & 1.31 & 5.02 & 0.23 & 30.16 & 1.03 & 5.28 & 0.18 \\
\hline Group 21-30 & 11 & & & & & 26.44 & 2.53 & 4.63 & 0.44 & 29.39 & 1.18 & 5.15 & 0.21 \\
\hline Group 31-40 & 12 & & & & & 31.26 & 1.34 & 5.47 & 0.24 & 30.51 & 1.44 & 5.34 & 0.25 \\
\hline Average & & & & & & 28.79 & 0.91 & 5.04 & 0.16 & 29.74 & 0.58 & 5.21 & 0.10 \\
\hline Case 3 & & wp & 35 & 11949 & 11948 & & & & & & & & \\
\hline Group 1-10 & 13 & & & & & 26.51 & 1.12 & 4.64 & 0.20 & 25.64 & 1.13 & 4.49 & 0.20 \\
\hline Group 11-20 & 14 & & & & & 28.20 & 0.41 & 4.94 & 0.07 & 28.08 & 0.66 & 4.92 & 0.12 \\
\hline Group 21-30 & 15 & & & & & 25.42 & 1.61 & 4.45 & 0.28 & 26.72 & 1.04 & 4.68 & 0.18 \\
\hline Group 31-40 & 16 & & & & & 27.97 & 1.75 & 4.90 & 0.31 & 23.61 & 0.99 & 4.13 & 0.17 \\
\hline Average & & & & & & 27.02 & 0.68 & 4.73 & 0.12 & 26.01 & 0.67 & 4.55 & $\overline{0.12}$ \\
\hline Case 4 & & 260 & 0 & 11935 & 11936 & & & & & & & & \\
\hline Group 1-10 & 17 & & & & & 28.19 & 1.60 & 4.94 & 0.28 & 31.17 & 0.99 & 5.46 & 0.17 \\
\hline Group 11-20 & 18 & & & & & 30.47 & 0.86 & 5.33 & 0.15 & 31.88 & 0.85 & 5.58 & 0.15 \\
\hline Group 21-30 & 19 & & & & & 28.64 & 0.92 & 5.02 & 0.16 & 30.03 & 1.48 & 5.26 & 0.26 \\
\hline Group 31-40 & 20 & & & & & 23.94 & 1.63 & 4.19 & 0.29 & 27.43 & 2.30 & 4.80 & 0.40 \\
\hline Average & & & & & & 27.81 & 0.96 & 4.87 & 0.17 & 30.13 & 0.85 & 5.28 & 0.15 \\
\hline
\end{tabular}


Table E4 cont. Combined Board Test Data.

\begin{tabular}{|c|c|c|c|c|c|c|c|c|c|c|c|c|c|}
\hline Test Methods & & & & & & T839 & & & & & & & \\
\hline $\begin{array}{c}\text { Case } \\
\text { Number } \\
\text { (\#) }\end{array}$ & $\begin{array}{l}\text { Corrugator } \\
\text { Group Order } \\
\text { Code }\end{array}$ & $\begin{array}{l}\text { Press } \\
\text { Temp } \\
\left({ }^{\circ} \mathrm{C}\right)\end{array}$ & $\begin{array}{c}\text { Calendar } \\
\text { Load } \\
(\mathrm{KN} / \mathrm{m})\end{array}$ & $\begin{array}{c}\text { Inner } \\
\text { Liner } \\
\text { ID } \\
(\#)\end{array}$ & $\begin{array}{c}\text { Outer } \\
\text { Liner } \\
\text { ID } \\
(\#)\end{array}$ & $\begin{array}{l}\text { Center } \\
\text { ECT } \\
\text { (lbf/in) }\end{array}$ & $\begin{array}{c}95 \% \\
\text { C.I. }\end{array}$ & $\begin{array}{l}\text { Center } \\
\text { ECT } \\
(\mathrm{kN} / \mathrm{m})\end{array}$ & $\begin{array}{c}95 \% \\
\text { C.I. }\end{array}$ & $\begin{array}{c}\text { Edge } \\
\text { ECT } \\
\text { (lbf/in) }\end{array}$ & $\begin{array}{c}95 \% \\
\text { C.I. }\end{array}$ & $\begin{array}{c}\text { Edge } \\
\mathrm{ECT} \\
(\mathrm{kN} / \mathrm{m})\end{array}$ & $\begin{array}{c}95 \% \\
\text { C.I. }\end{array}$ \\
\hline Case 5 & & wp & 0 & $\overline{11944}$ & $\overline{11945}$ & & & & & & & & \\
\hline Group 1-10 & 21 & & & & & 26.04 & 1.21 & 4.56 & 0.21 & 27.85 & 0.72 & 4.88 & 0.13 \\
\hline Group 11-20 & 22 & & & & & 28.62 & 1.15 & 5.01 & 0.20 & 27.70 & 1.18 & 4.85 & 0.21 \\
\hline Group 21-30 & 23 & & & & & 25.71 & 1.42 & 4.50 & 0.25 & 27.33 & 1.27 & 4.79 & 0.22 \\
\hline Group 31-40 & 24 & & & & & 23.81 & 2.75 & 4.17 & 0.48 & 24.69 & 1.77 & 4.32 & 0.31 \\
\hline Average & & & & & & 26.04 & 0.94 & 4.56 & 0.16 & 26.89 & 0.69 & 4.71 & 0.12 \\
\hline Case 6 & & 246 & 35 & 11927 & 11928 & & & & & & & & \\
\hline Group 1-10 & 25 & & & & & 26.33 & 1.17 & 4.61 & 0.20 & 26.36 & 0.66 & 4.62 & 0.12 \\
\hline Group 11-20 & 26 & & & & & 24.31 & 1.87 & 4.26 & 0.33 & 26.22 & 1.88 & 4.59 & 0.33 \\
\hline Group 21-30 & 27 & & & & & 26.91 & 0.97 & 4.71 & 0.17 & 27.47 & 1.07 & 4.81 & 0.19 \\
\hline Group 31-40 & 28 & & & & & 28.03 & 0.91 & 4.91 & 0.16 & 28.35 & 1.63 & 4.96 & 0.28 \\
\hline Average & & & & & & 26.39 & 0.71 & 4.62 & 0.12 & 27.10 & 0.66 & 4.75 & 0.12 \\
\hline
\end{tabular}


Table E4 cont. Combined Board Test Data.

\begin{tabular}{|c|c|c|c|c|c|c|c|c|c|c|c|c|c|c|c|c|}
\hline Test Methods & IPST & & & & T411 & & IPST & & T411 & & IPST & & $\mathrm{T} 821$ & & & \\
\hline $\begin{array}{c}\text { Case } \\
\text { Number } \\
(\#) \\
\end{array}$ & $\begin{array}{c}\text { Center } \\
\text { Glue } \\
\text { Weight } \\
\text { (gsm) } \\
\end{array}$ & $\begin{array}{l}95 \% \\
\text { C.I. }\end{array}$ & $\begin{array}{c}\text { Edge } \\
\text { Glue } \\
\text { Weight } \\
(\mathrm{gsm}) \\
\end{array}$ & $\begin{array}{l}95 \% \\
\text { C.I. }\end{array}$ & $\begin{array}{c}\text { Center } \\
\text { Combined } \\
\text { Caliper } \\
(\mathrm{mm}) \\
\end{array}$ & $\begin{array}{l}95 \% \\
\text { C.I. }\end{array}$ & $\begin{array}{l}\text { Center } \\
\text { Flute } \\
\text { Caliper } \\
(\mathrm{mm}) \\
\end{array}$ & $\begin{array}{c}95 \% \\
\text { C.I. }\end{array}$ & $\begin{array}{c}\text { Edge } \\
\text { Combined } \\
\text { Caliper } \\
(\mathrm{mm}) \\
\end{array}$ & $\begin{array}{l}95 \% \\
\text { C.I. }\end{array}$ & $\begin{array}{l}\text { Edge } \\
\text { Flute } \\
\text { Caliper } \\
(\mathrm{mm}) \\
\end{array}$ & $\begin{array}{c}95 \% \\
\text { C.I. }\end{array}$ & $\begin{array}{c}\text { Single Face } \\
\text { Center Pin } \\
\text { Adhesion } \\
(\mathrm{lbf} / \mathrm{ft})\end{array}$ & $\begin{array}{l}95 \% \\
\text { C.I. }\end{array}$ & $\begin{array}{l}\text { Single Face } \\
\text { Edge Pin } \\
\text { Adhesion } \\
(\mathrm{lbf} / \mathrm{ft})\end{array}$ & $\begin{array}{c}95 \% \\
\text { C.I. }\end{array}$ \\
\hline $\begin{array}{c}\text { Case } 1 \\
\text { Groun } 1-10\end{array}$ & & & & & & & & & & & & & & & 38.95 & \\
\hline $\begin{array}{l}\text { Group 1-10 } \\
\text { Group 11-20 }\end{array}$ & $\begin{array}{l}15.33 \\
18.31\end{array}$ & $\begin{array}{l}7.05 \\
4.47\end{array}$ & $\begin{array}{l}21.75 \\
22.91\end{array}$ & $\begin{array}{l}3.31 \\
3.29\end{array}$ & $\begin{array}{l}3.93 \\
3.96\end{array}$ & $\begin{array}{l}0.03 \\
0.03\end{array}$ & $\begin{array}{l}3.51 \\
3.53\end{array}$ & $\begin{array}{l}0.03 \\
0.03\end{array}$ & $\begin{array}{l}4.04 \\
4.04\end{array}$ & $\begin{array}{l}0.01 \\
0.02\end{array}$ & $\begin{array}{l}3.62 \\
3.62\end{array}$ & $\begin{array}{l}0.01 \\
0.02\end{array}$ & $\begin{array}{l}41.91 \\
41.42\end{array}$ & $\begin{array}{l}3.42 \\
2.89\end{array}$ & $\begin{array}{l}38.95 \\
40.90\end{array}$ & $\begin{array}{l}3.20 \\
3.21\end{array}$ \\
\hline Group 21-30 & 18.93 & 4.12 & 15.32 & 6.32 & 3.98 & 0.04 & 3.56 & 0.04 & 3.98 & 0.02 & 3.56 & 0.02 & 39.93 & 2.58 & 44.75 & 2.08 \\
\hline Group 31-40 & 19.71 & 5.14 & 22.56 & 4.26 & 4.01 & 0.03 & 3.58 & 0.03 & 4.03 & 0.02 & 3.61 & 0.02 & 39.03 & 5.15 & 35.91 & 3.47 \\
\hline Average & 18.07 & 2.35 & 20.64 & 2.17 & 3.97 & 0.02 & 3.55 & 0.02 & 4.02 & 0.01 & 3.60 & 0.01 & 40.57 & 1.61 & 40.12 & 1.67 \\
\hline Case 2A & & & & & & & & & & & & & & & & \\
\hline Group 1-10 & 20.22 & 4.91 & 23.05 & 4.95 & 3.74 & 0.09 & 3.30 & 0.09 & 3.91 & 0.02 & 3.47 & 0.02 & 41.61 & 3.75 & 42.94 & 1.45 \\
\hline Group 11-20 & 20.18 & 2.69 & 21.58 & 4.83 & 3.80 & 0.03 & 3.36 & 0.03 & 3.88 & 0.02 & 3.44 & 0.02 & 30.92 & 4.07 & 31.20 & 3.61 \\
\hline Group 21-30 & 18.63 & 3.78 & 20.46 & 5.30 & 3.74 & 0.04 & 3.30 & 0.04 & 3.77 & 0.03 & 3.33 & 0.03 & 30.96 & 2.66 & 32.66 & 3.36 \\
\hline Group 31-40 & 17.89 & 4.04 & 17.55 & 5.07 & 3.83 & 0.04 & 3.39 & 0.04 & 3.81 & 0.04 & 3.37 & 0.04 & 42.34 & 1.71 & 40.32 & 1.61 \\
\hline Average & 19.23 & 1.72 & 20.66 & 2.26 & 3.78 & 0.03 & 3.34 & 0.03 & 3.84 & 0.02 & 3.41 & 0.02 & 36.46 & 2.25 & 36.78 & 1.98 \\
\hline Case 2B & & & & & & & & & & & & & & & & \\
\hline Group 1-10 & 27.07 & 3.43 & 26.70 & 4.32 & 3.62 & 0.06 & 3.19 & 0.06 & 3.62 & 0.05 & 3.19 & 0.05 & 39.19 & 1.58 & 37.46 & 2.58 \\
\hline Group 11-20 & 24.01 & 4.02 & 26.43 & 3.75 & 3.71 & 0.02 & 3.27 & 0.03 & 3.69 & 0.03 & 3.26 & 0.03 & 39.00 & 4.15 & 36.43 & 2.22 \\
\hline Group 21-30 & 24.67 & 4.68 & 29.53 & 4.72 & 3.57 & 0.07 & 3.14 & 0.07 & 3.65 & 0.04 & 3.22 & 0.04 & 36.43 & 1.74 & 35.41 & 1.43 \\
\hline Group 31-40 & 25.48 & 2.50 & 28.29 & 3.29 & 3.85 & 0.04 & 3.41 & 0.04 & 3.70 & 0.05 & 3.27 & 0.05 & 41.29 & 2.19 & 40.32 & 1.49 \\
\hline Average & 25.31 & 1.65 & 27.74 & 1.79 & 3.69 & 0.03 & 3.25 & 0.03 & 3.67 & 0.02 & 3.24 & 0.02 & 38.97 & 1.26 & 37.40 & 1.04 \\
\hline Case 3 & & & & & & & & & & & & & & & & \\
\hline Group 1-10 & 19.92 & 3.61 & 20.49 & 2.44 & 3.67 & 0.04 & 3.19 & 0.04 & 3.75 & 0.04 & 3.27 & 0.04 & 50.32 & 2.84 & 50.89 & 1.60 \\
\hline Group 11-20 & 20.46 & 3.31 & 23.39 & 3.50 & 3.76 & 0.02 & 3.28 & 0.02 & 3.81 & 0.02 & 3.33 & 0.02 & 43.37 & 1.48 & 45.46 & 2.02 \\
\hline Group 21-30 & 19.80 & 2.68 & 21.92 & 3.09 & 3.64 & 0.05 & 3.16 & 0.05 & 3.71 & 0.04 & 3.23 & 0.04 & 37.65 & 1.90 & 37.83 & 1.60 \\
\hline Group 31-40 & 26.66 & 3.00 & 21.92 & 4.01 & 3.82 & 0.06 & 3.34 & 0.06 & 3.63 & 0.08 & 3.15 & 0.08 & 48.58 & 2.48 & 47.73 & 1.57 \\
\hline Average & 21.71 & 1.65 & 21.93 & 1.46 & 3.72 & 0.03 & 3.24 & 0.02 & 3.73 & 0.03 & 3.25 & 0.02 & 44.98 & 1.87 & 45.48 & 1.72 \\
\hline Case 4 & & & & & & & & & & & & & & & & \\
\hline Group 1-10 & 29.12 & 3.70 & 31.78 & 3.96 & 3.56 & 0.11 & 3.13 & 0.11 & 3.77 & 0.02 & 3.34 & 0.02 & 33.75 & 1.74 & 30.90 & 1.99 \\
\hline Group 11-20 & 29.27 & 3.05 & 31.82 & 3.85 & 3.80 & 0.02 & 3.37 & 0.02 & 3.87 & 0.02 & 3.44 & 0.02 & 37.84 & 1.11 & 33.94 & 1.21 \\
\hline Group 21-30 & 28.73 & 2.72 & 28.68 & 5.02 & 3.71 & 0.06 & 3.27 & 0.06 & 3.84 & 0.03 & 3.41 & 0.03 & 42.18 & 5.97 & 41.22 & 4.40 \\
\hline Group 31-40 & 23.34 & 3.22 & 27.75 & 4.13 & 3.49 & 0.09 & 3.06 & 0.09 & 3.62 & 0.07 & 3.19 & 0.07 & 44.23 & 2.21 & 45.85 & 1.75 \\
\hline Average & 27.62 & 1.59 & 30.01 & 1.92 & 3.64 & 0.04 & 3.21 & 0.04 & 3.78 & 0.03 & 3.34 & 0.02 & 39.50 & 1.94 & 37.98 & 2.22 \\
\hline
\end{tabular}


Table E4 cont. Combined Board Test Data.

\begin{tabular}{|c|c|c|c|c|c|c|c|c|c|c|c|c|c|c|c|c|}
\hline Test Methods & IPST & & & & T411 & & IPST & & $\mathrm{T} 411$ & & IPST & & $\mathrm{T} 821$ & & & \\
\hline $\begin{array}{c}\text { Case } \\
\text { Number } \\
\text { (\#) }\end{array}$ & $\begin{array}{l}\text { Center } \\
\text { Glue } \\
\text { Weight } \\
\text { (gsm) }\end{array}$ & $\begin{array}{l}95 \% \\
\text { C.I. }\end{array}$ & $\begin{array}{c}\text { Edge } \\
\text { Glue } \\
\text { Weight } \\
\text { (gsm) }\end{array}$ & $\begin{array}{l}95 \% \\
\text { C.I. }\end{array}$ & $\begin{array}{c}\text { Center } \\
\text { Combined } \\
\text { Caliper } \\
(\mathrm{mm})\end{array}$ & $\begin{array}{l}95 \% \\
\text { C.I. }\end{array}$ & $\begin{array}{l}\text { Center } \\
\text { Flute } \\
\text { Caliper } \\
\text { (mm) }\end{array}$ & $\begin{array}{l}95 \% \\
\text { C.I. }\end{array}$ & $\begin{array}{c}\text { Edge } \\
\text { Combined } \\
\text { Caliper } \\
(\mathrm{mm})\end{array}$ & $\begin{array}{l}95 \% \\
\text { C.I. }\end{array}$ & $\begin{array}{c}\text { Edge } \\
\text { Flute } \\
\text { Caliper } \\
(\mathrm{mm})\end{array}$ & $\begin{array}{l}95 \% \\
\text { C.I. }\end{array}$ & $\begin{array}{l}\text { Single Face } \\
\text { Center Pin } \\
\text { Adhesion } \\
\text { (lbf/ft) }\end{array}$ & $\begin{array}{l}95 \% \\
\text { C.I. }\end{array}$ & $\begin{array}{l}\text { Single Face } \\
\text { Edge Pin } \\
\text { Adhesion } \\
(\mathrm{lbf} / \mathrm{ft})\end{array}$ & $\begin{array}{l}95 \% \\
\text { C.I. }\end{array}$ \\
\hline Case 5 & & & & & & & & & & & & & & & & \\
\hline Group 1-10 & 12.01 & 5.51 & 15.68 & 4.76 & 3.68 & 0.16 & 3.17 & 0.16 & 3.91 & 0.02 & 3.42 & 0.02 & 51.50 & 1.37 & 50.66 & 2.53 \\
\hline Group 11-20 & 21.93 & 2.97 & 20.91 & 4.20 & 3.85 & 0.02 & 3.34 & 0.02 & 3.85 & 0.02 & 3.35 & 0.02 & 49.72 & 1.53 & 50.91 & 1.33 \\
\hline Group 21-30 & 16.04 & 3.19 & 13.16 & 5.25 & 3.73 & 0.08 & 3.22 & 0.08 & 3.75 & 0.04 & 3.25 & 0.04 & 47.91 & 2.10 & 49.05 & 2.86 \\
\hline Group 31-40 & 16.39 & 4.63 & 19.09 & 4.36 & 3.70 & 0.10 & 3.19 & 0.10 & 3.66 & 0.07 & 3.16 & 0.07 & 50.77 & 1.85 & 52.56 & 2.83 \\
\hline Average & 16.60 & 2.14 & 17.21 & 2.23 & 3.74 & 0.05 & 3.23 & 0.05 & 3.79 & 0.03 & 3.29 & 0.02 & 49.97 & 0.86 & 50.79 & 1.13 \\
\hline $\begin{array}{c}\text { Case } 6 \\
\text { Group } 1-10\end{array}$ & 21.57 & .60 & 20.50 & 76 & 3.65 & 0.04 & 324 & 0.04 & 3.64 & 0.04 & 3.23 & 0.04 & 47.08 & 4.26 & 45.02 & 2.61 \\
\hline Group 11-20 & 22.27 & 4.20 & 25.19 & 3.89 & 3.62 & 0.05 & 3.20 & 0.05 & 3.59 & 0.08 & 3.17 & 0.08 & 42.37 & 2.26 & 43.19 & 2.98 \\
\hline Group 21-30 & 21.65 & 3.01 & 25.96 & 4.84 & 3.62 & 0.03 & 3.20 & 0.03 & 3.59 & 0.08 & 3.18 & 0.08 & 38.86 & 2.19 & 35.00 & 2.89 \\
\hline Group 31-40 & 25.99 & 2.84 & 24.02 & 2.39 & 3.58 & 0.04 & 3.17 & 0.04 & 3.55 & 0.05 & 3.13 & 0.05 & 38.44 & 2.42 & 38.32 & 1.40 \\
\hline Average & 22.87 & 1.71 & 23.92 & 1.88 & 3.62 & 0.02 & 3.20 & 0.02 & 3.59 & 0.03 & 3.18 & 0.03 & 41.69 & 1.68 & 40.38 & 1.69 \\
\hline
\end{tabular}


Table E4 cont. Combined Board Test Data.

\begin{tabular}{|c|c|c|c|c|c|c|c|c|c|c|c|c|}
\hline Test Methods & T821 & & & & & & & & & & & \\
\hline & Double Face & & Double Face & & Single Face & & Single Face & & Double Face & & Double Face & \\
\hline Case & Center Pin & $95 \%$ & Edge Pin & $95 \%$ & Center Pin & $95 \%$ & Edge Pin & $95 \%$ & Center Pin & $95 \%$ & Edge Pin & $95 \%$ \\
\hline $\begin{array}{c}\text { Number } \\
(\#)\end{array}$ & $\begin{array}{c}\text { Adhesion } \\
(\mathrm{lbf} / \mathrm{ft})\end{array}$ & C.I. & $\begin{array}{c}\text { Adhesion } \\
(\mathrm{lbf} / \mathrm{ft})\end{array}$ & C.I. & $\begin{array}{c}\text { Adhesion } \\
(\mathrm{N} / \mathrm{m})\end{array}$ & C.I. & $\begin{array}{l}\text { Adhesion } \\
(\mathrm{N} / \mathrm{m})\end{array}$ & C.I. & $\begin{array}{c}\text { Adhesion } \\
(\mathrm{N} / \mathrm{m})\end{array}$ & C.I. & $\begin{array}{l}\text { Adhesion } \\
(\mathrm{N} / \mathrm{m})\end{array}$ & C.I. \\
\hline Case 1 & & & & & & & & & & & & \\
\hline Group 1-10 & 44.49 & 3.21 & 39.58 & 3.26 & 611.56 & 49.91 & 568.36 & 47.60 & 649.21 & 46.89 & 577.63 & 47.57 \\
\hline Group 11-20 & 41.12 & 3.33 & 38.46 & 3.73 & 604.41 & 42.23 & 596.89 & 46.86 & 600.10 & 48.64 & 561.28 & 54.42 \\
\hline Group 21-30 & 39.47 & 5.03 & 40.91 & 4.00 & 582.66 & 37.60 & 653.00 & 30.36 & 575.95 & 73.48 & 597.04 & 58.38 \\
\hline Group $31-40$ & 46.76 & 4.41 & 41.44 & 6.72 & 569.53 & 75.12 & 523.99 & 50.63 & 682.34 & 64.29 & 604.77 & 98.02 \\
\hline Average & 42.96 & 1.98 & 40.10 & 2.02 & 592.04 & 23.50 & 585.56 & 24.42 & 626.90 & 28.82 & 585.18 & 29.52 \\
\hline Case 2A & & & & & & & & & & & & \\
\hline Group 1-10 & 50.75 & 2.86 & 48.88 & 2.14 & 607.25 & 54.68 & 626.59 & 21.18 & 740.64 & 41.68 & 713.35 & 31.25 \\
\hline Group 11-20 & 44.21 & 2.72 & 43.96 & 4.51 & 451.24 & 59.35 & 455.33 & 52.74 & 645.20 & 39.73 & 641.55 & 65.83 \\
\hline Group 21-30 & 42.56 & 1.89 & 39.01 & 3.78 & 451.83 & 38.89 & 476.56 & 48.99 & 621.04 & 27.58 & 569.31 & 55.23 \\
\hline Group 31-40 & 56.14 & 1.68 & 54.13 & 1.46 & 617.83 & 25.02 & 588.43 & 23.54 & 819.30 & 24.54 & 789.97 & 21.31 \\
\hline Average & 48.41 & 2.02 & 46.50 & 2.29 & 532.04 & 32.87 & 536.73 & 28.91 & 706.54 & 29.50 & 678.54 & $\overline{33.36}$ \\
\hline Case 2B & & & & & & & & & & & & \\
\hline Group 1-10 & 50.69 & 3.41 & 48.51 & 2.22 & 571.86 & 23.08 & 546.69 & 37.70 & 739.69 & 49.71 & 707.88 & 32.41 \\
\hline Group 11-20 & 45.88 & 3.51 & 47.10 & 1.99 & 569.09 & 60.56 & 531.66 & 32.45 & 669.49 & 51.17 & 687.37 & 29.03 \\
\hline Group 21-30 & 48.59 & 3.01 & 46.40 & 1.64 & 531.58 & 25.43 & 516.70 & 20.83 & 709.04 & 43.99 & 677.08 & 23.93 \\
\hline Group 31-40 & 60.94 & 2.10 & 57.59 & 3.92 & 602.58 & 32.00 & 588.43 & 21.77 & 889.35 & 30.70 & 840.46 & 57.23 \\
\hline Average & 51.52 & 2.27 & 49.90 & 1.84 & 568.78 & 18.37 & 545.87 & 15.20 & 751.90 & 33.06 & 728.20 & 26.80 \\
\hline Case 3 & & & & & & & & & & & & \\
\hline Group 1-10 & 52.09 & 2.09 & 49.64 & 1.39 & 734.29 & 41.38 & 742.68 & 23.36 & 760.12 & 30.52 & 724.44 & 20.25 \\
\hline Group 11-20 & 49.07 & 8.61 & 49.79 & 1.79 & 632.86 & 21.53 & 663.44 & 29.50 & 716.12 & \#\#\#\# & 726.56 & 26.16 \\
\hline Group 21-30 & 46.58 & 2.99 & 43.82 & 2.68 & 549.39 & 27.70 & 552.01 & 23.37 & 679.78 & 43.69 & 639.50 & 39.11 \\
\hline Group 31-40 & 54.94 & 1.93 & 53.57 & 1.81 & 708.97 & 36.13 & 696.49 & 22.90 & 801.79 & 28.11 & 781.79 & 26.34 \\
\hline Average & 50.67 & 2.29 & 49.20 & 1.41 & 656.38 & 27.25 & 663.66 & 25.17 & 739.45 & 33.41 & 718.07 & 20.60 \\
\hline Case 4 & & & & & & & & & & & & \\
\hline Group 1-10 & 42.66 & 1.97 & 37.12 & 2.20 & 492.47 & 25.33 & 450.95 & 29.04 & 622.50 & 28.72 & 541.73 & 32.10 \\
\hline Group 11-20 & 45.02 & 1.54 & 40.44 & 2.72 & 552.23 & 16.15 & 495.32 & 17.69 & 656.94 & 22.48 & 590.18 & 39.67 \\
\hline Group 21-30 & 44.28 & 3.39 & 42.10 & 3.92 & 615.50 & 87.11 & 601.49 & 64.20 & 646.14 & 49.48 & 614.40 & 57.20 \\
\hline Group 31-40 & 51.13 & 3.81 & 49.45 & 2.85 & 645.49 & 32.23 & 669.13 & 25.55 & 746.11 & 55.60 & 721.67 & 41.60 \\
\hline Average & 45.77 & 1.60 & 42.28 & 1.95 & 576.42 & 28.37 & 554.22 & 32.39 & 667.93 & 23.39 & 616.99 & 28.39 \\
\hline
\end{tabular}


Table E4 cont. Combined Board Test Data.

\begin{tabular}{|c|c|c|c|c|c|c|c|c|c|c|c|c|}
\hline Test Methods & T821 & & & & & & & & & & & \\
\hline $\begin{array}{c}\text { Case } \\
\text { Number } \\
(\#)\end{array}$ & $\begin{array}{l}\text { Double Face } \\
\text { Center Pin } \\
\text { Adhesion } \\
\text { (lbf/ft) }\end{array}$ & $\begin{array}{l}95 \% \\
\text { C.I. }\end{array}$ & $\begin{array}{l}\text { Double Face } \\
\text { Edge Pin } \\
\text { Adhesion } \\
\text { (lbf/ft) }\end{array}$ & $\begin{array}{l}95 \% \\
\text { C.I. }\end{array}$ & $\begin{array}{l}\text { Single Face } \\
\text { Center Pin } \\
\text { Adhesion } \\
(\mathrm{N} / \mathrm{m})\end{array}$ & $\begin{array}{l}95 \% \\
\text { C.I. }\end{array}$ & $\begin{array}{l}\text { Single Face } \\
\text { Edge Pin } \\
\text { Adhesion } \\
(\mathrm{N} / \mathrm{m})\end{array}$ & $\begin{array}{l}95 \% \\
\text { C.I. }\end{array}$ & $\begin{array}{c}\text { Double Face } \\
\text { Center Pin } \\
\text { Adhesion } \\
(\mathrm{N} / \mathrm{m})\end{array}$ & $\begin{array}{l}95 \% \\
\text { C.I. }\end{array}$ & $\begin{array}{c}\text { Double Face } \\
\text { Edge Pin } \\
\text { Adhesion } \\
(\mathrm{N} / \mathrm{m})\end{array}$ & $\begin{array}{c}95 \% \\
\text { C.I. }\end{array}$ \\
\hline $\begin{array}{c}\text { Case 5 } \\
\text { Group 1-10 }\end{array}$ & 46.40 & 2.21 & 40.82 & 2.46 & 751.51 & 20.03 & 739.33 & 36.96 & 677.08 & 32.18 & 595.72 & 35.94 \\
\hline Group $11-20$ & 53.08 & 2.36 & 46.50 & 1.17 & 725.61 & 22.35 & 742.90 & 19.45 & 774.57 & 34.40 & 678.62 & 17.14 \\
\hline Group 21-30 & 48.08 & 2.59 & 39.94 & 5.47 & 699.19 & 30.58 & 715.83 & 41.71 & 701.67 & 37.85 & 582.88 & 79.86 \\
\hline Group $31-40$ & 48.03 & 1.89 & 48.42 & 1.69 & 740.86 & 26.94 & 767.05 & 41.28 & 700.94 & 27.61 & 706.56 & 24.65 \\
\hline Average & 48.90 & 1.27 & 43.92 & 1.80 & 729.29 & 12.60 & 741.28 & 16.55 & 713.57 & 18.55 & 640.95 & 26.22 \\
\hline $\begin{array}{c}\text { Case } 6 \\
\text { Group 1-10 }\end{array}$ & 47.55 & 2.12 & 47.42 & 2.64 & 687.01 & 62.13 & 657.02 & 38.08 & 693.87 & 30.99 & 691.97 & 38.55 \\
\hline Group $11-20$ & 54.11 & 2.50 & 52.45 & 3.10 & 618.34 & 33.04 & 630.31 & 43.49 & 789.60 & 36.47 & 765.38 & 45.27 \\
\hline Group 21-30 & 47.86 & 4.56 & 40.89 & 3.93 & 567.12 & 31.90 & 510.71 & 42.14 & 698.39 & 66.52 & 596.74 & 57.32 \\
\hline Group $31-40$ & 47.88 & 3.18 & 44.90 & 2.55 & 560.99 & 35.28 & 559.24 & 20.47 & 698.76 & 46.43 & 655.27 & 37.28 \\
\hline Average & 49.35 & 1.65 & 46.41 & 1.90 & 608.36 & 24.52 & 589.32 & 24.61 & 720.15 & 24.05 & 677.34 & 27.76 \\
\hline
\end{tabular}


Table E4 cont. Combined Board Test Data.

\begin{tabular}{|c|c|c|c|c|c|c|c|c|c|c|c|c|c|c|c|c|}
\hline Test Methods & $\mathrm{T} 825$ & & & & & & & & T836 & & & & & & & \\
\hline $\begin{array}{c}\text { Case } \\
\text { Number } \\
\text { (\#) }\end{array}$ & $\begin{array}{c}\text { Center } \\
\text { Flat } \\
\text { Crush } \\
\text { (psi) }\end{array}$ & $\begin{array}{c}95 \% \\
\text { C.I. }\end{array}$ & $\begin{array}{l}\text { Edge } \\
\text { Flat } \\
\text { Crush } \\
\text { (psi) }\end{array}$ & $\begin{array}{l}95 \% \\
\text { C.I. }\end{array}$ & $\begin{array}{l}\text { Center } \\
\text { Flat } \\
\text { Crush } \\
(\mathrm{kPa})\end{array}$ & $\begin{array}{l}95 \% \\
\text { C.I. }\end{array}$ & $\begin{array}{c}\text { Edge } \\
\text { Flat } \\
\text { Crush } \\
(\mathrm{kPa})\end{array}$ & $\begin{array}{c}95 \% \\
\text { C.I. }\end{array}$ & $\begin{array}{c}\text { MD } \\
\text { Flexural } \\
\text { Stiffness } \\
\text { (lb-in) }\end{array}$ & $\begin{array}{c}95 \% \\
\text { C.I. }\end{array}$ & $\begin{array}{c}\text { CD } \\
\text { Flexural } \\
\text { Stiffness } \\
\text { (lb-in) }\end{array}$ & $\begin{array}{c}95 \% \\
\text { C.I. }\end{array}$ & $\begin{array}{c}\text { MD } \\
\text { Flexural } \\
\text { Stiffness } \\
(\mathrm{N}-\mathrm{m})\end{array}$ & $\begin{array}{c}95 \% \\
\text { C.I. }\end{array}$ & $\begin{array}{c}\text { CD } \\
\text { Flexural } \\
\text { Stiffness } \\
(\mathrm{N}-\mathrm{m})\end{array}$ & $\begin{array}{c}\text { 95\% } \\
\text { C.I. }\end{array}$ \\
\hline Case 1 & 2120 & 145 & 2727 & 263 & 14615 & 1001 & 18801 & 1812 & 0882 & 436 & 4321 & 008 & 1116 & 040 & 488 & 011 \\
\hline Group 11-20 & 28.46 & 1.38 & 22.97 & 5.36 & 196.21 & 9.50 & 158.39 & 36.98 & 105.26 & 2.58 & 43.53 & 0.63 & 11.89 & 0.29 & 4.92 & 0.07 \\
\hline Group 21-30 & 29.63 & 3.06 & 29.48 & 1.21 & 204.30 & 21.08 & 203.28 & 8.32 & 87.85 & 17.83 & 43.69 & 0.59 & 9.93 & 2.01 & 4.94 & 0.07 \\
\hline Group 31-40 & 28.73 & 1.75 & 29.87 & 0.70 & 198.09 & 12.07 & 205.92 & 4.85 & 56.03 & 28.98 & 45.64 & 0.66 & 6.33 & 3.27 & 5.16 & 0.07 \\
\hline Average & 27.00 & 1.40 & 27.40 & 1.59 & 186.19 & 9.64 & 188.90 & 10.95 & 86.99 & 9.60 & 44.02 & 0.44 & 9.83 & 1.08 & 4.97 & 0.05 \\
\hline Case 2A & & & & & & & & & & & & & & & & \\
\hline Group 1-10 & 21.15 & 1.05 & 22.31 & 0.50 & 145.80 & 7.23 & 153.83 & 3.47 & 71.92 & 17.82 & 38.43 & 0.35 & 8.13 & 2.01 & 4.34 & 0.04 \\
\hline Group 11-20 & 24.44 & 1.14 & 24.48 & 1.40 & 168.51 & 7.85 & 168.79 & 9.66 & 88.29 & 2.55 & 38.09 & 0.52 & 9.97 & 0.29 & 4.30 & 0.06 \\
\hline Group 21-30 & 24.53 & 0.62 & 24.83 & 1.47 & 169.16 & 4.28 & 171.18 & 10.12 & 82.94 & 4.85 & 37.09 & 0.80 & 9.37 & 0.55 & 4.19 & 0.09 \\
\hline Group 31-40 & 27.18 & 0.68 & 25.60 & 1.23 & 187.42 & 4.69 & 176.53 & 8.49 & 65.01 & 17.09 & 39.44 & 0.24 & 7.35 & 1.93 & 4.46 & 0.03 \\
\hline Average & 24.33 & 0.79 & 24.31 & 0.65 & 167.72 & 5.47 & 167.58 & 4.51 & 77.04 & 6.18 & 38.27 & 0.35 & 8.70 & 0.70 & 4.32 & 0.04 \\
\hline Case 2B & & & & & & & & & & & & & & & & \\
\hline Group 1-10 & $22.4 \%$ & 1.31 & 22.08 & 0.75 & 154.62 & 9.01 & 152.26 & 5.18 & 79.22 & 2.25 & 33.33 & 0.45 & 8.95 & 0.25 & 3.77 & 0.05 \\
\hline Group 11-20 & 21.84 & 1.46 & 23.17 & 1.25 & 150.57 & 10.07 & 159.78 & 8.63 & 75.83 & 9.37 & 34.06 & 0.63 & 8.57 & 1.06 & 3.85 & 0.07 \\
\hline Group 21-30 & 21.89 & 1.19 & 23.90 & 0.85 & 150.90 & 8.21 & 164.79 & 5.84 & 79.39 & 1.34 & 33.57 & 0.64 & 8.97 & 0.15 & 3.79 & 0.07 \\
\hline Group 31-40 & 24.15 & 0.54 & 25.66 & 1.00 & 166.51 & 3.73 & 176.95 & 6.91 & 88.29 & 24.96 & 37.75 & 0.79 & 9.98 & 2.82 & 4.27 & 0.09 \\
\hline Average & 22.57 & 0.59 & 23.70 & 0.60 & 155.65 & 4.07 & 163.44 & 4.11 & 80.68 & 5.94 & 34.68 & 0.64 & 9.12 & 0.67 & 3.92 & 0.07 \\
\hline Case 3 & & & & & & & & & & & & & & & & \\
\hline Group 1-10 & 18.89 & 0.93 & 19.17 & 1.21 & 130.25 & 6.44 & 132.17 & 8.33 & 76.25 & 1.95 & 30.73 & 0.53 & 8.61 & 0.22 & 3.47 & 0.06 \\
\hline Group 11-20 & 22.02 & 1.09 & 18.93 & 0.73 & 151.83 & 7.49 & 130.52 & 5.02 & 76.64 & 1.05 & 30.92 & 0.53 & 8.66 & 0.12 & 3.49 & 0.06 \\
\hline Group 21-30 & 22.02 & 1.66 & 22.09 & 1.80 & 151.81 & 11.43 & 152.29 & 12.40 & 75.09 & 1.61 & 30.10 & 0.62 & 8.48 & 0.18 & 3.40 & 0.07 \\
\hline Group 31-40 & 28.68 & 0.71 & 29.36 & 1.47 & 197.75 & 4.93 & 202.44 & 10.12 & 87.10 & 3.82 & 34.47 & 1.79 & 9.84 & 0.43 & 3.89 & 0.20 \\
\hline Average & 22.90 & 1.26 & 22.39 & 1.48 & 157.91 & 8.68 & 154.35 & 10.24 & 78.77 & 1.87 & 31.56 & 0.71 & 8.90 & 0.21 & 3.57 & 0.08 \\
\hline Case 4 & & & & & & & & & & & & & & & & \\
\hline Gr & 24.92 & & .10 & & & 1 & 19 & .97 & $\begin{array}{l}85.28 \\
80.90\end{array}$ & 52 & $\begin{array}{l}36.39 \\
2705\end{array}$ & 93 & 9.63 & .40 & 4.11 & $\begin{array}{l}0.11 \\
0.10\end{array}$ \\
\hline Group 21-30 & $\begin{array}{l}20.20 \\
23.29\end{array}$ & 1.00 & 25.98 & $\begin{array}{l}1.37 \\
1.76\end{array}$ & $\begin{array}{l}194.84 \\
160.57\end{array}$ & 11.43 & $\begin{array}{l}1 / 9.13 \\
16625\end{array}$ & $\begin{array}{c}9.43 \\
1212\end{array}$ & $\begin{array}{l}09.90 \\
82.44\end{array}$ & $\begin{array}{l}1.07 \\
1478\end{array}$ & 38.24 & $\begin{array}{l}0.90 \\
109\end{array}$ & $\begin{array}{l}10.10 \\
931\end{array}$ & $\begin{array}{l}0.19 \\
1.67\end{array}$ & $\begin{array}{l}4.29 \\
4.32\end{array}$ & $\begin{array}{l}0.10 \\
0.12\end{array}$ \\
\hline Group 31-40 & 27.48 & 1.27 & 27.46 & 2.08 & 189.50 & 8.76 & 189.30 & 14.34 & 90.04 & 2.02 & 39.76 & 0.88 & 10.17 & 0.23 & 4.49 & 0.10 \\
\hline Average & 25.99 & 0.83 & 25.81 & 0.89 & 179.19 & 5.73 & 177.97 & 6.14 & 86.91 & 3.47 & 38.08 & 0.56 & 9.82 & 0.39 & 4.30 & 0.06 \\
\hline
\end{tabular}


Table E4 cont. Combined Board Test Data.

\begin{tabular}{|c|c|c|c|c|c|c|c|c|c|c|c|c|c|c|c|c|}
\hline Test Methods & T825 & & & & & & & & T836 & & & & & & & \\
\hline $\begin{array}{c}\text { Case } \\
\text { Number } \\
\text { (\#) }\end{array}$ & $\begin{array}{l}\text { Center } \\
\text { Flat } \\
\text { Crush } \\
\text { (psi) }\end{array}$ & $\begin{array}{l}95 \% \\
\text { C.I. }\end{array}$ & $\begin{array}{l}\text { Edge } \\
\text { Flat } \\
\text { Crush } \\
\text { (psi) }\end{array}$ & $\begin{array}{l}95 \% \\
\text { C.I. }\end{array}$ & $\begin{array}{c}\text { Center } \\
\text { Flat } \\
\text { Crush } \\
(\mathrm{kPa})\end{array}$ & $\begin{array}{l}95 \% \\
\text { C.I. }\end{array}$ & $\begin{array}{c}\text { Edge } \\
\text { Flat } \\
\text { Crush } \\
\text { (kPa) }\end{array}$ & $\begin{array}{c}95 \% \\
\text { C.1. }\end{array}$ & $\begin{array}{c}\text { MD } \\
\text { Flexural } \\
\text { Stiffness } \\
\text { (lb-in) }\end{array}$ & $\begin{array}{c}95 \% \\
\text { C.I. }\end{array}$ & $\begin{array}{c}\text { CD } \\
\text { Flexural } \\
\text { Stiffness } \\
(\text { lb-in) }\end{array}$ & $\begin{array}{l}95 \% \\
\text { C.I. }\end{array}$ & $\begin{array}{c}\text { MD } \\
\text { Flexural } \\
\text { Stiffness } \\
\text { (N-m) }\end{array}$ & $\begin{array}{c}95 \% \\
\text { C.1. }\end{array}$ & $\begin{array}{c}\text { CD } \\
\text { Flexural } \\
\text { Stiffness } \\
(\mathrm{N}-\mathrm{m})\end{array}$ & $\begin{array}{c}95 \% \\
\text { C.I. }\end{array}$ \\
\hline $\begin{array}{c}\text { Case } 5 \\
\text { Group 1-10 }\end{array}$ & 24.19 & 1.93 & 23.39 & 0.89 & 166.82 & 13.34 & 161.26 & 6.13 & 74.34 & 24.37 & 33.19 & 2.28 & 8.40 & 2.75 & 3.75 & 0.26 \\
\hline Group 11-20 & 23.19 & 0.96 & 25.95 & 0.67 & 159.92 & 6.64 & 178.91 & 4.63 & 76.22 & 14.05 & 33.66 & 0.77 & 8.61 & 1.59 & 3.80 & 0.09 \\
\hline Group 21-30 & 23.28 & 0.87 & 25.39 & 1.16 & 160.52 & 5.97 & 175.06 & 8.01 & 72.56 & 7.87 & 32.09 & 1.04 & 8.20 & 0.89 & 3.62 & 0.12 \\
\hline Group 31-40 & 26.67 & 1.28 & 28.40 & 0.54 & 183.91 & 8.80 & 195.84 & 3.73 & 89.02 & 3.09 & 35.54 & 1.31 & 10.06 & 0.35 & 4.02 & 0.15 \\
\hline Average & 24.34 & 0.73 & 25.78 & 0.68 & 167.79 & 5.03 & 177.77 & 4.72 & 78.03 & 6.65 & 33.62 & 0.75 & 8.82 & 0.75 & 3.80 & 0.08 \\
\hline $\begin{array}{c}\text { Case } 6 \\
\text { Group 1-10 }\end{array}$ & 18.87 & 0.94 & 20.22 & 0.52 & 130.07 & 6.49 & 139.42 & 3.56 & 74.73 & 11.33 & 34.57 & 1.13 & 8.44 & 1.28 & 3.91 & 0.13 \\
\hline Group 11-20 & 27.23 & 1.67 & 26.62 & 3.14 & 187.78 & 11.48 & 183.52 & 21.65 & 102.49 & 25.25 & 38.07 & 0.75 & 11.58 & 2.85 & 4.30 & 0.08 \\
\hline Group 21-30 & 20.77 & 1.22 & 21.50 & 1.42 & 143.24 & 8.40 & 148.21 & 9.79 & 68.19 & 9.26 & 34.81 & 1.01 & 7.70 & 1.05 & 3.93 & 0.11 \\
\hline Group 31-40 & 20.35 & 0.77 & 22.34 & 0.90 & 140.31 & 5.32 & 154.05 & 6.22 & 66.10 & 13.72 & 35.59 & 1.20 & 7.47 & 1.55 & 4.02 & 0.14 \\
\hline Average & 21.81 & 1.16 & 22.67 & 1.10 & 150.35 & 8.00 & 156.30 & 7.56 & 77.88 & 8.38 & 35.76 & 0.63 & 8.80 & 0.95 & 4.04 & 0.07 \\
\hline
\end{tabular}


Table E4 cont. Combined Board Test Data.

\begin{tabular}{|c|c|c|c|c|c|c|}
\hline Test Methods & T411 & & IPST & T411 & & IPST \\
\hline $\begin{array}{l}\text { Case } \\
\text { Number } \\
\text { (\#) }\end{array}$ & $\begin{array}{c}\text { MD Flex } \\
\text { Combined } \\
\text { Caliper } \\
(\mathrm{mm})\end{array}$ & $\begin{array}{l}95 \% \\
\text { C.I. }\end{array}$ & $\begin{array}{c}\text { MD Flex } \\
\text { Flute } \\
\text { Caliper } \\
(\mathrm{mm})\end{array}$ & $\begin{array}{l}\text { CD Flex } \\
\text { Combined } \\
\text { Caliper } \\
(\mathrm{mm})\end{array}$ & $\begin{array}{l}95 \% \\
\text { C.I. }\end{array}$ & $\begin{array}{l}\text { CD Flex } \\
\text { Flute } \\
\text { Caliper } \\
(\mathrm{mm})\end{array}$ \\
\hline $\begin{array}{c}\text { Case 1 } \\
\text { Group } 1-10\end{array}$ & 4.01 & 0.02 & 4.00 & 4.01 & 0.01 & 4.01 \\
\hline Group 11-20 & 4.02 & 0.01 & 4.01 & 4.01 & 0.01 & 4.00 \\
\hline Group 21-30 & 4.05 & 0.01 & 4.04 & 4.04 & 0.01 & 4.03 \\
\hline Group 31-40 & 4.07 & 0.02 & 4.07 & 4.09 & 0.01 & 4.09 \\
\hline Average & 4.04 & 0.01 & & 4.04 & 0.01 & \\
\hline$\overline{C a s e} 2 \mathrm{~A}$ & & & & & & \\
\hline Group 1-10 & 3.82 & 0.03 & 3.81 & 3.82 & 0.02 & 3.82 \\
\hline Group 11-20 & 3.84 & 0.01 & 3.83 & 3.81 & 0.02 & 3.81 \\
\hline Group 21-30 & 3.75 & 0.02 & 3.75 & 3.73 & 0.02 & 3.72 \\
\hline Group 31-40 & 3.86 & 0.01 & 3.85 & 3.87 & 0.01 & 3.87 \\
\hline Average & 3.82 & 0.01 & & 3.81 & 0.02 & \\
\hline Case 2B & & & & & & \\
\hline Group 1-10 & 3.72 & 0.02 & 3.70 & 3.66 & 0.02 & 3.65 \\
\hline Group 11-20 & 3.71 & 0.02 & 3.70 & 3.64 & 0.03 & 3.63 \\
\hline Group 21-30 & 3.67 & 0.03 & 3.66 & 3.62 & 0.05 & 3.61 \\
\hline Group 31-40 & 3.82 & 0.02 & 3.81 & 3.83 & 0.02 & 3.82 \\
\hline Average & 3.73 & 0.02 & & 3.69 & 0.02 & \\
\hline Case 3 & & & & & & \\
\hline Group 1-10 & 3.74 & 0.02 & 3.73 & 3.70 & 0.04 & 3.69 \\
\hline Group 11-20 & 3.77 & 0.01 & 3.76 & 3.71 & 0.02 & 3.71 \\
\hline Group 21-30 & 3.71 & 0.03 & 3.71 & 3.67 & 0.02 & 3.66 \\
\hline Group 31-40 & 3.87 & 0.06 & 3.86 & 3.83 & 0.08 & 3.83 \\
\hline Average & 3.77 & 0.02 & & 3.73 & 0.03 & \\
\hline Case 4 & & & & & & \\
\hline Group 1-10 & 3.75 & 0.04 & 3.74 & 3.73 & 0.04 & 3.72 \\
\hline Group 11-20 & 3.83 & 0.01 & 3.83 & 3.80 & 0.03 & 3.79 \\
\hline Group 21-30 & 3.83 & 0.03 & 3.83 & 3.80 & 0.03 & 3.79 \\
\hline Group 31-40 & 3.86 & 0.05 & 3.85 & 3.85 & 0.04 & 3.84 \\
\hline Average & 3.82 & 0.02 & & 3.79 & 0.02 & \\
\hline
\end{tabular}


Table E4 cont. Combined Board Test Data.

\begin{tabular}{|c|ccccccc|}
\hline Test Methods & T411 & \multicolumn{3}{c}{ IPST } & T411 & IPST \\
\hline $\begin{array}{c}\text { Case } \\
\text { Number } \\
\text { (\#) }\end{array}$ & $\begin{array}{c}\text { MD Flex } \\
\text { Combined } \\
\text { Caliper } \\
(\mathrm{mm})\end{array}$ & $\begin{array}{c}\text { MD Flex } \\
\text { C.I. }\end{array}$ & $\begin{array}{c}\text { CD Flex } \\
\text { Caliper } \\
(\mathrm{mm})\end{array}$ & $\begin{array}{c}\text { Combined } \\
\text { Caliper } \\
(\mathrm{mm})\end{array}$ & $\begin{array}{c}\text { CD } \\
\text { C.I. }\end{array}$ & $\begin{array}{c}\text { Flute } \\
\text { Caliper } \\
\text { (mm) }\end{array}$ \\
\hline Case 5 & & & & & & \\
Group 1-10 & 3.69 & 0.19 & 3.68 & 3.78 & 0.09 & 3.77 \\
Group 11-20 & 3.88 & 0.01 & 3.87 & 3.84 & 0.02 & 3.83 \\
Group 21-30 & 3.75 & 0.03 & 3.74 & 3.73 & 0.03 & 3.72 \\
Group 31-40 & 3.90 & 0.04 & 3.90 & 3.88 & 0.04 & 3.87 \\
\hline Average & $\mathbf{3 . 8 1}$ & $\mathbf{0 . 0 5}$ & & $\mathbf{3 . 8 1}$ & $\mathbf{0 . 0 3}$ & \\
\hline Case 6 & & & & & & \\
Group 1-10 & 3.69 & 0.04 & 3.69 & 3.68 & 0.03 & 3.67 \\
Group 11-20 & 3.78 & $\mathbf{0 . 0 6}$ & 3.77 & 3.81 & 0.02 & 3.81 \\
Group 21-30 & 3.66 & $\mathbf{0 . 0 4}$ & 3.66 & $\mathbf{3 . 6 6}$ & 0.04 & 3.66 \\
Group 31-40 & 3.66 & 0.03 & 3.66 & $\mathbf{3 . 6 7}$ & $\mathbf{0 . 0 2}$ & 3.66 \\
\hline Average & $\mathbf{3 . 7 0}$ & $\mathbf{0 . 0 2}$ & & $\mathbf{3 . 7 0}$ & $\mathbf{0 . 0 2}$ & \\
\hline
\end{tabular}


Table E5. Box Test Data.

\begin{tabular}{|c|c|c|c|c|c|c|c|c|c|}
\hline Test Methods & & & & & & T813 & & & \\
\hline $\begin{array}{c}\text { Case } \\
\text { Number } \\
(\#)\end{array}$ & $\begin{array}{l}\text { Corrugator } \\
\text { Group Order } \\
\text { Code }\end{array}$ & $\begin{array}{l}\text { Press } \\
\text { Temp } \\
\left({ }^{\circ} \mathrm{C}\right)\end{array}$ & $\begin{array}{c}\text { Calendar } \\
\text { Load } \\
(\mathrm{KN} / \mathrm{m})\end{array}$ & $\begin{array}{l}\text { Inner } \\
\text { Liner } \\
\text { ID } \\
(\#)\end{array}$ & $\begin{array}{c}\text { Outer } \\
\text { Liner } \\
\text { ID } \\
(\#)\end{array}$ & $\begin{array}{c}\text { Manufactures } \\
\text { Glue } \\
\text { Joint } \\
\text { (lbf/in) } \\
\end{array}$ & $\begin{array}{l}95 \% \\
\text { C.I. }\end{array}$ & $\begin{array}{c}\text { Manufactures } \\
\text { Glue } \\
\text { Joint } \\
(\mathrm{kN} / \mathrm{m}) \\
\end{array}$ & $\begin{array}{l}95 \% \\
\text { C.I. }\end{array}$ \\
\hline Case 1 & & ommercial & Commercial & 225121 & 225111 & & & & \\
\hline Group 1-10 & 1 & & & & & 91.16 & 3.37 & 15.96 & 0.59 \\
\hline Group 11-20 & 2 & & & & & 92.16 & 5.34 & 16.14 & 0.94 \\
\hline Group 21-30 & 3 & & & & & 90.99 & 3.91 & 15.93 & 0.68 \\
\hline Average & & & & & & 91.44 & 2.17 & 16.01 & 0.38 \\
\hline Case 2A & & 246 & $\overline{0}$ & 11930 & 11926 & & & & \\
\hline Group 1-10 & 5 & & & & & 53.41 & 7.90 & 9.35 & 1.38 \\
\hline Group 11-20 & 6 & & & & & 47.98 & 5.83 & 8.40 & 1.02 \\
\hline Group 21-30 & 7 & & & & & 50.68 & 6.56 & 8.87 & 1.15 \\
\hline Average & & & & & & 50.69 & 3.54 & 8.88 & 0.62 \\
\hline Case 2B & & 246 & 0 & 11931 & 11932 & & & & \\
\hline Group 1-10 & 9 & & & & & 68.71 & 5.86 & 12.03 & 1.03 \\
\hline Group 11-20 & 10 & & & & & 69.42 & 3.78 & 12.16 & 0.66 \\
\hline Group 21-30 & 11 & & & & & 71.09 & 4.89 & 12.45 & 0.86 \\
\hline Average & & & & & & 69.74 & 2.51 & 12.21 & 0.44 \\
\hline Case 3 & & wp & 35 & 11949 & 11948 & & & & \\
\hline Group 1-10 & 13 & & & & & 58.82 & 7.59 & 10.30 & 1.33 \\
\hline Group 11-20 & 14 & & & & & 52.39 & 4.08 & 9.17 & 0.71 \\
\hline Group 21-30 & 15 & & & & & 58.51 & 8.18 & 10.25 & 1.43 \\
\hline Average & & & & & & 56.57 & 3.63 & 9.91 & 0.64 \\
\hline Case 4 & & 260 & 0 & 11935 & 11936 & & & & \\
\hline Group 1-10 & 17 & & & & & 59.85 & 5.47 & 10.48 & 0.96 \\
\hline Group 11-20 & 18 & & & & & 64.61 & 5.43 & 11.31 & 0.95 \\
\hline Group 21-30 & 19 & & & & & 65.08 & 5.52 & 11.40 & 0.97 \\
\hline Average & & & & & & 63.18 & 2.90 & 11.06 & 0.51 \\
\hline Case 5 & & $\overline{\text { wp }}$ & 0 & 11944 & 11945 & & & & \\
\hline Group 1-10 & 21 & & & & & 61.26 & 5.80 & 10.73 & 1.02 \\
\hline Group 11-20 & 22 & & & & & 58.44 & 2.36 & 10.23 & 0.41 \\
\hline Group 21-30 & 23 & & & & & 56.97 & 5.98 & 9.98 & 1.05 \\
\hline Average & & & & & & 58.89 & 2.61 & 10.31 & 0.46 \\
\hline
\end{tabular}


Table E5 cont. Box Test Data.

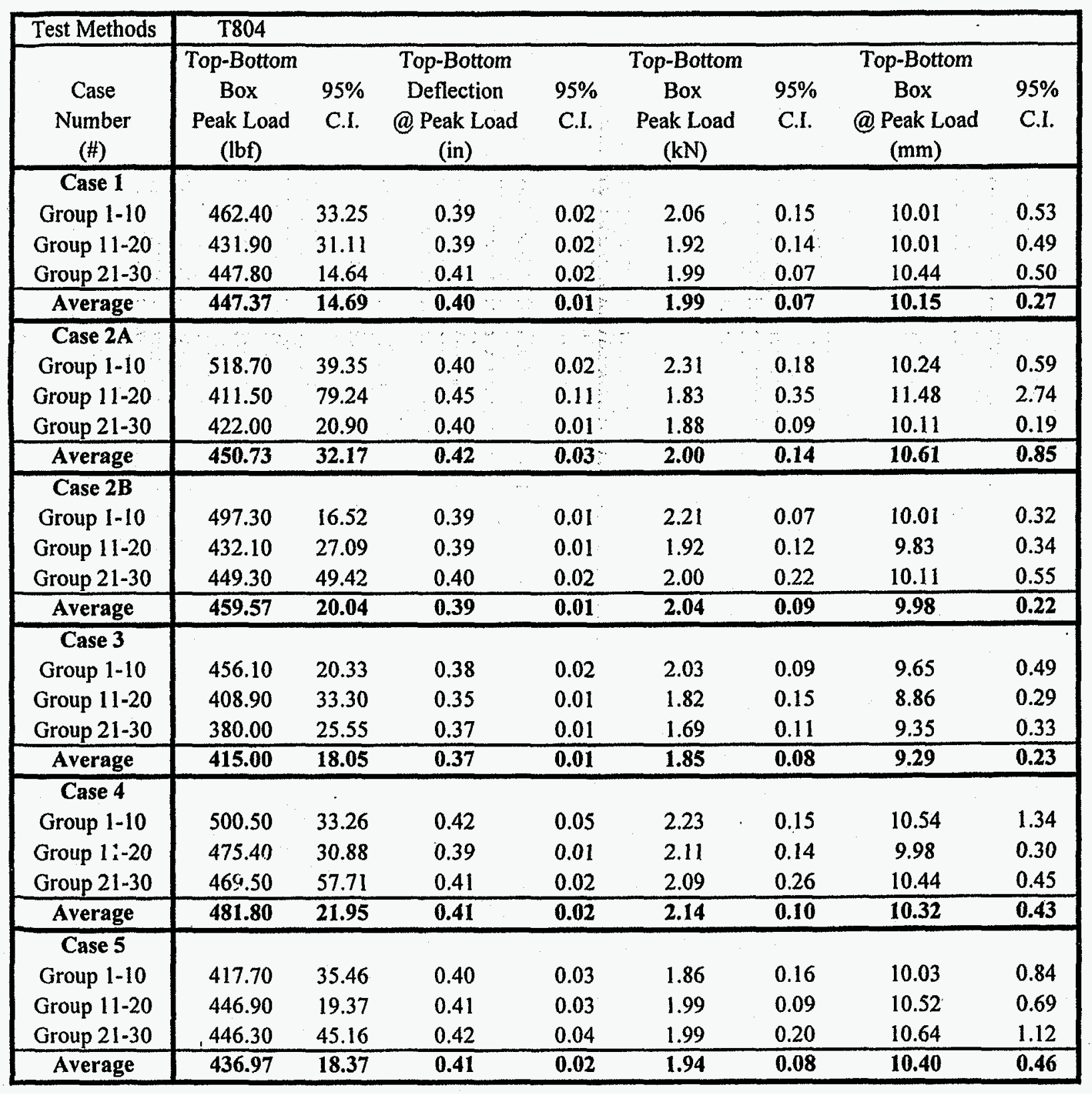


Table E5 cont. Box Test Data.

\begin{tabular}{|c|c|c|c|c|c|c|c|c|}
\hline Test Methods & T804 & & & & & & & \\
\hline $\begin{array}{c}\text { Case } \\
\text { Number } \\
(\#)\end{array}$ & $\begin{array}{l}\text { Side-Side } \\
\text { Box } \\
\text { Peak Load } \\
\text { (lbf) }\end{array}$ & $\begin{array}{l}95 \% \\
\text { C.I. }\end{array}$ & $\begin{array}{c}\text { Side-Side } \\
\text { Deflection } \\
@ \text { Peak Load } \\
\text { (in) }\end{array}$ & $\begin{array}{l}95 \% \\
\text { C.I. }\end{array}$ & $\begin{array}{l}\text { Side-Side } \\
\text { Box } \\
\text { Peak Load } \\
(\mathrm{kN})\end{array}$ & $\begin{array}{l}95 \% \\
\text { C.I. }\end{array}$ & $\begin{array}{c}\text { Side-Side } \\
\text { Box } \\
\text { @ Peak Load } \\
(\mathrm{mm})\end{array}$ & $\begin{array}{l}95 \% \\
\text { C.I. }\end{array}$ \\
\hline $\begin{array}{c}\text { Case 1 } \\
\text { Group 1-10 }\end{array}$ & 391.50 & 25.43 & 0.54 & 0.09 & 1.74 & 0.11 & 13.77 & 2.16 \\
\hline Group $11-20$ & 340.40 & 28.43 & 0.65 & 0.08 & 1.51 & 0.13 & 16.43 & 1.92 \\
\hline Group 21-30 & 360.70 & 33.94 & 0.59 & 0.07 & 1.60 & 0.15 & 14.99 & 1.77 \\
\hline Average & 364.20 & 16.85 & 0.59 & 0.04 & 1.62 & 0.07 & 15.06 & 1.07 \\
\hline Case 2A & & & & & & & & \\
\hline Group 1-10 & 347.20 & 45.95 & 0.54 & 0.17 & 1.54 . & 0.20 & 13.72 & 4.38 \\
\hline Group 11-20 & 391.80 & 10.04 & 0.54 & 0.04 & 1.74 & 0.04 & 13.69 & 1.07 \\
\hline Group 21-30 & 370.20 & 26.46 & 0.56 & 0.05 & 1.65 & 0.12 & 14.25 & 1.27 \\
\hline Average & 369.73 & 17.15 & 0.55 & 0.05 & 1.64 & 0.08 & 13.89 & 1.37 \\
\hline Case 2B & & & & & & & & \\
\hline Group 1-10 & 334.80 & 13.42 & 0.48 & 0.04 & 1.49 & 0.06 & 12.22 & 1.12 \\
\hline Group $11-20$ & 373.50 & 30.50 & 0.45 & 0.08 & 1.66 & 0.14 & 11.30 & 2.01 \\
\hline Group 21-30 & 327.20 & 28.20 & 0.44 & 0.11 & 1.46 & 0.13 & 11.13 & 2.88 \\
\hline Average & 345.17 & 14.85 & 0.45 & 0.04 & 1.54 & 0.07 & 11.55 & 1.09 \\
\hline Case 3 & & & & & & & & \\
\hline Group 1-10 & 354.70 & 27.95 & 0.47 & 0.05 & 1.58 & 0.12 & 12.04 & 1.26 \\
\hline Group 11-20 & 295.20 & 22.66 & 0.31 & 0.13 & 1.31 & 0.10 & 7.87 & 3.25 \\
\hline Group 21-30 & 322.00 & 24.84 & 0.39 & 0.11 & 1.43 & 0.11 & 9.96 & 2.91 \\
\hline Average & 323.97 & 15.72 & 0.39 & 0.06 & 1.44 & 0.07 & 9.96 & 1.47 \\
\hline Case 4 & & & & & & & & \\
\hline Group 1-10 & 432.80 & 17.08 & 0.51 & 0.03 & 1.93 & 0.08 & 13.03 & 0.80 \\
\hline Group 11-20 & 400.40 & 29.35 & 0.48 & 0.07 & 1.78 & 0.13 & 12.09 & 1.87 \\
\hline Group 21-30 & 351.10 & 26.47 & 0.51 & 0.05 & 1.56 & 0.12 & 13.03 & 1.35 \\
\hline Average & $394.7 ?$ & 17.88 & 0.50 & 0.03 & 1.76 & 0.08 & 12.72 & 0.73 \\
\hline Case 5 & & & & & & & & \\
\hline Group 1-10 & 431.10 & 27.22 & 0.46 & 0.04 & 1.92 & 0.12 & 11.66 & 0.89 \\
\hline Group 11-20 & 384.10 & 37.22 & 0.42 & 0.08 & 1.71 & 0.17 & 10.69 & 1.92 \\
\hline Group 21-30 & 312.00 & 15.16 & 0.36 & 0.08 & 1.39 & 0.07 & 9.02 & 2.07 \\
\hline Average & 375.73 & 23.35 & 0.41 & 0.04 & 1.67 & 0.10 & 10.46 & 0.96 \\
\hline
\end{tabular}


Table E5 cont. Box Test Data.

\begin{tabular}{|c|c|c|c|c|c|c|c|c|}
\hline Test Methods & T804 & & & & & & & \\
\hline $\begin{array}{c}\text { Case } \\
\text { Number } \\
(\#)\end{array}$ & $\begin{array}{l}\text { End-End } \\
\text { Box } \\
\text { Peak Load } \\
\text { (lbf) }\end{array}$ & $\begin{array}{c}95 \% \\
\text { C.I. }\end{array}$ & $\begin{array}{c}\text { End-End } \\
\text { Deflection } \\
\text { @ Peak Load } \\
\text { (in) }\end{array}$ & $\begin{array}{c}95 \% \\
\text { C.I. }\end{array}$ & $\begin{array}{l}\text { End-End } \\
\text { Box } \\
\text { Peak Load } \\
(\mathrm{kN})\end{array}$ & $\begin{array}{l}95 \% \\
\text { C.I. }\end{array}$ & $\begin{array}{c}\text { End-End } \\
\text { Box } \\
@ \text { Peak Load } \\
(\mathrm{mm}) \\
\end{array}$ & $\begin{array}{c}95 \% \\
\text { C.I. }\end{array}$ \\
\hline Case 1 & & & & & & & & \\
\hline Group 1-10 & 284.80 & 24.00 & 0.24 & 0.04 & 1.27 & 0.11 & 6.17 & 0.94 \\
\hline Group 11-20 & 291.50 & 20.29 & 0.27 & 0.04 & 1.30 & 0.09 & 6.83 & 0.92 \\
\hline Group 21-30 & 278.10 & 28.00 & 0.22 & 0.02 & 1.24 & 0.12 & 5.69 & 0.58 \\
\hline Average & 284.80 & 12.41 & 0.25 & 0.02 & 1.27 & 0.06 & 6.23 & 0.45 \\
\hline Case 2A & & & & & & & & \\
\hline Group 1-10 & 297.40 & 36.22 & 0.24 & 0.03 & 1.32 & 0.16 & 6.02 & 0.64 \\
\hline Group 11-20 & 317.40 & 18.95 & 0.24 & 0.03 & 1.41 & 0.08 & 6.07 & 0.81 \\
\hline Group 21-30 & 303.50 & 18.85 & 0.25 & 0.03 & 1.35 & 0.08 & 6.22 & 0.69 \\
\hline Average & 306.10 & 13.47 & 0.24 & 0.01 & 1.36 & 0.06 & 6.10 & 0.36 \\
\hline Case 2B & & & & & & & & \\
\hline Group 1-10 & 262.00 & 26.87 & 0.31 & 0.18 & 1.17 & 0.12 & 7.87 & 4.57 \\
\hline Group 11-20 & 267.70 & 25.73 & 0.25 & 0.05 & 1.19 & 0.11 & 6.30 & 1.18 \\
\hline Group 21-30 & 259.70 & 38.57 & 0.30 & 0.18 & 1.16 & 0.17 & 7.72 & 4.57 \\
\hline Average & 263.13 & 15.64 & 0.29 & 0.08 & 1.17 & 0.07 & 7.30 & 1.93 \\
\hline Case 3 & & & & & & & & \\
\hline Group 1-10 & 271.20 & 18.78 & 0.23 & 0.03 & 1.21 & 0.08 & 5.79 & 0.68 \\
\hline Group 1 1-20 & 288.60 & 17.78 & 0.25 & 0.03 & 1.28 & 0.08 & 6.22 & 0.65 \\
\hline Group 21-30 & 287.00 & 10.70 & 0.23 & 0.02 & 1.28 & 0.05 & 5.77 & 0.45 \\
\hline Average & 282.27 & 8.67 & 0.23 & 0.01 & 1.26 & 0.04 & 5.93 & 0.31 \\
\hline Case 4 & & & & & & & & \\
\hline Group 1-10 & 331.50 & 22.50 & 0.26 & 0.02 & 1.47 & 0.10 & 6.55 & 0.57 \\
\hline Group $11-20$ & 317.40 & 18.57 & 0.25 & 0.03 & 1.41 & 0.08 & 6.38 & 0.64 \\
\hline Group 21-30 & 303.10 & 22.83 & 0.24 & 0.03 & 1.35 & 0.10 & 6.20 & 0.78 \\
\hline Average & 317.33 & 11.64 & 0.25 & 0.01 & 1.41 & 0.05 & 6.38 & 0.34 \\
\hline Case 5 & & & & & & & & \\
\hline Group 1-10 & $282.4 \mathrm{C}$ & 21.24 & 0.26 & 0.05 & 1.26 & 0.09 & 6.50 & 1.19 \\
\hline Group 11-20 & 308.80 & 20.75 & 0.24 & 0.04 & 1.37 & 0.09 & 6.20 & 0.98 \\
\hline Group 21-30 & 303.20 & 29.11 & 0.25 & 0.04 & 1.35 & 0.13 & 6.45 & 1.08 \\
\hline Average & 298.13 & 12.84 & 0.25 & 0.02 & 1.33 & 0.06 & 6.38 & 0.55 \\
\hline
\end{tabular}

